\title{
WestVirginiaUniversity
}

THE RESEARCH REPOSITORY @ WVU

Graduate Theses, Dissertations, and Problem Reports

2008

\section{Automatic Control and Fault Diagnosis of MEMS Lateral Comb Resonators}

Afshin Izadian

West Virginia University

Follow this and additional works at: https://researchrepository.wvu.edu/etd

\section{Recommended Citation}

Izadian, Afshin, "Automatic Control and Fault Diagnosis of MEMS Lateral Comb Resonators" (2008). Graduate Theses, Dissertations, and Problem Reports. 4384.

https://researchrepository.wvu.edu/etd/4384

This Dissertation is protected by copyright and/or related rights. It has been brought to you by the The Research Repository @ WVU with permission from the rights-holder(s). You are free to use this Dissertation in any way that is permitted by the copyright and related rights legislation that applies to your use. For other uses you must obtain permission from the rights-holder(s) directly, unless additional rights are indicated by a Creative Commons license in the record and/ or on the work itself. This Dissertation has been accepted for inclusion in WVU Graduate Theses, Dissertations, and Problem Reports collection by an authorized administrator of The Research Repository @ WVU.

For more information, please contact researchrepository@mail.wvu.edu. 


\title{
Automatic Control and Fault Diagnosis of MEMS Lateral Comb Resonators
}

\author{
Afshin Izadian \\ Dissertation submitted to the \\ College of Engineering and Mineral Resources \\ at West Virginia University \\ in partial fulfillment of the requirements \\ for the degree of
}

Doctor of Philosophy

in

Electrical Engineering

Parviz Famouri, Ph.D., Chair

Lawrence A. Hornak, Ph.D.

Muhammad Choudhry, Ph.D.

Ronald Klein, Ph.D.

Marcello Napolitano, Ph.D.

Boyd Edwards, Ph.D.

Lane Department of Computer Science and Electrical Engineering

Morgantown, West Virginia 2008

Keywords: Adaptive Control, Parameter Estimation, Fault Diagnosis, MEMS, LCR 


\title{
ABSTRACT \\ Automatic Control and Fault Diagnosis of MEMS Lateral Comb Resonators
}

\begin{abstract}
Afshin Izadian
Recent advancements in microfabrication of Micro Electro Mechanical Systems have made MEMS an important part of many applications such as safety and sensor/control devices. Miniature structure of MEMS makes them very sensitive to the environmental and operating conditions. In addition, fault in the device might change the parameters and result in unwanted behavioral variations. Therefore, imperfect device structure, fault and operating point dependencies suggest for active control of MEMS.

This research is focused on two main areas of control and fault diagnosis of MEMS devices. In the control part, the application of adaptive controllers is introduced for trajectory control of the device under health and fault conditions. Fault in different forms in the structure of the device are modeled and foundry manufactured for experimental verifications. Pull-in voltage effect in the MEMS Lateral Comb Resonators are investigated and controlled by variable structure controllers. Reliability of operation is enhanced by active control of the device under fault conditions.

The second part of this research is focused on the fault diagnosis of the MEMS devices. Fault is introduced and investigated for better understanding of the system behavioral changes. Modeling of the device in different operating conditions suggests for the multiple-model adaptive estimation (MMAE) fault diagnosis technique. Application of Kalman filters in MMAE is investigated and the performance of the fault diagnosis is compared with other techniques such as self-tuning and auto self-tuning techniques. According to the varying parameters of the system, online parameter identification systems are required to monitor the parameter variations and model the system accurately. Self-tuning banks are applied and combined with MMAE to provide accurate fault diagnosis systems. Different parameter identification techniques result in different system performances. In this regard, this research investigates the application of Recursive Least Square with Forgetting Factor. Different techniques for tuning of forgetting factor value are introduced and their results are compared for better performance.

The organization of this dissertation is as follows:

Chapter I introduces the structure of the MEMS Lateral Comb Resonator; Chapter II introduces the application of control techniques and displacement feedback approach. Chapter III investigates the control approach and experimental results. In chapter IV, a new controller is introduced and designed for the MEMS trajectory controls. Chapter V is about the fault and different techniques of fault diagnosis in MEMS LCRs. Chapter 6 is the future work suggested through the current results and observations. Each chapter contains a section to summarize the concluding remarks.
\end{abstract}


To my Parents and my wife Pardis 


\section{Acknowledgement:}

I would like to thank all previous members of WVU MEMS research group, whose work has made this research possible. Especially, I would like to thank Dr. Parviz Famouri for his scientific and financial help during my research. I would like to thank Dr. Lawrence Hornak, Dr. Jeremy Dawson, Dr. Limin Wang and Ms. Deepaseri Lakshemi Konduparthi for their research and scholarly work where I have continued and made use of their techniques in my research. I would like to thank my committee members for their help and constructive guidance in preparation of this dissertation. I specially thank the mental support of my parents whom gave me what I needed to accomplish this important. Finally yet importantly, I would like to thank the support and endurance of my wife "Pardis" during my studies and hard work. 
Table of content

Chapter 1

\section{MEMS Devices: Manufacturing and Parameter Uncertainties}

1.1 Micro Electro Mechanical System ..............................................................................................

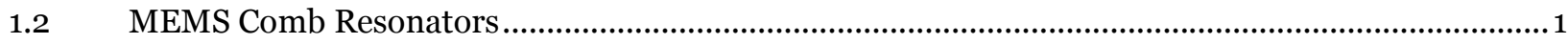

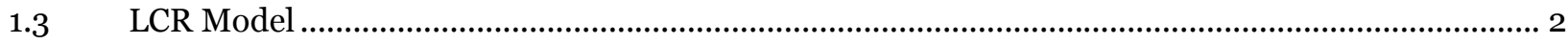

1.4 MEMS Parameter Uncertainties and Manufacturing Steps ....................................................... 6

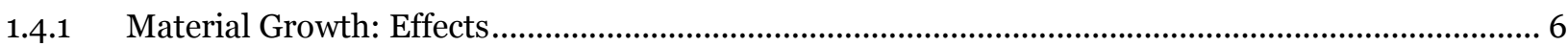

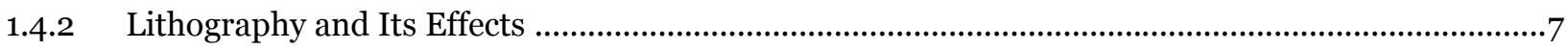

1.4.3 Effect of Etching Process on Parameters of MEMS .......................................................................... 8

1.4.4 Effect of Stress, Stress Gradient, Wearing and Stiction in Deposited Films ................................. 9

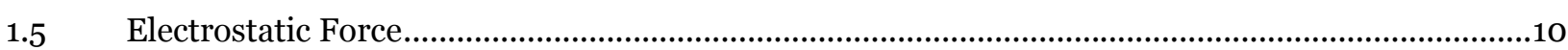

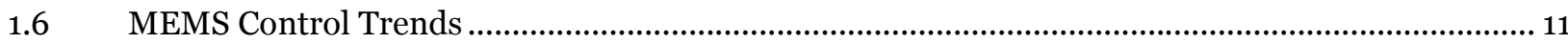

\section{Chapter 2}

\section{Control Concept and Displacement Feedback Techniques}

$2.1 \quad$ Introduction to Control................................................................................................................

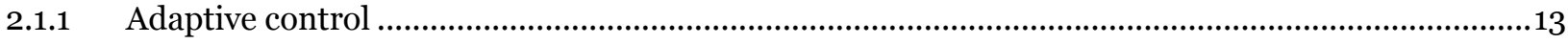

2.1.2 Model Reference Adaptive Controller..........................................................................................14

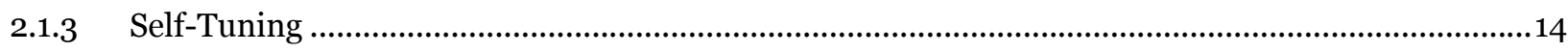

$2.2 \quad$ Displacement Feedback Techniques ....................................................................................... 15

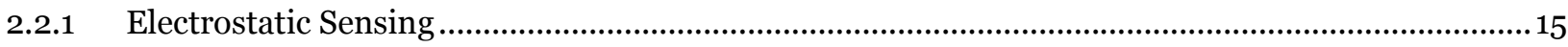

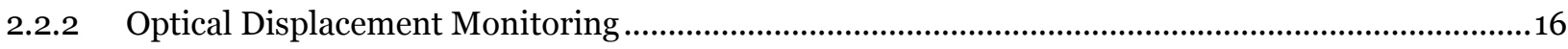

2.2.3 Enabling Doppler Laser for Displacement Monitoring of LCRs .....................................................18

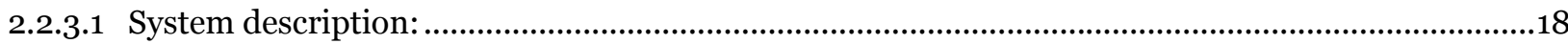

\section{Chapter 3}

\section{Automatic Control of MEMS Devices}

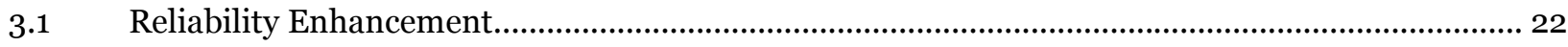

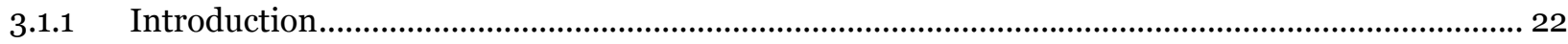

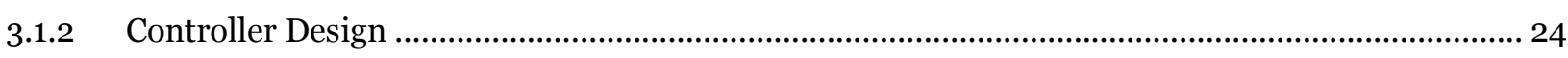

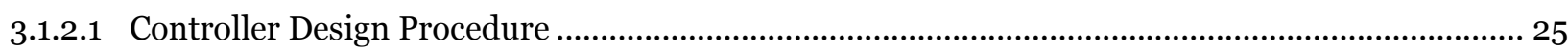

3.1.3 Reliability Evaluation: Simulation and Experimental Results ............................................................27

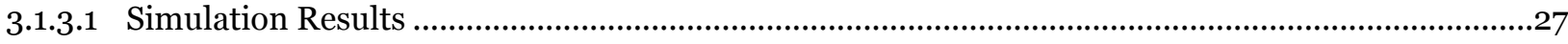

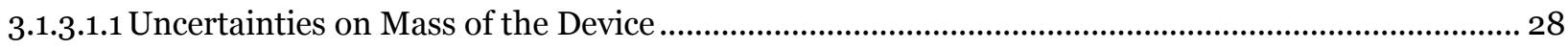

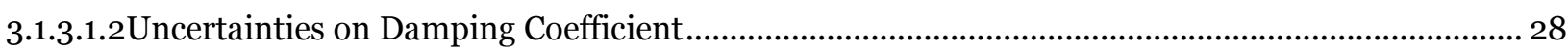

3.1.3.1.3Uncertainties on Spring Constant ....................................................................................................... 30

3.1.3.2 Experimental Results ............................................................................................................... 30

3.2 Shuttle Rotation Control of MEMS Lateral Comb Resonators Under Fault .................................. 36

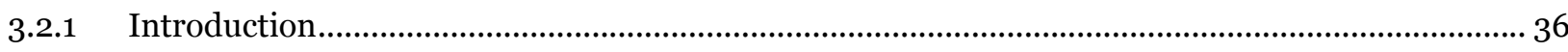


3.2.2 Problem Definition .....................................................................................................................

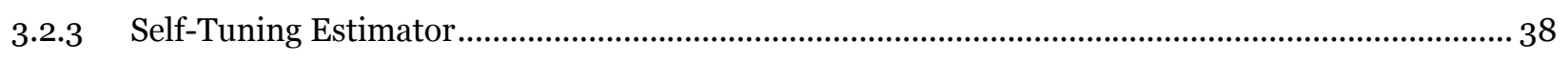

3.2.4 Self-Tuning Variable Structure Controller.....................................................................................40

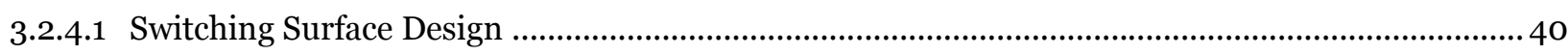

3.2.4.2 Control Law ............................................................................................................................... 41

3.2.5 Formulation for MEMS Lateral Comb Resonator ................................................................... 43

3.2.5.1 Self-Tuning Estimator............................................................................................................... 43

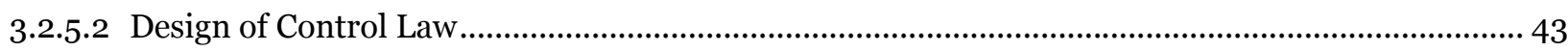

3.2.6 Experiment Setup and Rotation Monitoring ................................................................................ 44

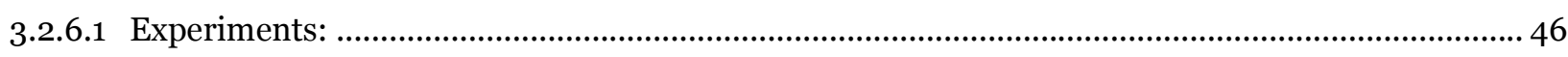

3.3 Online Parameter Identification and Control of MEMS .............................................................. 49

3.3.1 Online parameter identification: ................................................................................................ 49

3.3.2 Recursive Least Square with Forgetting Factor: ........................................................................... 49

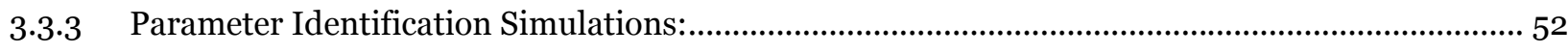

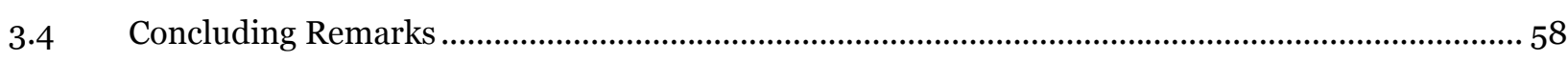

\section{Chapter 4}

\section{A New Input-Output Controller Design and Application in Control of} MEMS Comb Resonators

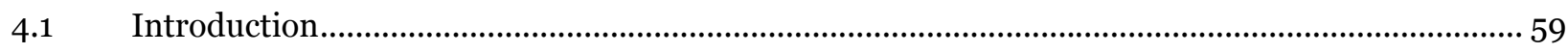

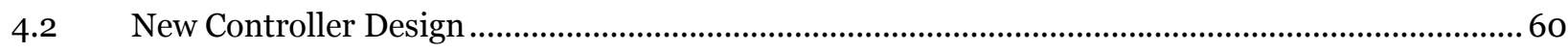

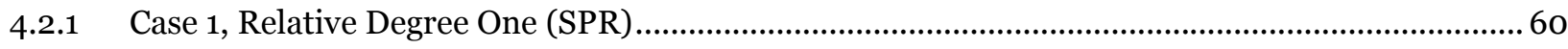

4.2.2 Case 2, Relative Degree Greater Than One ................................................................................... 63

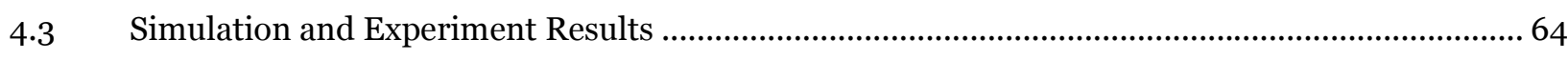

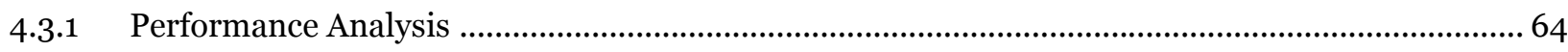

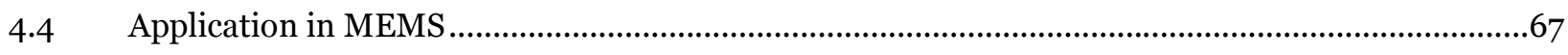

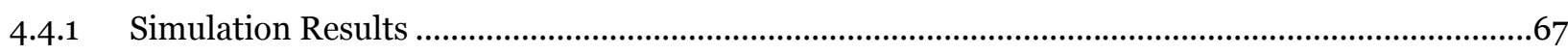

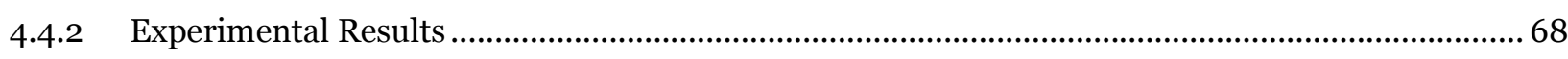

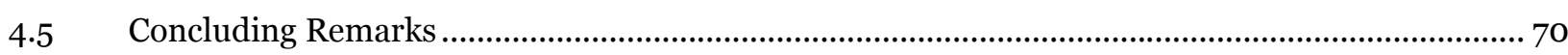

\section{Chapter 5}

\section{Intelligent Fault Diagnosis of MEMS LCR}

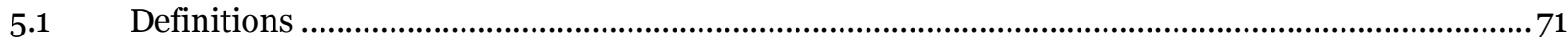

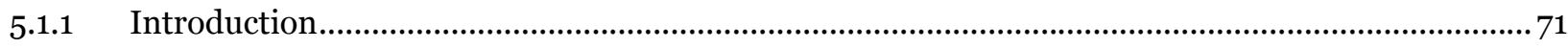

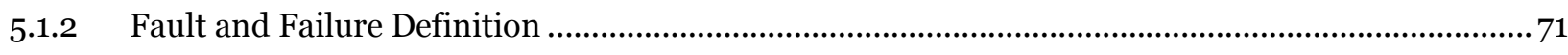

$5.1 .3 \quad$ System Behavior ................................................................................................................72

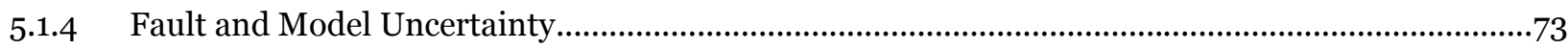

5.1.5 Faulty System Modes and Conditions:..............................................................................................

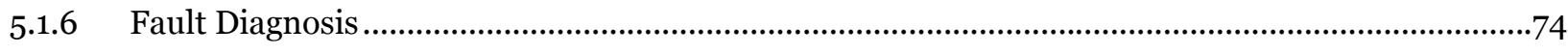

5.2 MEMS Fault Diagnosis Using Kalman Filters …….................................................................75 
5.2.1 Lateral Comb Resonator Modeling …............................................................................................76

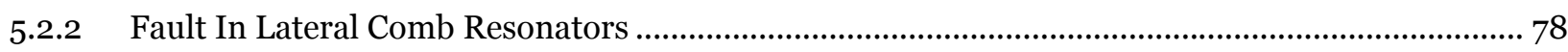

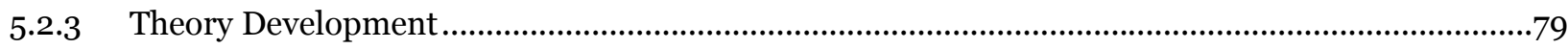

5.2.3.1 Kalman Filter Design for LCRs................................................................................................

5.2.3.2 Multiple-Model Adaptive Estimation Equations............................................................................ 80

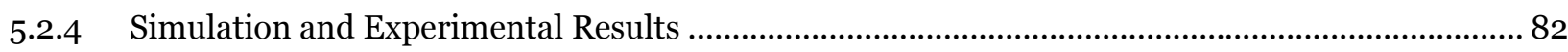

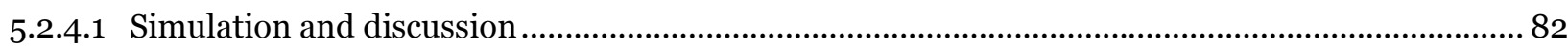

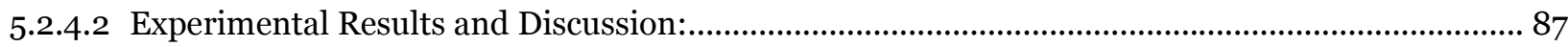

$5.3 \quad$ Self-Tuning Banks and Their Application in MEMS Fault Diagnosis............................................90

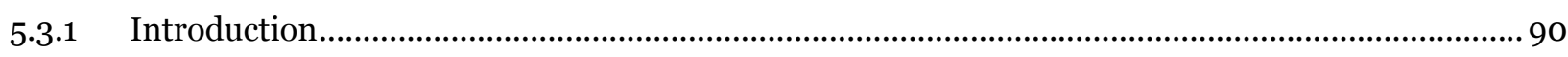

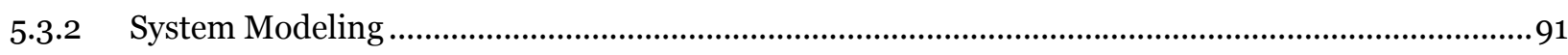

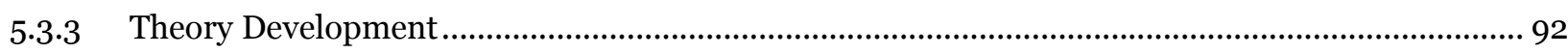

5.3.3.1 Self-Tuning Estimator ............................................................................................................. 92

5.3.4 Fault Diagnosis Application in MEMS: Simulation and Experiment ........................................... 94

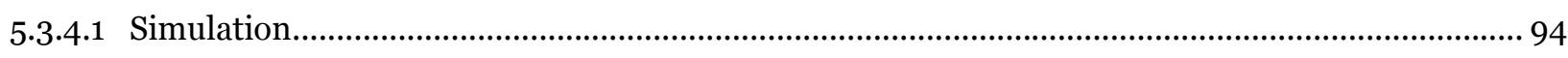

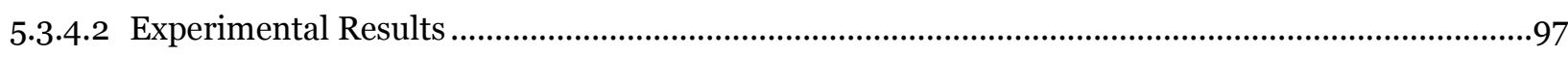

$5.4 \quad$ Adaptive Forgetting Factor ..................................................................................................... 100

5.4.1 Online implementation of Adaptive Approach for Optimal Forgetting Factor ........................... 100

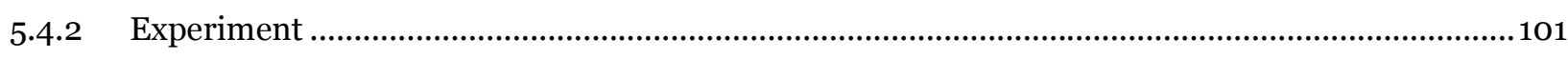

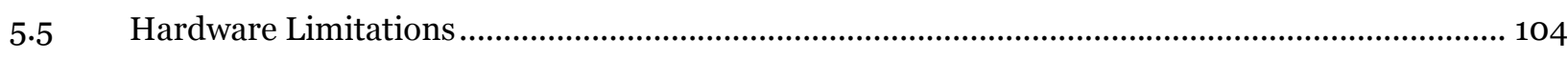

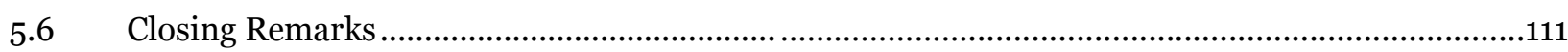

\section{Chapter 6}

\section{Future Work}

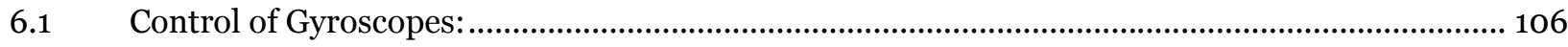

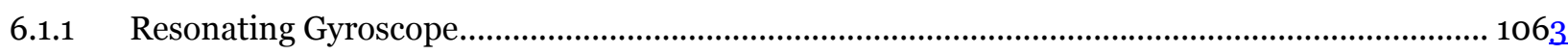

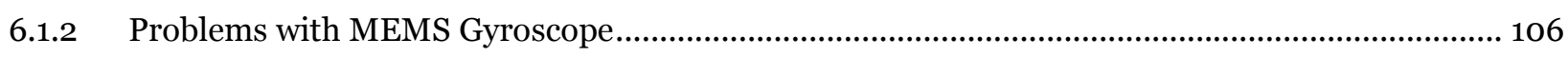

6.1.3 Imperfections in MEMS Gyro Manufacturing............................................................................107

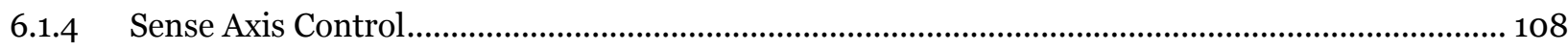

6.1.5 Drift Reduction............................................................................................................................111

6.2 Advances in Control Design of a MIMO System ........................................................................ 113

6.3 Multi Beam Optical Monitoring Technique................................................................................. 113

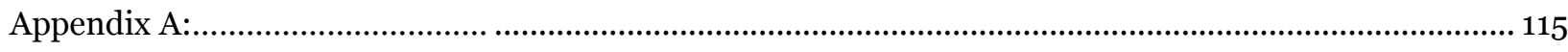

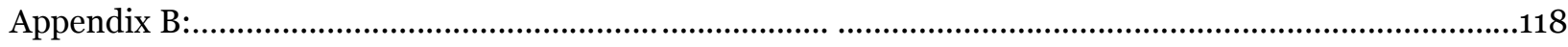

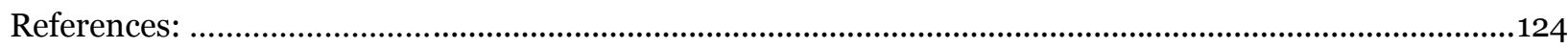




\section{Chapter 1,}

\section{MEMS Devices: Manufacturing and Parameter Uncertainties}

This chapter focuses on the MEMS Lateral Comb Resonators and their manufacturing steps. The imperfections in some of the main steps are introduced and their effects on the parameters of the system are investigated. MEMS modeling and control trends are also discussed in the end of the chapter.

\subsection{Micro Electro Mechanical System}

As the name of Micro Electro Mechanical Systems implies, they are combination of small moving mechanical parts with the electric, electronic excitation or both. MEMS can be used in many applications since they are manufactured in different sizes and shapes. The most common type of MEMS (which is also investigated in this research) is an electrostatic-excited laterally displaced comb shaped sets known as Micro Comb Resonator. Micro comb resonators are used in many applications to operate as a sensor or an actuator.

\subsection{MEMS Comb Resonators}

Shown in Figure 1.2.1, a comb resonator consists of a moving shuttle, which resonates between two sets of stationary comb-shaped parts at both sides. The electrostatic force is generated through interaction of $\mathrm{DC}$ and an $\mathrm{AC}$ voltages applied to the stationary and shuttle combs respectively. An opposite sign DC voltage is applied to other set of stationary combs to increase the applied force. The shuttle is suspended on top of the bottom plate by means of suspension springs (manufactured on the anchors). Suspension springs provide enough force to suspend the shuttle and provide a restoring force for returning the shuttle back to the rest position.

A comb resonator can be used as a sensor or an actuator. An accelerometer measures the external rotation/acceleration rates by providing the displacement-sensitive bridges along the capacitance existing between the stationary and shuttle comb fingers. As an actuator, a comb resonator drives the mass proof of the MEMS gyroscopes. 
LCRs as fundamental MEMS devices are used in many applications ranging from data storage, micro surgery, HDTV, fiber optic switching and signal attenuation, spectrometry and micro gyroscope drivers (Wamg, Hong, Brandt, Darrel \& Long. 2005), ( Ikuta, Nokota \& Aritomi. 1994), (Osiander, Firebaugh, Champoin, Farrar \& Darrin. 2004), (Fiedziuszko. -), (Shams, Moniuszko \& Ingham. 2001), (Dellman, Akaiyama, Brian, Gautsch, Guenat, Guldimann, Luginbuhal, Marxer, Staufer, DerSchoot \& Rooij. 200o).

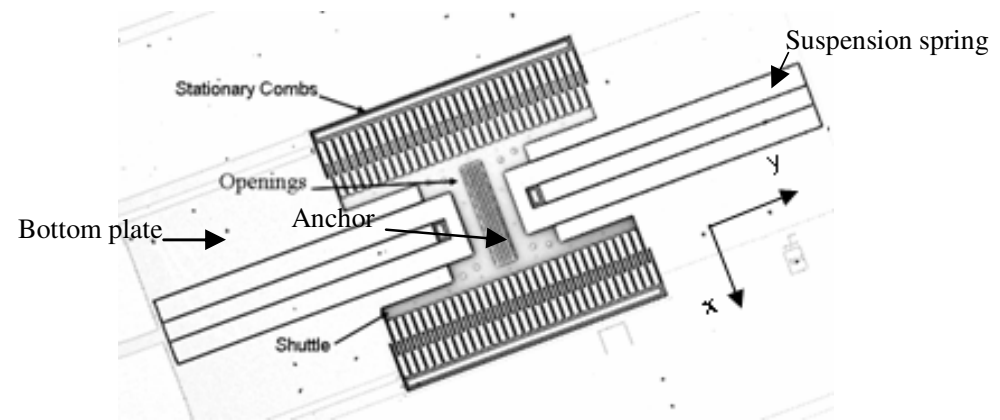

Figure 1.2.1: Lateral comb Lateral Motion Resonator

\subsection{LCR Model}

The mathematical model of MEMS helps to present a better understanding of behavior of microsystem. Parameter recognition opens a window to unknown phenomena affecting the performance of microsystem; however, a complete and precise value of the system's parameters cannot be achieved and presented by mathematical models. MEMS devices in a simple form are modeled as a mass spring damper system, shown in Figure 1.3.1, with time and condition-varying parameters. A second order differential equation which governs the behavior of the system is

$$
F_{e}=m \ddot{x}+\beta \dot{x}+2 k_{s} x+F_{d},
$$

where $m$ is mass content of the center comb or shuttle, $x$ is the displacement, $k_{s}$ is the spring constant on one side of the device, $\beta$ is the damping coefficient, and $F_{d}$ is force of the load.

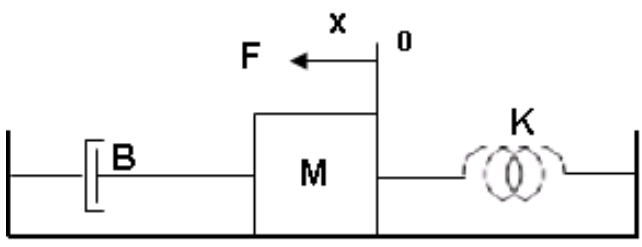

Figure 1.3.1: Mass-Spring-Damper system.

The main direction of movement is along the x-axis, as it is shown in Figure 1.3.1, the effective 
value of each parameter is calculated along the main direction of displacement (x).

The electrostatic force generated by applying the body and shuttle voltages is calculated as:

$$
F_{e}=-4 n \varepsilon \frac{t}{g}\left(V_{b} \cdot V_{s}\right)
$$

where $n$ is the number of comb fingers, $\varepsilon$ is the permittivity of air with $\left(\varepsilon_{0}=8.854 e-12\left(C^{2} / N . m^{2}\right)\right)$, $t$ is the vertical thickness of the fingers, $g$ is the gap between stator and translation comb fingers, $V_{b}, V_{s}$ are DC voltage applied to the stator combs and AC voltage applied to the translation stage (shuttle) respectively. A valid model contains accurate values for parameters, but it should be noted that these parameters differ in the fabricated devices from their initial and expected values.

\section{Mass Calculations:}

The mass content of the stage can be calculated, considering of polysilicon deposition density $\left(2.33 e-15\left(\mathrm{~kg} / \mu \mathrm{m}^{3}\right)\right)$ and the volume of the shuttle. More accurate computations considering the effective direction of movement (x-axis) takes more details of the system into account and is expressed as

$$
m_{x}=m_{s}+\frac{1}{4} m_{t}+\frac{12}{35} m_{b}
$$

where $m_{s}$ is the mass of the stage, $m_{t}$ the mass of the flexure trusses, and $m_{b}$ is the mass of the flexure beams. Dimensions and parts are shown in Figure 1.3.2.

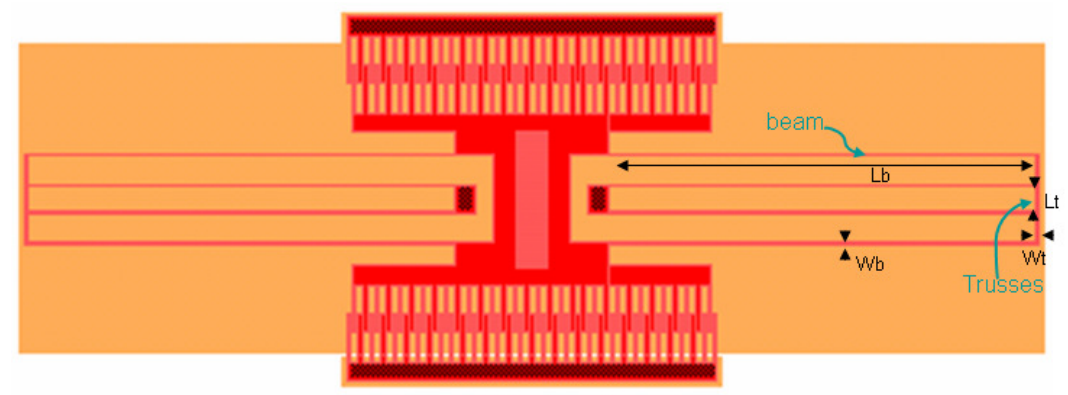

Figure. 1.3.2.

\section{Spring Constant Calculations:}

The folded spring constant of the device is more effective along the x-axis, however, there is 
components along other directions as well. The effective spring constant in the x-axis can be obtained as

$$
k_{x}=\frac{2 E t w_{b}^{3}}{L_{b}^{3}} \cdot \frac{L_{t}^{2}+14 \alpha L_{t} L_{b}+36 \alpha^{2} L_{b}^{2}}{4 L_{t}^{2}+41 \alpha L_{t} L_{b}+36 \alpha^{2} L_{b}^{2}},
$$

and

$$
\alpha=\left(\frac{w_{t}}{w_{b}}\right)^{3},
$$

where $E$ is the Young modulus, and other dimensions are shown in the Figure 1.3.2. an average value was used for the trusses length and a unity value for $\alpha \approx 1$. The resonant frequency of the device considering the mass calculated from (1.3.3) and the spring constant from (1.3.4) results in resonant frequency as

$$
\omega_{R}=\sqrt{\frac{k}{m}}
$$

\section{Damping Coefficient:}

Damping effect arises from the energy dissipations of a moving plate in a viscous material. Damping is modeled in two ways known as Couette flow and squeeze film. Couette damping occurs when two parallel plates are moved laterally on top of one another as shown in Figure 1.3.3 (a). The squeeze film effect occurs when the plates move towards each other and press the surrounding material out of the volume shown in Figure 1.3.3 (b).

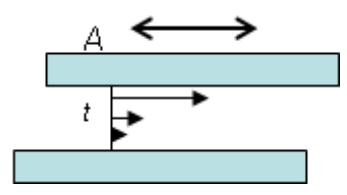

(a)

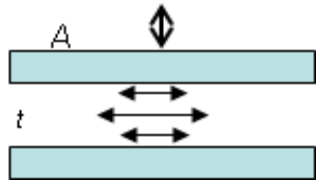

(b)

Figure 1.3.3. The damping coefficient in different movement directions.

The Couette damping coefficient is computed as

$$
\beta_{C}=\frac{\mu A}{t},
$$

where $\mu$ is the surrounding gas viscosity, $A$ is the plate area and $t$ is the distance between the plates or the gas film thickness. The squeeze film is more effective along the direction of movement ( $x$-axis) and is calculated as

$$
\beta_{x}=\mu\left[\left(A_{s}+\frac{A_{t}}{2}+\frac{A_{b}}{2}\right) \cdot\left(\frac{1}{t}+\frac{1}{\delta}\right)+\frac{A_{c}}{g}\right]
$$


where $A_{s}$ is the area of the stage, $A_{t}$ is the surface area of trusses, $A_{b}$ is the surface area of the flexure beams, $\delta$ is the penetration depth of the airflow above the stage, $A_{c}$ is the surface area of the comb finger sidewalls and $g$ is the shuttle finger to stationary finger gap.

As illustrated, the effective mass, spring constant and damping coefficients are related to small details in the structure of the device. Geometry of the device, however, is highly affected by the manufacturing steps. These effects are accelerated when the device operates in the open air and is exposed to humidity, dust and temperature variations. Manufacturing tolerances for a specific manufacturer are listed in table 1.1 as an example.

Table 1.1: Parameter variation of a LCR shown in Figure 1.2.1, (Wang. 2004)

\begin{tabular}{|c|c|c|c|c|c|c|c|c|c|c|}
\hline \multirow{2}{*}{\multicolumn{2}{|c|}{\begin{tabular}{c|c} 
Structure & $\begin{array}{c}\text { Beam } \\
\text { length } \\
(\mu \mathrm{m})\end{array}$ \\
\end{tabular}}} & \multicolumn{3}{|c|}{$\begin{array}{c}\text { Mass } \\
\left(1 \theta^{t \theta} \mathrm{kg}\right)\end{array}$} & \multicolumn{3}{|c|}{$\underset{K}{\text { Spring constant }_{K}}$} & \multicolumn{3}{|c|}{$\begin{array}{c}\text { Resonant frequency } \\
(\mathrm{Hz})\end{array}$} \\
\hline & & $\operatorname{Min}^{*}$ & Std. & $\mathrm{Max}^{*}$ & $\operatorname{Min}^{*}$ & Std. & Max* & $\operatorname{Min}^{*}$ & Std. & Max* \\
\hline \multirow{4}{*}{ Grating } & & & & & & & & & & \\
\hline & & & & & & & & & & \\
\hline & 450 & 1.9331 & 1.9459 & 1.9844 & 0.0132 & 0.0252 & 0.0441 & 1724.8 & 2563.1 & 3115.3 \\
\hline & 500 & 1.9675 & 1.9587 & 2.0013 & 0.0097 & 0.0186 & 0.0325 & 1478.9 & 2194.8 & 2666.3 \\
\hline
\end{tabular}

These values show that for a fabricated device, small variation in operating conditions and manufacturing tolerances in parts of the structure result in parameter variations in the final product. Bode plots of an example system with upper and lower boundary values are shown in Figure 1.3.4. Upper limits of parameters represent either stiffer spring constants or massive shuttles, which cause higher resonant frequencies while lower limit values refer to lower resonant frequencies. In this research, the parameters of the device are considered as unknown values, and online parameter identifiers (estimators) are used to estimate the parameters of the system. In this regard, considering the uncertain parameter values for the system shown by a $\left(^{\wedge}\right)$ sign, the governing equation of the system is expressed as

$$
\hat{F}_{e}=\hat{m} \ddot{x}+\hat{\beta} \dot{x}+2 \hat{k}_{s} x+F_{d} .
$$



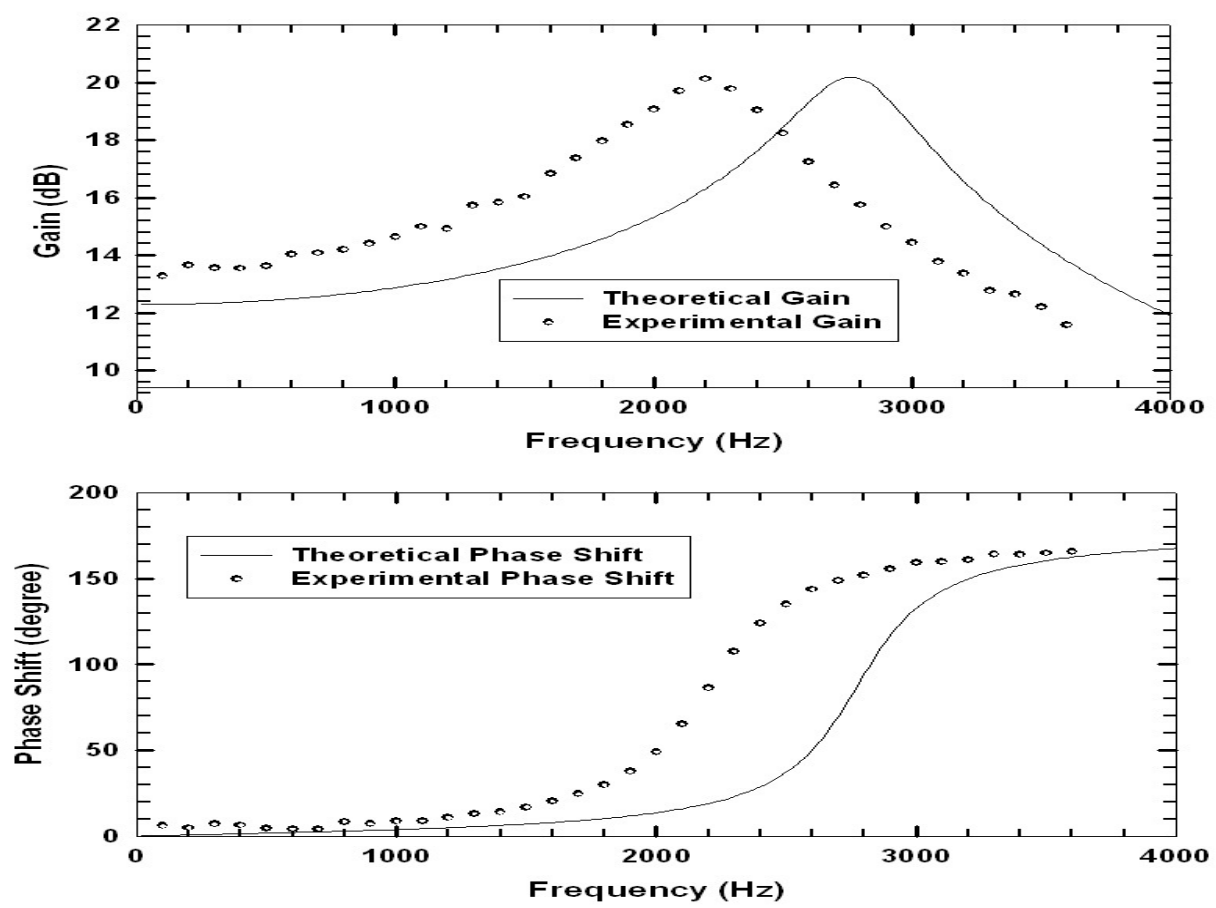

Figure 1.3.4: The shift in the phase and amplitude of LCRs. Deviation between the manufactured and desired expected system (Wang. 2004)

\subsection{MEMS Parameter Uncertainties and Manufacturing Steps}

MEMS devices are manufactured in different techniques, one of which is using the sacrificial layers made mainly from silicon oxide which are removed to free the layers from one another. Different manufacturing steps and several machining stages are required for bulk and surface micro machining. Machining procedure on the areas larger than $10 \mu \mathrm{m}^{2}$ are called bulk micromachining and those on smaller areas are called surface micromachining. Layers, parts, joints and containers in MEMS should possess specific mechanical and electrical characteristics. The problem occurs when there is an imperfection in the machining procedure, which results in different parameters than those expected in the design of the device. Devices with parameters other than expected are hard to control and this matter is even worse when the parameters vary from device to device and are influenced by environmental and operating conditions. In the following sections of this chapter, the effects of the manufacturing steps on the surfaces, areas, sidewalls, body shape and their resultant parameter variations are investigated.

\subsubsection{Material Growth Effects}

Silicon in different forms and configurations are widely used in MEMS structures. These material form different parts of a device and are deposited generally in high temperature and different deposition rates, resulting specific mechanical and electrical characteristics. Material growth usually requires high temperatures which might change the properties of the other 
deposited parts and interfacing circuits. Polysilicon, for example, contains of several domains with different crystalline orientations called 'grains'. The orientation and size of these domains change by heat processing. This might affect the electrical and mechanical properties from those expected. Several fabrication steps are used in MEMS manufacturing, which affect the parameters of the device, some of them are investigated in the following sections.

\subsubsection{Lithography and Its Effects}

Photolithography is one of the key manufacturing steps in fabrication of MEMS devices, which includes covering of specific areas for micromachining. This includes mask alignment, UV exposure, baking, and photoresist removing for fabrication. Mask is a pattern which covers some parts of the device for microfabrication. Manufacturing of different parts require different photo masks. For implementing of a symmetric design on silicon wafer and creating mechanical devices, very accurate techniques are required. Masking as a part of photolithography process is one of the critical steps which locates the casts of different parts of a mechanical device in the substrate. The quality of final product and symmetry of the structure mainly depends on the precision of this step. Figure 1.4.2.1 illustrates a catastrophic masking error, which has joint the comb fingers of the stationary and shuttle together. In a simpler case, this might just be observed as a very small asymmetry in the device, which leaves the lateral displacement unequal on both sides.

In bulk micro machining, alignment of MEMS device pattern (photomask) with crystalline orientation of substrate is also important in many cases to have the surface of the grown material smooth. Any rotation from the required orientation will result in 'knife effect' meaning that the surface is chopped. These types of surfaces may contain deep cracks and sharp edges in higher rotation angles. The knife effect is illustrated in Figure 1.4.2.2 and the effect of higher rotation angles is shown in Figure 1.4.2.3.

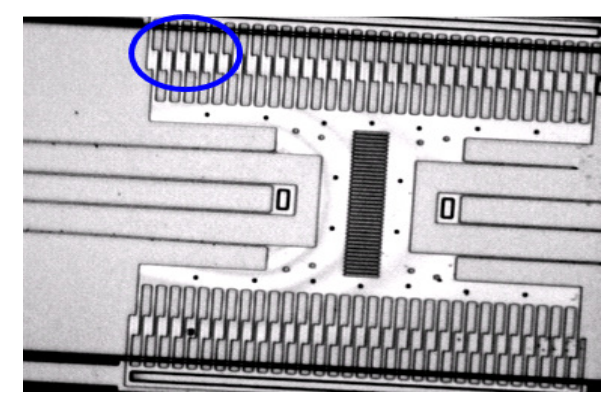

Figure 1.4.2.1: the fingers of the stationary part and the lateral shuttle joint together with lithography defects.

In general, lithography can be considered as one of the critical steps in manufacturing of the MEMS devices. Parameters such as damping coefficient and spring constant largely vary by small fabrication errors. For instance, a $0.2 \mu \mathrm{m}$ reduction in the size of a $5 \mu \mathrm{m}$-wide spring beam is a $4 \%$ reduction in the width of the spring and results in approximately a $12 \%$ change in spring constant, 
whereas for a $1 \mu \mathrm{m}$-wide spring, reduced by the same amount, the change is $20 \%$ which result in 49\% spring constant variations (Gad-el-Hak, M. 2001).

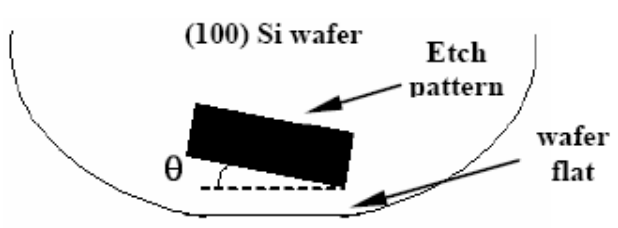

Figure 1.4.2.2: the miss-alignment angle from the wafer orientation (Reddy \& Jessing. 2004).

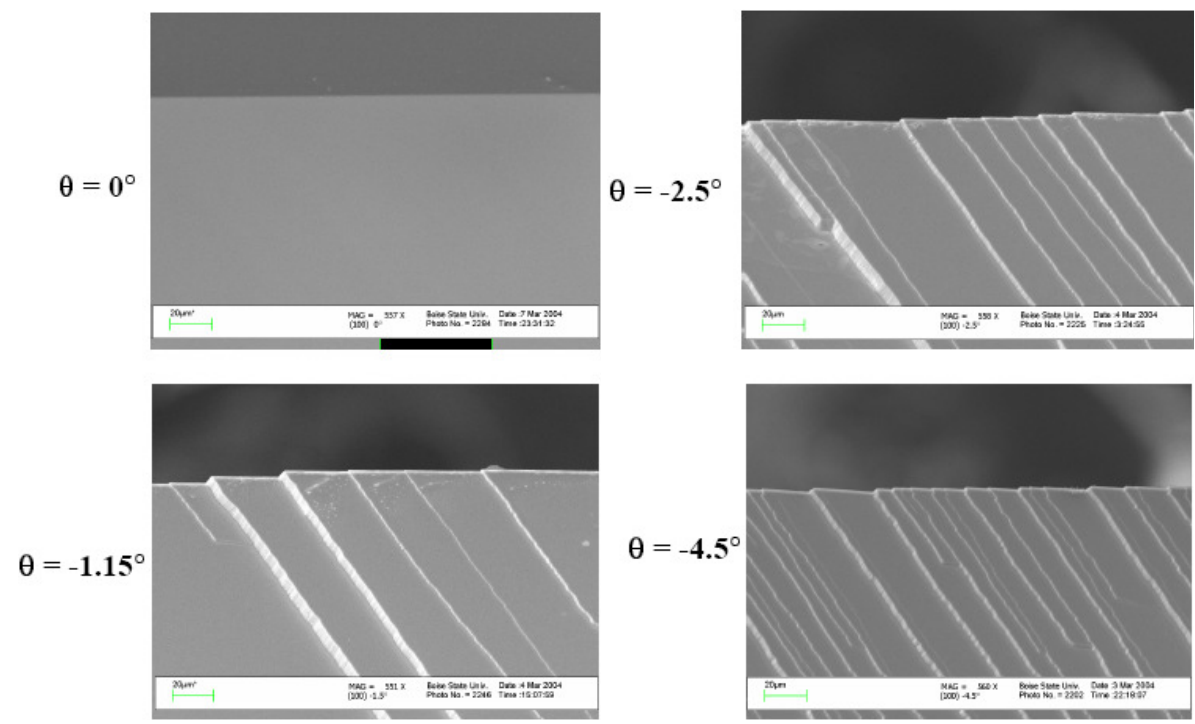

Figure 1.4.2.3: The miss-alignment effect in different angles of bulk micromachining or knife effect (Reddy \& Jessing. 2004).

\subsubsection{Effect of Etching Process on Parameters of MEMS}

Etching is defined as removing of a specific material in a specific direction/rate which can be done by liquid or gaseous material known as etchants. These materials dissolve the targets in two different ways, isotropic and anisotropic, both of which require that the end of the etching process be recognized. Isotropic etchants are those that etch all different surfaces at the same rate whereas anisotropic which has different etching rates at different directions. In this way, some selective surfaces are etched more.

Anisotropic etchants usually leave a surface too rough in macro-scale. The macroscopic roughness, named as notching or pillowing are observed when the center of the etched area has a slower average etching rate compared to the borders of that area. The difference can be as large as 1-2 $\mu \mathrm{m}$, which is considerable in a 10-20 $\mu \mathrm{m}$ etch length. Over and under-etching deforms the cantilevers, spring beams, and surfaces and changes the critical values of the microsystem which for instance can be observed as shift in the resonant frequency of the device. Figure 1.4.3.1 shows 
the notching and roughness effects (Gad-el-Hak. 2001).
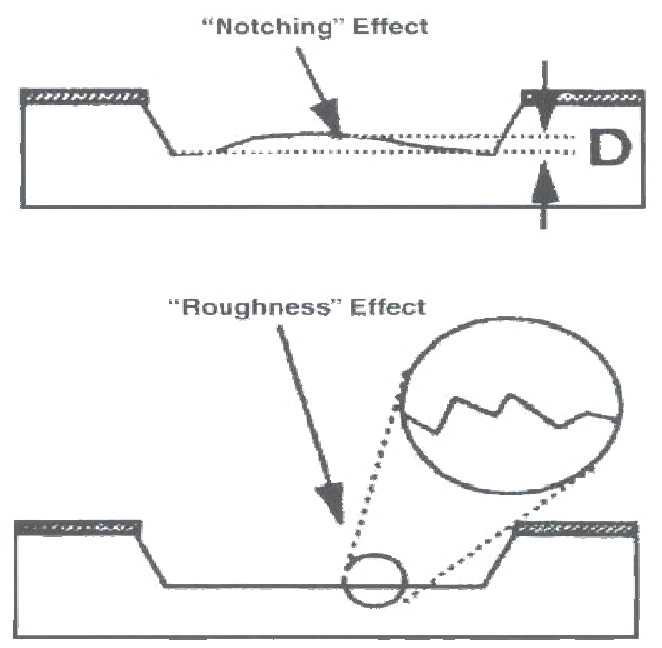

Figure 1.4.3.1: Macroscopic and microscopic roughness (Gad-el-Hak. 2001).

\subsubsection{Effect of Stress, Stress Gradient, Wearing and Stiction in Deposited Films}

Stress: One of the most important sources of nonlinearities is the stress in thin films. Stress is originated from different sources such as differences in thermal expansion coefficient of different layers and materials, specifically when a thin film is deposited at a higher temperature than other layers. Another source of stress is the difference in size of dopant atoms in a thin film layer. For example, the differences in size of thermally grown silicon dioxide $\left(\mathrm{SiO}_{2}\right)$ cause compression, because the size of oxide atoms is larger than the lattice size in substrate silicon atoms. Very large stress values may result in cracking or delaminating of films and layers. Stress in films can be observed as strain or deformation of microstructures. Other effect of stress is observed as the change of the resonant frequency of the device as shown in Figure 1.3.4. In a comb resonator containing different layers which are deposited individually at different annealing and thermal processes, higher stress values are expected. Therefore, temperature variations and environmental conditions might change the device's behavior by directly affecting its parameters.

Stress Gradient: Changing the stress values by thickness of the layers, which is called stress gradient, adds up to nonlinearity of thin films. High stress gradients on thin plates and gears result in deformation of spiral shape beams.

Wearing: When two surfaces rub against each other, the friction between them makes them wear. Friction at the microscopic level is similar to that of macro-size bodies. If surfaces are made of the same material, they even show more friction and wear faster. In conventional designs, surfaces have different materials to reduce the friction. In most of MEMS devices the surfaces that rub against each other are made of silicon or polysilicon, and most of the time they operate dry. Usually, devices operating at frequencies higher than the resonant frequency wear faster. The 
wear rate is a function of adhesion of surfaces rubbing against each other. The wearing of the device accelerates when surfaces start decaying. Therefore, lifetime of the device is limited by friction and operating conditions such as operating frequency and dryness.

Stiction: One of the most harmful effects on the MEMS device is stiction, which is unintended absorption of surfaces and parts. The effect of stiction is shown in Figure 1.4.4.1.

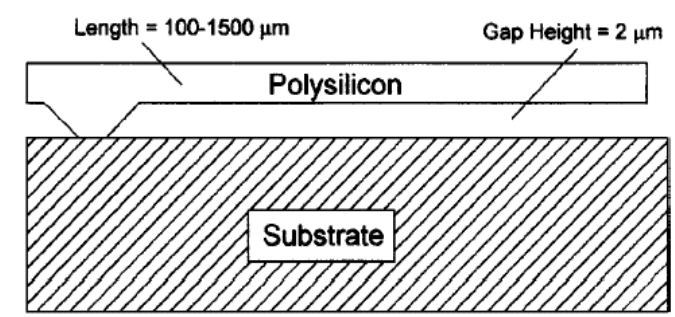

(a)

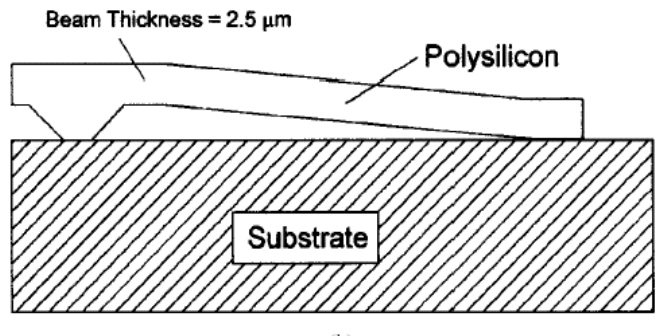

(b)

Figure 1.4.4.1: Stiction failure: (a) free-standing cantilever; (b) stiction of the same cantilever (Rogers\& Phinney. 2001)

The factors that cause stiction are nano-scale surface roughness, large surface areas, electrostatic attraction, humidity and hydro-absorbent surfaces. Two main forms of stiction are stiction due to release process and "in-use" stiction (Gad-el-Hak. 2001). The main cause of stiction in the release process involves capillary forces of the etchants and other washing liquids that pull the surfaces and parts together as shown in Figure 1.4.4.1. Trapped charges on the surfaces can cause electrostatic attraction and increase the stiction. In addition, an extremely humid environment increases the adhesion energy of the parts and elevates the stiction. Water has the same effect as that of the release process on the adhesion of the parts. Stiction effect is increased after acid releasing process and dissolving of the sacrificial layers.

\subsection{Electrostatic Force}

The electrostatic field around the opening areas experiences field fringing, which affects field uniformity and causes forces. The force is affected by distance between the surfaces and shape of the bodies facing each other. Much of the related research has neglected the effect of fringing fields, although several researchers have attempted to devise a lump formula for it, which considers merely the geometry of the plates (Gyimesi, Avdeev \& Ostergaad. 2004), (Leus \& Elata. 2004), (Wu, Zhe, Wang, Cheng, Modi \& Farmer. -). The main effect of fringing is the change of 
electrostatic force according to the applied voltages. Another effect in MEMS device involves the pull-in voltage. According to inversely proportional relation of the electrostatic force to distance of the parts, if the parts are placed closer than $67 \%$ of total air gap between them, then the increment rate of electrostatic force becomes greater than that of the suspension spring restoring force which puts the device into unstable region of operation. In motion control of MEMS devices, some of the control efforts are to neutralize the unwanted forces in the device. Asymmetry of MEMS structure and devices especially designed for torsion purposes cause production of unequal electrostatic force on the bodies. This matter dominates the pull-in voltage effect. However, microscopic surface roughness in the large distances between surfaces have no significant effect on the electric field distribution of the surfaces.

Humidity of air surrounding the device increases the stiction of plates and contributes to unwanted forces, hence, in micro-scale devices, the electrostatic force is a nonlinear function of the quality of interface surrounding material between the plates, shape and distance between fingers, release process, roughness of the bulk substrate, and applied voltage. These effects and parameter-variations cannot be expressed in a lump formulation of electrostatic force, resulting uncertain variation of the force during operation. Table 2.1 briefly introduces the parameter variations and their causes in the microfabrication processes.

Table 2.1: Direct Effect of Manufacturing Process and Mechanical Effects on the Parameters of the Device

\begin{tabular}{|c|c|c|c|c|c|c|c|c|}
\hline & Lithography & $\begin{array}{c}\text { Material } \\
\text { Growth }\end{array}$ & Etching & $\begin{array}{c}\text { Mechanical } \\
\text { Properties }\end{array}$ & $\begin{array}{c}\text { Surface } \\
\text { Roughness }\end{array}$ & Stress & Stiction & Aging \\
\hline Mass & & $\bullet$ & & $\bullet$ & & & & \\
\hline $\begin{array}{c}\text { Spring } \\
\text { Constant }\end{array}$ & $\bullet$ & $\bullet$ & $\bullet$ & $\bullet$ & & $\bullet$ & & $\bullet$ \\
\hline Damping & $\bullet$ & & $\bullet$ & & & & & $\bullet$ \\
\hline $\begin{array}{c}\text { Electrostatic } \\
\text { Force }\end{array}$ & & & $\bullet$ & & $\bullet$ & & \\
\hline
\end{tabular}

\subsection{MEMS Control Trends}

The focus of this research is on the control of the MEMS device considering their parameter uncertainties and fault conditions. Different configurations of controllers are considered for the control of the trajectory of the MEMS comb resonators in different types of fault occurrence in their system. Different types of fault are physically manufactured and tested for the control of shuttle locations. Previous research show the application of off-line system identifiers combined with controllers. The application of digital controllers to compensate for the imperfect structure of gyroscopes has also been studied. In this research, the continuous time adaptive controllers are designed and implemented in real time control board dSPACE DS1103. The feedback of the location of the shuttle is provided using optical technique called through wafer optical monitoring. A survey shows different types of controllers have been applied for MEMS control 
purposes ranging from open loop and PID control to advanced controls such as sliding mode, feedback linearization, and adaptive techniques (Wang, Dawson, Hornak, Famouri \&Ghaffarian), (Chueung, Horowitz and Howe. 1995), (Chen, Famouri, \& Hornak. 1999), (Dawson, Wang, Chen, Famouri \& Hornak. 2000), (Park, Horowitz \& Tan. 2001), (Wang. 2004), (Wu, Zhe, Wang, Cheng, Modi \& Farmer. - -). 


\section{Chapter 2,}

\section{Control Concept and Displacement Feedback Techniques}

In this chapter, the principle of the control approach is investigated. The concept of Adaptive control in forms of Model Reference and Self-Tuning are introduced and developed for MEMS applications. Other control techniques are also applied for specific problems observed in MEMS. The principles of displacement monitoring in form of through wafer monitoring and capacitive measurement are also illustrated in this chapter.

\subsection{Introduction to Control}

Micro Electro Mechanical Systems are a very important part of the sophisticated applications and are used to enhance the functionality of specific devices under different conditions. As mentioned earlier the time and condition varying parameters of the MEMS suggests for an active control to reach high performance operations and controls. Adaptive controllers provide robust control systems with high trajectory control performances. Following sections investigate the control of the MEMS under fault conditions; adaptive control approach provides better performance than that of robust control which requires a known boundary of parameter variations. The structure and concept of adaptive control methods used in this research are introduced in the next sections.

\subsubsection{Adaptive control}

Adaptive control is defined as the combination of a parameter estimator and a control law for a class of systems with unknown or time-varying parameters. Different techniques of parameter estimation, control laws and their combination provide various types of adaptive controllers. In this research, the direct and indirect adaptive control approaches are investigated. Direct adaptive 
control or Model Reference Adaptive controllers adapt the gains of the controller without the need for exact values of the system parameters. In the indirect or self-tuning adaptive control technique, the gains of the controller are determined through a function which relates the system parameters to the controller gains. In this approach, an estimation of the system parameters is required that can be obtained by parameter identification techniques. The self-tuning controller (estimation based) can be combined with any other type of control law. This allows the application of very robust variable structure controllers combined with system identification units.

\subsubsection{Model Reference Adaptive Controller}

The structure of a Model Reference Adaptive Controller (MRAC) is introduced in Figure 2.2.1 where $G_{m}$ is the model's transfer function, $G_{p}$ is the plant's transfer function, and u is the control command. A reference input is applied to the model and generated output is the model reference. The output of the plant is compared with that of the model where the error in between is called tracking error. The adaptation unit generates suitable gains for controller to have the plant controlled by the model reference output. The main concern in this controller is the relative degree of the plant and order of the system. Different approaches are taken for systems with relative degrees higher than one and none strictly positive real systems (See Appendix A). The control law and adaptation techniques are more described in details in chapter 3.

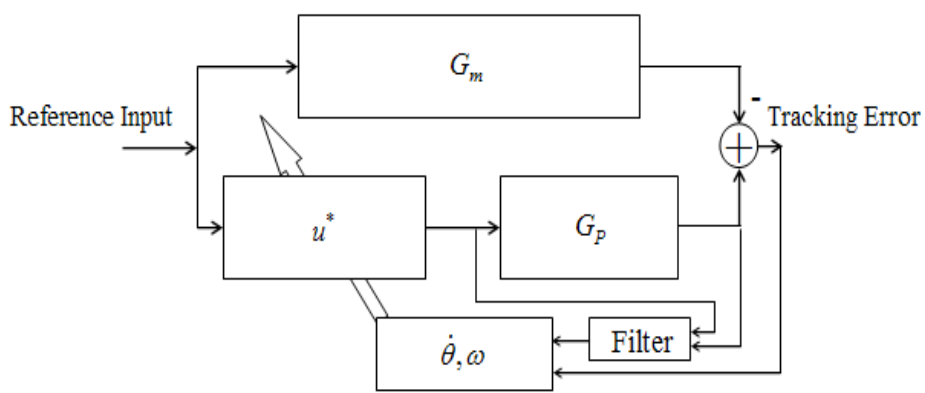

Figure 2.2.1, Model Reference Adaptive Controller for Strictly Positive Real Systems.

\subsubsection{Self-Tuning}

Self-tuning controllers have online parameter estimators and control parameter computation units. The controller gains are computed indirectly and require the estimated parameters of the system, so that the controller is called indirect or self-tuning. Figure 2.2.3.1 shows the self-tuning controllers structure. 


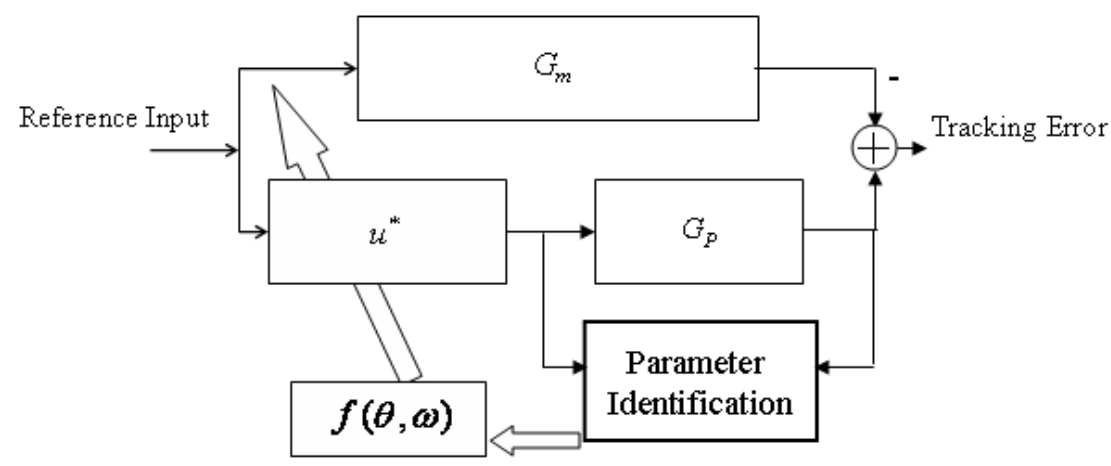

Figure 2.2.3.1, Self tuning control system

As it is seen the parameter identifier estimates the parameters of the plant, then the function $f(\theta, \omega)$ computes the gains of the controller. The self-tuning techniques require persistently exciting signals of a suitable degree meaning that the signal in the input and output of the plant should contain enough variations. There are different types of identifiers which are used in different cases and systems.

\subsection{Displacement Feedback Techniques}

Displacement of MEMS LCR can be measured by different techniques such as capacitive sensing and laser optical beam. Capacitive measurement technique is based on the variations in capacitance between moving parts. In this technique, an extra capacitive shape structure is required to transform the displacement into electrical signals. Other techniques make use of optical methods for measuring of displacement. The main approach applied in this proposal is focused on the through wafer optical measurement technique. The displacement information which is encoded optical signal is extracted through a data recovery unit. These techniques are illustrated in the following sections.

\subsubsection{Electrostatic Sensing}

In MEMS Micro Comb Resonators, the stationary comb fingers and those of the shuttle provide sets of capacitance which are ideally equal at both sides. When the shuttle is displaced, the balance of capacitance at both sides is disturbed. The capacitance variation is directly proportional to the displacement of the shuttle. Considering the capacitance value at the rest position as $C_{s 0}$, the displacement $x$ causes an increment of $\frac{\partial C_{s}}{\partial x} x$ to initial value and decreases the same amount from the other side as $-\frac{\partial C_{s}}{\partial x} x$. Therefore, the capacitance values after $x$ micron displacement are 


$$
\begin{aligned}
& C_{s p}=C_{s 0}+\frac{\partial C_{s}}{\partial x} x \\
& C_{s n}=C_{s 0}-\frac{\partial C_{s}}{\partial x} x
\end{aligned}
$$

where $\left(C_{s p}\right)$ is the positive (increment) and $\left(C_{s n}\right)$ is the decremented values. Figure 2.3.1.1 shows the capacitance variation and displacement measurement.

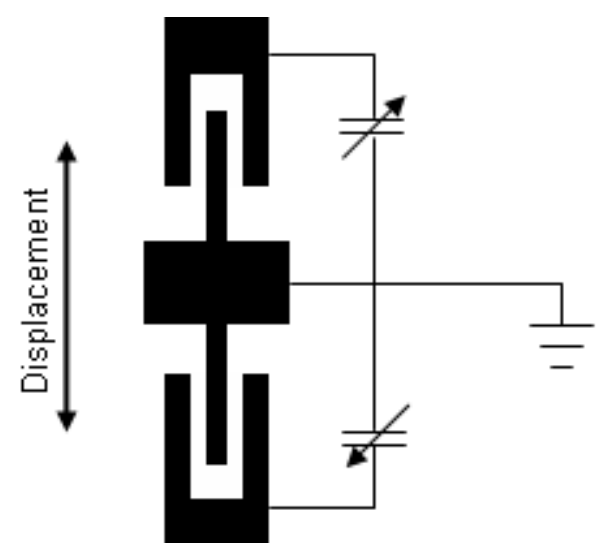

Figure 2.3.1.1, Electrostatic Sensing

\subsubsection{Optical Displacement Monitoring}

The displacement of the device can be measured through the optical waveform variation passing through the moving parts. Optical beam can be produced by a laser source at wavelength $1310 \mathrm{~nm}$ or by a Doppler laser beam. Figure 2.3.2.1 shows the through wafer monitoring technique with a laser source.

The laser beam can pass through the openings on the shuttle of MEMS and is blocked elsewhere, which produces pulses that contain the displacement information. The optical encoded waveform requires a data recovery unit designed by (Wang, 2005) to extract the location of the shuttle instantaneously. The through wafer optical monitoring system consists of a laser wave-guide emitted from a laser diode. 


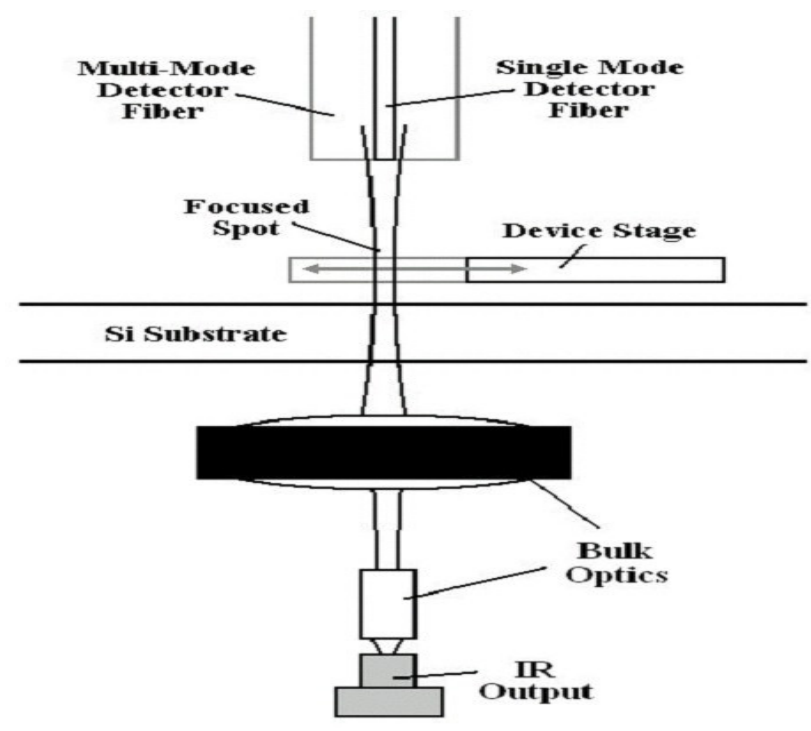

Figure 2.3.2.1: Through waver optical monitoring, multi mode detector fiber and die position (Dawson, 2004).

Two bulk lenses used to concentrate the laser beam and let it cross through MEMS die substrate which is fully polished by the polisher of 0.1 micron in order to reduce the laser beam chattering and reduction in beam loss. The laser coming out of the die is collected and conducted to a detector through a multi-mode detector fiber optic probe which houses a single-mode optical fiber. As the shuttle "center comb" moves, its body cuts the laser beam through the openings and produces pulses. The detector then experiences the intensity changes of the beam due to the shuttle displacement, therefore the output voltage of detector contains displacement information of shuttle.

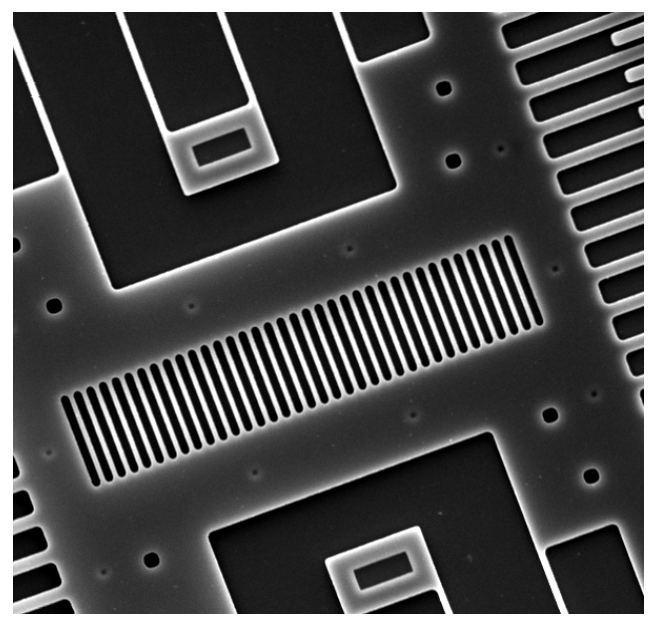

Figure 2.3.2.2: The openings on the moving stage, the concentration of the laser beams on the device and the direction of the movement. 
The alignment of output laser beam emitter and detector fiber optic collector is a critical point, which allows the maximum beam being collected to increase the Signal to Noise Ratio (SNR). The 2-micrometer wide openings, which let the laser beam cross through, are shown in Figure 2.3.2.2.

\subsubsection{Enabling Doppler Laser for Displacement Monitoring of LCRs}

The displacement auto-recovery unit, described in previous section, can extract the displacement from the peaks and valleys on the optical waveforms. The optical waveform can be obtained from through wafer or Doppler Laser system. Through wafer optical monitoring technique requires polishing of the die to decrease the beam scattering; in addition, the alignment of beam on the openings of shuttle is challenging.

\subsubsection{System description}

A Doppler laser velocimeter can be used to optically monitor the lateral displacement of microsystem. The Doppler laser measurement system uses a reference beam and a measurement beam. The variation in reflected measurement beam phase, relative to that of the reference beam, returns a signal proportional to the velocity of the surface reflecting the measurement beam relative to the surface reflecting the reference beam.

These changes in the velocimeter signal that arise from the translation of a grating structure in the LCR shuttle through the measurement beam are used to infer position information. This motion is shown in Figures 2.3.3.1.1.a, b and the optical beam placement on the device is shown in Figure 2.3.3.1.2. Given that the velocity measured by this velocimeter system corresponds to motion in the direction parallel to the beam propagation, prominent transitions in the velocimeter signal occur only as the edges of the released polysilicon layer pass through the measurement beam. This generates the peak-valley points in the optical data shown in Figure 2.3.3.1.3 and are further used in the optical data-recovery unit which measures the shuttle displacement instantaneously. 


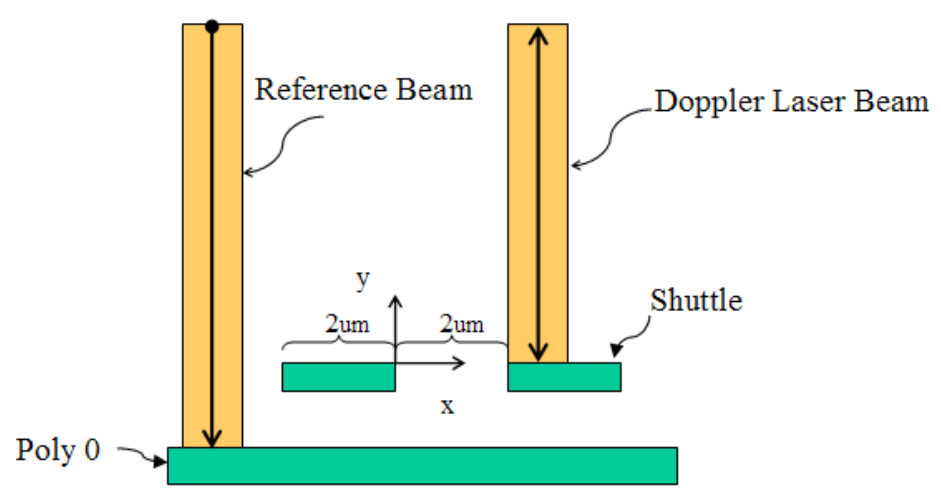

(a)

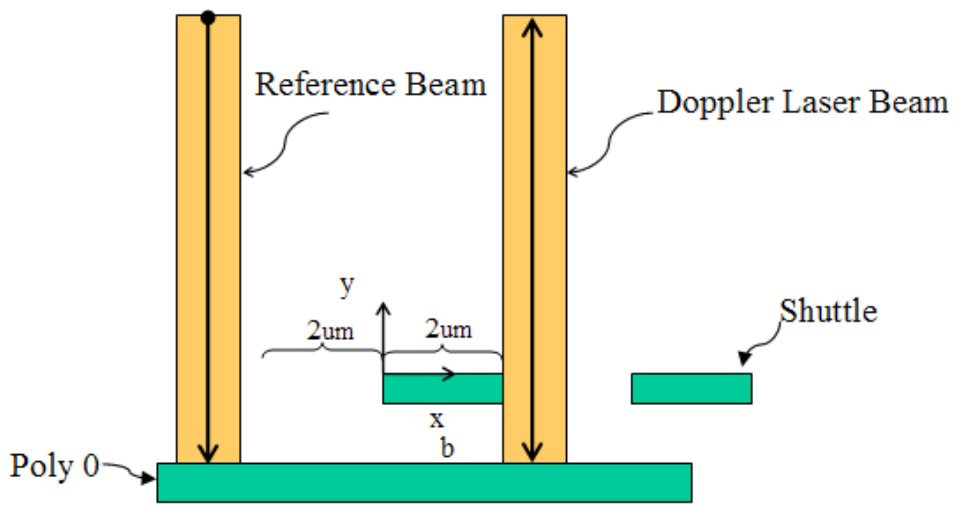

(b)

Fig. 2.3.3.1.1. Displacement feedback system using Doppler laser for optical displacement monitoring (side view).

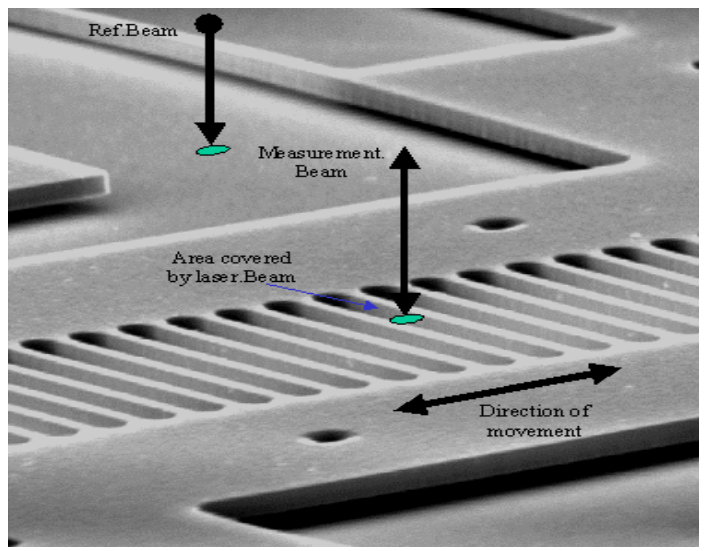

Fig. 2.3.3.1.2. Openings on the moving stage (Top View).

As mentioned earlier, the advantage of this method is a high Signal to Noise Ratio (SNR) compared to the traditional through-wafer monitoring technique and an easy laser beam placement on the device as shown in Figure 2.3.3.1.2. Interested readers in data recovery technique and instantaneous displacement monitoring are referred to (Dawson, Chen, Brown, Famouri \& Hornak. 2000), (Dawson. 2002), and (Izadian, Wang, Dawson, Hornak and Famouri. 
2007).

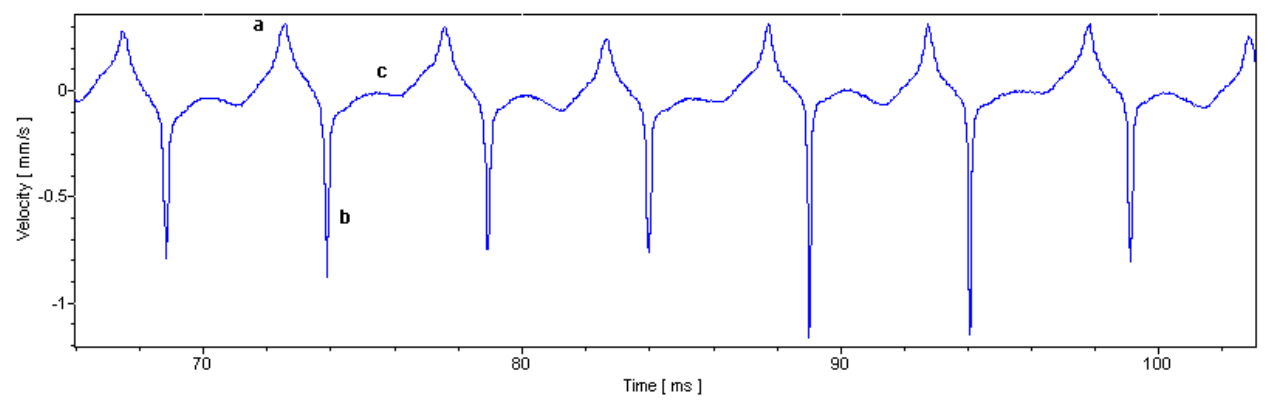

Fig. 2.3.3.1.3, Optical data recorded by the Doppler laser under displacement equal four microns. 


\section{Chapter 3,}

\section{Automatic Control of MEMS Devices}

The delicate structure of Micro Electro Mechanical Systems (MEMS) limits their reliability in cases of fault occurrence. Control systems can enhance their reliability of operation by providing fault-robust systems. This chapter illustrates a method for the safe operation of Lateral Comb Resonators (LCR) under fault conditions. Different types of fault are fabricated at chip level and experimentally tested in order to demonstrate the effectiveness of the reliability enhancement of operation in LCRs. Mass change and folded spring structural defects influence the device's behavior and reduce its reliability of operation. Here, Model Reference Adaptive Controllers are effectively used to enhance the reliability of faulty devices. Reliable operation of a $+5 \%$ additional mass considering environmental conditions and asymmetries on the device, demand almost 80\% higher control effort whereas $+20 \%$ in case of broken trusses or $+46 \%$ in case of adhesion point fault on a beam of folded springs. The simulation and experiment results demonstrate successful application of MRAC for reliable operation of LCRs by providing a fault-robust system.

In the second part of this chapter theoretical symmetric structure of MEMS devices are investigated. Electrostatic force is effective only along the axis of displacement and is neutralized elsewhere. This force is not a function of the distance between the stationary and the moving (shuttle) plates in the direction of the movement. However, manufacturing imperfections and fault conditions make the device operate asymmetrically and cause rotation of the shuttle structure in a lateral gap-closing direction, putting the device in an unstable region of operation (pull-in voltage limit). In this region, the rotated structure experiences an extra force which pulls the device into the region of instability beyond the pull-in voltage limits. This chapter illustrates the application of variable structure controllers to correct such behavior and to compensate for the uncertainties that exist in the microsystem parameters. The controller is equipped with a selftuning parameter estimation block and is designed to control the Lateral Comb Resonators under both normal and fault conditions. The controller utilizes through-wafer optical monitoring to 
provide the position feedback signal. The controller is implemented in a real-time control board and is experimentally verified to demonstrate the effectiveness of the control method under different operating conditions.

\subsection{Reliability Enhancement}

\subsubsection{Introduction}

Increasing complexity and integration level of MEMS devices demand high-level controllers in order to achieve the desired performance in their applications. The high uncertainties of their parameters caused by manufacturing processes of MEMS also dictate the need for sophisticated controllers. To date, development of control schemes for MEMS is behind compared to their fabrication and structure design (Bryzek \& Abbot. 2004). There is an enormous effort currently underway to mathematically model MEMS devices under different operating conditions and fault occurrence to identify their dynamics and enhance their reliability (Kolpekwar, Kellen \& Balnton. 1999), (Kolpekwar, Blanton. 1997), (Schanwald, Schwank, Sneigowski, Walsh, Smith, Petterson, Shaneyfelt, Winkur, Smith \& Doyle. 1998), (Rosing, Richardson, Dorey \& Peyton. 1999), (Brown \& Jansen. -), (Samper \&Trigg. 2003), (Tsuchiya, Tabata, Sakata \& Taga. 1997), (Srikar \& Senturia. 2002), (Li \& Thompson. 2005). Reliability enhancement techniques mostly address the structure reinforcement in thin film depositions which are being evaluated under different conditions such as shock. The control techniques, however, can enhance the reliability and elevate the system's robustness with the existing structures under severe conditions and sometimespermanent deformations such as fault.

Lateral comb resonators, used in many applications such as gyroscopes and prime movers of microsystems, are of particular interest in this section. Manufacturing steps and fault in microsystems initiate asymmetries and degrade the performance of the device.

Environmental conditions such as humidity and air pressure for those devices which operate in the air or for those which lose vacuum conditions, initiate air squeeze film and change the dynamics of the device. Temperature and storage conditions also affect the system's operation over time (Park, Horowitz \& Tan. 2001), (Wu, Zhe, Wang, Cheng, Modi \& Farmer. - ), (Reddy \& Jessing. 2004), (Rogers, Phinney. 2001), (Judy. 2001), (Gad-el-Hak. 2001). Fault in microsystems can be modeled as local defects, parameter tolerance, design problems, operation and/or system level faults (Schanwald, Schwank, Sneigowski, Walsh, Smith, Petterson, Shaneyfelt, Winkur, Smith \& Doyle. 1998). Fault can also be categorized as follows:

- Fault as change in structure of the device

- Fault resulting from environnemental contamination (i.e. pollution)

- Fault as change in operating environment 
Structural changes can cause permanent deformation in the geometry of the device; for example, short circuits may joint the parts of the device together. Deformation in the device's geometry may also be caused by broken parts in the device such as broken suspension springs or fingers (in the lateral comb resonator). In any of these cases, the resultant asymmetry changes the behavior of the system and causes a deviation from the desired output, which can be categorized as fault. For open-air applications of MEMS comb resonators or those with faulty package containers, the weight and size of the particles in their surroundings might limit the movement of the device and cause a fault in the structure of the microsystem. For instance, small particles can fall underneath the suspension springs or moving plates and act like an anchor and, as a result, change the spring constant values, limit the mobility of the device, and cause asymmetries. If a considerably heavy particle falls on the shuttle of the device, it might be observed as a mass change in the microsystem and change the behavior of the device and may therefore be considered as fault. Other types of behavioral change might occur because of changes in the operating environment of the microsystem. Change in the viscosity, temperature, pressure, or humidity of the surrounding material causes stiction and electromagnetic radiation and might result in behavioral change and consequently be recognized as fault. The fault in the microsystem can be modeled as changes of the mass, spring constant and/or damping coefficient. Much research has addressed the control of uncertain parameter systems such as MEMS devices. In this regard, open-loop controllers as well as closed-loop controllers (i.e. conventional PID) are designed according to the known parameters of the microsystem (Izadian, Hornak \&Famouri. 2006), (Garcia, et. al .1995), (Wang, Dawson, Hornak, Famouri \& Ghaffarian. 2004), (Dawson, Wang, Chen, Famouri \& Hornak. 2000). Advanced controllers make use of offline and online system identification techniques (Park, Horowitz \& Tan. 2001), (Izadian, Hornak \&Famouri. 2006), (Garcia, et. al .1995), (Wang, Dawson, Hornak, Famouri \& Ghaffarian. 2004), (Dawson, Wang, Chen, Famouri \& Hornak. 2000), (Wang, Dawson, Chen, Famouri \& Hornak. 2000), (Chen. Famouri \& Hornak. 1999), (Chueung, Horowitz, Howe. 1995), (Khamesse, Kato \& Nakamaura. 2003), (Izadian, Hornak \& Famouri. 2007), (Park \& Horowitz. 2003), (Shkel, Horowitz \& Seshia, Park \& Howe. 1999), (Leland. 2006). In most of these cases, the capacitive variation is the basis of displacement measuring techniques (Garcia, et. al .1995), (Chueung, Horowitz, Howe. 1995), (Park \& Horowitz. 2003), (Shkel, Horowitz \& Seshia, Park \& Howe. 1999).

This section of the chapter utilizes Model Reference Adaptive Controllers to enhance the reliability and robustness of Lateral Comb Resonators under severe parameter variations such as fault without reinforcement of the structure. The effect of fault on the folded spring configuration and mass content of the device is modeled and fabricated in different forms to be experimentally tested in trajectory tracking control. The displacement along the $\mathrm{x}$-axis is recorded by optical monitoring means and the difference between the recorded and the desired trajectory is used to update the controller gains. Three types of fault are considered for the experiment: 1). A five 
percent additional mass built on the shuttle of the device, 2). A point adhesion fault on the beam of the folded suspension spring, and 3). Broken trusses on one side of the folded suspension springs. Real-time control board dSPACE $®$ is used to implement the controller and test the reliable operation of LCRs during fault. Reliable trajectory control of these devices is therefore simulated and experimentally verified.

\subsubsection{Controller Design}

As illustrated in chapter 1, LCR devices contain uncertain parameters, and fault occurrence dominates their parameter variation. Thus, adaptive controllers can be applied to enhance the reliability level of microsystem. Consider two expressions for the system and its model, in which the model contains known values for the mass, spring constant and damping coefficient as the one expressed in (1.3.1), and the system contains values different from those of the model and is expressed in (1.3.8). The structures of the model and system are considered the same and expressed by second order differential equations. The model is written as

$$
\begin{aligned}
& \left\{\begin{array}{l}
\dot{x}_{1}=x_{2} \\
\dot{x}_{2}=\frac{1}{m}\left(-2 k_{s} x_{1}-\beta x_{2}\right)+\frac{F_{e}-F_{d}}{m} .
\end{array}\right. \\
& y=\left[\begin{array}{ll}
1 & 0
\end{array}\right]\left[\begin{array}{l}
x_{1} \\
x_{2}
\end{array}\right]
\end{aligned}
$$

Defining new variables $a_{m 1}=\frac{\beta}{m}, a_{m 2}=\frac{2 k_{s}}{m}$ and $k_{m}=\frac{1}{m}$, the model's $\operatorname{transfer}$ function $G_{m}(P)$ of (3.1.2.1), can then be written

$$
G_{m}(P)=\frac{y_{m}(p)}{r(p)}=\frac{k_{m}}{p^{2}+a_{m 1} p+a_{m 2}},
$$

where $a_{m 1}, a_{m 2}$ are the model's desired coefficients, $k_{m}$ is model's gain. $r(t)$ is the input signal to the model, $y_{m}(t)$ the resultant output signal, and $p$ the Laplace operator. The plant has the same order as that of the model with uncertain parameters in a non-Strictly Positive Real (non-SPR) format as the following transfer function

$$
G_{p}(p)=\frac{y_{p}(p)}{u(p)}=\frac{k_{p}}{p^{2}+a_{p 1} p+a_{p 2}} .
$$


The plant's transfer function $G_{p}(p)$ contains unknown and uncertain parameter values such as $a_{p 1}=\frac{\hat{\beta}}{\hat{m}}, a_{p 2}=\frac{2 \hat{k}_{s}}{\hat{m}}, k_{p}=\frac{1}{\hat{m}}$ with output $y_{p}(t)$ and control input $u(t)$ signals. $k^{*}$ is defined as the gain ratio of the model and the plant as

$$
k^{*}=\frac{k_{m}}{k_{p}}=\frac{\hat{m}}{m} .
$$

\subsubsection{Controller Design Procedure}

The structure of MRAC is explained in details in (Slotine \& Li. 1991) and (Ioannou \& Fian. 2006), but for the convenience of the readers, the design procedure is illustrated in simple steps as follows:

Step 1: A control law with constant coefficients, shown by $\left({ }^{*}\right)$, to be designed, is considered for the system as

$$
u^{*}(t)=k^{*} r+\theta_{1}^{*} \omega_{1}+\theta_{2}^{*} \omega_{2}+\theta_{0}^{*} y_{p}
$$

where $r$ is the input signal, $\omega_{1}$ and $\omega_{2}$ are filtered signals of the input and output of the plant respectively. The coefficients of $\theta_{1}$ and $\theta_{2}, \theta_{0}$ are control parameters. Since the plant parameters are unknown, we consider an estimate of the control coefficients at time $t$ to form the control command as

$$
u(t)=k(t) r+\theta_{1}(t) \omega_{1}+\theta_{2}(t) \omega_{2}+\theta_{0}(t) y_{p}
$$

The control command $u(t)$ is the applied voltage to the shuttle and based on (1.3.1), the required force is generated for the model (3.1.2.1). (Please see Fig. 3.1.2.1.1).

Step 2: in a system of order $n_{1}$, the control coefficient estimates and filtered signals are designed as

$$
\begin{aligned}
\theta(t) & =\left[\begin{array}{llll}
k(t) & \theta_{1}(t) & \theta_{2}(t) & \theta_{0}(t)
\end{array}\right]^{T}, \\
\omega(t) & =\left[\begin{array}{llll}
r(t) & \omega_{1}(t) & \omega_{2}(t) & y_{p}(t)
\end{array}\right]^{T},
\end{aligned}
$$


where $\theta$ is a $2 n_{1} \times 1$ vector of controller parameters, and $\omega$ is a $2 n_{1} \times 1$ vector of the corresponding filtered signals. Then the control command is written as

$$
u=\theta^{T}(t) \omega(t) .
$$

Step 3: The signals $\omega_{1}, \omega_{2}$ are $\left(n_{1}-1\right) \times 1$ vectors which satisfy

$$
\begin{aligned}
& \dot{\omega}_{1}=\Lambda \omega_{1}+H u \\
& \dot{\omega}_{2}=\Lambda \omega_{2}+H y
\end{aligned} .
$$

where $u, y$ are the input signals for $\omega_{1}, \omega_{2}$ filters respectively and $H$ is a $\left(n_{1}-1\right) \times\left(n_{1}-1\right)$ matrix such that $(\Lambda, H)$ is controllable.

Step 4: The estimation error is defined as the difference between the actual value and its estimates as

$$
\theta(t)=\theta^{*}+\phi(t)
$$

where $\phi(t)$ is the estimation error. Tracking error is defined as the error between the output of the plant and that of the model. It is expressed as

$$
e=G_{m}(p) \frac{\phi^{T}(t) \omega(t)}{k^{*}}
$$

Step 5: The controller parameters are updated according to the following adaptation technique

$$
\dot{\theta}=-\operatorname{sgn}\left(k_{p}\right) \gamma e(t) \omega(t),
$$

where $\gamma$ is a positive number and is called the adaptation gain. It should be noted that the sign of $k^{*}$ in (3.1.2.11) is the same as that of $k_{p}$.

Step 6: in a non-SPR system (see appendix A), the augmented error technique is used. This method is based on the addition of an augmentation signal $\eta(t)$ with an adaptive coefficient $\alpha(t)$ to the tracking error $e(t)$ which is expressed as

$$
\mathcal{E}(t)=e(t)+\alpha(t) \eta(t)
$$


where $\eta(t)$ is defined as

$$
\eta(t)=\phi^{T} G_{m}(p) \omega-G_{m}(p) \phi^{T} \omega
$$

Considering $\varpi=G_{m}(p) \omega$, the augmentation error gain adjusting technique obeys conditions

$$
\begin{gathered}
\dot{\theta}=-\frac{\operatorname{sgn}\left(k_{p}\right) \gamma \mathcal{E} \varpi}{1+\varpi^{T} \varpi}, \\
\dot{\alpha}=-\frac{\gamma \varepsilon \eta}{1+\varpi^{T} \varpi} .
\end{gathered}
$$

Figure 2.2.3.1 shows the controller structure of the model reference adaptive for systems with a relative degree one, and Figure 3.1.2.1.1 shows the augmentation technique in model reference adaptive control of an LCR system.

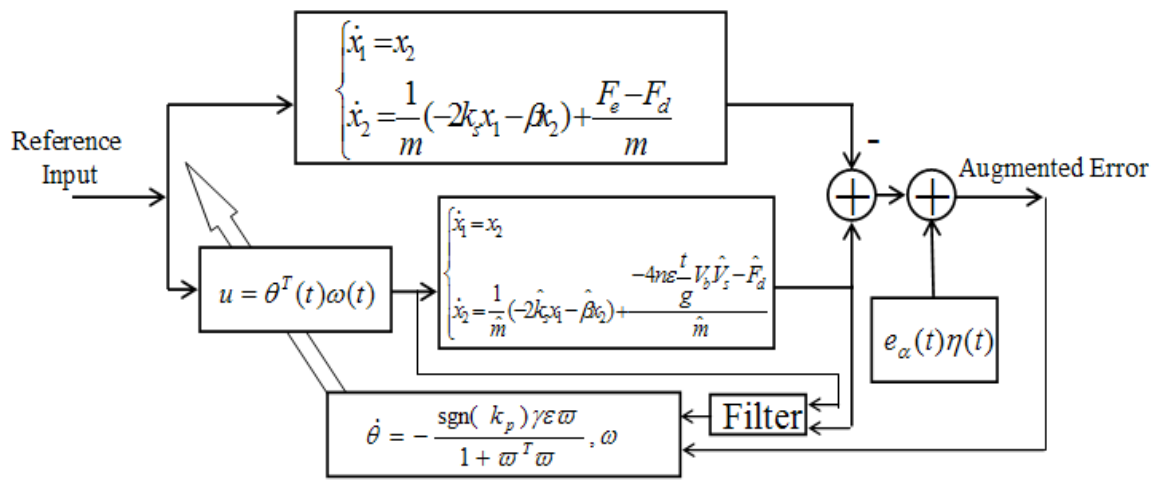

Fig. 3.1.2.1.1. Model Reference Adaptive Control for non-SPR systems, adaptation technique and error augmentation technique.

Proof of Stability: The stability of this controller can be found in several references such as (Slotine \& Li. 1991) and (Ioannou \& Fian. 2006).

\subsubsection{Reliability Evaluation: Simulation and Experimental Results}

\subsubsection{Simulation Results}

Simulations of designed controllers under mass, spring constant and damping uncertainties are investigated in this section. Different types of uncertainties act differently on the output and impose different control effort on the controller. Reliable operation of LCRs under fault 
conditions suggests that a control command be applied to the device. In an ideal case, the model contains the following values:

$m=2.2090 e-10(\mathrm{~kg}), \beta=7.09 e-7, k_{s}=0.0269$.

\subsection{Uncertainties on Mass of the Device}

Uncertainties on mass of the shuttle arise from several imperfect manufacturing steps. For test purposes, we have fabricated an additional $5 \%$ mass change on the shuttle of the device. For simulation and demonstration of fault tolerant controllers, we consider more mass change and increase this value in order to determine the allowable upper limit of these variations for a reliable operation. Figure 3.1.3.1.1 shows the tracking performance of a case with 10-times mass variation. Fast adaptation and zero error achievement are shown in Figure 3.1.3.1.2. As shown in Figure 3.1.3.1.3, the controller effort is very little in the case of mass change unless there exist another source of uncertainty in the system such as aging or stiction. In the experiment results section, we will explore these effects on the microsystem.

\subsection{Uncertainties on Damping Coefficient}

Operating conditions and surrounding material cause damping coefficient variations. Underdamped systems usually show longer movements and slower oscillation decay. In the case of a change in damping coefficient, a device that is simulated with as high as five times the actual damping of the system, MRAC can still provide near to zero tracking error at an acceptable control effort to compensate for the variations. The tracking profile in this case is shown in Figure 3.1.3.1.4. Tracking error reaches zero rapidly as the adaptation is completed. As it is seen, damping coefficient uncertainties are modeled as phase shift in the output of the actual system. The controller does not require very much effort to compensate for the phase shift.

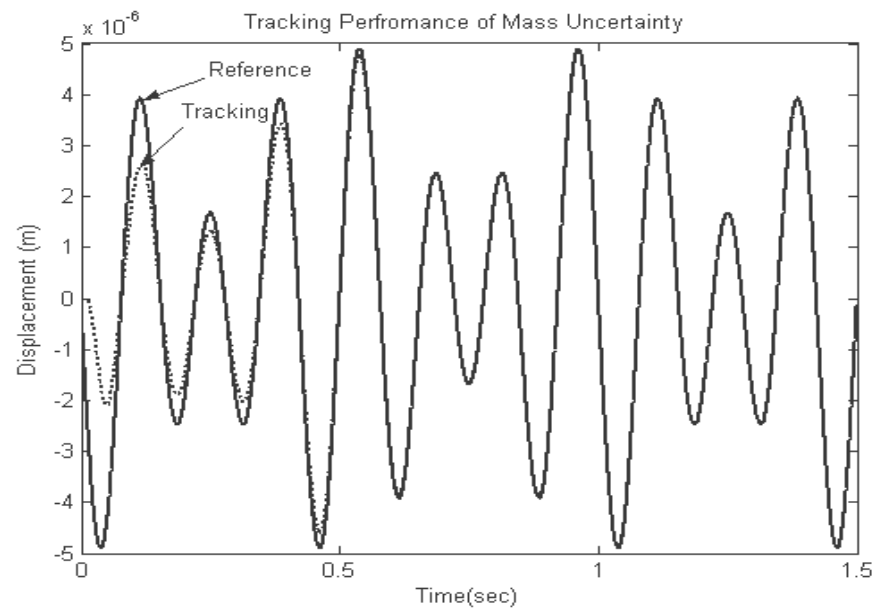

Fig. 3.1.3.1.1. Mass uncertainty effect modeling and tracking performance of MRAC in case of mass overestimation 


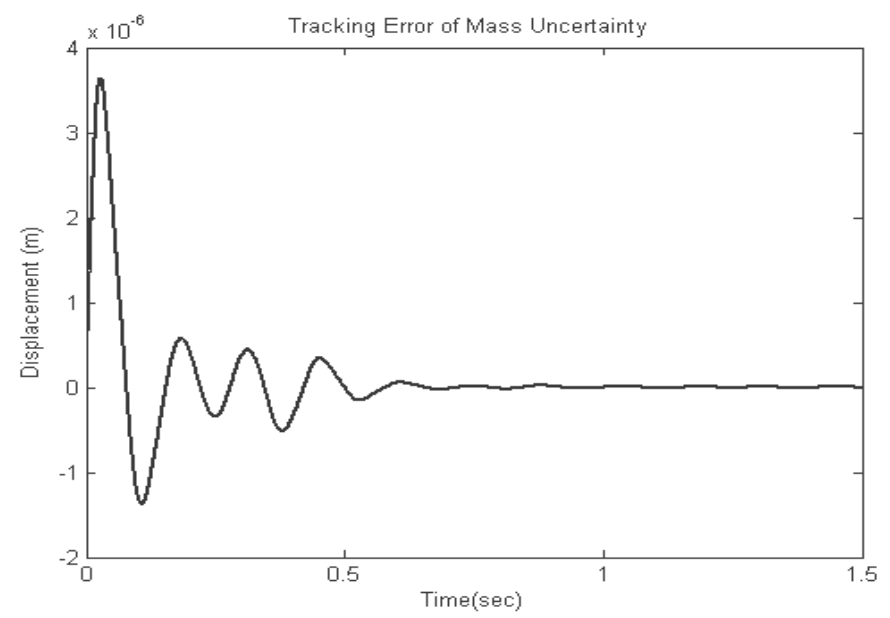

Fig. 3.1.3.1.2. Tracking error profile of MRAC in mass uncertainty case

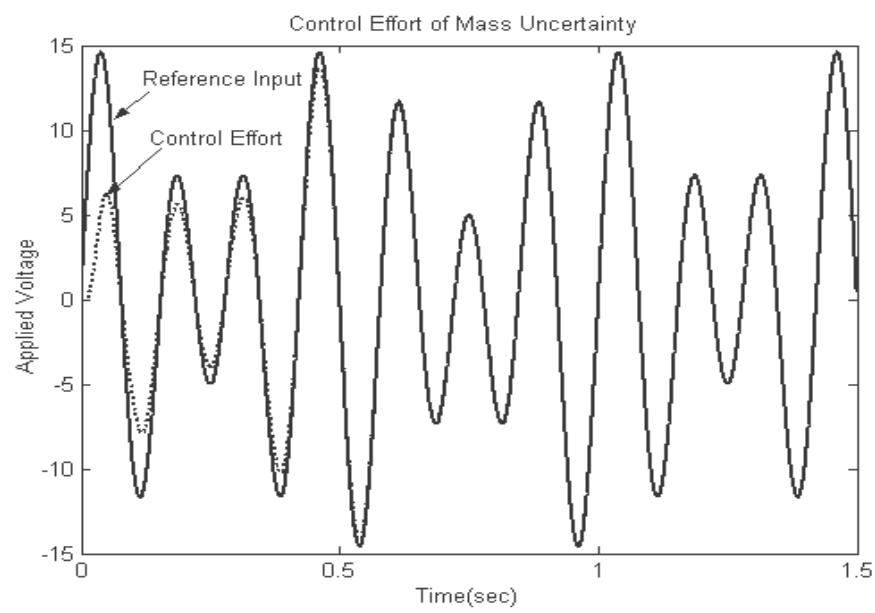

Fig. 3.1.3.1.3. Modeling the effect of mass uncertainties on control command generated by MRAC.

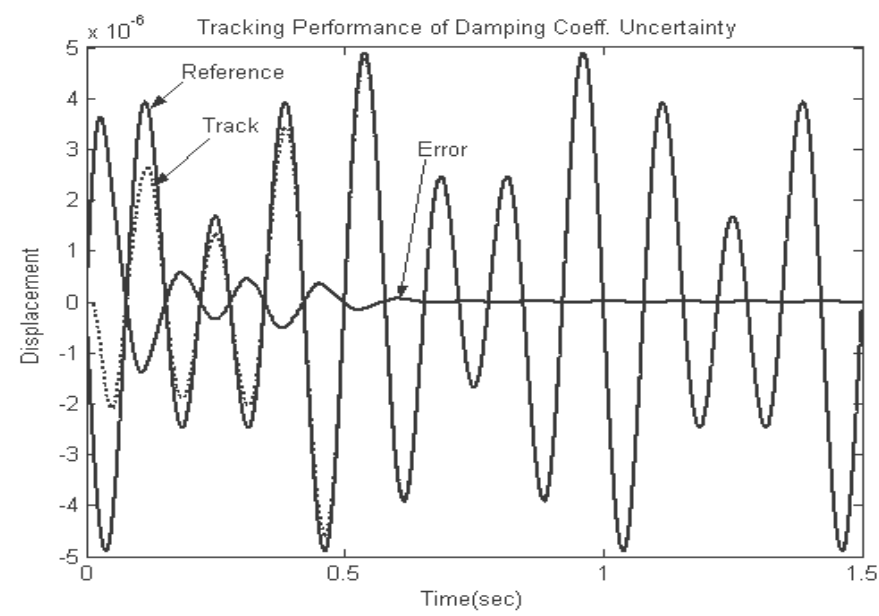

Fig. 3.1.3.1.4. Damping coefficient uncertainty modeling, performance of the tracking control, and tracking error profile are shown. 


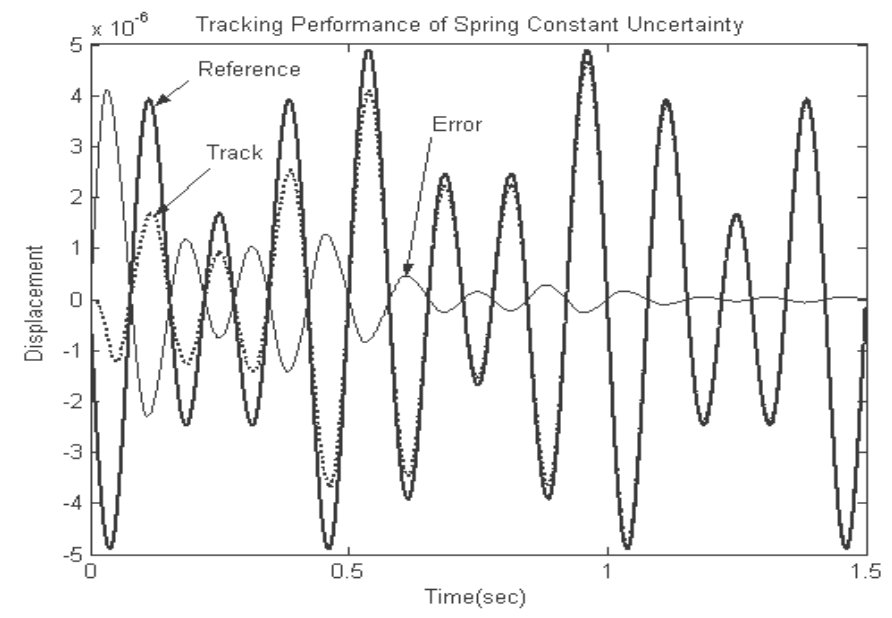

Fig. 3.1.3.1.5. Spring constant uncertainty modeling, tracking control performance and tracking error signal of Lateral Comb Resonator.

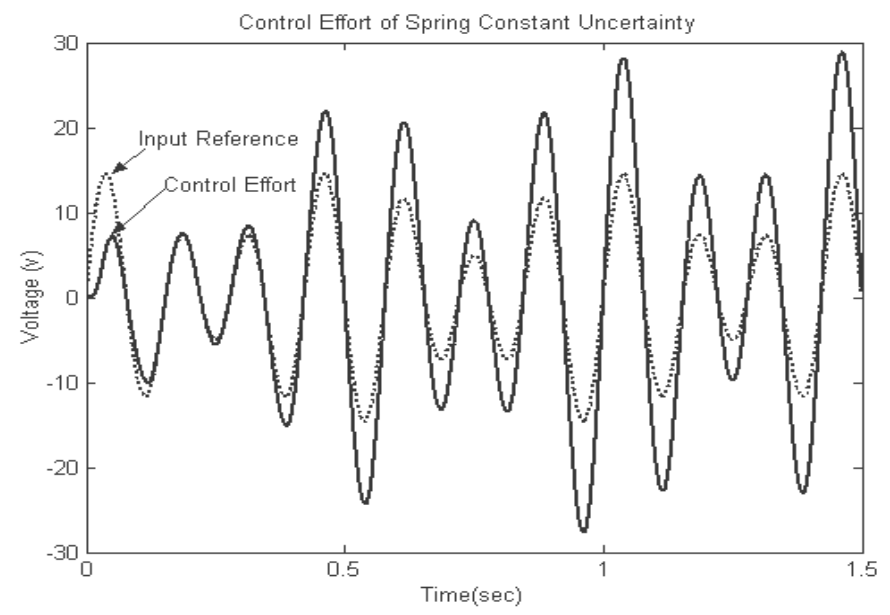

Fig. 3.1.3.1.6. Modeling the effect of spring constant uncertainty on control command generated by MRAC.

\subsection{Uncertainties on Spring Constant}

Spring constant variations result in significant behavioral changes in the device. Symmetric displacement is mainly influenced by folded spring structure. Resonant frequency is also shifted by variations of spring constant values. Figure 3.1.3.1.5 shows the tracking performance of the device with a spring constant twice the desired value. As Figure 3.1.3.1.5 shows, tracking error rapidly reaches zero as time increases. Figure 3.1.3.1.6 shows higher control efforts to compensate for the spring constant uncertainties in order to achieve a reliable operation in this case.

\subsubsection{Experimental Results}

The experiments were carried out on three different fabricated devices as described as follows: 
Three different devices, each with a different type of fault, were fabricated in order to test the reliability of operation of MEMS under fault conditions. Each of these cases was individually investigated using dSPACE ${ }^{\circledR}$, a real-time control board. Detailed design specifications of LCRs, their parameter values and dimensions can be found in (Konduparthi. 2005).

Case 1: A five-percent extra mass was fabricated on the shuttle of the device in order to represent the type of fault occurring in polluted environments and devices with variable loads. Reliable operation of LCRs under these conditions would expand their application in harsh environments. The effects of aging and environmental operating conditions were also taken into account by using an aged device to demonstrate reliable operation long after a manufacturing process that resulted in multiple uncertainties in the parameters. Reliable tracking performance of a desired trajectory under multiple-parameter variations is shown in Figure 3.1.3.2.1. In the case of multiple uncertainties in the device, the controller generated almost $80 \%$ stronger signals than the input-reference, achieving the reliable operation shown in Figure 3.1.3.2.2. Note that the aging limited the displacement and, as it is shown in Figure 3.1.3.2.3, over a period of 4 weeks it reduced the displacement of the device by $42.8 \%$.

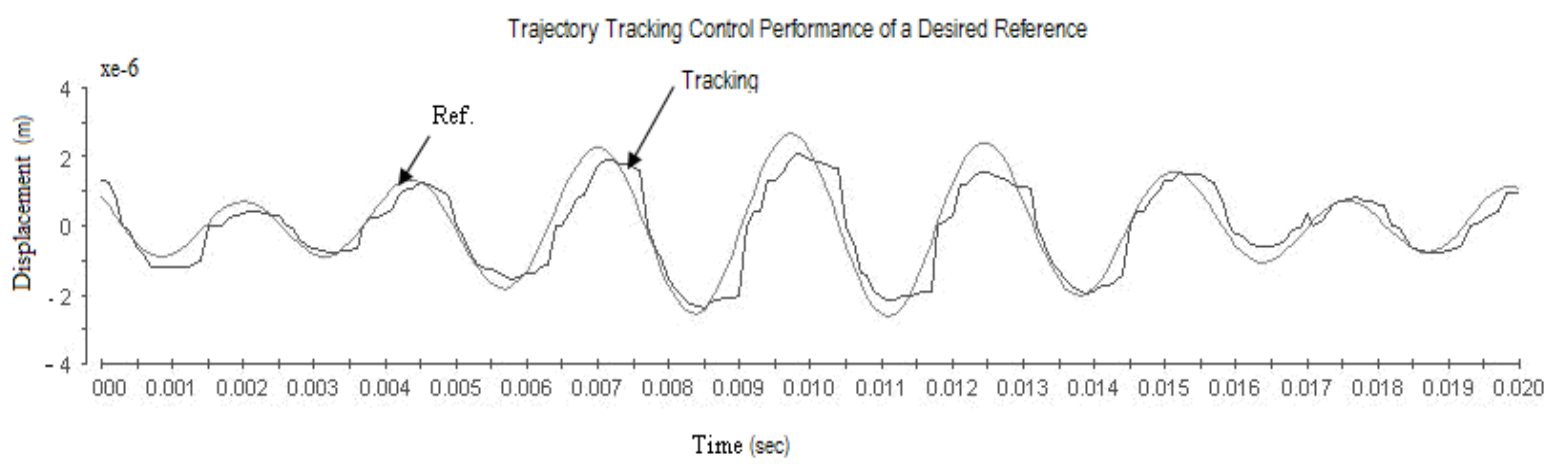

Fig. 3.1.3.2.1. Experiment results case one, tracking performance of a Lateral Comb Resonator with multiple uncertainties controlled by MRAC. 

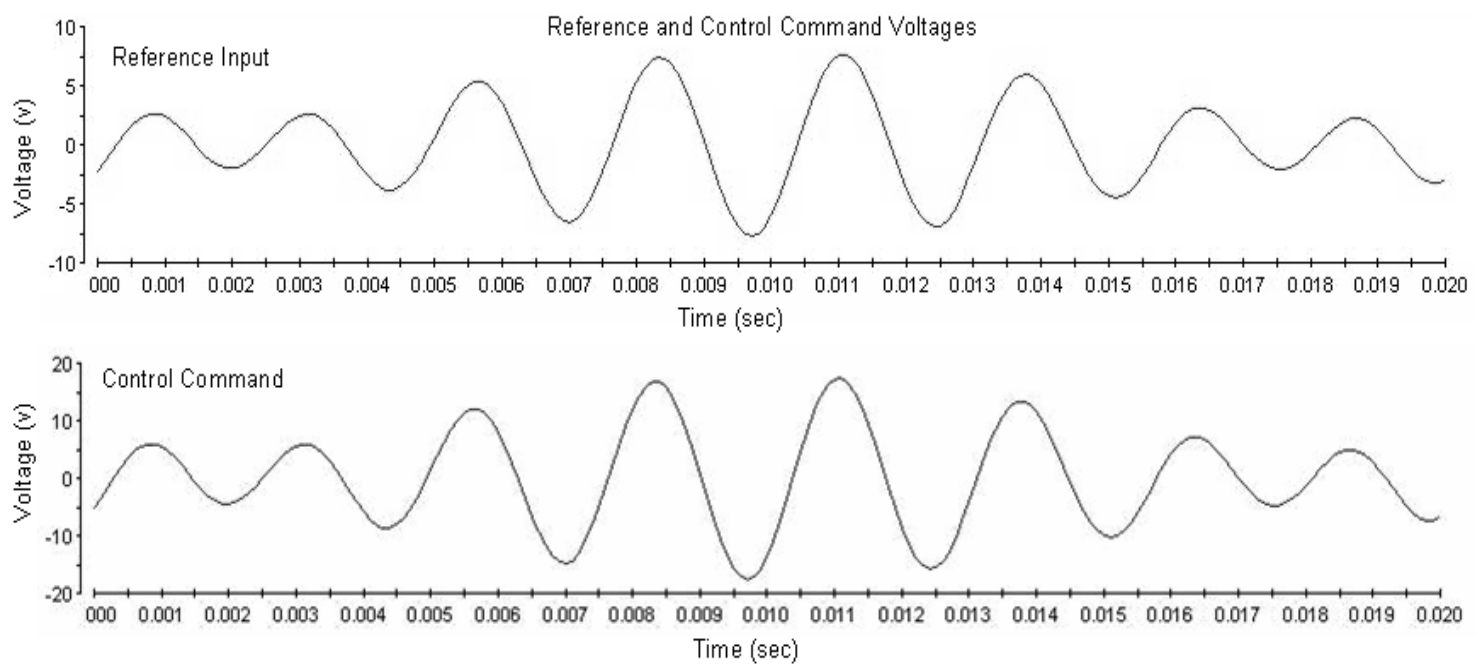

Fig. 3.1.3.2.2. Experiment results case one, a) Reference input signal, b) Control Effort; multiple uncertainties controlled by MRAC.

Case 2: Spring constant variations might occur during the fabrication process or presence of fault (fractures) and/or by random shocks entering to the structure of the device. Pollution and stiction might also result in spring constant variations. In this case, trusses of one side of the folded springs of a fabricated device, shown in Figure 3.1.3.2.4, were broken (thus changing the spring constant) in order to investigate reliable operation of LCR in the presence of asymmetries in suspension springs. Reliable tracking control of a desired trajectory under fault conditions is shown in Figure 3.1.3.2.5. In this case, the device experienced asymmetries in its displacement that increased the probability of an electric short circuit between the moving and stationary plates. In addition, the respective displacements of the two sides did not coincide (due to the difference in spring constant value), which resulted in the plates being placed non-uniformly along the original displacement path. The controller in this case generated about $20 \%$ higher efforts than the input-reference in Case 1, with almost 80\% higher values for reliable operation. The control effort is shown in Figure 3.1.3.2.6. Thus, In case of fault in the microsystem, the controller can successfully maintain reliable operation by providing a suitable control command. 


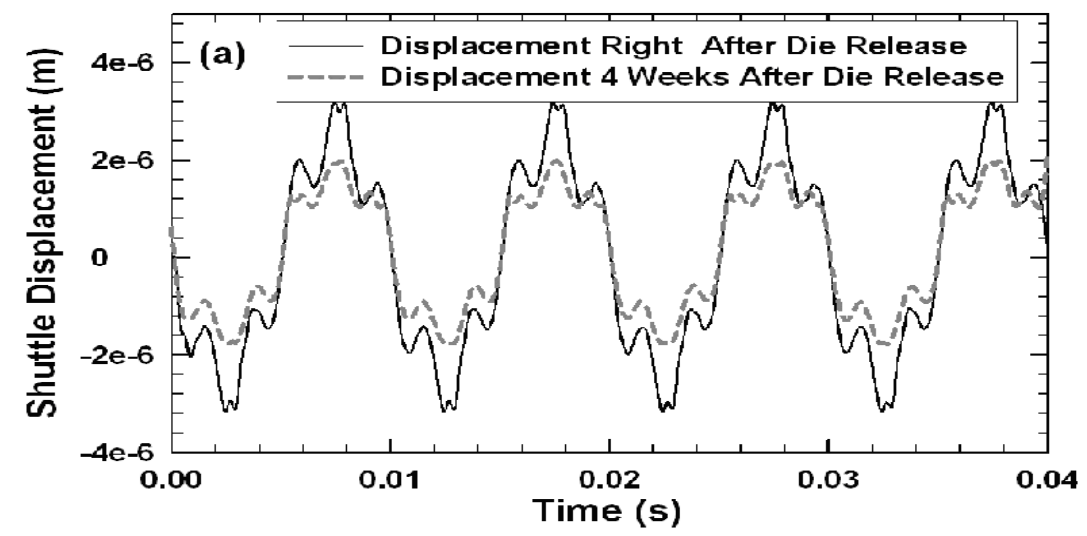

Fig. 3.1.3.2.3. The effect of aging on the displacement of Lateral Comb Resonator. Stiction and wearing on the device result in lower displacements. (Experimental results)

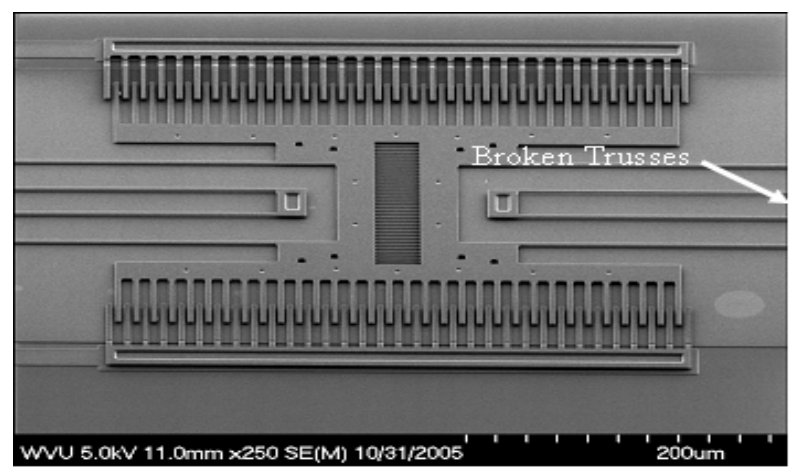

Fig. 3.1.3.2.4. The device with broken trusses and asymmetric displacement. Picture by WVU MEMS Research Group.

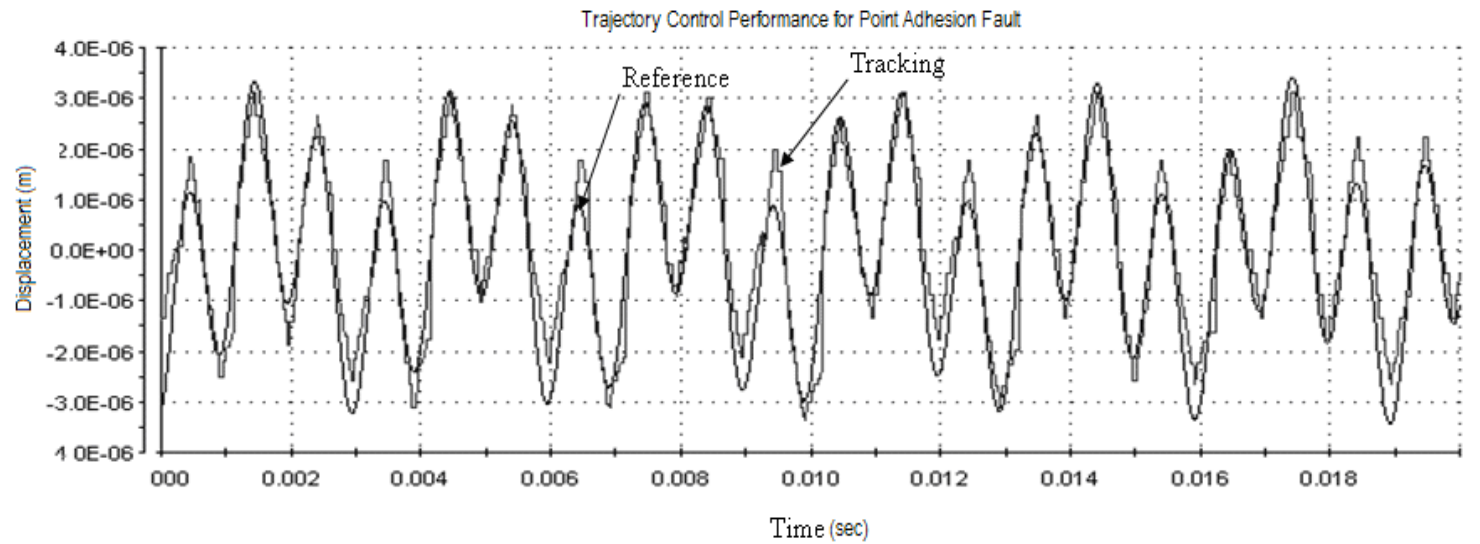

Fig. 3.1.3.2.5. The trajectory control of the broken trusses device on the suspension spring beam, case two of the experiments. 


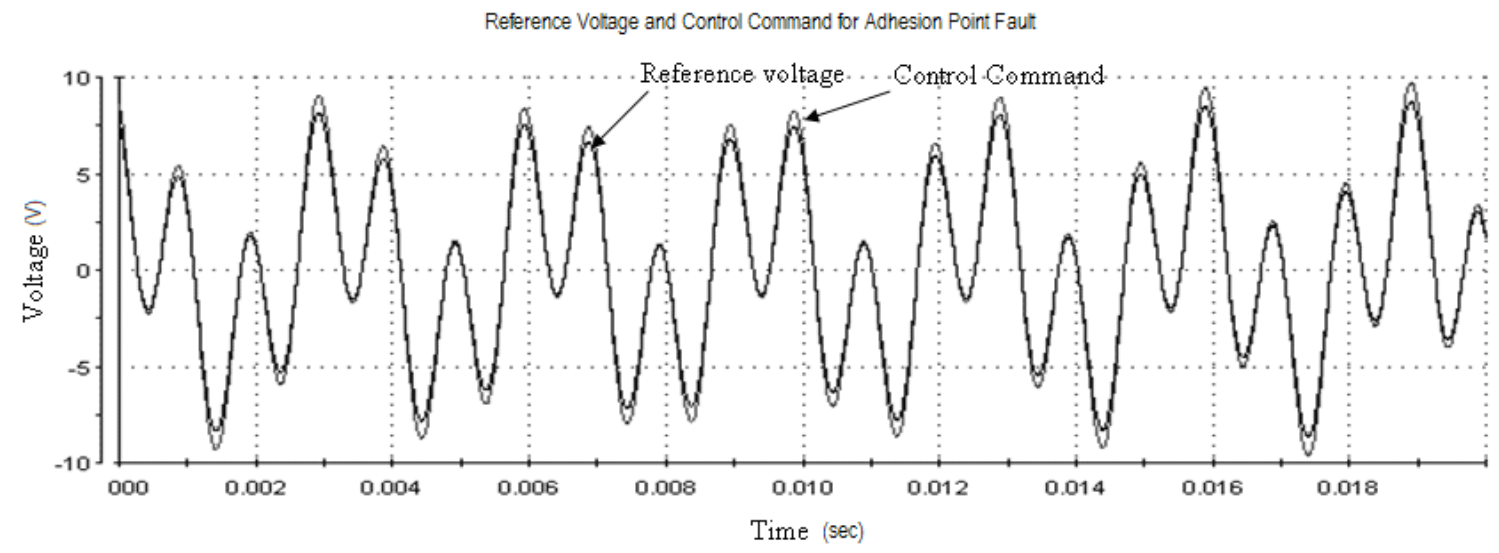

Fig. 3.1.3.2.6. The applied reference voltage and the Control Command of MRAC, case two of the experiments.

Case 3: Limitation of displacement can also occur by anchoring the folded springs on one side. By doing this, displacement is limited in one side due to shorter spring beams. It requires additional effort from the controller to have the device operate in a tracking mode. Reliable operation of the device is critical in this regard. To simulate this type of failure and demonstrate reliable operation with a controller, a point fault adhesion was fabricated on the beam of one side of the folded springs, shown in Figure 3.1.3.2.7. Applying the controller, reliable tracking performance of the device was achieved and shown in Figure 3.1.3.2.8. In this case, stiffer springs with higher spring constant values required $46 \%$ additional effort than the input-reference. The corresponding control command is shown in Figure 3.1.3.2.9. Tracking error in the recent cases due to the existence of noise in the system and higher relative degree than one reached to a limited value.

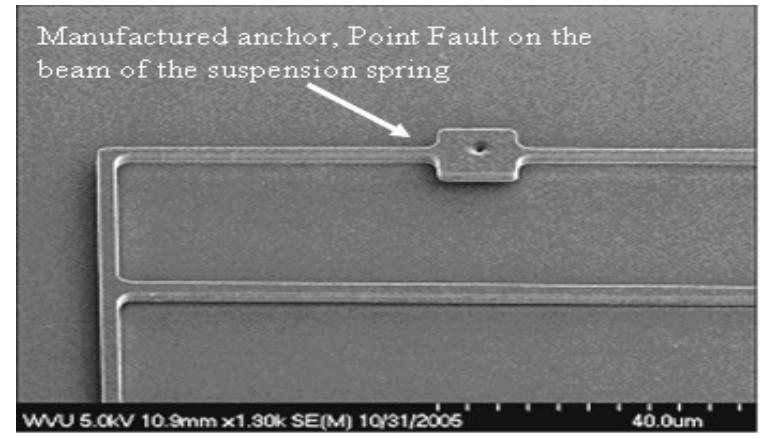

Fig. 3.1.3.2.7.. Point fault adhesion on the beam of the suspension spring. 


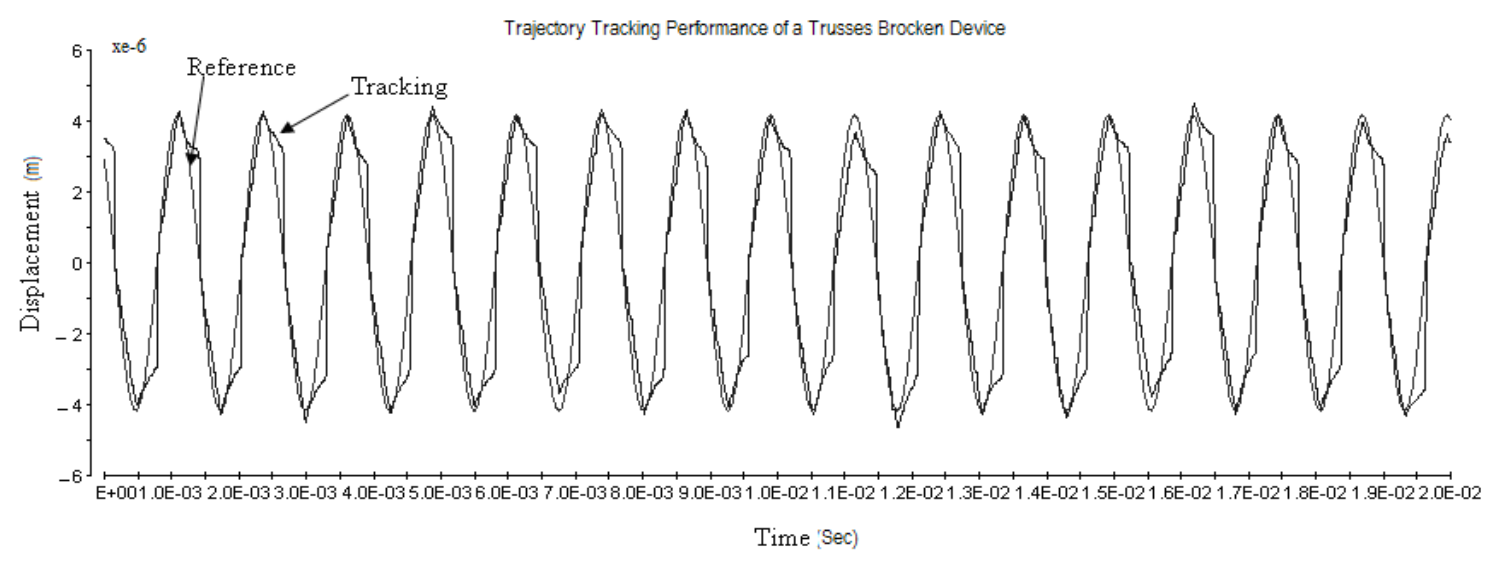

Fig. 3.1.3.2.8. Trajectory tracking control performance; Case of the point adhesion on folded springs.

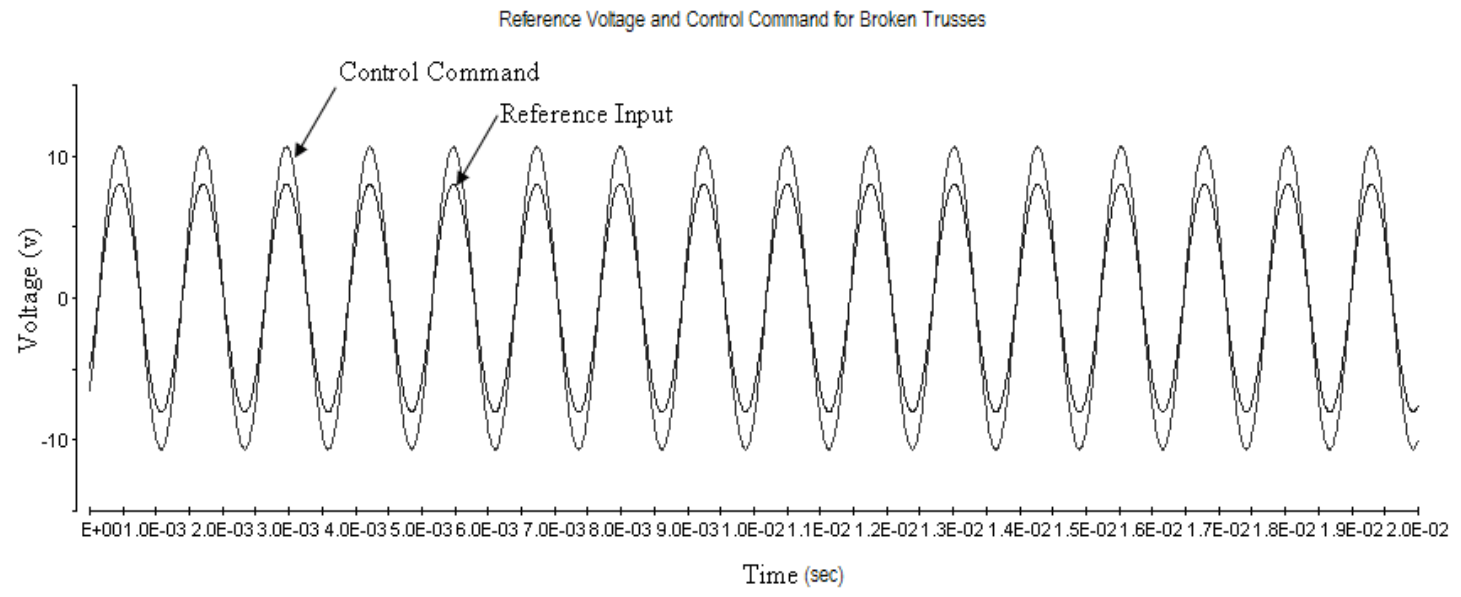

Fig. 3.1.3.2.9. The control effort and applied reference input to the system in case three of experiments. 


\subsection{Shuttle Rotation Control of MEMS Lateral Comb Resonators Under Fault}

\subsubsection{Introduction}

The structure of Lateral Comb Resonators (LCR) experiences rotation under conditions of overexcitation, over-loading and the occurrence of fault. Ideally, the equal distance of the shuttle fingers from the stationary comb fingers generates equal absorption at both sides for the shuttle finger and leaves the device stationary except at the main direction of movement. Therefore, the generated electrostatic force along the direction of movement becomes a function of the constant inter-digit side gaps of the comb drives. In reality, however, imperfect manufacturing steps and parameter variations can cause structural asymmetries and influence the motion of the device (Gal-el-Hak. 2002). The occurrence of fault can also influence the symmetrical motion and push the device toward instability.

The system's parameter values-such as the mass, spring constant and damping coefficientpossess uncertain variations among different devices of similar design and might change during the operation. Moreover, occurrence of a fault could result in asymmetries in the output displacement of the microsystem and make the shuttle rotate along the point of fault. Rotation of the shuttle makes the gap closing uneven and locates the shuttle fingers in a closer position to the stationary combs. This non-uniform gap closing would result in extra attraction force production in both the $x$-and $y$-axes and results in the pull-in voltage effect (Senturia. 2001) in the side-gaps of LCR fingers. The occurrence of fault and its resultant shuttle rotation are explained in detail in the problem definition section (3.2.2).

In some applications (such as gyroscope arrays), a number of LCRs with different parameters are required in order to be controlled to follow the same pattern and resonate coherently. In this case, there is a need for precise control of these devices to follow the desired trajectories regardless of their different parameter values and variations. Conventional controllers (Wang, Dawson, Hornak, Famouri \& Ghaffarian. 2004) can control the displacement, but they require the precise values of the system parameters (Chueung, Horowitz, \& Howe. 1995), (Chen, Famouri, \& Hornak. 1999). Therefore, higher-level controllers are required for systems with uncertain parameters (Dawson, Wang, Chen, Famouri \& Hornak. 2000), (Park, Horowitz, \& Tan. 2001), (Khamesse, Kato \& Nakamura. 2003).

Variable Structure Controllers (VSC) were developed in the early 1950s in the Soviet Union in order to stabilize the control systems (Utkin. 1992). Applying sliding mode theory to variable structure controls introduces the concept of sliding surfaces, which separates regions of operation into sliding and non-sliding parts. Switching surfaces determines the sign of the control command according to the sliding direction (Huna, Gao \& Hung. 1993). Generally, variable structure controllers are robust and perform well under parameter uncertainties and noise (Huna, Gao \& Hung. 1993), (Young. 1978). They also perform tracking well under transient conditions 
(Furuta, Kosuge \& Kobayashi. 1989), (Friedland. 1996). Self-tuning estimators can generate a precise and hypothetical model of Lateral Comb Resonator for variable structure controllers. A combination of the Self-Tuning and Variable Structure Controller (STVSC) provides an accurate model of the system and acts as a robust switching controller (Xu, Heng \& Wang. 1996). Furuta in (Furuta, Kosuge \& Kobayashi. 1989) has introduced the application of the variable structure controller to unknown parameter plants using minimum variance and least square techniques.

This section provides a second order model with uncertain parameters for LCRs and investigates the shuttle rotation and pull-in voltage effect due to fault. The fault is modeled as an adhesion point on the shin of the folded springs, and parameter uncertainty is modeled as a $5 \%$ additional mass on the shuttle of a separate device. These two devices are designed and fabricated to experimentally validate the effectiveness of Self-Tuning Variable Structure Controllers in controlling of LCRs under both normal and near pull-in voltage limit conditions.

\subsubsection{Problem Definition}

Occurrence of fault in the microsystem might change the symmetry of shuttle's displacement. For example, a particle attached underneath the suspension spring or a fracture on the flexure arm could limit the displacement on one side and result in rotation of the shuttle. To experimentally show the effect of the limiting point adhesion fault, a device with an anchor on the shin of the folded spring structure was fabricated (Figure 3.1.3.2.7). Displacement of the shuttle along the $\mathrm{x}$-axis results in rotation of the stage through angle $\alpha$ from its initial location along the point adhesion (Figure 3.2.2.1). This rotation produces an uneven gap closing along the y-axis, orthogonal to the direction of movement. This may change the force balance in that direction and result in more attraction in one side with respect to the other side of the comb fingers. Figure 3.2.2.2 shows the schematics of one inter-digit force balancing under normal and fault conditions. In the case of longer displacement, the shuttle comb fingers become closer in position to the stationary combs and make the system behave more like a set of parallel plate actuators in gap closing in the $y$-axis direction. This phenomenon produces an extra force along the rotated angle of the device by generating two force components on the $\mathrm{x}$ and $\mathrm{y}$-axes, both of which contribute to the shuttle rotation under unbalanced conditions. 


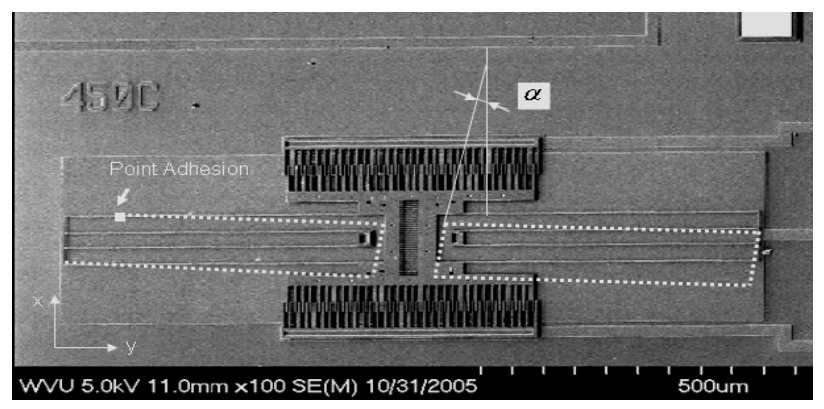

Fig. 3.2.2.1. Schematic demonstration of the rotation of the shuttle in long displacement in the presence of the point fault adhesion. (To better show the shuttle rotation, the amount of rotation is exaggerated).

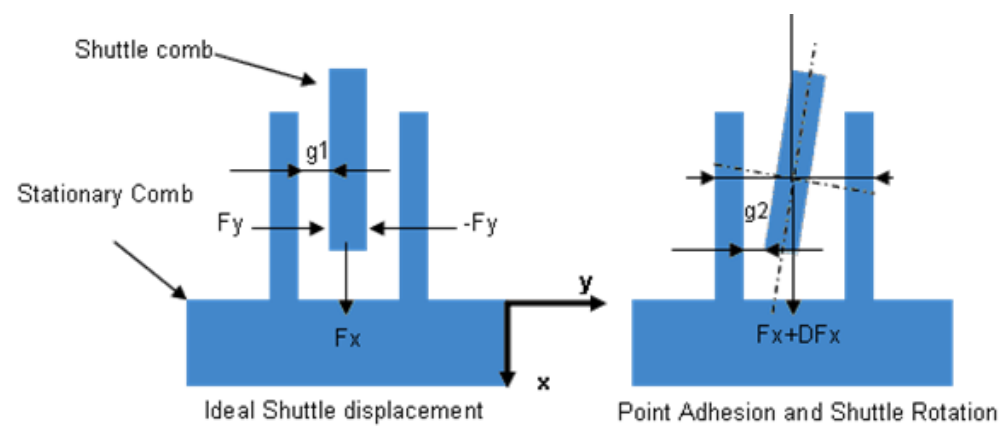

Fig. 3.2.2.2. Rotation of the shuttle and the force generation in $\mathrm{x}$ and $\mathrm{y}$-axes due to the adhesion point.

Similar to parallel plate actuators, if the gap $g_{2}$ is smaller than $2 / 3$ of the total gap $g_{1}$, the device becomes unstable. Then the force along the $\mathrm{x}$-axis becomes dependent on the distance of the plates $\left(\mathrm{g}_{2}\right)$ which at a certain point produces a pull-in voltage effect (Senturia. 2001). The rotation angle depends on the amount of displacement along the x-axis, where the natural positive feedback of the electrostatic force introduces more complexities to the control of the device. Under these conditions, STVSC is employed to mitigate the uncertain parameter variations and control the trajectories beyond the pull-in limits under fault conditions.

\subsubsection{Self-Tuning Estimator}

The self-tuning systems adjust the parameters of a hypothetical system and match the output of the hypothetical system with that of the actual system. Different parameter estimators are used depending on the characteristics of the system. In systems with highly varying dynamics or those with sub-dynamics, the gradient method is commonly used. As the sets of input-output change, the self-tuning estimator is updated in order to follow the zero estimation error. In this chapter, the gradient method is applied to follow the parameter variations from different devices and during the operation (fault).

Considering a model follower controller (Model based VS), the dynamics of the model (with known parameters) and that of the plant (with unknown parameters) in general $\mathrm{n}^{\text {th }}$-order systems 
are represented as

$$
\begin{aligned}
& \dot{y}_{p}=A_{p} y_{p}+B_{p} U_{p} \\
& \dot{y}_{m}=A_{m} y_{m}+B_{m} U_{m}
\end{aligned},
$$

where $y_{m}$ is the hypothesized model's output and $U_{m}$ is the model's input. $U_{p}$ and $y_{p}$ are the related input-output values applied to LCR (plant). $A_{p}, B_{P}$ are the plant system and input matrices respectively, and $A_{M}, B_{M}$ are the corresponding system and input matrices of the model. Filtering the input-output equation using an $\mathrm{n}^{\text {th }}$ order monic polynomial yields the matrix form system as (for mathematical derivations please see (Slotine \& Li. 1991))

$$
y=\theta \cdot w,
$$

where $\theta^{T}$ is a $2 n$ vector containing the unknown coefficients, and $w$ is a $2 n$ vector containing the filtered signals

$$
\begin{gathered}
\theta=\left[\left(\alpha_{0}-a_{0}\right)\left(\alpha_{1}-a_{1}\right) \ldots\left(\alpha_{n-1}-a_{n-1}\right) b_{0} \ldots b_{n-1}\right], \\
w=\left[\frac{y}{A_{0}} \frac{p y}{A_{0}} \ldots \frac{p^{n-1} y}{A_{0}} \frac{u}{A_{0}} \ldots \frac{p^{n-1} u}{A_{0}}\right]^{T},
\end{gathered}
$$

where $A_{0}$ is the aforementioned filtering polynomial with known coefficients $\left[\alpha_{0} \alpha_{1} \ldots \alpha_{n-1}\right]$ and $p$ is the Laplace operator. During the adaptation process, plant parameters are adjusted such that the estimation error reaches zero. Applying the estimated values $\hat{\theta}$, the estimated output is obtained as

$$
\hat{y}=\hat{\theta} w,
$$

The difference between the plant's output and that of the model is the estimation error $e_{1}$, and is computed as

$$
e_{1}=\hat{y}-y \text {. }
$$

The parameter adaptation technique depends on the estimation error, a corresponding filtered signal and the adaptation gain which determines the adaptation rates. It can be obtained as 
(Slotine \& Li. 1991)

$$
\dot{\hat{\theta}}=-P_{0} w e_{1},
$$

where $P_{0}>0$ is the adaptation gain. The self-tuning technique considering the parameter adaptation rule (3.2.3.7) is asymptotically stable; considering a Liapunov function as

$$
V=\tilde{\theta}^{T} \tilde{\theta}
$$

yields:

$$
\dot{V}<0 \text {. }
$$

Global stability of the self-tuning gradient estimator is therefore proven (Levine. 1996), where $\tilde{\theta}$ is the parameter estimation error. This assures the zero estimation error of the estimator and its convergence in a proper time, i.e.

$$
t \rightarrow \infty \Rightarrow e_{1} \rightarrow 0
$$

\subsubsection{Self-Tuning Variable Structure Controller}

In the previous section, the model estimating technique was introduced and explained. In this section, fundamentals of variable structure controllers are described and applied to the selftuning unit.

Variable Structure Controllers are high-speed feedback systems that switch between two values. The purpose of the switching is to drive systems (in this case: LCRs) with unknown parameters to follow a specified trajectory called the "switching surface," also known as the "sliding surface" (Levine. 1996). The first stage of designing a variable structure controller is to design a switching surface in which the LCR device can be controlled at stable and unstable equilibrium points. (Furuta,Young. 1978), (Furuta, Kosuge \& Kobayashi. 1996), (Furuta. 1991) have shown that a sliding sector can be applied instead of the sliding surface with a specific control law. The second stage is to design the switching control law and drive LCRs to follow a desired trajectory. In this regard, Liu Hsu et. al. (Hus \& Lizarralde. 1996) have introduced variable structure controllers without the need for derivatives of the corresponding signals. In the following section, the VSC's control law is discussed according to its application in Micro Electro Mechanical Systems.

\subsubsection{Switching Surface Design}

The existence conditions of the control law are presented in (Young. 1978) and (Hus \& 
Lizarralde. 1996). We assume that the conditions hold with the same matrices in equation (3.2.3.1). In the STVSC, the model is estimated and updated recursively until zero error conditions are obtained. The switching hypersurfaces, $s$, are hyperplanes (Young. 1978) if

$$
s=G e_{1}=0,
$$

where $G$ is the gain-switching matrix, and $s^{T}=\left(s_{1}, \ldots s_{n}\right)$ defines the sliding mode surfaces. The design objective is to find the gains of the controller and a gain-switching matrix in which the error reaches zero, i.e. (Young. 1978)

$$
\lim _{t \rightarrow \infty} e_{1}(t)=0
$$

However, since the self-tuning estimator assures a zero estimation error, the sliding surface exists after the adaptation is completed. These two criteria provide further proof for the existence of the control law.

\subsubsection{Control Law}

The objective of the control is to find out the control command $u$ in which the output of the system tracks the desired trajectory. In the meantime, the self-tuning estimates the unknown parameters $\hat{\theta}$ of the system. In equation (3.2.3.1), the pairs of $\left(A_{P}, B_{P}\right)$ are controllable and $A_{M}$ is a stable matrix. The VSC control law is described as (Young. 1978)

$$
U_{P}=\left[K_{p}\left|y_{P}\right|+K_{e}\left|\left(y_{M}-y_{P}\right)\right|+K_{M}\left|U_{M}\right|\right] \operatorname{sgn}(s),
$$

where $K_{p}, K_{e}$ and $K_{M}$ are the controller's coefficients, which are computed according to the sliding surface estimation error and sets of input-output values analyzed recursively, and $U_{m}$ is the input to the hypothetical model. The sliding surface is an interface between the adaptive estimation and the VSC. The gain-switching matrix and the controller parameters are computed based on the following equations: (Hsu \& Cheng. 1998)

$$
\begin{gathered}
K_{p}=\left(G B_{p}\right)^{-1} G A_{p}, \\
K_{e}=\left(G B_{p}\right)^{-1} G\left(A_{m}-A_{p}\right), \\
K_{m}=\left(G B_{p}\right)^{-1} G B_{m} .
\end{gathered}
$$


These equations describe the controller gains as functions of the gain-switching matrix and the system and model parameters. The estimation error is determined through the self-tuning algorithm and is used to form the sliding surface and control command. The design of the gainswitching matrix, which was introduced by Utkin and Yang (Utkin \& Yang. 1978), is explained in simplified steps as follows:

The general system (3.2.3.1) can be rewritten as

$$
\left[\begin{array}{l}
\dot{y}_{1} \\
\dot{y}_{2}
\end{array}\right]=\left[\begin{array}{ll}
A_{11} & A_{12} \\
A_{21} & A_{22}
\end{array}\right]\left[\begin{array}{l}
y_{1} \\
y_{2}
\end{array}\right]+\left[\begin{array}{l}
0 \\
B
\end{array}\right] u,
$$

where $A_{11}, A_{12}, A_{21}$, and $A_{22}$ are $(n-m) \times(n-m),(n-m) \times m, m \times(n-m)$ and $m \times m$ sub-matrices, respectively. Let $A^{\prime}=A_{11}, B^{\prime}=A_{12}$, then matrix $G_{11}$ is defined as

$$
G_{11}=B^{\prime T} P^{\prime}
$$

where $P^{\prime}$ is the solution of the following algebraic Ricatti equation

$$
P^{\prime} A^{\prime}+\left(A^{\prime}\right)^{T} P^{\prime}-P^{\prime} B^{\prime}\left(R^{\prime}\right)^{-1}\left(B^{\prime}\right)^{T} P^{\prime}+Q^{\prime}=0
$$

where $Q^{\prime}$ and $R^{\prime}$ are positive definite matrices, and the gain-switching matrix is defined as

$$
G=\left[G_{11} I\right] M_{1},
$$

in which $M_{1}$ is defined such that the following equation

$$
M_{1} B_{p}=\left[\begin{array}{l}
0 \\
B
\end{array}\right]
$$

holds with a non-singular $B$. 


\subsubsection{Formulation for MEMS Lateral Comb Resonator \\ 3.2.5.1 Self-Tuning Estimator}

The transfer function of a LCR in equation (1.3.1) can be written as

$$
y=\frac{k_{p} u}{p^{2}+a_{p 1} p+a_{p 2}},
$$

where unknown coefficients $a_{p 1}=\frac{\beta}{m}, a_{p 2}=\frac{2 k_{s}}{m}, k_{p}=\frac{1}{m}$ are to be identified during the online parameter estimation process; $m, \beta, k_{s}$ are unknown parameters of the system. The input-output matrix representation with the filtered signal vectors is (Slotine \& Li. 1991)

$$
y=\theta^{T} w(t),
$$

where $\theta$ is a $4 \times 1$ vector containing the unknown values and $w$ is a $4 \times 1$ vector containing the filtered input and output signals, identified by “*”, as

$$
\begin{aligned}
\theta & =\left[a_{p 2}^{*}, a_{p 1}^{*}, k_{p}^{*}, 0\right]^{T} \\
w & =\left[y^{*}, p y^{*}, u^{*}, p u^{*}\right]
\end{aligned}
$$

Therefore, the self-tuning estimator updates the system parameters as $k_{p}, a_{p 1}, a_{p 2}$. Accordingly, the parameter adaptation law can be written as

$$
\dot{\hat{\theta}}=-P_{0} w e_{1} .
$$

This identifies an estimate of $A_{p}, B_{p}$ as $\hat{A}_{p}, \hat{B}_{p}$ for the VS controller.

\subsubsection{Design of Control Law}

The state space expression of (3.2.5.1.1) can be written in a canonical form as 


$$
\left[\begin{array}{l}
\dot{y}_{1} \\
\dot{y}_{2}
\end{array}\right]=\left[\begin{array}{cc}
0 & 1 \\
\frac{-k}{m} & \frac{-\beta}{m}
\end{array}\right]\left[\begin{array}{l}
y_{1} \\
y_{2}
\end{array}\right]+\left[\begin{array}{l}
0 \\
1 \\
m
\end{array}\right] u \text {. }
$$

Therefore, comparing $(3.2 .5 .2 .1)$ with $(3.2 .4 .2 .5)$ yields $A^{\prime}=0, B^{\prime}=1$ and $M_{1}=I$, and substituting these values into the Ricatti equation (3.2.4.2.7), with unity values for $R^{\prime}, Q^{\prime}$, yields

$$
\begin{gathered}
0+0-P^{\prime} \times 1 \times 1 \times P^{\prime}+1=0, \\
P^{\prime 2}=1 .
\end{gathered}
$$

Taking the positive definite value $P^{\prime}=1$ and substituting into (3.2.4.2.6) results in the gainswitching matrix as

$$
G=\left[G_{11} I\right] M_{1}=\left[1^{T} I\right] 1=I .
$$

Coefficients of the controller are computed using the estimates of the plant matrices $\hat{A}_{p}, \hat{B}_{p}$ and those of the model using (3.2.4.2.2-4).

\subsubsection{Experiment Setup and Rotation Monitoring}

As shown in Figure 3.2.6.1, the overall experimental set up contains a Doppler laser vibrometer Polytech PSV-30o to generate the optical waveforms, and a data recovery component (which forms the displacement feedback loop), a real-time control board type dSPACE PPC DS 1103, and the LCR actuator.

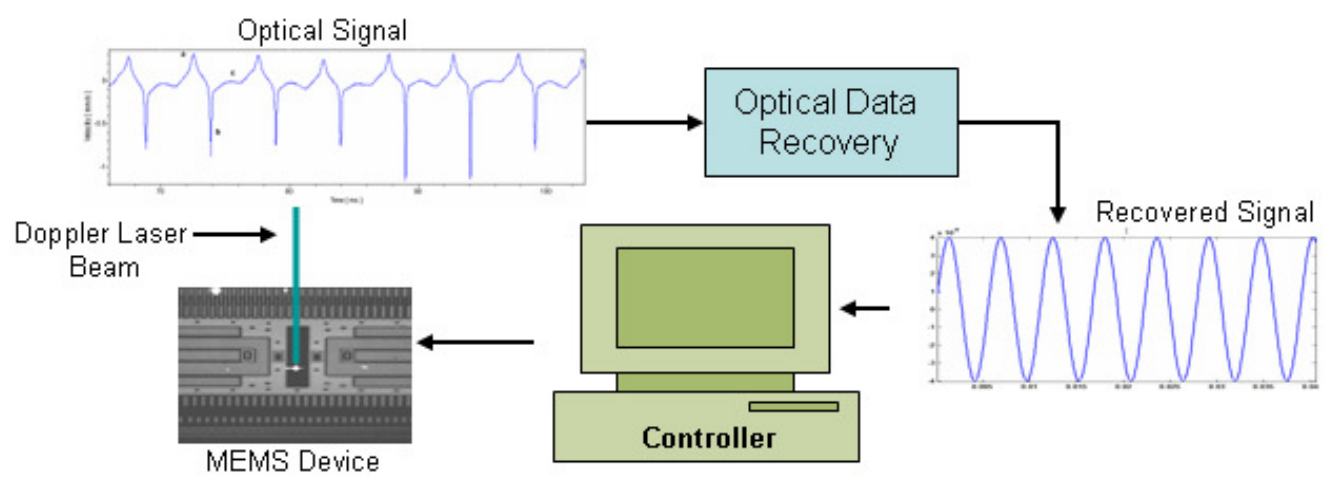

Fig. 3.2.6.1. The experimental setup and signaling flow. The feedback signal is an electric signal. 
As mentioned earlier, the rotation of the shuttle cannot be observed using the optical monitoring technique; however, the x-axis displacement and its effect on the shuttle rotation toward the $y$-axis can be chosen as an indicator of the rotation.

Assuming a linear relationship between the displacement along the x-axis and rotation of the device (the solid shuttle structure), the related pull-in voltage distance along the y-axis is linearly proportional to the displacement along the x-axis with an amplification coefficient $E$. This requires roughly $1 / 3$ of gap " $\mathrm{g}$ " displacement to put the device in a region of instability. Since the adhesion point causes rotation in the LCR's shuttle, combs that are farther from the location of the fault on the shuttle undergo a larger displacement compared to the middle parts and the combs that are closer to the fault location. Figure 3.2.6.2 shows displacement of the shuttle as $d_{x 1}$ and $d_{x 2}$ at the distances $r_{1}$ and $r_{2}$ respectively. The displacement at distance $r_{2}$ from the fault point is higher than what is recorded at the openings of the device. The amplification ratio, which is related to the distance from the adhesion point, is computed as $E \cong \frac{r_{2}}{r_{1}}$. For the manufactured device, this ratio equals $E \cong \frac{700 \mu}{350 \mu}=2$ meaning that the displacement at the comb finger farthest from the fault point is approximately twice of what is recorded by the optical monitoring system at the openings on the shuttle.

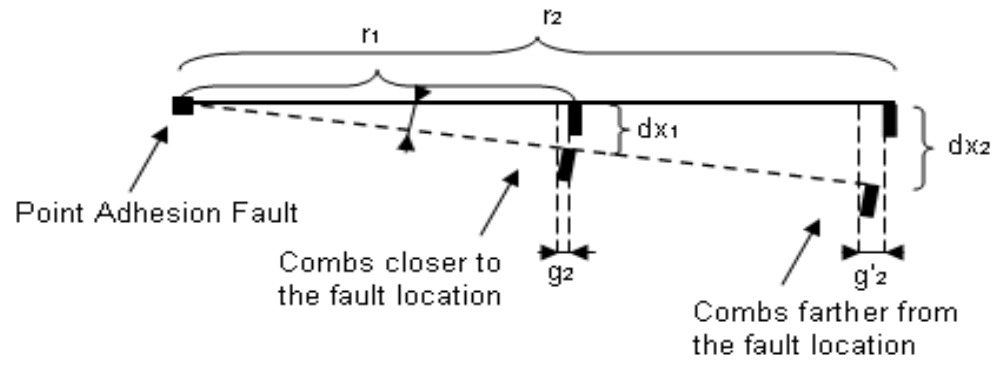

Fig. 3.2.6.2. Shuttle rotation and point adhesion fault location, the displacement on different locations of the shuttle with respect to the fault point is shown. The dimensions were exaggerated for a better demonstration.

The length of the flexure is another important factor for the rotation of the device. For the same rotation angle, longer flexures are displaced farther and consequently are placed closer to the stationary fingers. The gap closing $g_{2}$ is related to both the distance from the fault location and the rotation angle, as $g_{2}=r \theta$. For instance, in a 2-micron gap closing $\left(g_{2}\right)$, the required rotation angle for the comb located at $400 \mu m$ distance from the fault location is $\theta \cong \frac{10.19 \mu}{400 \mu}=0.026^{r}$. A 2micron gap closing accords with a 10-micron displacement along the $\mathrm{x}$-axis and produces a 10.19micron diagonal displacement. This value, as shown in Figure 3.2.6.2, is larger for farther combs, i.e. $g_{2}^{\prime}>g_{2}$. 


\subsubsection{Experiments:}

The successful application of STVSC is demonstrated in two different operational scenarios: a normal operation and a fault condition. In the first experiment, trajectory is controlled for two different cases of short and long displacement under normal conditions and assuming negligible imperfections. In the second experiment the trajectory is controlled beyond pull-in voltage limits for a fabricated LCR device under fault conditions.

Case 1) A device was designed and foundry fabricated with an additional mass on the shuttle to better show the fabrication uncertainties. Thus, the mass in the model was considered at the nominal value whereas the actual device had a $5 \%$ higher mass content. The trajectory tracking performance is shown in Figure 3.2.6.1.1. As the figure shows, the tracking waveform follows the reference closely in healthy condition and with a higher mass quantity on the fabricated LCR. The characteristics of the variable structure controllers and the self-tuning estimators provide for a robust controller in which a LCR device with uncertain parameters due to added mass, aging and operating conditions can be controlled to track the desired reference. The control effort is shown in Figure 3.2.6.1.2 and is compared to the model reference input. The control command is in the same range as that of the reference input, with sign changes to control and track the reference displacement.

In a stable region of operation, the self-tuning component of the controller estimates the system parameter variations during the operation, and then the estimation error is fed back to the controller. The variable structure controller then generates a suitable control command according to the control law (3.2.4.2.1). The adaptation rate of trajectory control for a low frequency reference is shown in Figure 3.2.6.1.3. As the figure shows, the output estimation and the desired reference are completely matched in a short time. The rate of the adaptation is directly related to the adaptation gain of the controller. Over longer displacements beyond the pull-in voltage, assuming negligible imperfections, the shuttle does not experience any rotation and provides a complete displacement under low-frequency excitation as shown in Figure 3.2.6.1.4. The selftuning estimator can also identify system variations. The control effort is shown in Figure 3.2.6.1.5. The control command and the reference input are in almost the same range of variations.

Case 2) In this case (test of the faulty device), an adhesion point fault on the suspension spring of the LCR (see Figures 3.2.2.1 and 3.2.2.2) is designed and fabricated. In addition to its resonant frequency variations, the adhesion point fault limits the displacement of the device. The device therefore operates at lower displacements and lower resonant frequencies. The output voltage of the control hardware also limits the maximum displacement achievable from the device, because, due to greater stiffness of the suspension springs (with an anchor) of the system, higher forces are 
required. The tracking control performance is shown in Figure 3.2.6.1.6. As mentioned earlier, the displacement at the critical comb (the farthest comb) is approximately 2 times longer than the displacement of the center comb. The total gap required for the pull-in limits is one-third of the constant lateral gap, or 0.7 -micron along the y-axis. This value in a linear transformation equals 3.5-micron along the x-axis. Pull-in voltage limit is therefore at 1.67-micron shown with a dashed line in Figure 3.2.6.1.6. This means that any movement beyond this point is in an unstable region of operation, in which the electrostatic force becomes much larger than that of the restoring energy of the suspension spring. Figure 3.2.6.1.6 also demonstrates the capabilities of STVSR in controlling the small gap closing in LCRs. The control command in this case is shown in Figure 3.2.6.1.7. The controller changes the sign of the applied voltage to have the device pulled back to the region of stability and follow the reference trajectories.

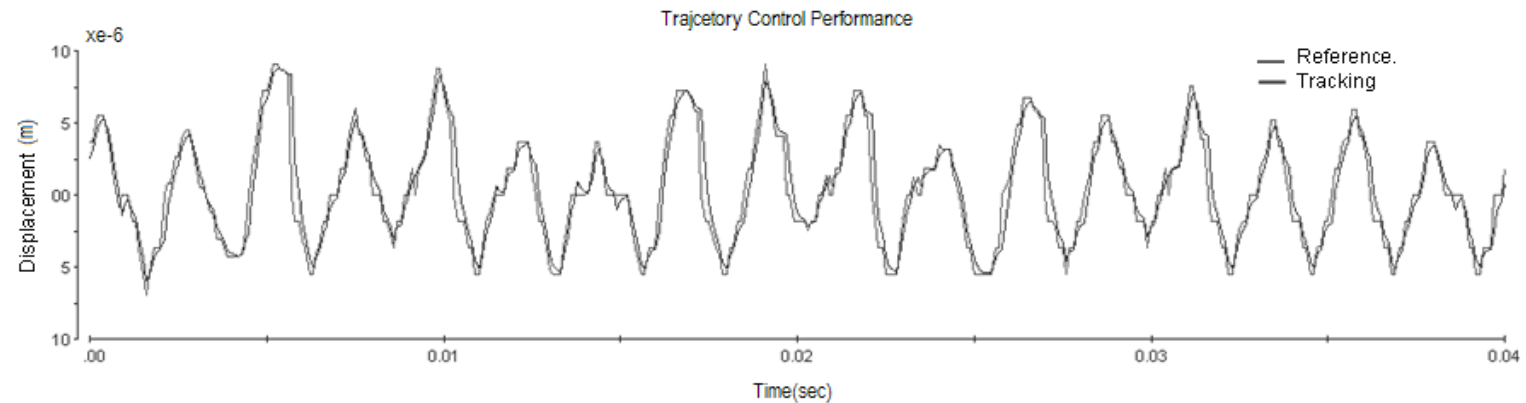

Fig. 3.2.6.1.1. Tracking control and reference waveforms in terms of micrometer.

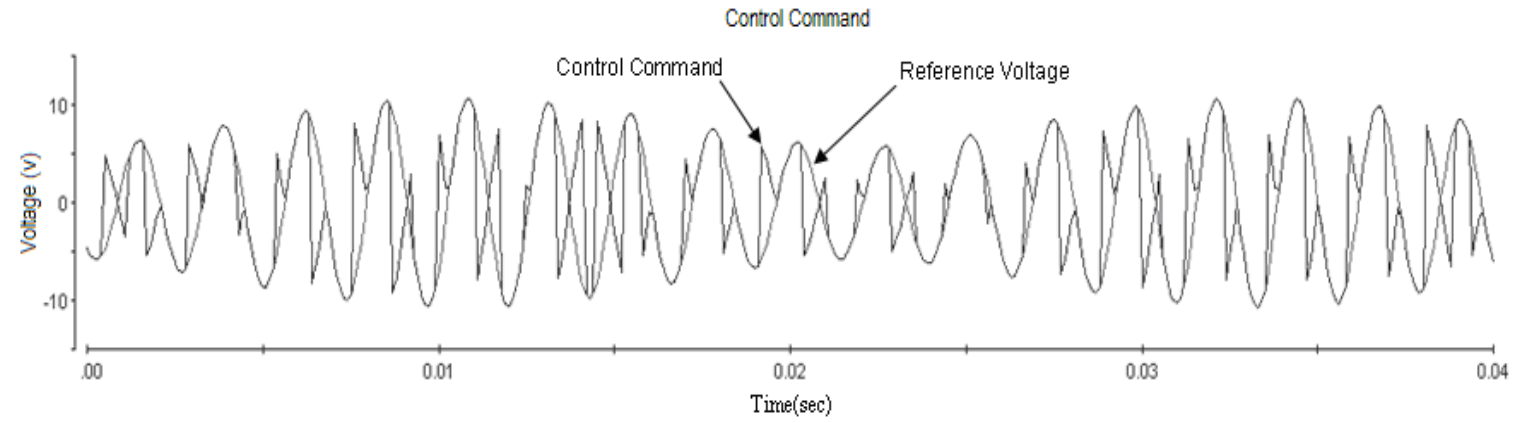

Fig. 3.2.6.1.2. Control command and the reference voltage.

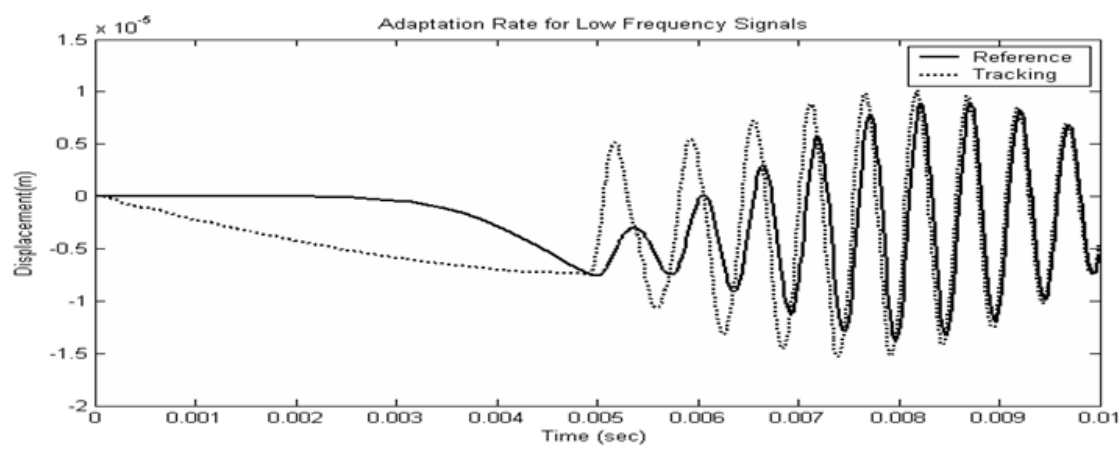

Fig. 3.2.6.1.3. Adaptation of the STVSC for a low frequency waveform. 


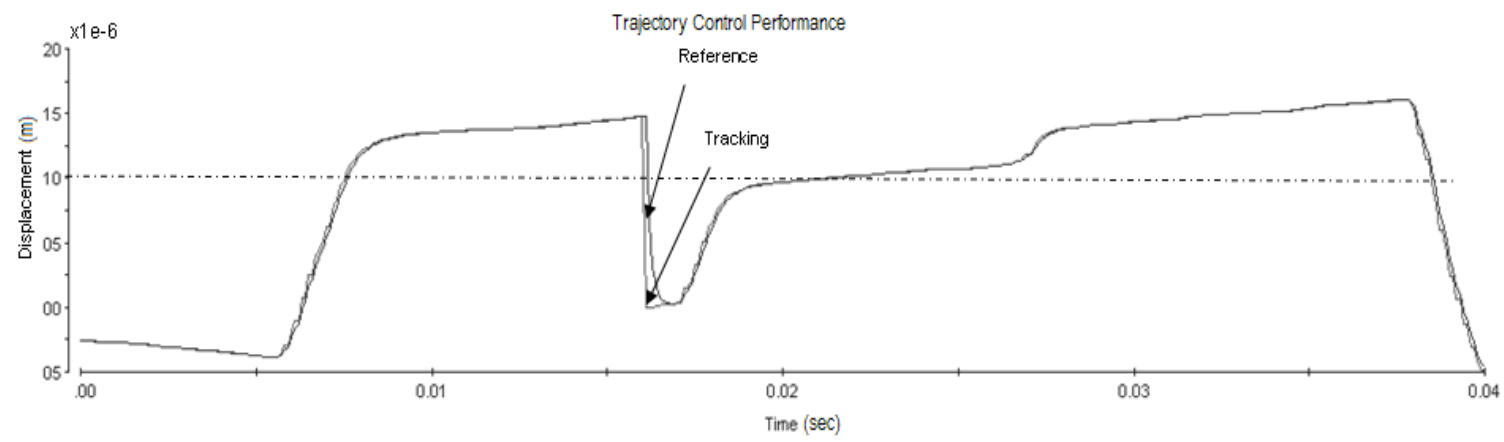

Fig. 3.2.6.1.4. Displacement and tracking control beyond the pull-in voltage point in unstable region of operation. The transient tracking control in this region is shown in dashed circles.

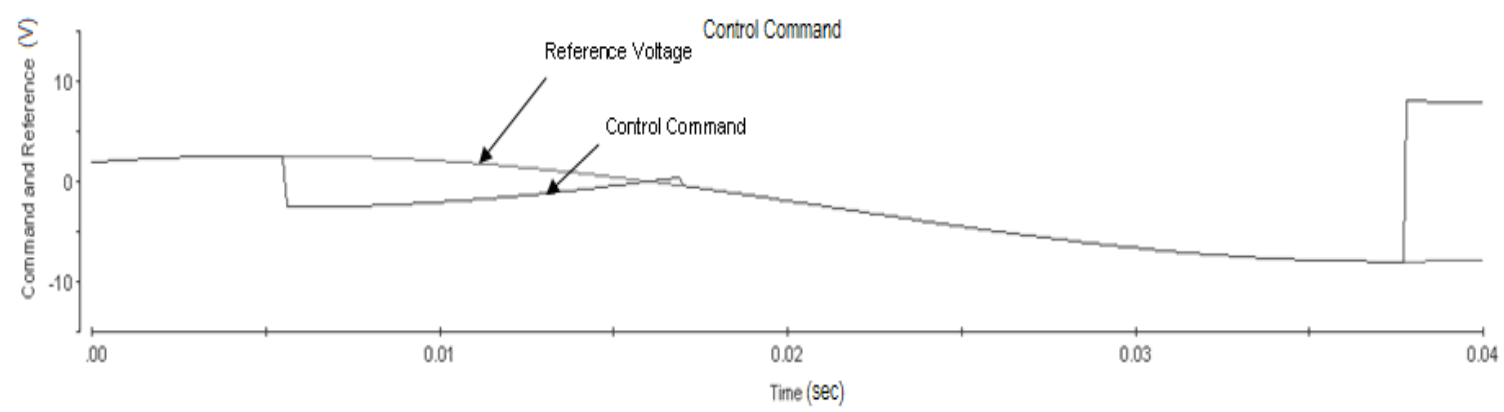

Fig. 3.2.6.1.5. Reference and control command of long displacement transient tracking control.

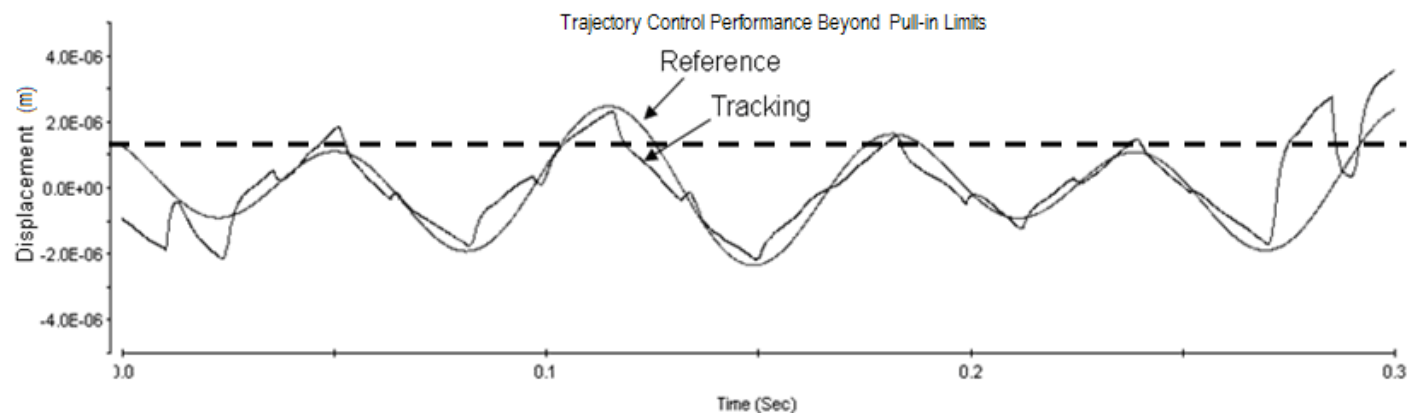

Fig. 3.2.6.1.6. Trajectory control of the faulty device. Fault was as a point adhesion on the suspension spring of Lateral Comb Resonator. Displacement is 2.1 times larger for the farthest comb from the fault location. Dashed line shows the Pull-in voltage limit.

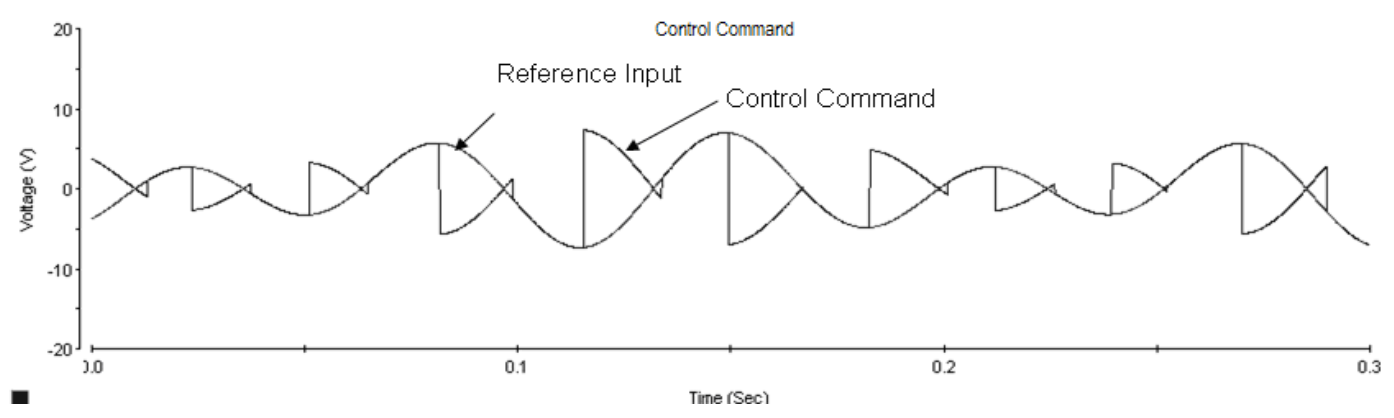

Fig. 3.2.6.1.7. Control effort and the reference input in case of the point adhesion fault on the device. 


\subsection{Online Parameter Identification and Control of MEMS}

\subsubsection{Online parameter identification:}

In the plants with unknown parameters or with time varying parameters, the identification of the parameters helps to obtain a better control performance. In parameter estimators, the parameters of the system and their variations are continuously monitored and their instantaneous values are used for controller gains resulting in self-tuning controllers. There are several online parameter identification techniques such as gradient, Least Square, Recursive Least Square, and Recursive Least Square with different forgetting factor configurations. Each of these techniques are chosen regarding to the system dynamics and parameter characteristics. In case of time-varying parameters and in the presence of noise, the FFRLS can be used (See appendix B). This technique is described in the following and is applied to a MEMS LCR device to identify the parameters of the device. Then the Parameter Identifier (PI) is applied to a Model Reference Adaptive control to form the indirect adaptive control technique.

\subsubsection{Recursive Least Square with Forgetting Factor:}

Forgetting factor determines the weight of the recent input-output values with respect to the old data in the system identification. In this method, the weight can compensate for the rate of the variation and noise in the system. For system identification, the system should be represented in the parametric form, and then forgetting factor can be applied to find out the parameters of the system. The control approach requires gain-calculating functions from the estimated parameters which can be found from equalizing the resultant transfer functions. The steps are illustrated as follows:

Step 1: parametric model. Since the MEMS was modeled as a mass-spring-damper system in (1.3.8) as

$$
F_{e}=m \ddot{x}+\beta \dot{x}+2 k_{s} x+F_{d} .
$$

Replacing the electrostatic force from (1.3.2), results in

$$
\left(s^{2}+\frac{\beta}{m} s+\frac{2 k_{s}}{m}\right) y=\frac{K}{m} V_{s},
$$


where $V_{s}$ is the input and $y$ is the output. Considering $a p_{1}=\frac{\beta}{m}, a p_{0}=\frac{2 k_{s}}{m}$ and $K_{p}=\frac{K}{m}$ results in the following transfer function

$$
\frac{y}{V_{s}}=\frac{K_{p}}{s^{2}+a p_{1} s+a p_{0}} .
$$

The system (3.3.2.2) can be written as

$$
s^{2} y=\frac{K}{m} V_{s}-\frac{\beta}{m} s y-\frac{2 k_{s}}{m} y .
$$

Filtering both sides by a Monic polynomial $\Delta(s)=(s+\lambda)^{n}$ of the same order as the system (in this case 2) results in

$$
\frac{s^{2}}{\Delta(s)} y=\left[\frac{K}{m}, \frac{\beta}{m}, \frac{2 k_{s}}{m}\right] \cdot\left[\frac{1}{\Delta(s)} V_{s},-\frac{s}{\Delta(s)} y,-\frac{1}{\Delta(s)} y\right]^{T} .
$$

Consider the following values

$$
\begin{gathered}
z=\frac{s^{2}}{\Delta(s)} y \\
\theta=\left[\frac{K}{m}, \frac{\beta}{m}, \frac{2 k_{s}}{m}\right]^{T}=\left[K_{m}, a p_{1}, a p_{2}\right]^{T} . \\
\phi=\left[\frac{1}{\Delta(s)} V_{s},-\frac{s}{\Delta(s)} y,-\frac{1}{\Delta(s)} y\right]^{T}
\end{gathered}
$$

Then the formulation can be considered as

$$
z=\theta^{T} \phi
$$

where $\theta$ is the vector of unknowns and is being identified by a proper technique.

\section{Step 2: FFRLS}

The parameters are updated as the following technique

$$
\dot{\theta}=P e \phi, \quad \theta(0)=\theta_{0},
$$


where $\theta_{0}$ is the initial values of the parameters, and $e$ is the estimation error computed as

$$
e=\frac{z-\theta^{T} \phi}{m_{s}^{2}}
$$

The covariance $P$ is updated as following

$$
\dot{P}=\beta P-P \frac{\phi \phi^{T}}{m_{s}^{2}} P, \quad P(0)=P_{0},
$$

where $\beta>0$ is the forgetting factor, and $m_{s}^{2}$ is the normalizing signal, defined as

$$
m_{s}^{2}=1+\alpha \phi^{T} \phi
$$

with a positive $\alpha>0$ coefficient.

\section{Step 3: controller design:}

In this section, the estimated parameters of the system are applied to design the controller. The controller is designed according to Figure 3.3.2.1 for a second order system:

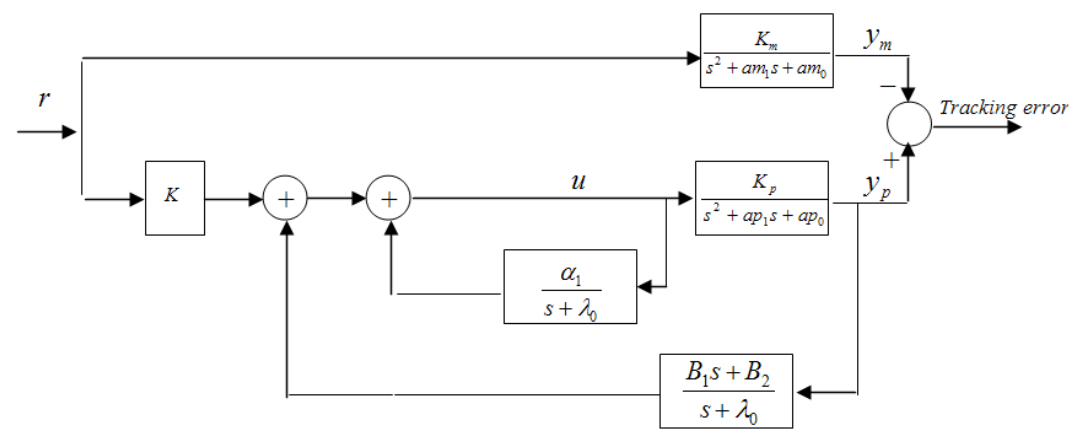

Figure 3.3.2.1, Schematic block diagram of self-tuning adaptive controller

The control law can be written as

$$
u=\frac{\alpha_{1}}{s+\lambda_{0}} u+\frac{B_{1} s+B_{2}}{s+\lambda_{0}} y_{p}+K r
$$

Or

$$
u=\frac{B_{1} s+B_{2}}{s+\lambda_{0}-\alpha_{1}} y_{p}+K \frac{s+\lambda_{0}}{s+\lambda_{0}-\alpha_{1}} r .
$$


In order to have the same output generated from the model and the plant, the transfer function of the model from $y_{m}$ to $r$ should be equal to the controller and the plant combined from $y_{p}$ to $r$. applying the controller to the plant the output is written as

$$
\frac{y_{p}}{r}=\frac{K_{p} K\left(s+\lambda_{0}\right)}{\left(s+\lambda_{0}-\alpha_{1}\right)\left(s^{2}+a p_{1} s+a p_{0}\right)-K_{p}\left(B_{1} s+B_{2}\right)} .
$$

Step 4: Equalizing with the model's transfer function yields

$$
\frac{K_{p} K\left(s+\lambda_{0}\right)}{\left(s+\lambda_{0}-\alpha_{1}\right)\left(s^{2}+a p_{1} s+a p_{0}\right)-K_{p}\left(B_{1} s+B_{2}\right)}=\frac{K_{m}}{s^{2}+a m_{1} s+a m_{0}} .
$$

The controller coefficients are computed as

$$
\begin{gathered}
K=\frac{K_{p}}{K_{m}}, \\
\alpha_{1}=a p_{1}-a m_{1}, \\
B_{1}=\frac{1}{K_{p}}\left(a p_{2}+\left(\lambda_{0}-\alpha_{1}\right) a p_{1}-\lambda_{0} a m_{1}-a m_{2}\right), \\
B_{0}=\frac{1}{K_{p}}\left(a p_{2}\left(\lambda_{0}-\alpha_{1}\right)-\lambda_{0} a m_{2}\right) .
\end{gathered}
$$

\subsubsection{Parameter Identification Simulations:}

Considering the forgetting factor as $B=10$, and the normalizing coefficient at equation (3.3.2.11)at $\alpha=10$, the parameters of the plant transfer function are identified in a short time as shown in Figures 3.3.3.1-3. 


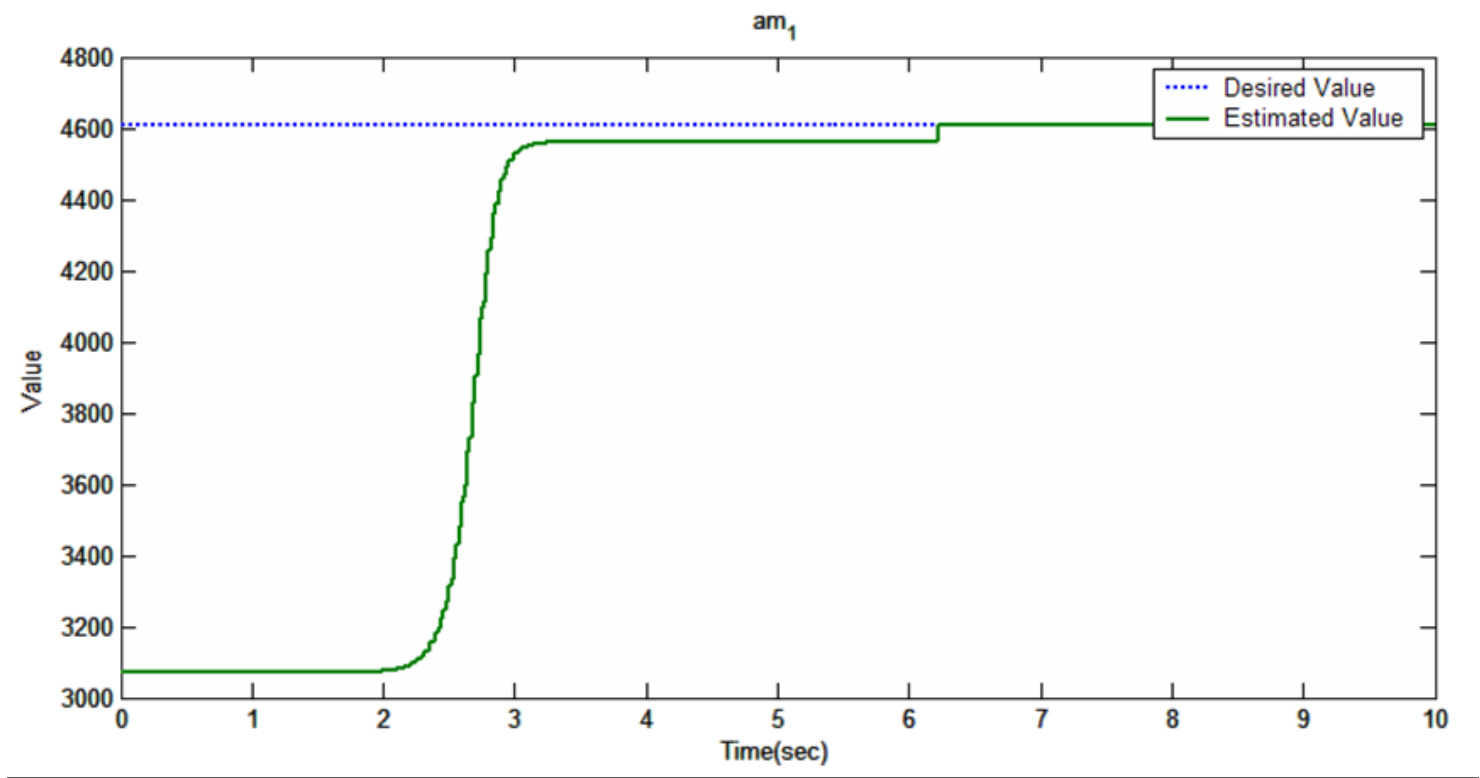

Figure 3.3.3.1, Model parameter identification at $B=10$ and $\alpha=10$

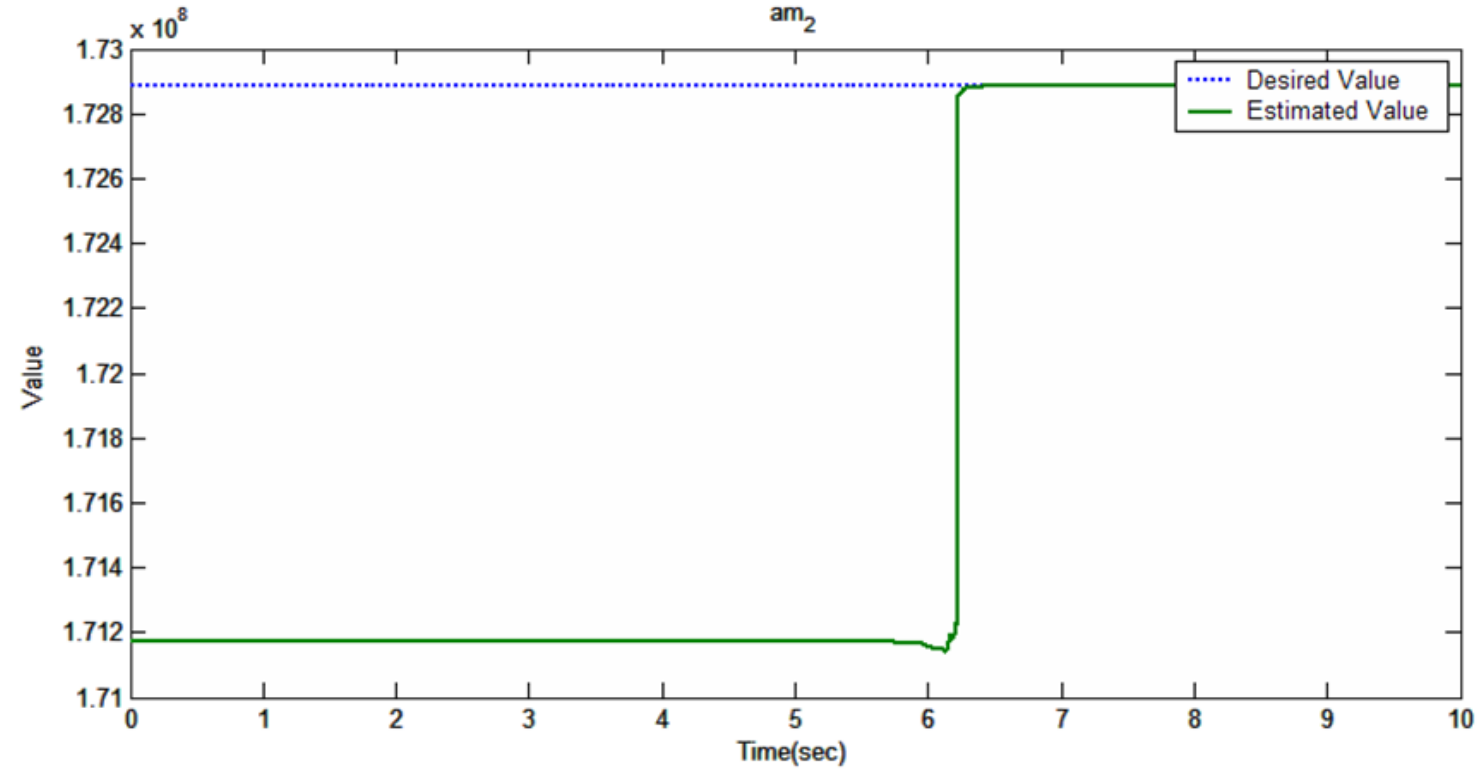

Figure 3.3.3.2, Parameter identification at $B=10$ and $\alpha=10$ 


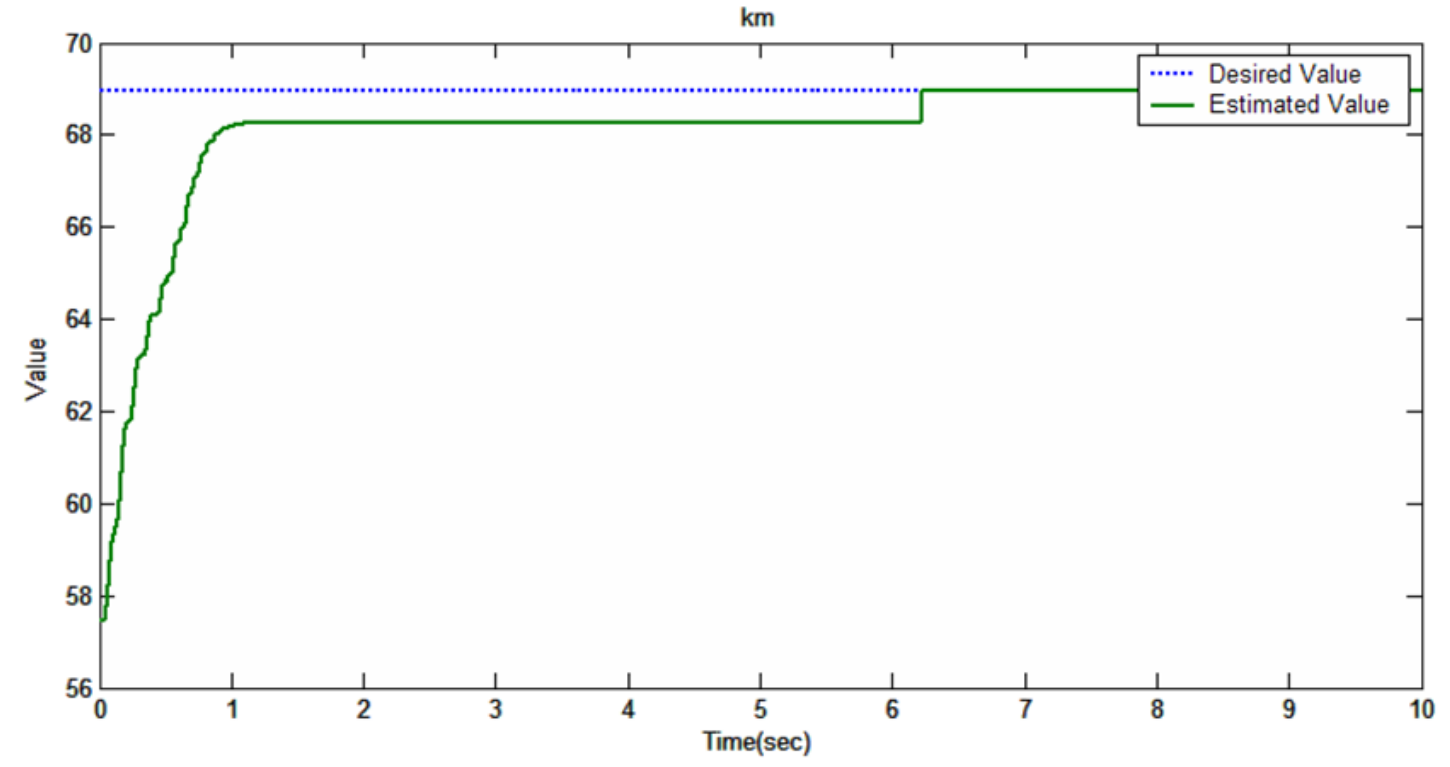

Figure 3.3.3.3, high frequency gain identification at $B=10$ and $\alpha=10$

The performance of the Parameter Identification in combination with MRAC controller is shown in Figure 3.3.3.4. As the figure shows, the plant's output follows the desired trajectory very closely in a short time. Figure 3.3.3.5 shows the control effort comparing to the reference input to the system. As the figure shows, the control effort decreases to compensate for the uncertainties in the system.

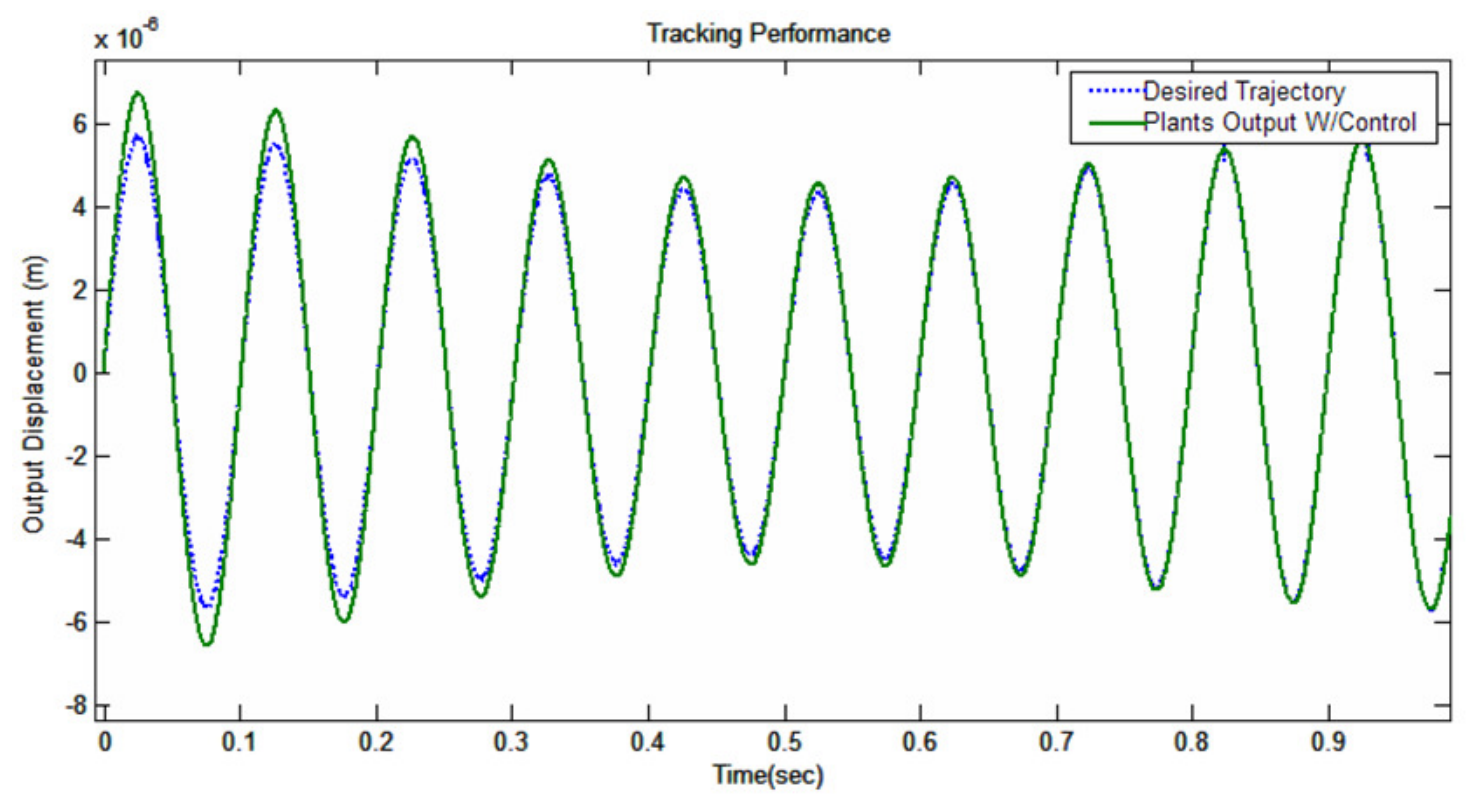

Figure 3.3.3.4, Tracking performance of the controller at $B=10$ and $\alpha=10$ 


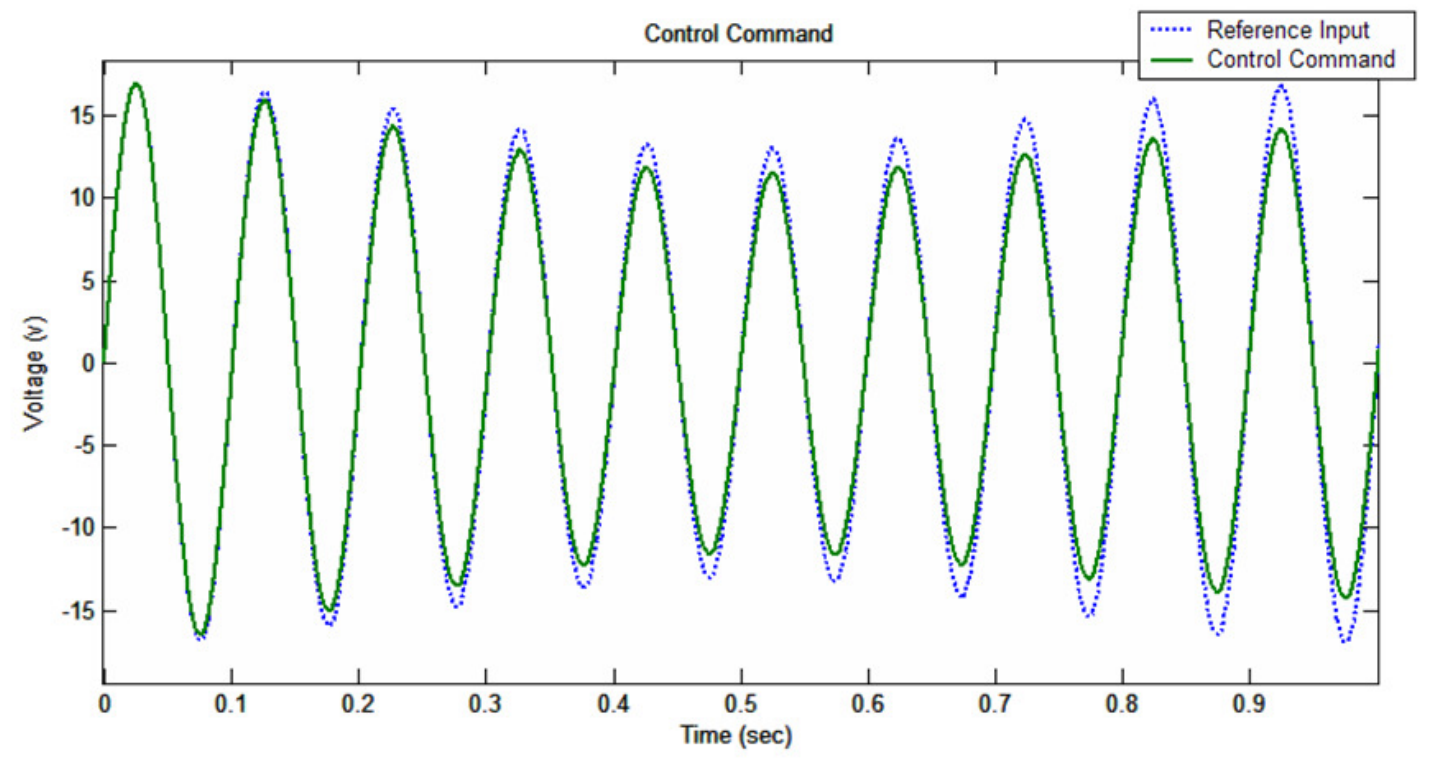

Figure 3.3.3.5, model reference input and control command at $B=10$ and $\alpha=10$

As the Parameter Identification coefficients increased to higher numbers, the convergence rate was also increased and the parameters were identified very quickly at $B=30$ and $\alpha=10$. Figures 3.3.3.6-8 show the performance of the parameter identification. As they show, higher rate of convergence comparing to the Figures 3.3.3.1-3 at higher forgetting factor. Note that the alpha value insures the bounded input output signals.

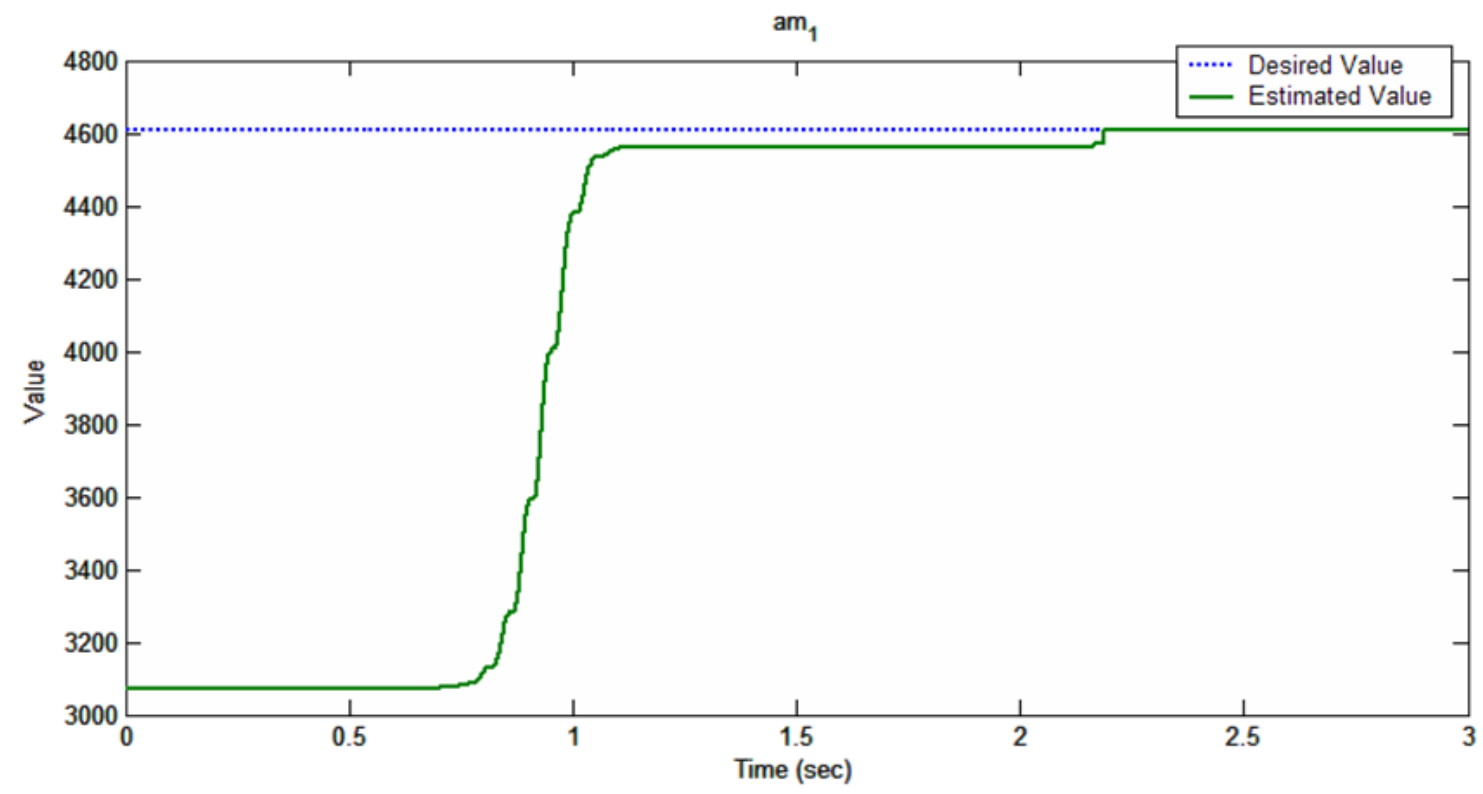

Figure 3.3.3.6 Parameter identification at $B=30$ and $\alpha=10$ 


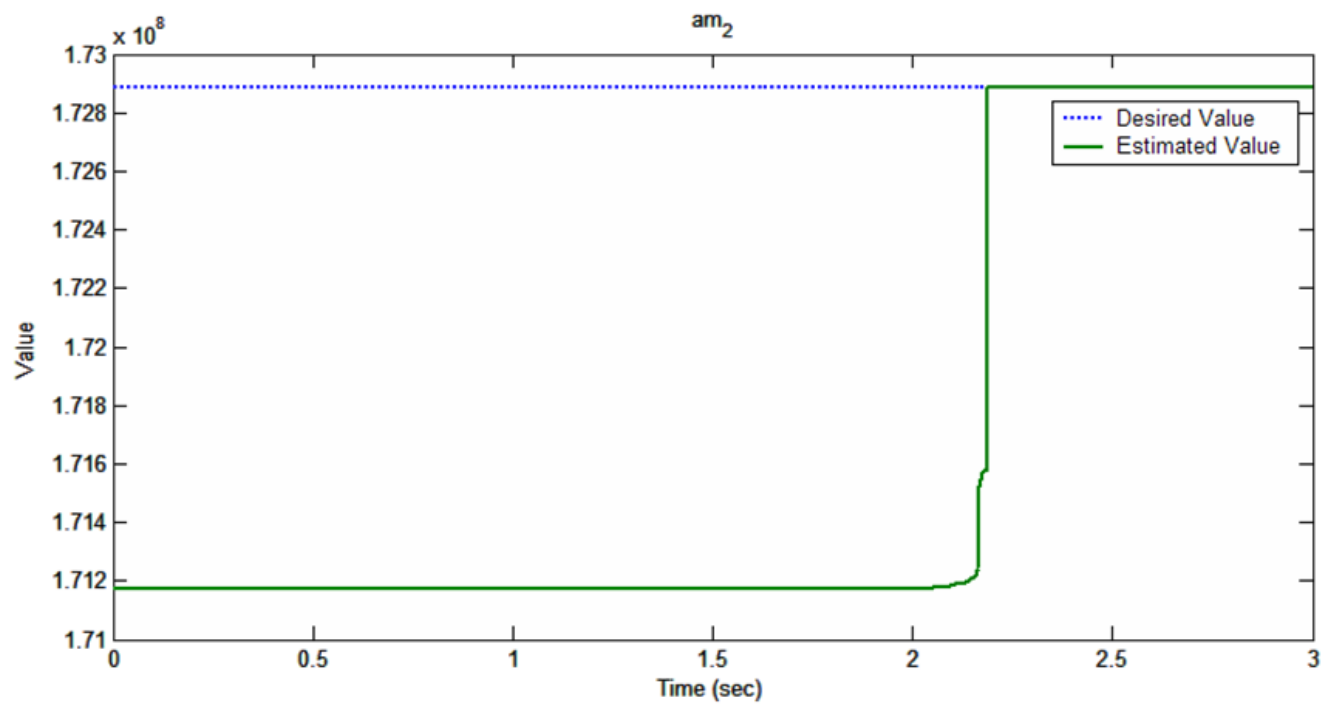

Figure 3.3.3.7, Parameter identification at $B=30$ and $\alpha=10$

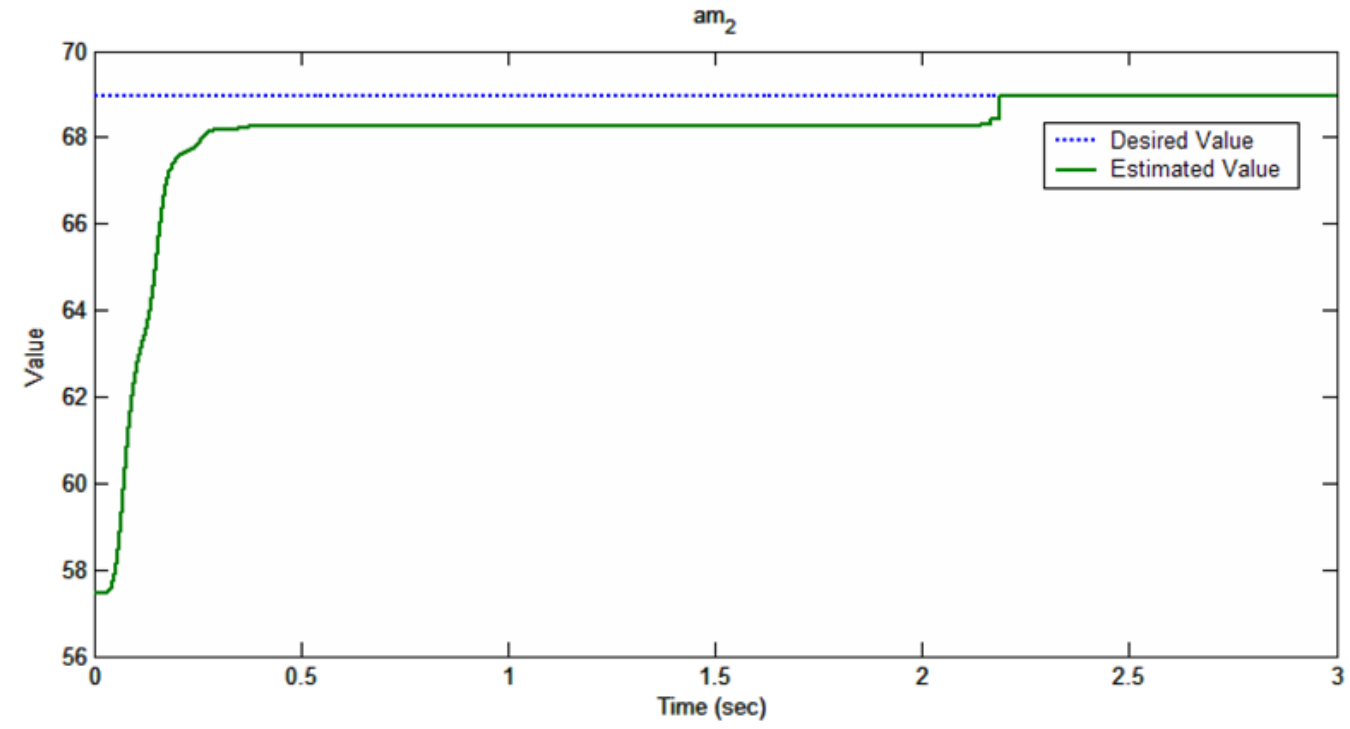

Figure 3.3.3.8, high frequency gain identification at $B=30$ and $\alpha=10$

The tracking control performance increases at higher values as it is shown in Figure 3.3.3.9. The control command is shown in Figure 3.3.3.10 in this case. 


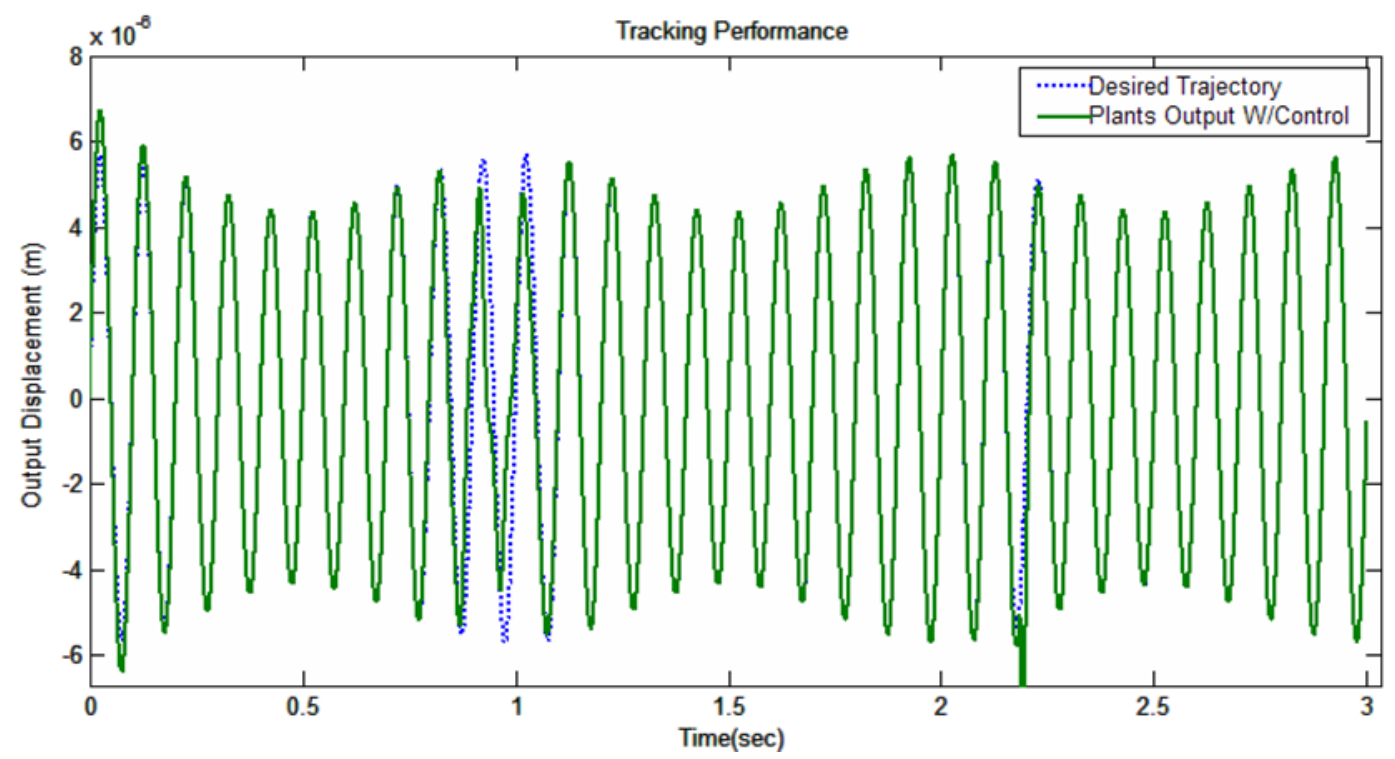

Figure 3.3.3.9, Tracking performance of the controller at $B=30$ and $\alpha=10$

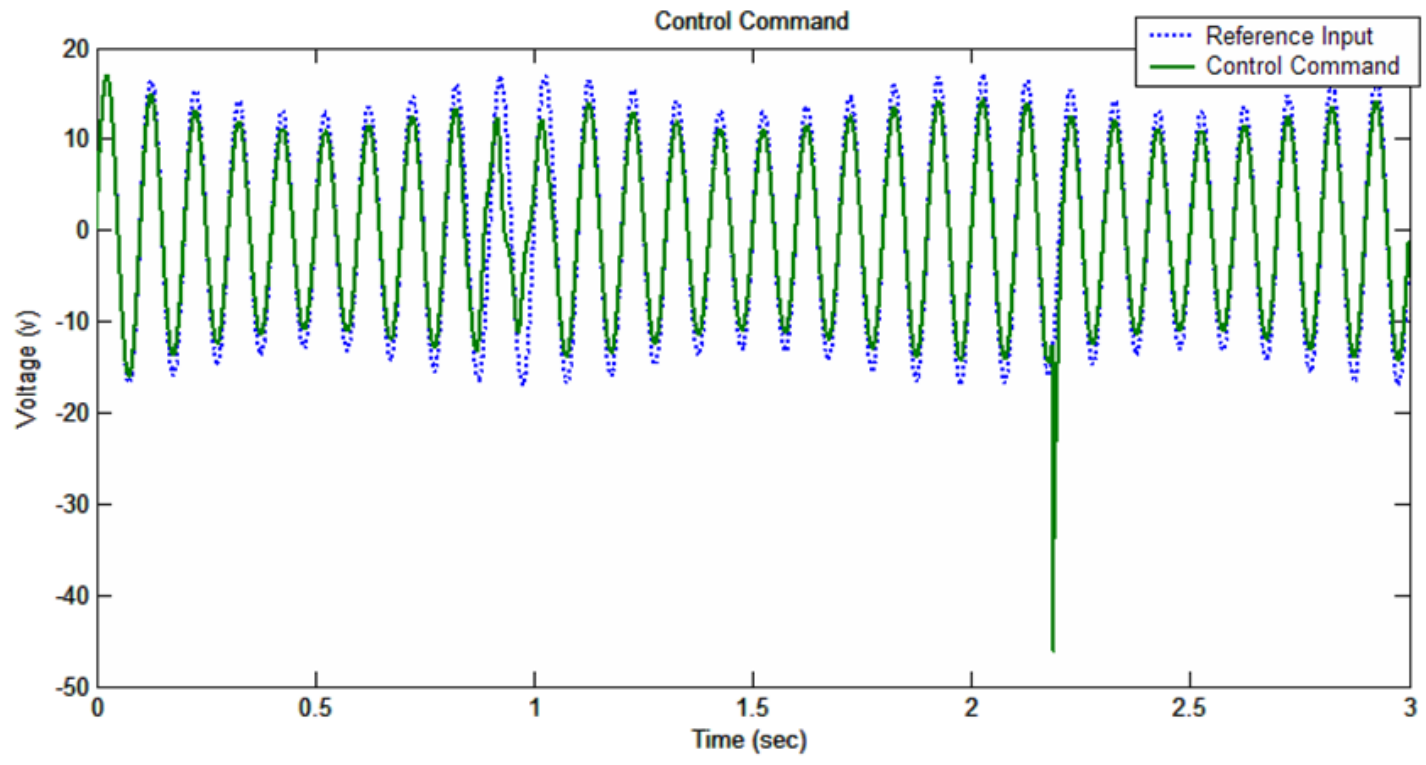

Figure 3.3.3.10, model reference input and control command at $B=30$ and $\alpha=10$ 


\subsection{Concluding Remarks}

This chapter has demonstrated a successful implementation of control strategies to faulty MEMS devices to enhance their reliability of operation and solve for the structure rotation and pull-in voltage control. Simulations and experiments were conducted to enhance reliable operation of Lateral Comb Resonators by utilizing Model Reference Adaptive Controllers. The most probable types of fault that may occur in microsystems were fabricated at the chip level of LCR in the form of mass variation and spring constant changes. In the case of mass change, the effects of aging and environmental conditions were also considered. Mass variation by itself required low control efforts, whereas the case of also considering the effects of aging and environmental conditions which required almost $80 \%$ higher efforts than input-reference. Suspension spring defects required 20\% and 46\% higher efforts for broken trusses and adhesion point fault respectively. The controller provided a reliable fault-tolerant trajectory control by improving the utilization of the device in harsh and fault conditions.

Tracking control of MEMS Lateral Comb Resonators was also studied under parameter uncertainties and fault, where the pull-in voltage effect was considered as a limitation. Pull-in voltage occurred in the gap closing (between the stationary and shuttle fingers) orthogonal to the main direction of displacement in the case of shuttle rotation. Self-Tuning Variable Structure Controllers (STVSC) were applied to control the device under healthy and fault conditions. Manufacturing imperfections were also considered in the form of mass variation $-\mathrm{a}+5 \%$ additional mass, which was fabricated on the shuttle of an LCR and used for trajectory control, applying the same controller structure. Experimental results demonstrated the effectiveness of the STVSC for trajectory control applications under parameter uncertainties in a healthy device. The experiments also verified the capabilities of the controller in the case of a low-frequency longdisplacement operation and beyond pull-in voltage limits in the presence of the fault in the microsystem. 


\section{Chapter 4,}

\section{A New Input-Output Controller Design and Application in Control of MEMS Comb Resonators}

Conventional adaptive controllers, such as Model Reference, make use of several input-output filters for pole placement and control of non-minimum phase systems. Combined adaptive controllers and variable structure systems require a varying gain-switching matrix to remain on the sliding surface. In these controllers, state space variables of plant are used in the control command, and exact values of the system parameters are required for the controller gain and switching-matrix computation. This chapter introduces a new type of adaptive controller without the need of input-output signal filters and gain-switching matrix computation. The control law depends only on the input reference and output of the plant and model. The main advantages of the new controller are: a) its simple control law and adaptation technique without the need of filters, b) the controller does not depend on the system state space variables except its I/O sets, and c) fast adaptation rate is obtained at low gains. The structure of the controller and its adaptation technique for Strictly Positive Real (SPR)/Non-SPR systems are illustrated.

\subsection{Introduction}

Adaptive controllers were earlier introduced to control the systems with varying parameters such as autopilots (Hung, Gao \& Hung. 1993), (Osburn, Whitaker, Kezer.-), (Parks, 1966). In the same family of controllers, Model Reference Adaptive Control (MRAC) systems with robust tracking capabilities were designed for SPR systems and systems with higher relative degrees. History of adaptive controller's evolution and their applications are illustrated in (Astrom, Wittenmark. 1995), (Hung, Parks. 1973), (Gambier. 2004), (Parks. 1964), (Dressler. 1967), (Price. 1970), (Monopoli., Gilbert, Thayer. 1968) and appendix B of this dissertation. The main drawback of the conventional adaptive controllers is the numerous filters required for input-output sets of high order systems for zero-pole placements and parameter identification. They also require Persistently Exciting (PE) reference signals to guarantee their asymptotic stability. They have also shown some problems with systems of bad dynamics (Itkis. 1976). Switching and variable structure systems offered a solution for the need of PE signals (Liu, Skormin. 1988), (Hsu, Araujo. 1994) (Bertran, Lopez. 1995) (Mitic. 1997) (Masuda, Inque. 1995) (Hsu, Lizarralde, Arajuo. 1997). Variable Structure Systems (VSS), first introduced in early 1950s in Soviet Union (Hung, Gao, 
Hung. 1993), were later benefited from the adaptive controllers and provided robust control systems , (Hung, Parks. 1973), (Itkis. 1976), (Hsu. 1988). A combination of adaptive systems with VSS have found applications in robot manipulators (Itkis. 1976), (Hsu. 1988), (Tsu, Xu and Shum. 1991), but complexity of their algorithms mainly the gain-switching matrix computations, the need for exact parameter values of the system for gain computations, and dependency of their control law on the system states (see section 3.2.4.2) caused the loss of their popularity comparing to conventional controllers.

This chapter presents a promising adaptive control approach and gain adaptation law to eliminate the need for the input-output filter designs. The new controller is an input-output type of system with Lyapunov stable gain adaptation laws. The controller is designed for Strictly Positive Real systems (SPR) and the systems with higher relative degrees utilizing the error augmentation technique. The performance of the controller is demonstrated in trajectory control of MEMS Lateral Comb Resonators.

\subsection{New Controller Design}

\subsubsection{Case 1, Relative Degree One (SPR)}

In an uncertain parameter, controllable, of an arbitrary order with relative-degree one, and Single-Input Single-Output system (plant), with strictly proper transfer function, where only the sets of input-output are measurable and bounded, the control law satisfying Filippov condition $\dot{s}=0$ is defined as

$$
C=k_{p} R_{p}+k_{e}\left(R_{m}-R_{p}\right)+k_{r} r
$$

where $R_{m}, R_{p}$ are the model's and plant's response (output) to the reference input $r$ and control command $C$ respectively, and $k_{p}, k_{e}, k_{r}$ are the controller gains that are adjusted simultaneously according to a gain adaptation law.

In a perfect matching control, the coefficients $k_{P}, k_{e}, k_{r}$ exist such that the plant's output is matched to that of the model, result in zero tracking error. The existing conditions of the control law and sliding surface are discussed in (Osburn, Whitaker, Kezer.-), (Parks, 1966). In the new controller, the switching matrix should be calculated such that zero error conditions hold i.e.

$$
\lim _{t \rightarrow \infty} e(t)=0
$$

Considering the estimated values for the control coefficients, the equivalent control command 
$\hat{C}$ can be defined as

$$
\hat{C}=\hat{k}_{P} R_{P}+\hat{k}_{e} e+\hat{k}_{r} r
$$

where coefficients of $\hat{k}_{P}, \hat{k}_{e}, \hat{k}_{r}$ are estimated values and are computed according to the following theorem.

THEOREM 1: In a SISO and SPR system, the control law of (4.2.1.3) according to the following control gain adaptation techniques (4.2.1.4-6) is asymptotically stable.

$$
\begin{aligned}
& \dot{\hat{k}}_{r}=-P_{0} \operatorname{sgn}(s) r \\
& \dot{\hat{k}}_{P}=-P_{0} \operatorname{sgn}(s) R_{p} \\
& \dot{\hat{k}}_{e}=-P_{0} \operatorname{sgn}(s) e
\end{aligned}
$$

where the sliding surface is defined as $s=G e=0$ and $G$ is the gain-switching matrix, and sliding mode surfaces are the set of $s^{T}=\left(s_{1}, \ldots, s_{n}\right)$.

Proof: Consider the following Lyapunov function with positive gain $P_{0}>0$ as

$$
V=\frac{1}{2} e^{2}+\frac{|s|}{2 P_{0}}\left(\tilde{k}_{e}^{2}+\tilde{k}_{r}^{2}+\tilde{k}_{P}^{2}\right)
$$

satisfying

$$
\begin{aligned}
& \text { i) } V>0, \\
& \text { ii) } V(\overline{0}, t)=0,
\end{aligned}
$$

where $(\sim)$ sign shows the estimation error of each component. Consider the system in sliding mode and note that the sliding surface exists even at zero-tracking error condition (Osburn, Whitaker, Kezer.-), (Parks, 1966); Now, at different sliding directions, the time derivative of the Lyapunov function can be computed in two cases as

a) If $\operatorname{sgn}(s)>0$, then 


$$
\begin{aligned}
& \dot{V}=e \dot{e}+\frac{s}{2 P_{0}}\left(2 \dot{\hat{k}} \hat{k}_{e}-2 \dot{\hat{k}}_{e} k_{e}+2 \dot{\hat{k}}_{r} \hat{k}_{r}-2 \dot{\hat{k}}_{r} k_{r}+\right. \\
& \left.2 \dot{\hat{k}}_{p} \hat{k}_{p}-2 \dot{\hat{k}}_{p} k_{p}\right)+\frac{\dot{s}}{2 P_{0}}\left(\tilde{k}_{e}^{2}+\tilde{k}_{r}^{2}+\tilde{k}_{P}^{2}\right)
\end{aligned}
$$

Replacing with the gain adaptation yields

$$
\dot{V}=e\left(\tilde{k}_{e} e+\tilde{k}_{r} r+\tilde{k}_{p} R_{p}\right)-\frac{s}{2 P_{0}}\left(\tilde{k}_{e} e+\tilde{k}_{r} r+\tilde{k}_{p} R_{p}\right)+0
$$

b) If $\operatorname{sgn}(s)<0$ then

$$
\begin{aligned}
& \dot{V}=e \dot{e}-\frac{-s}{2 P_{0}}\left(2 \dot{\hat{k}}_{e} \hat{k}_{e}-2 \dot{\hat{k}}_{e} k_{e}+2 \dot{\hat{k}}_{r} \hat{k}_{r}-2 \dot{\hat{k}}_{r} k_{r}+\right. \\
& \left.2 \dot{\hat{k}}_{p} \hat{k}_{p}-2 \dot{\hat{k}}_{p} k_{p}\right)-\frac{\dot{s}}{P_{0}}\left(\tilde{k}_{e}^{2}+\tilde{k}_{r}^{2}+\tilde{k}_{P}^{2}\right)
\end{aligned}
$$

Replacing with the gain adaptation yields

$$
\dot{V}=e\left(\tilde{k}_{e} e+\tilde{k}_{r} r+\tilde{k}_{p} R_{p}\right)-\frac{s}{2 P_{0}}\left(\tilde{k}_{e} e+\tilde{k}_{r} r+\tilde{k}_{p} R_{p}\right)+0
$$

Considering the, (4.2.1.9) and (4.2.1.11), the following expression is implied

$$
\dot{V}=\left[\tilde{k}_{e} e^{2}-\tilde{k}_{e} s e\right]+\left[\tilde{k}_{r} e r-\tilde{k}_{r} s r\right]+\left[\tilde{k}_{p} e R_{p}-\tilde{k}_{p} s R_{p}\right]
$$

Replacing the sliding surface condition results in the following equation

$$
\dot{V}=[I-G] e\left[\tilde{k}_{e} e+\tilde{k}_{r} r+\tilde{k}_{p} R_{p}\right]
$$

Considering a constant identity value for gain-switching matrix (i.e. $G=I$ ) in (4.2.1.13), results in $\dot{V}=0$, which satisfies the La Salle's global invariant set theorem [(Slotine, pp.73)]; therefore, the control law (4.2.1.3) with gain adaptation of (4.2.1.4-6) result in an asymptotically stable system.

As it is seen, the proposed control law is a function of input-output signals and gains, which do not depend on the state variables and system parameter values. The controller is Lyapunov stable and makes no use of any filter in the input and output signals. 


\subsubsection{Case 2, Relative Degree Greater Than One}

The systems with relative degree greater than one do not satisfy the self-stability properties of an SPR system. Therefore, a different approach is required to control these systems. Augmented error techniques are usually used to ensure the stability of non-SPR systems. In the new design, the augmented error signal is used as (Slotine, Li. 1991).

$$
\eta(t)=\theta^{T}\left[G_{m} \omega\right]-\left[G_{m} \theta^{T}\right] \omega,
$$

where $G_{m} \equiv G_{m}(s)$ is the transfer function of the model, $\theta$ is matrix of the controller coefficients, and $\omega$ is matrix of signals defined as

$$
\begin{gathered}
\theta=\left[\begin{array}{lll}
k_{p} & k_{e} & k_{m}
\end{array}\right]^{T}, \\
\omega=\left[\begin{array}{c}
r \\
e \\
R_{p}
\end{array}\right] .
\end{gathered}
$$

Lemma, (Slotine, Li. 1991), the augmented error is defined as the summation of tracking error and a weighted augmentation signal as

$$
\varepsilon(t)=e(t)+e_{a}(t) \eta(t)
$$

where $e_{a}(t)$ is the weight of the augmented signal and is updated as (Slotine, Li. 1991)

$$
\dot{e}_{a}=-\frac{\Gamma \varepsilon \eta}{1+\omega^{T} \omega}
$$

where $\Gamma>0$ is a positive number. (The augmentation signal and weight values are time dependent functions). The proof of stability and derivation of (4.2.2.5) can be found in (Slotine, Li. 1991), (Monopoli 1974).

As mentioned earlier, in sections II and III, other types of controllers require suitable filter design for input and output signals, but the proposed controller does not require any of the filter designs. VSMRC requires gain switching matrix computations in each step, but the new controller operates with a fixed gain-switching matrix and makes use of the augmentation error technique for non-SPR systems. 


\subsection{Simulation and Experiment Results}

\subsubsection{Performance Analysis}

In this section, the application and performance of the new design controller are investigated and compared with the controllers of the same class such as Models Reference Adaptive Controller and Variable Structure Model Follower Controllers as Regulators, Tracking, Double Integrator's Control, and applications in MEMS.

Regulator, The application of the controller as a regulator is demonstrated in Figure 4.3.1.1. A plant with an unstable pole at $s=0.2$ is controlled to generate a constant output as $y_{M}=2$

$$
\operatorname{Tf} f_{\text {Plant }}(\mathrm{s})=\frac{1}{s-0.2}
$$

A comparison with other controllers is shown in Figure 4.3.1.1. Applying the same adaptation gain, the output of the MRAC has a high overshoot and a long settling time. The VSMRC generates an offset error.

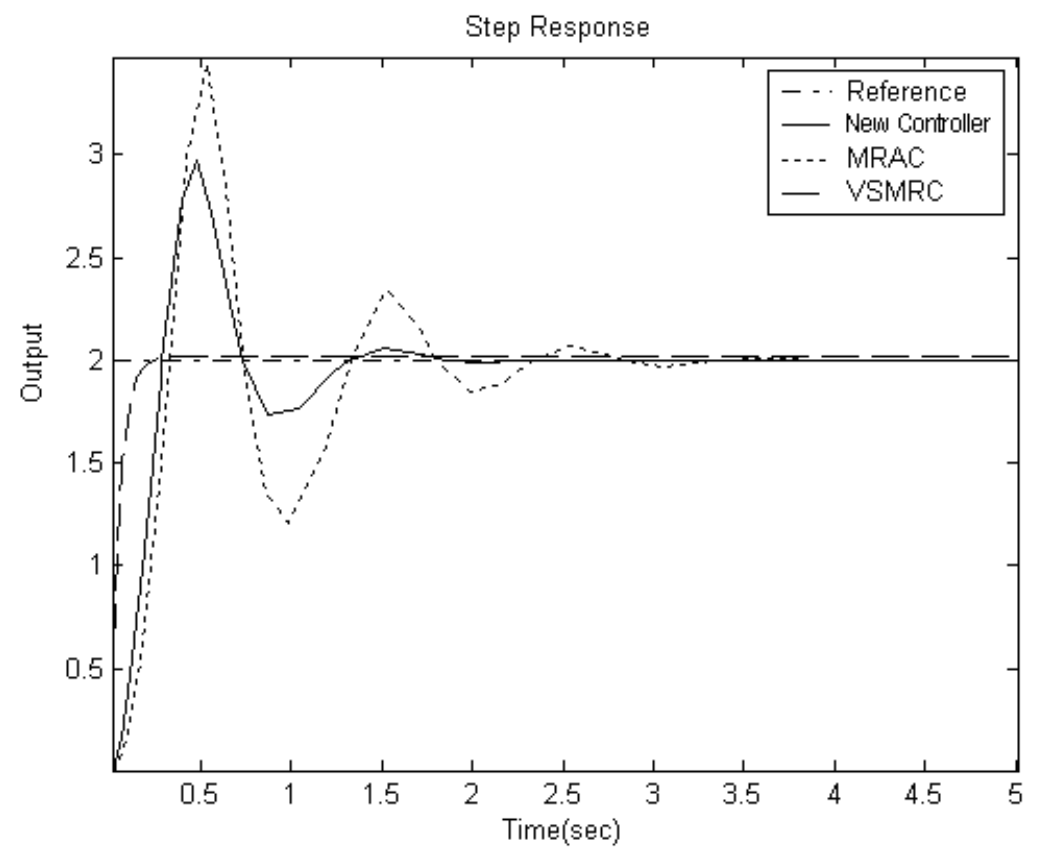

Figure 4.3.1.1, Regulation of an unstable system with a pole at $s=+0.2$. The results of MRAC and self sliding controller are shown.

Tracking: Consider a model with two poles at $s=-2, s=-3$ and one zero at $s=-1$ as 


$$
\operatorname{Tf}_{\text {Model }}(\mathrm{s})=\frac{s+1}{s^{2}+5 s+6}
$$

The control objective is to control the plant (4.3.1.1) to track the output of the model while excited with a sine wave of $1+5 \sin (5 t)$. Figure 4.3.1.2 shows the performance of the control and a comparison with those obtained from other controllers. MRAC generates a 50\% overshoot in the first peak whereas the new design with almost $10 \%$ overshoot in the first peak. After the adaptation is completed, the tracking performance at the same adaptation gain is demonstrated in Figure 4.3.1.3. As the figure shows, the new design provides a very close tracking profile and matches the reference. MRAC and VSMRC show the tracking with error.

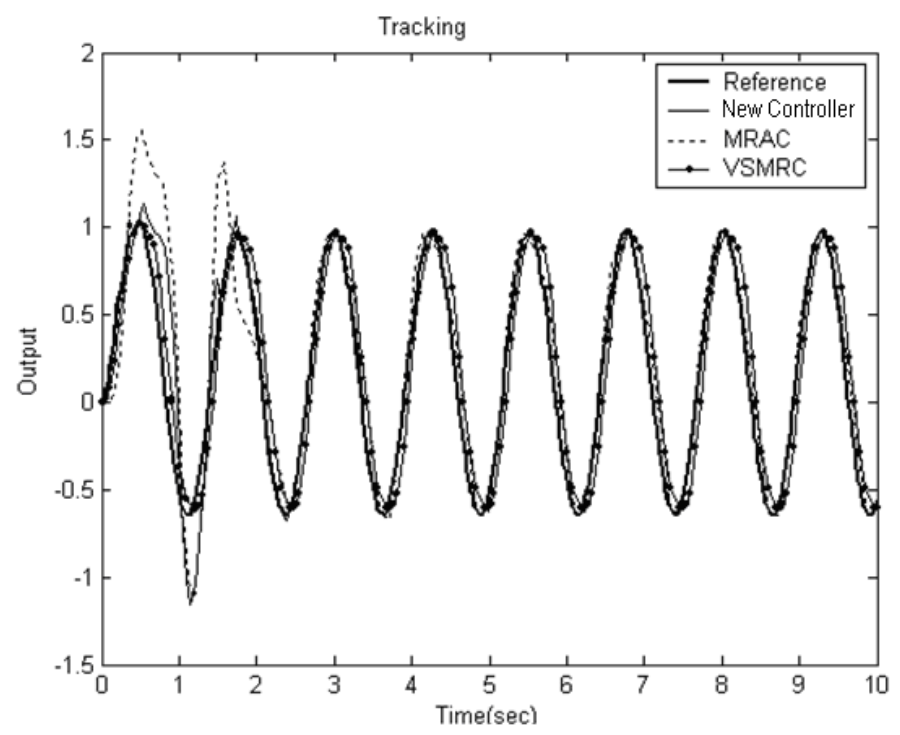

Figure 4.3.1.2, the self sliding controller and MRAC response to a constant and sine wave in frequency of $\omega=5(\mathrm{rad} / \mathrm{sec})$. 


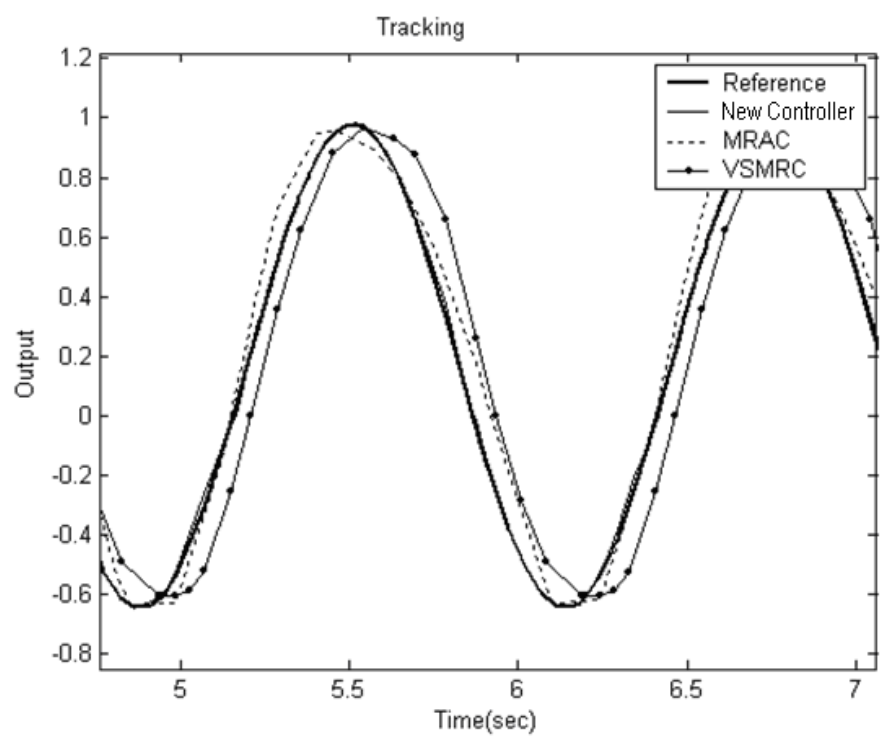

Figure 4.3.1.3, a close look at the tracking problem of Figure 6.

Double integrator: the control objective is to control the position of a double integrator system $G_{p}(s)=\frac{1}{s^{2}}$ according to a desired trajectory. Since the relative degree of the plant (twister) is grater than 1, the augmented error technique was used and the plant was initialized at initial condition 1 . The control law was the same as (4.2.1.3) with adaptation gains (4.2.1.4-6). The augmented error technique of (4.2.2.4) with adaptation of (4.2.2.5) was used. The tracking profile of a desired reference is shown in Figure 4.3.1.4.

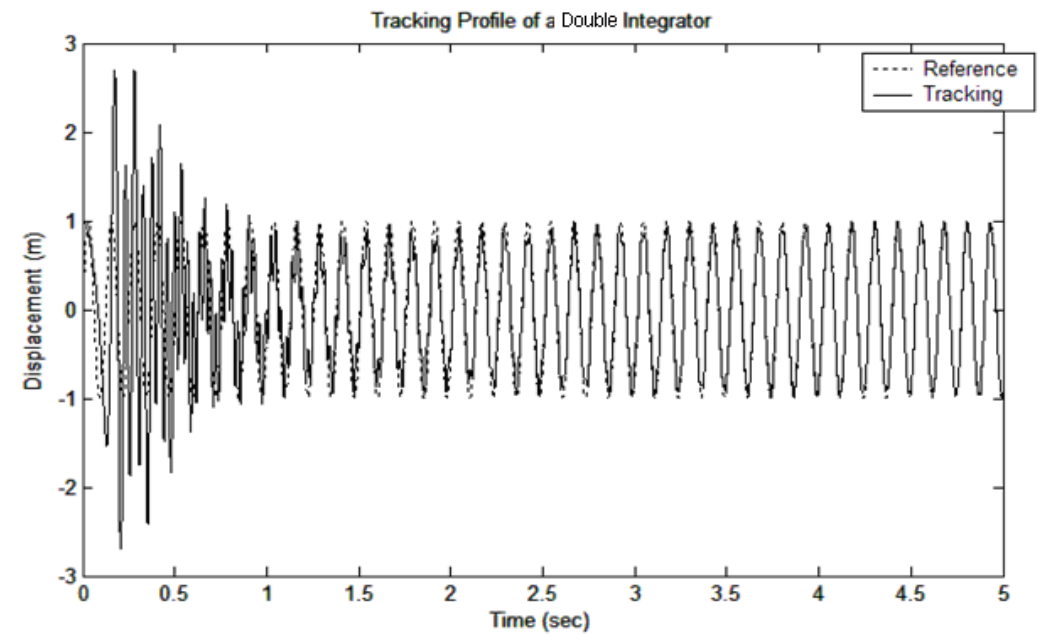

Figure 4.3.1.4, Tracking profile of a desired reference.

It was seen that the higher adaptation gain values resulted in shorter adaptation time. Figure 
4.3.1.5 shows the controller gain adaptation rates. As it is seen, the gains are saturated after almost 4 seconds and have just small variations to keep tracking of the desired reference.

The simple structure of the controller provides an easy design for a variety of systems with arbitrary relative degrees. The controller offers a design without the need for high order inputoutput signal filters and a simple control law independent from the system states.

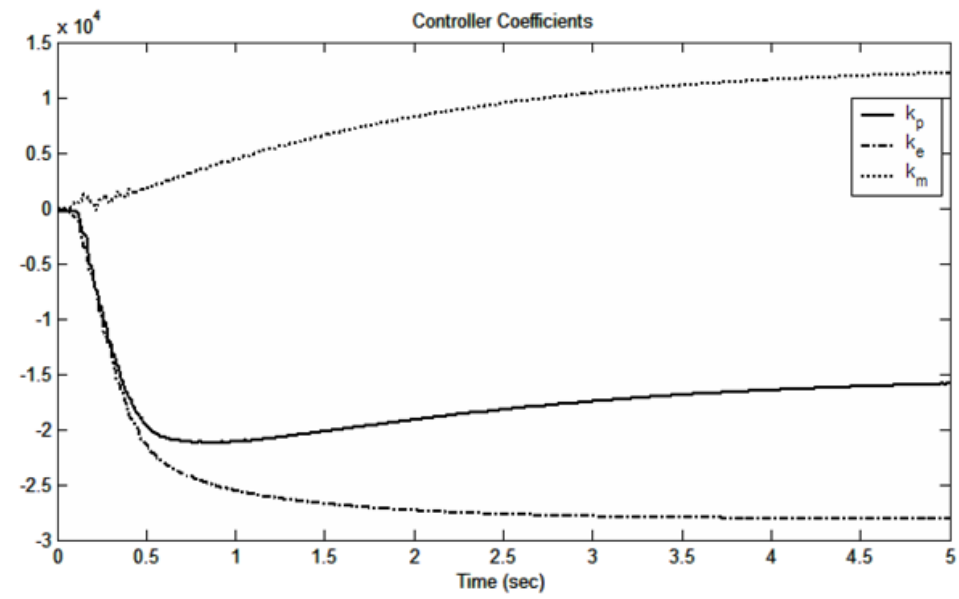

Figure 4.3.1.5, Gain adaptation of tracking control-double integrator

\subsection{Application in MEMS}

In this section, the controller is redesigned and applied for trajectory control of MEMS Lateral Comb Resonators. The controller is implemented in a real-time fast prototyping device (dSPACE PPC DS 1103). Simulations and experiment results are discussed in the following sections.

\subsubsection{Simulation Results}

Considering the MEMS device imperfect manufacturing steps, the parameter variation as a $5 \%$ additional mass on the actual device from its desired model is considered. The same mass variation is manufactured for the experimental results. Considering the second order structure of the LCRs, the complimentary augmentation error technique should be applied. Tracking performance of a complicated desired trajectory of 1 and $2.9 \mathrm{k}(\mathrm{rad} / \mathrm{sec})$ sine waves is shown in Figure 4.4.1.1. As the figure shows, the tracking waveform reaches the desired reference and follows the variations closely. Tracking error also reaches near zero values as the adaptation is completed in a short time. The control effort in this case is shown in Figure 4.4.1.2, where a comparison with reference input has shown a low energy-consuming controller. The controller can control the device with uncertain parameters in a short time and a low control effort. 


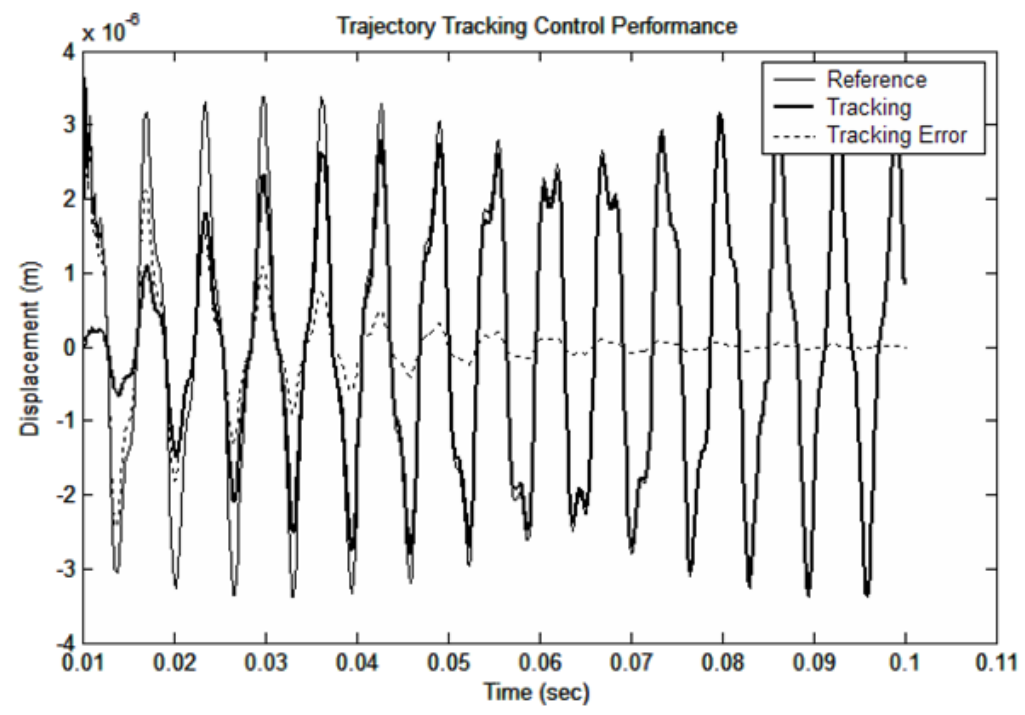

Fig. 4.4.1.1. Tracking performance of a desired reference. Tracking error reaches zero in a short time.

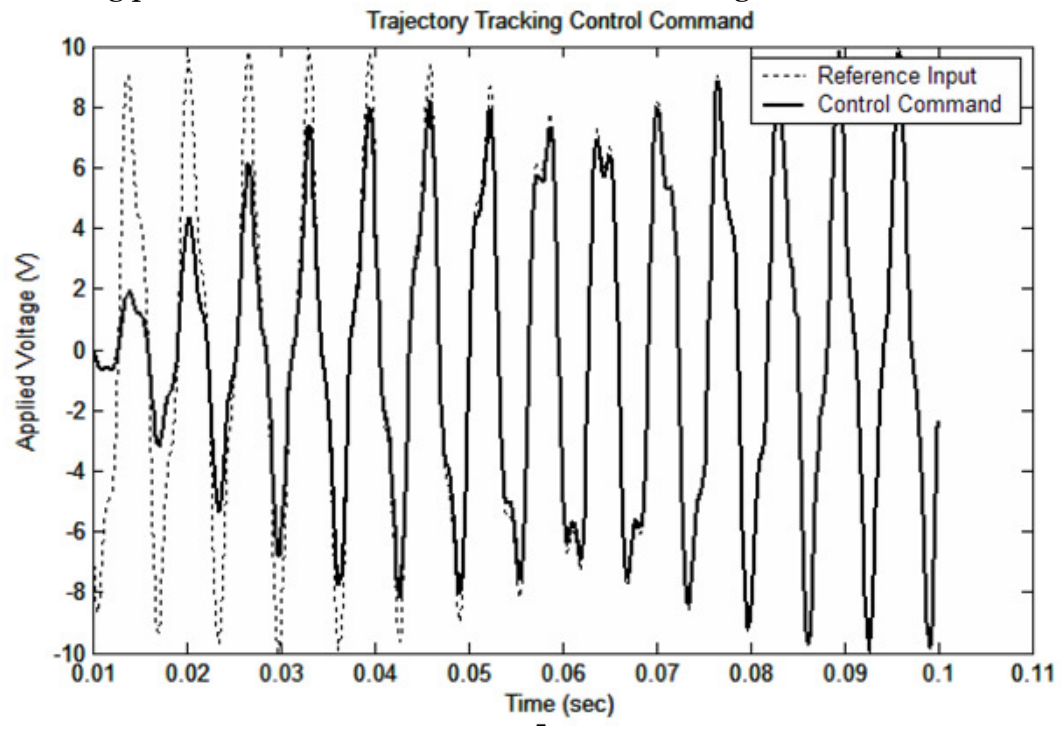

Fig. 4.4.1.2. Reference input and the control effort.

\subsubsection{Experimental Results}

In order to evaluate the performance of the controller in real-time applications, a Lateral Comb Resonator, shown in Figure 1.1, was designed and manufactured. The LCR contained 5\% additional mass on its shuttle. The device operated in free-air and contained other sources of uncertainties (due to manufacturing and environmental conditions) in its damping coefficient and spring constant values. The temperature and humidity as operating conditions affect the parameters of the device by causing stiction and increasing the stress in the system. The model of LCR had the parameter values as $m=2.2090 e-10, k_{s}=1.8906 e-2, \beta=6.7851 e-7$. 


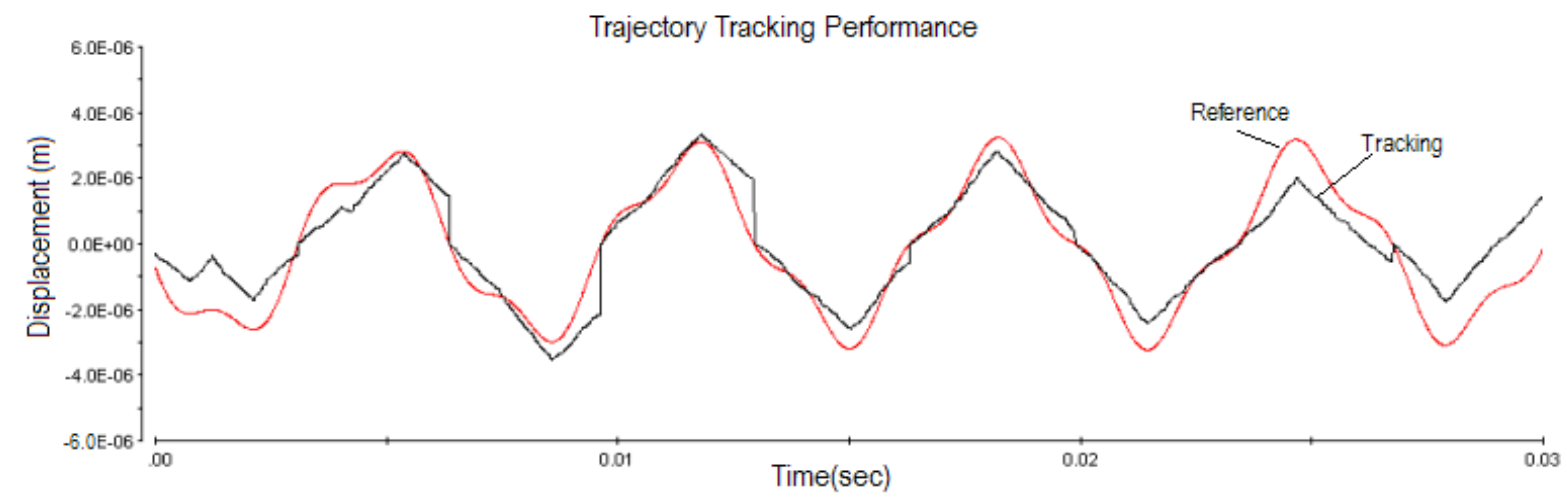

Fig. 4.4.2.1 Trajectory Tracking Performance, the desired and the tracking are shown.

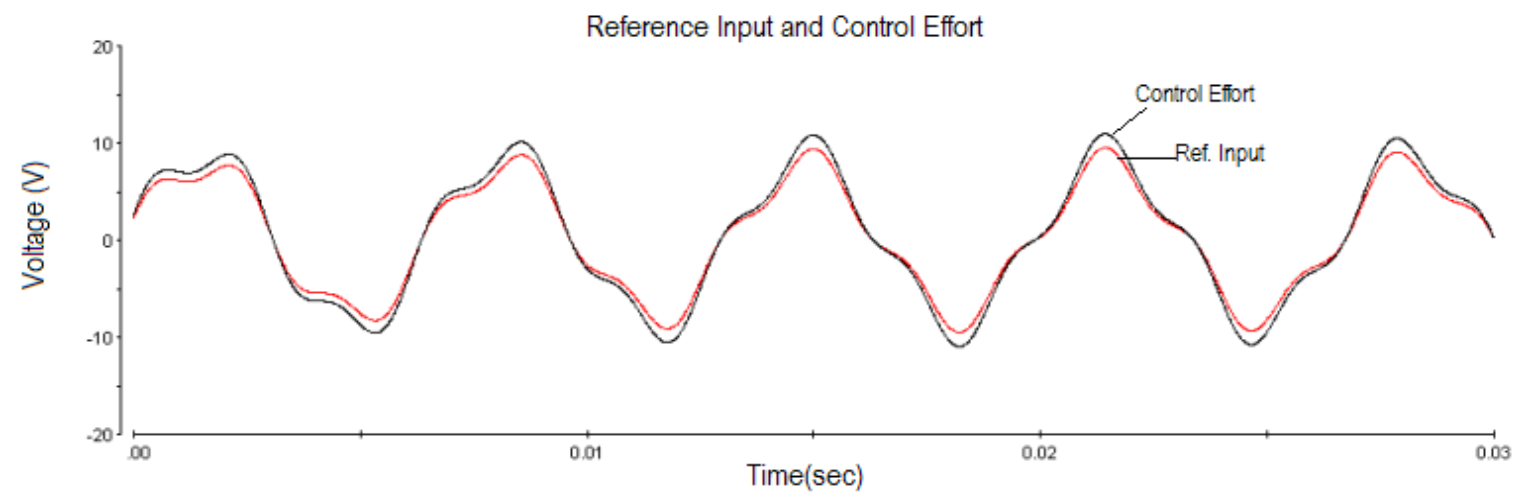

Fig. 4.4.2.2. The reference input and the controller effort.

The controller was implemented on a fast prototyping real-time control board, and was applied to the physical system. The successful demonstration of a trajectory tracking (as that of the simulation) is shown in Figure 4.4.2.1.

As the figure shows, the displacement-tracking waveform closely follows the desired reference, and validates the simulation results. According to the feedback technique resolution (Izadian, Wang, Dawson, Hornak \& Famouri. 2007) and existence of noise in the system, the tracking reaches to a bounded error. The control effort is shown in Figure 4.4.2.2. The controller generates a control command in the same level as that of the reference input. The control effort considering the imperfect device structure and parameters were different from the model, higher than those resulted from the simulation.

The experimental results have shown the capabilities of the controller in tracking control of a non-SPR system. The complimentary error augmentation technique provided a simple structure and a powerful tool for stabilizing the control system. The control law and gain adaptation techniques also enabled the controller for a filter-free design system. 


\subsection{Concluding Remarks}

A new controller was designed for tracking control of SPR systems and those with an arbitrary relative degree/higher orders. The gain adaptation techniques were designed according to the Lyapunov theory of stability. The controller did not require input-output filter designs and generated low control effort to control the uncertain parameter systems such as MEMS LCRs. The complimentary error augmentation technique was used for high relative degree systems to ensure the stability of tracking, enhance the robustness and bandwidth of the control system. The

controller performance was experimentally demonstrated in tracking control of a desired reference for MEMS Lateral Comb Resonators. 


\section{Chapter 5,}

\section{Intelligent Fault Diagnosis of MEMS LCR}

Fault diagnosis methods of MEMS lateral Comb Resonators are discussed in this chapter. First, some definitions on the fault diagnosis technique and then the application of Kalman filter are described. The application of parameter estimators in time varying parameter system is introduced in two different cases with a fixed forgetting factor value and adaptive forgetting factor adjustment. Fault diagnosis of each of these methods is described in simulations and real-time implementations.

\subsection{Definitions}

\subsubsection{Introduction}

Fault Tolerant controllers are those that can hold an acceptable performance of the system in case of fault occurrence in the system. They can do this by applying a suitable control command in each contingency in the system that requires fault diagnosis in the system and the control redesign. This section introduces the definition of the fault in systems and methods to identify and diagnose.

\subsubsection{Fault and Failure Definition}

In the beginning, some definitions are necessary on the difference between the fault and failure. A fault in a dynamical system is defined as a change in the construction of the system from the normal behavior. Different changes might show the same end effects in the system. A failure in the system occurs because of the severe changes in the normal operations of the system and the system becomes dysfunctional and irrecoverable. Parameter variations are the most common type of fault in microsystems. A faulty microsystem can still provide the expected operation by employing a proper control system as shown in Chapter 3 . 


\subsubsection{System Behavior}

Input-output sets define the systems behavior in case of normal or fault conditions. In a system, all sets of possible combination input-output pairs define the space $u \times y$. System behavior is a subset of this space denoted by $\beta$ in Figure 5.1.3.1. The output of the system due to an input might be placed in the accepted region of operation $A$ or as result of a change in the system might be inconsistent with the system dynamics and known as fault $C$. In this case, the system generates a different output for the same excitation.

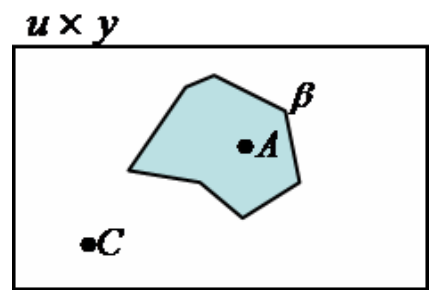

Figure 5.1.3.1. Input Output set. Healthy and non-healthy system output boundaries.

The system behavior is defined as $y(t)=k_{\text {sys }} u(t)$ where $k_{s y s}$ is known as the system. The set of possible real numbers which define the system input outputs are the I/O region, and a behavioral region is the possible outputs to the system defined as $\beta=\left\{(u, y): y=k_{s y s} u\right\}$. This shows which values of input and output belong together, and in case of any false output, the output set would belong to the other regions. Figure 5.1.3.2 show this matter clearly.

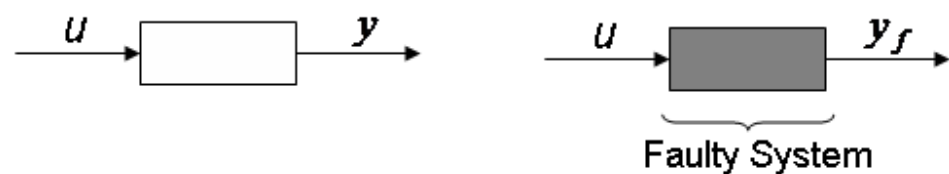

Figure. 5.1.3.2. Faultless and faulty system's response to the same input excitation.

If an input is applied to a faultless system, the possible pair of input-output is as sets of $A=\left(U, Y_{A}\right)$ and in case of faulty system, the same input might result in the output belong to the other region, known as $B=\left(U, Y_{B}\right)$. The faultless and fault regions might overlap at some areas as shown in Figure 5.1.3.3 such that a faulty output is placed to the shaded area. Faults are recognizable by these regions unless they overlap and belong to two regions like point B in Figure $5 \cdot 1 \cdot 3 \cdot 3$. 


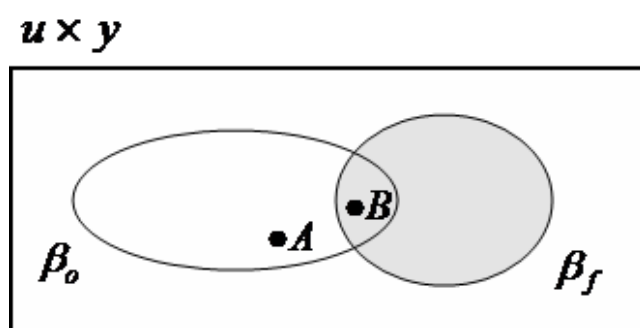

Figure 5.1.3.3. Faultless and Faulty systems output boundaries. $\beta_{0}$ is the faultless system's output region.

\subsubsection{Fault and Model Uncertainty}

In a second order differential equation, for instance, an additional external signal to the system or a parameter deviation from the desired value can be identified as fault. If a signal is added to the input of the system, this can be identified as the additive fault and in case of unknown parameters, the system might have multiplicative fault. Faults are changes that should be detected and their results be removed by a proper action. Model uncertainties and disturbances are those that their effects should be removed by filters or controls.

\subsubsection{Faulty System Modes and Conditions}

A safe system is the one without any harm or under any harmful tension. If the safety is provided by a controller it is called a safe-to-fail system. Reliable system is the one that provides the required functions in a period under normal conditions. Fault tolerant systems cannot change the reliability of the component but they can enhance the overall reliability of the system by keeping the overall system operational. Fault tolerant system is a system in which a single fault does not develop to a failure of the closed loop system by keeping the performance the same after the fault in the worst case and if so, the system is fault-operational. Fault and recovery processes put the system in different regions of performance. As Figure 5.1.5.1 shows, the safe operation of the device is separated from the dangerous region of operation. The safe operation zone is divided into degraded and unacceptable performance operation zones. Fault and recovery change the performance of the operation and put the system into the degraded and required performance regions (Blanke, Kinnaret, Lunze \& Staroswiecki 2006). 


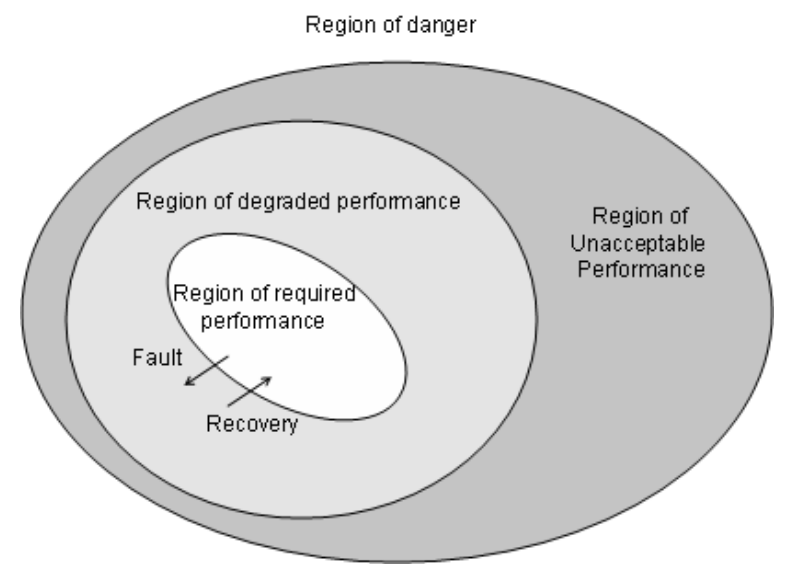

Figure 5.1.5.1. Performance categorizing of different system behavior (Blanke, Kinnaret, Lunze \& Staroswiecki 2006).

\subsubsection{Fault Diagnosis:}

Fault tolerant controller contains two main parts as fault diagnostics blocks and controller redesign. Diagnostic block makes use of the input-output sets and tests their belonging to the plant model. The results of this part are used for the controller redesign part. In case of a fault in the system the fault diagnosis unit identifies the fault and makes the control loop fault tolerant. Some controllers such as Robust controllers and Adaptive controllers provide active fault tolerant controllers. The fault tolerant control properties are observed mainly in systems with slowly varying parameters.

If a dynamical system generates pairs of $u-y$ and is subject to the fault $f$, the system behavior depends on the type of fault or $f \in F$, where $f_{o}$ shows a faultless system. The problem is to identify the fault over a given set of input-output pairs $(\mathrm{I} / \mathrm{O}) \rightarrow(\mathrm{U}, \mathrm{Y})$ within a time zone as

$$
\begin{aligned}
& \mathrm{U}=\left(\mathrm{u}(0), \mathrm{u}(1), \ldots, \mathrm{u}\left(\mathrm{k}_{\mathrm{h}}\right)\right) \\
& \mathrm{Y}=\left(\mathrm{y}(0), \mathrm{y}(1), \ldots, \mathrm{y}\left(\mathrm{k}_{\mathrm{h}}\right)\right)
\end{aligned}
$$

Online fault diagnostic requires the information resulted from a dynamical model and in time series of the signals and no physical inspection should be done to identify the fault. In control systems, the location and magnitude of the fault is very important and have to be identified through the fault diagnosis process. There are different approaches for fault diagnosis which are mainly named as followings:

- Consistency based diagnosis

- Diagnosis of Continuous variable systems 
The idea of consistency based diagnosis returns to the fact that for fault diagnosis the information of input-output $(\mathrm{U}, \mathrm{Y})$ is not enough. A reference should exist to define the normal behavior of the plant. The reference is determined by the possible sets of I/O for a known system which represents the behavior of the system. Considering the current I/O pair (U,Y) in Figure 5.1.3.1 at point $A$ which for a correct model it belongs to set B and if the system is faulty it

generates $\hat{\mathrm{Y}}$ as $(\mathrm{U}, \hat{\mathrm{Y}})$ which belongs to the region $\mathrm{C}$, and if so the fault is detectable. The principle of this type of fault diagnosis is comparing the performance of the current I/O pairs with faultless system behavior B, it checks the consistency of the current pairs with the nominal system behavior. In this case, fault makes the output behavior inconsistent except in same behavior of the system in case of overlapping areas of healthy and faulty systems. In general, a system should be diagnosable and fault detectable to avoid such uncertainties of decisions.

As a conclusion:

- Fault is occurred if: $(\mathrm{U}, \mathrm{Y}) \notin \mathrm{B}_{0}$

$\circ$ And the fault is identified and isolated if: $(U, Y) \in B_{f}$

\subsection{MEMS Fault Diagnosis Using Kalman Filters}

Micro Electro Mechanical Systems are important parts of many advanced applications ranging from satellite stabilizers to commercial accelerometers. A basic structure of MEMS Lateral Comb Resonators consists of two sets of fixed combs on both sides of a moving stage. The shuttle (moving part) has a comb-shape structure with fingers placed in the space between those of the fixed structure to overlap initially. The shuttle is suspended on two sets of springs and can be laterally displaced by electrostatic force generated between the overlapped fingers. Fabrication process of MEMS involves several steps that introduce parameter uncertainties in model and parameters of the system. Different techniques are developed to measure the infant mortality rate of microfabrication processes. Correcting these processes requires a model matching technique to allocate a predefined model to the operating system. This allows instantaneous model verification and can be applied to control of these systems in constantly changing conditions. In addition, this supervisory unit can be used to monitor in-operation devices for assured high quality operations, and diagnose any misbehavior in the device instantly.

The Lateral Comb Resonators can be modeled in a second order mass-spring-damper system. To model the parameter variations due to the manufacturing, in-operation wearing of the parts and fault, the coefficients of dynamical equation are considered as time varying values. Structural variations can also be modeled as a shift in the values of these parameters. The device studied in this section, is considered to operate in open air conditions to simulate the loss of vacuum conditions. In addition, manufacturing processes of the device and instant shocks may increase the risk of spring constant variations. 
Faults can be modeled by recently developed tool such as Contamination and Reliability Analysis of Micro-Electro Mechanical Layout (CARAMEL) which interprets system structural variations in form of electric circuit elements (Kolpekwar, Kellen, Blanton. 1999) (Kolpekwar, Blanton. 1997). In this technique, suitable models containing different fault possibilities can be obtained and used as residual signal generators. Kolpekwar et. al, have modeled up to 4000 types of structural defects by CARAMEL (Kolpekwar, Blanton. 1997). However, defects such as change of environmental and operating environment e.g. satellites, fluid control devices and micropumps, in which microsystem is under influence of radiation, rapid environmental changes and noise (Schanwald, Schwank, Sniegowski, Walsh, Smith, Peterson, Shaneyfelt, Winkour, Smith, Doyle. 1998), cannot be modeled by CARAMEL. Since, in this section, the displacement of MEMS is monitored by a laser beam and is recovered from recorded optical waveforms, conventional modeling tools based on structural defects would not accurately model the dynamics of the system. Therefore, a robust modeling technique against noise in the system and compatible with optical monitoring method is required.

In this section, Kalman filters are utilized to model MEMS devices to accurately factor in the system and measurement noise resulted from the hybrid optical measurement technique (used in the displacement monitoring technique). The structure of Multiple-Model Adaptive Estimators is illustrated and their application to LCRs is simulated and experimentally proven for fault diagnosis purposes. The effect of noise in decision-making process is studied for over and underestimated noise in LCR systems. LCRs with different parameters are designed and fabricated for practical implementation of MMAE technique to demonstrate successful fault diagnosis in applications.

\subsubsection{Lateral Comb Resonator Modeling}

A quick reminder from chapter 1 on the structure of micro comb resonators structure and governing equations are presented in this part. A typical Lateral Comb Resonator consists of a moving stage also known as shuttle, stationary combs and suspension springs on both sides (Fig. 5.2.1.1.a). The LCR can be modeled as a mass spring damper (Fig. 5.2.1.1.b) in a linear second order differential equation. Electrostatic force is used to move the shuttle by applying two identical DC voltages onto the stationary combs at both sides of the LCR. A time-varying voltage (typically a sine wave) is applied to the shuttle to provide enough force for the device to follow a certain trajectory and perform a specific task. The ideal electrostatic force depicted in (5.2.1) is a function of applied voltages and geometry of the device, however, there are many other phenomena that affect the force value that are not considered in this electrostatic force equation (Schanwald, Schwank, Sniegowski, Walsh, Smith, Peterson, Shaneyfelt, Winkour, Smith, Doyle. 1998). The ideal force is 


$$
F_{e}=-4 n \varepsilon \frac{t}{g}\left(V_{b} . V_{s}\right)
$$

where $F_{e}$ is the electrostatic force, $n$ is the number of fingers on one side of the device, $\varepsilon$ is the permittivity of air, $t$ is the length of a finger, $g$ is the gap between fingers, $V_{b}, V_{s}$ are applied stator and shuttle voltages respectively. Furthermore, there are parasitic forces originated from imperfect manufacturing steps and stiction, which is caused by trapped surface charges, capillary forces and surface roughness such that they distorts the electric field between very small plates. Environmental conditions such as humidity influence the stiction of the parts. Therefore, the force generated is an uncertain quantity of uncertain parameters in addition to unknown variables. A simple mass-spring-damper model for the system can be written as

$$
F_{e}=m \ddot{y}+\beta \dot{y}+2 k_{s} y+F_{d},
$$

where $m$ is the mass of the center comb (shuttle), $y$ is the displacement, $k_{s}$ is the spring constant of one side, $\beta$ is the damping coefficient, $F_{d}$ is the force due to the load. In driving of microstructure applications (such as connecting beam to rotating gears), the load can be applied directly to the shuttle. As mentioned earlier, device parameters are affected by manufacturing processes and operating and environmental conditions. Under these conditions, the model containing uncertainties can be expressed as

$$
\hat{F}_{e}=\hat{m} \ddot{y}+\hat{\beta} \dot{y}+2 \hat{k}_{s} y+\hat{F}_{d},
$$

where $\left({ }^{\wedge}\right)$ denotes uncertain values of the associated parameters. The fact is that the desired system should operate similar to (5.2.2) whereas the manufactured device which behaves differently like (5.2.3). More description on modeling, control, and displacement monitoring techniques for a LCR are introduced in (Izadian, Hornak \& Famouri. 2006), (Wang, Dawson, Hornak, Famouri \& R. Ghaffarian. 2004), (Dawson, Wang, Chen, Famouri \& Hornak. 2000), (Dawson, Chen, Brown, Famouri \& Hornak. 2000), (Park, Horowitz \& Tan. 2001), ( Wang. 2005), (Shkel, Horowitz, Seshia, Park \& Howe. 1999), (Horowitz, Cheung \& Howe. — ), (Fedder \& Howe. 1996), (Tang, Lim \& Howe. 1992), (Dawson, Wang, Chen, Famouri \& Hornak. 2000), (Wang, Dawson, Chen, Famouri \& Hornak. 2000),(Bhansali, Zhang, Zmood, Jones, \& Sood. 2000), (Li \& Horowitz. 2001), (Pannu, Chang, Muller \& Pisano. 2000). This system is represented in the state space form as 


$$
\begin{aligned}
& \left\{\begin{array}{l}
\dot{x}_{1}=x_{2} \\
\dot{x}_{2}=\frac{1}{\hat{m}}\left(-2 \hat{k}_{s} x_{1}-\hat{\beta} x_{2}\right)+\frac{\hat{F}_{e}-\hat{F}_{d}}{\hat{m}} .
\end{array}\right. \\
& y=\left[\begin{array}{ll}
1 & 0 \\
0 & 1
\end{array}\right] x
\end{aligned}
$$

\subsubsection{Fault In Lateral Comb Resonators}

As illustrated in previous section, a second order mass-spring-damper system is used to model the lateral comb resonator. The parameters of this system are considered variable to model different types of fault occurring during the operation. Parametric variation and those structural variations that do not result in a failure in the device are investigated in this paper. Fault in microsystems can be originated from local defects, parameter tolerance, design problem, operation and/or system level defects (Rosing, Richardson, Dorey \& Peyton. 1999). Faults might happen by braking of different parts of the device such as suspension springs or fingers (in lateral comb resonator). In any of these cases, asymmetries will cause behavioral variations and an unwanted deviation from the desired output.

In open air applications, there is a chance of falling dust and particles on the structure of the device which may cause a mass change. In addition, the silicon made structures absorb the humidity of air which causes mechanical properties of suspension springs change. Large conductive particles fallen on areas between the fingers of shuttle and fixed structure might cause a short circuit in the device, join the parts together and result in a failure.

For the devices operating in vacuum conditions damage in the container might result in the loss of vacuum and consequently change of viscosity.

A fault scenario is developed in the next sections which covers parameter variations used in MMAE and fault diagnosis applications. MEMS comb resonators are also fabricated with different parameters to experimentally examine the diagnostic capabilities of the proposed application.

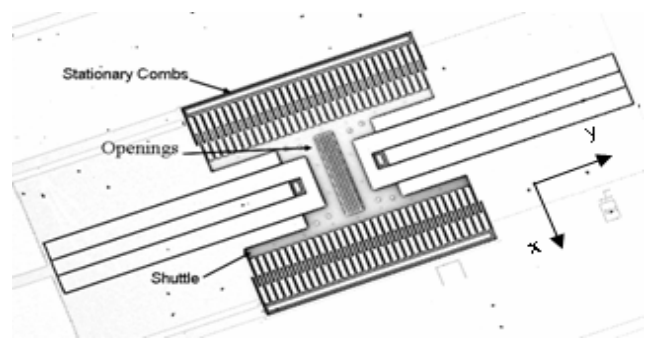

(a) 


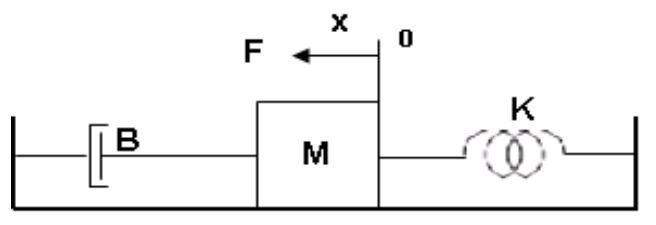

(b)

Fig. 5.2.1.1, a) Lateral Comb Resonator manufactured by WVU, b) Mass-Spring-Damper, schematic model of Lateral Comb Resonator.

\subsubsection{Theory Development}

\subsubsection{Kalman Filter Design for LCRs}

Consider the steady state Kalman filter model of a Lateral Comb Resonator associated with different types of fault conditions denoted by subscript $k$ as

$$
\begin{aligned}
& x_{k}\left(t_{i}\right)=\Phi_{k} x_{x}\left(t_{i-1}\right)+\Gamma_{k} u\left(t_{i-1}\right)+G_{k} w_{k}\left(t_{i}\right) \\
& z_{k}\left(t_{i}\right)=H_{k} x_{k}\left(t_{i}\right)+v_{k}\left(t_{i}\right)
\end{aligned}
$$

where:

$x_{k}$ is Kalman filter model state space vector

$\Phi_{k}$ is system matrix of Kalman filtering model

$\Gamma_{k}$ is input matrix of Kalman filter

$u$ is input vector

$G_{k}$ is Kalman filter model noise input matrix

$w_{k}$ is white discrete-time noise dynamics in the input of Kalman filter with zero mean and known variance as

$$
E\left\{w_{k}\left(t_{i}\right) w_{k}^{T}\left(t_{j}\right)\right\}=\left\{\begin{array}{l}
Q_{k}, t_{i}=t_{j} \\
0, t_{i} \neq t_{j}
\end{array} .\right.
$$

$z_{k}$ is the measurement vector of the Kalman filter model

$H_{k}$ is the Kalman filter model output matrix

$v_{k}\left(t_{i}\right)$ is the white discrete-time measurement noise in the output of the Kalman filter model with zero mean, independent from $w_{k}$ as following: 


$$
E\left\{v_{k}\left(t_{i}\right) v_{k}^{T}\left(t_{j}\right)\right\}=\left\{\begin{array}{l}
R_{k}, t_{i}=t_{j} \\
0, t_{i} \neq t_{j}
\end{array}\right.
$$

Modeling of the system with Kalman filters, in the same order as that of the true (healthy) system, takes both structure and system level noise into account, which is considered a great advantage over the CARAMEL-modeling tool. Having the model outputs compared with the actual output of microsystem, generates the residual signals for fault-representative models in each time interval. The residuals are then evaluated in the hypotheses-testing algorithm to extract the system parameter variation and weight allocations associated with each residual.

Let consider Kalman filtering model representation as

$$
\hat{x}_{k}\left(t_{i}\right)=\Phi_{k} \hat{x}_{x}\left(t_{i-1}\right)+\Gamma_{k} u\left(t_{i-1}\right)+K_{e}\left(y_{k}\left(t_{i}\right)-H_{k} \hat{x}_{k}\left(t_{i}\right)\right),
$$

where $\hat{x}_{k}$ is the estimation of state space variable, $y_{k}$ is actual output expected from the model, and $K_{e}$ is Kalman filter gain recursively obtained from the following procedure

$$
K_{e}(n)=\Phi_{k} P(n) H_{k}^{T}\left[R+H_{k} P(n) H_{k}^{T}\right]^{-1} \text { For } n=1,2 \ldots
$$

where $P$ is covariance matrix and is updated using

$$
P(n+1)=G_{k} Q G_{k}^{T}+\Phi_{k} P(n) \Phi_{k}^{T}-\Phi_{k} P(n) H_{k}\left[R+H_{k} P(n) H_{k}^{T}\right]^{-1} H_{k} P(n) \Phi_{k}^{T}
$$

This value updates the Kalman gain for each iteration. The residual signal is the difference between the output of Kalman filter and that of the actual system. For each of the models there is a residual signal defined and used to compute conditional probability density function. Residuals are computed as

$$
\left.r_{k}\left(t_{i}\right)=z\left(t_{i}\right)-H_{k} \hat{x}_{k}\left(t_{i}\right)\right) .
$$

Equation (5.2.3.1.7) explains the differences between the measurements $(z)$ and the Kalman estimated outputs.

\subsubsection{Multiple-Model Adaptive Estimation Equations (HANLON \& MAYBECK. 200o)}

The hypothesis-testing algorithm continuously monitors the residual signal variations. If the output of any of Kalman filters match the output of the system, the mean of residual signal 
becomes zero and therefore the covariance of this residual is computed. Hence, the Kalman filter generated residual signal, in this case, contains a zero mean white Gaussian sequence with covariance computable by

$$
\psi_{k}=C_{k} P_{k} C_{k}^{T},
$$

which results in conditional density function of the output measurement $z\left(t_{i}\right)$ of the system for $\mathrm{k}^{\text {th }}$ Kalman filter over the measurement history of $Z\left(t_{i-1}\right)=\left[z^{T}\left(t_{1}\right) \ldots z^{T}\left(t_{i-1}\right)\right]$. The conditional density is therefore written as

$$
f_{z\left(t_{i}\right) \mid h, Z\left(t_{i-1}\right)}\left(z_{i} \mid h_{k}, Z_{i-1}\right)=\beta_{k} \exp \{\bullet\}
$$

with

$$
\beta_{k}=\frac{1}{(2 \pi)^{l / 2}\left|\psi_{k}\right|^{l / 2}},
$$

and

$$
\{\bullet\}=\left\{-\frac{1}{2} r_{k}^{T}\left(t_{i}\right) \psi_{k}^{-1} r_{k}\left(t_{i}\right)\right\}
$$

The conditional probability hypothesis for the $\mathrm{k}^{\text {th }}$ system is defined as

$$
p_{k}\left(t_{i}\right)=\operatorname{Pr}\left\{h=h_{k} \mid Z\left(t_{i}\right)=Z_{i}\right\} .
$$

This value can also be computed as

$$
p_{k}\left(t_{i}\right)=\frac{f_{z\left(t_{i}\right) \mid h, Z\left(t_{i-1}\right)}\left(z_{i} \mid h_{k}, Z_{i-1}\right) \cdot p_{k}\left(t_{i-1}\right)}{\sum_{j=1}^{K} f_{z\left(t_{i}\right) \mid h, Z\left(t_{i-1}\right)}\left(z_{i} \mid h_{j}, Z_{i-1}\right) \cdot p_{j}\left(t_{i-1}\right)} .
$$

The conditional probability density function requires a priori samples to compute the current values and is normalized over the complete sum of the conditional probabilities of all systems(Park, Horowitz \& Tan. 2001). The largest conditional probability among all can be used as an indicator of fault in the systems (note that each fault-representing system should be modeled separately). In addition, they can weight the outputs of the systems and define the weighted output of each fault-model. In some applications, probabilities change rapidly and make output of the system unpredictable then the output should be compared with a threshold. 


\subsubsection{Simulation and Experimental Results}

\subsubsection{Simulation and discussion:}

To investigate the application of MMAE in fault diagnosis of MEMS devices, a fault scenario consist of typical parameter variations is designed in seven steps as:

1. Healthy operation for $2 \mathrm{k}$ samples

2. $\quad+5 \%$ mass variation for $1 \mathrm{k}$ samples

3. $+10 \%$ mass variation for $1 \mathrm{k}$ samples

4. $\quad+5 \%$ spring constant variation for $1 \mathrm{k}$ samples

5. $\quad+10 \%$ spring constant variation for $1 \mathrm{k}$ samples

6. $+30 \%$ change in damping coefficient for $1 \mathrm{k}$ samples

7. Return to normal condition after $2 \mathrm{k}$ samples.

These series of events occur consecutively and cover common types of variations that microsystems undergo during operation. The displacement variation in +5 and $+10 \%$ change of the mass of LCR are shown in Figure (Figure 5.2.4.1.1). The displacement resulted from the spring constant variations are shown in Figure (Figure 5.2.4.1.2). Any change in spring constant values causes higher displacement variations. Therefore, faults resulting from the spring constant variations are more probable to be diagnosed.

Damping coefficient variations cause a delay from the original waveform. Figure 5.2.4.1.3 shows the phase shift resulted by a 30\% change of damping coefficient and matches step 6 of the fault scenario.

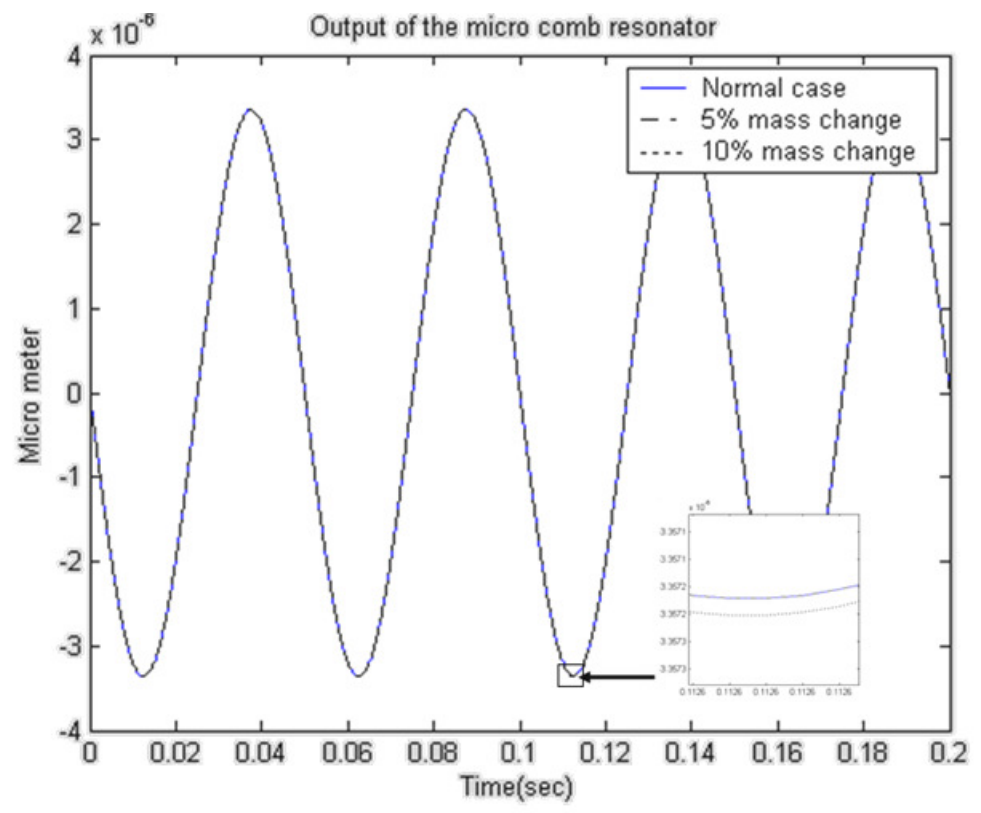

Fig. 5.2.4.1.1 Mass change effects on the system output. All the outputs are very close since the total mass is negligible itself. 


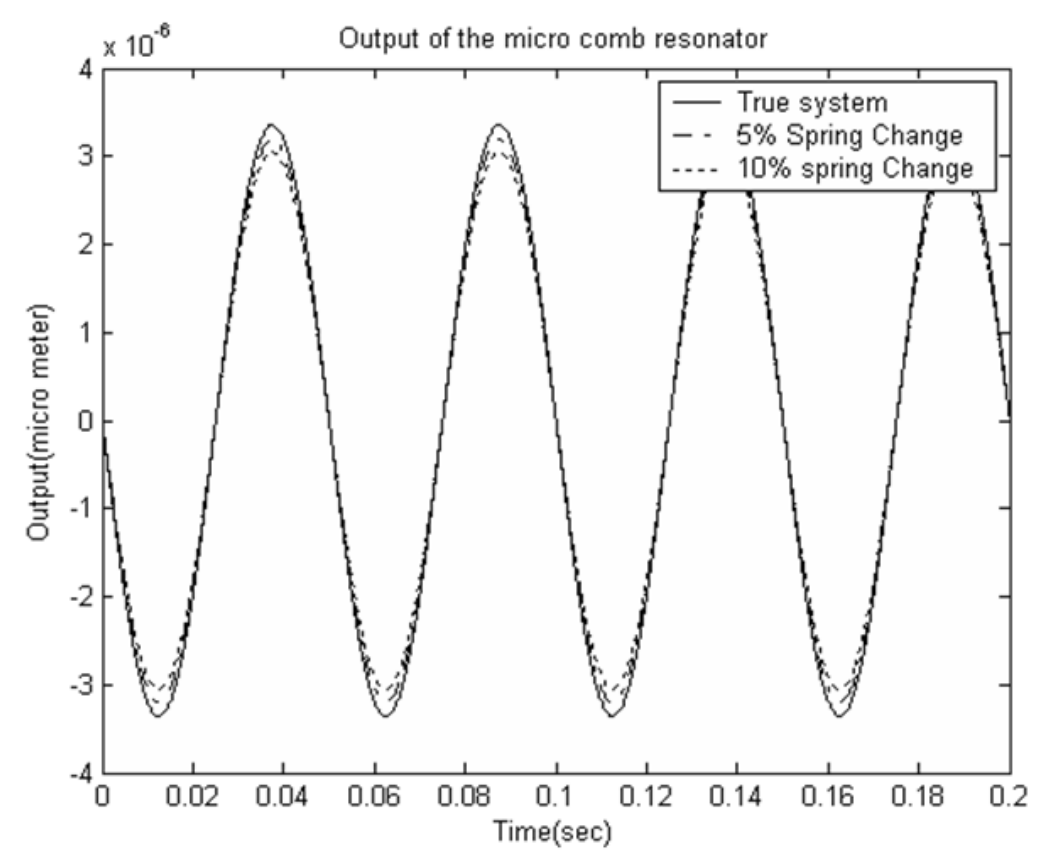

Fig. 5.2.4.1.2, Output of the system in case of the spring constant change to 5 and $10 \%$.

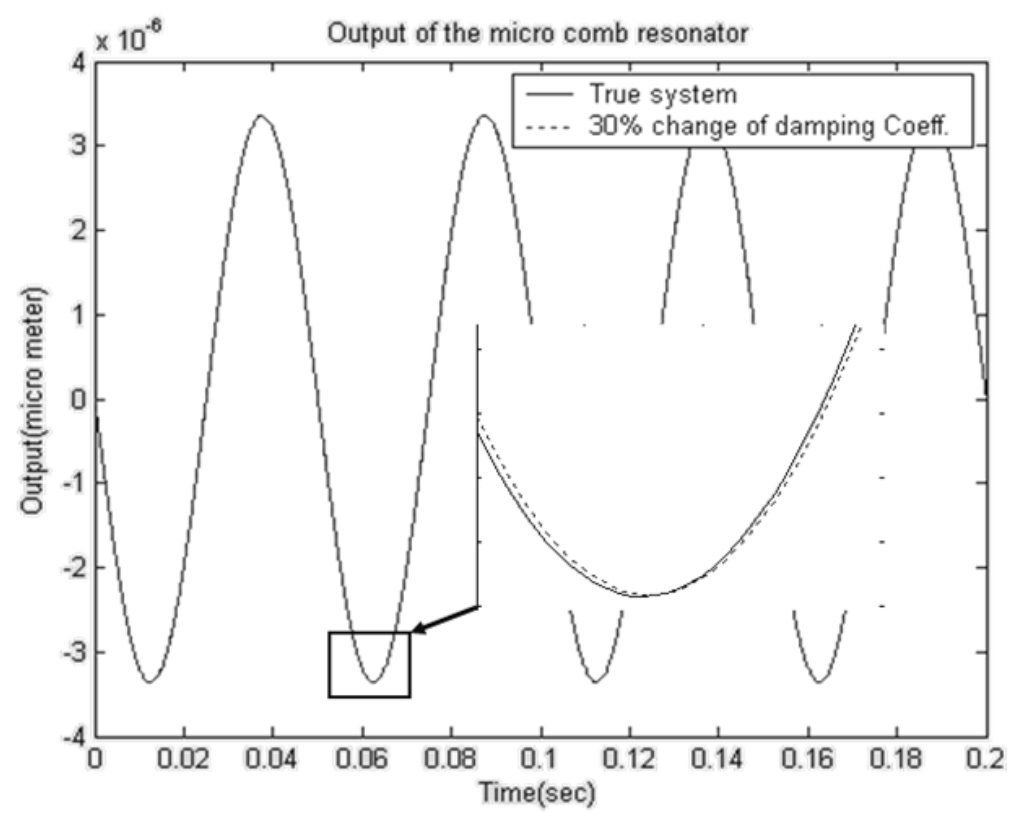

Fig. 5.2.4.1.3, The effect of the spring constant change on the Lateral Comb Resonator's output, 30 percent change from the true system output.

The noise content in microsystem has significant effect on the conditional probability functions. The effect of noise in fault diagnosis is studied in two cases of noise under and overestimation in Kalman filter designs. The system contains a zero mean white noise with $\mathrm{Q}=1 \mathrm{e}-4$, $\mathrm{R}=1 \mathrm{e}-4$ variances in the measurement signal and system respectively. Considering the noise 
content in the system, Kalman gains are computed in each of these models and are shown in Table 5.2.4.1.1.

Table 5.2.4.1.1, Kalman filter gains of the system in different cases as mentioned in fault scenario

\begin{tabular}{|c|c|c|c|c|c|}
\hline (Health system) & (5\% Mass) & (10\% mass) & (5\% Spring cons) & (10\% spring cons.) & (30\%damp.coeff.) \\
\hline $1.0 \mathrm{e}-006 *$ & $1.0 \mathrm{e}-006 *$ & $1.0 \mathrm{e}-005^{*}$ & $1.0 \mathrm{e}-006 *$ & $1.0 \mathrm{e}-008 *$ & $1.0 \mathrm{e}-008 *$ \\
\hline $\begin{array}{lll}-0.0002 & -0.4242\end{array}$ & -0.0000 & $0.0000-0.0000$ & -0.0000 & -0.0022 & 0.0000 \\
\hline $0.0002 \quad 0.4242$ & -0.3824 & $-0.1116 \quad 0.1116$ & -0.3265 & -0.5752 & $0.3054 \quad-0.3054$ \\
\hline
\end{tabular}

Running the probability density functions over the steps in fault scenario designates a weight between $\mathrm{o}$ and 1 to each system in each time instant. Higher probability numbers indicate the validity of that particular system. Figure 5.2.4.1.4 shows the weights computed by probability density functions in evaluation of the residual signal in the case of normal noise content in microsystem. As the figure shows, the highest weight is allocated to the proper system (shown by similar numbers) which matches the same scenario instants.

As Figure 5.2.4.1.4 shows, in each step (also labeled according to the fault sequence) the transition from one step to the next is quick. The fault diagnostic unit accurately identifies the faults as predetermined in the fault scenario.

In case the noise is overestimated in the Kalman gain computations, the fault diagnostic unit identifies the fault rapidly from one type to another i.e, the transition from mass change to spring constant requires a short time. However, in the same category of fault diagnosis (from 5 to $10 \%$ variations) the noise results in delay in fault recognition which is also shown in Figure 5.2.4.1.5. A $35 \%$ noise overestimation influences the transitions between 5 and $10 \%$ change in mass and spring constants, but still provides a quick switching from mass to spring constant transitions. As the figure shows, delays in transitions from steps 2 to 3 and steps 4 to 5 were observed. Existence of noise masks negligible parameter variations that are required to be diagnosed. 


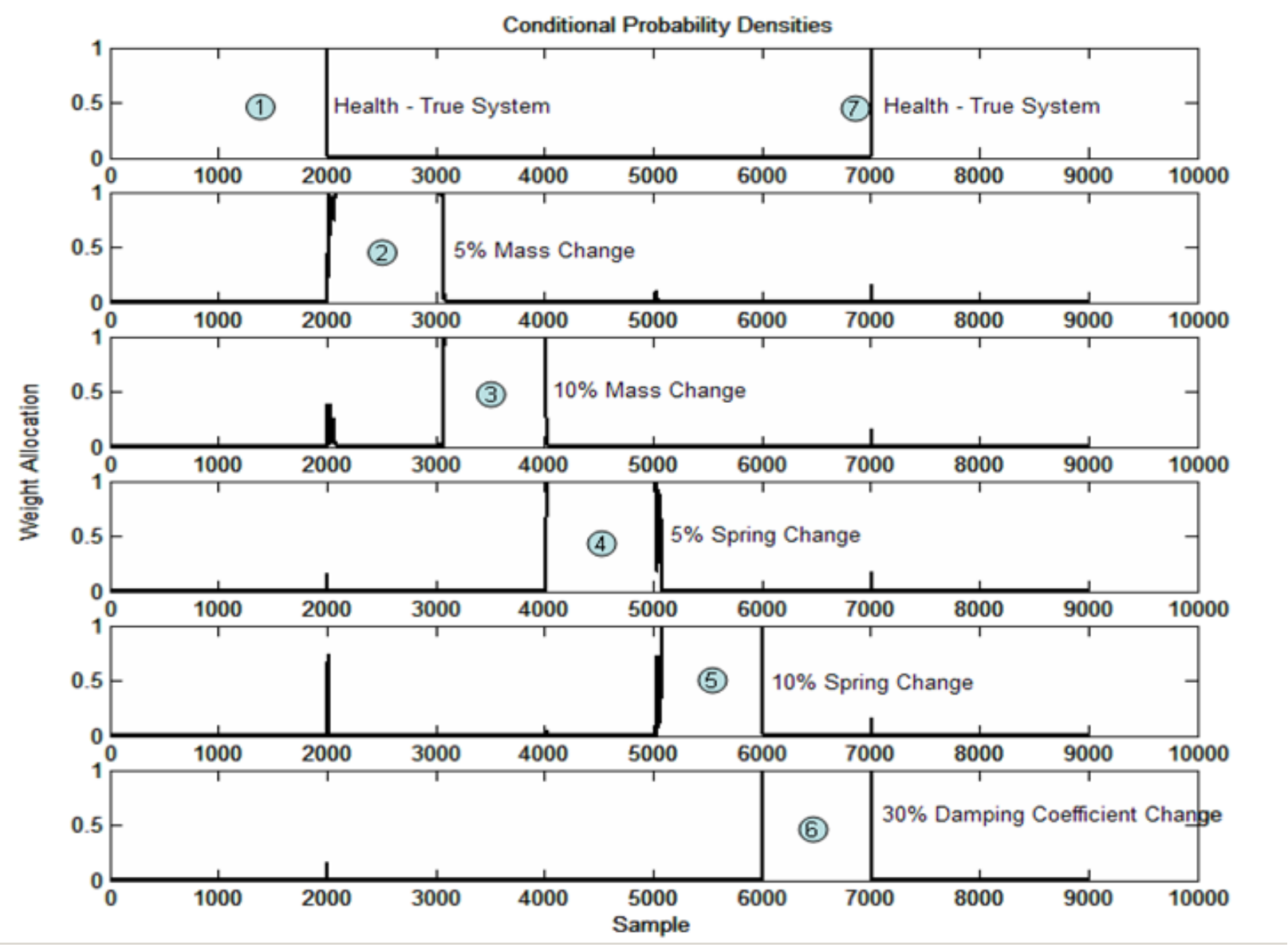

Fig. 5.2.4.1.4 Conditional probability density function outputs over different cases of the fault scenario. Higher output values show the validity of the case and as a result the diagnosis of the fault. Numbers 1 through 7 show different steps of fault scenario. 


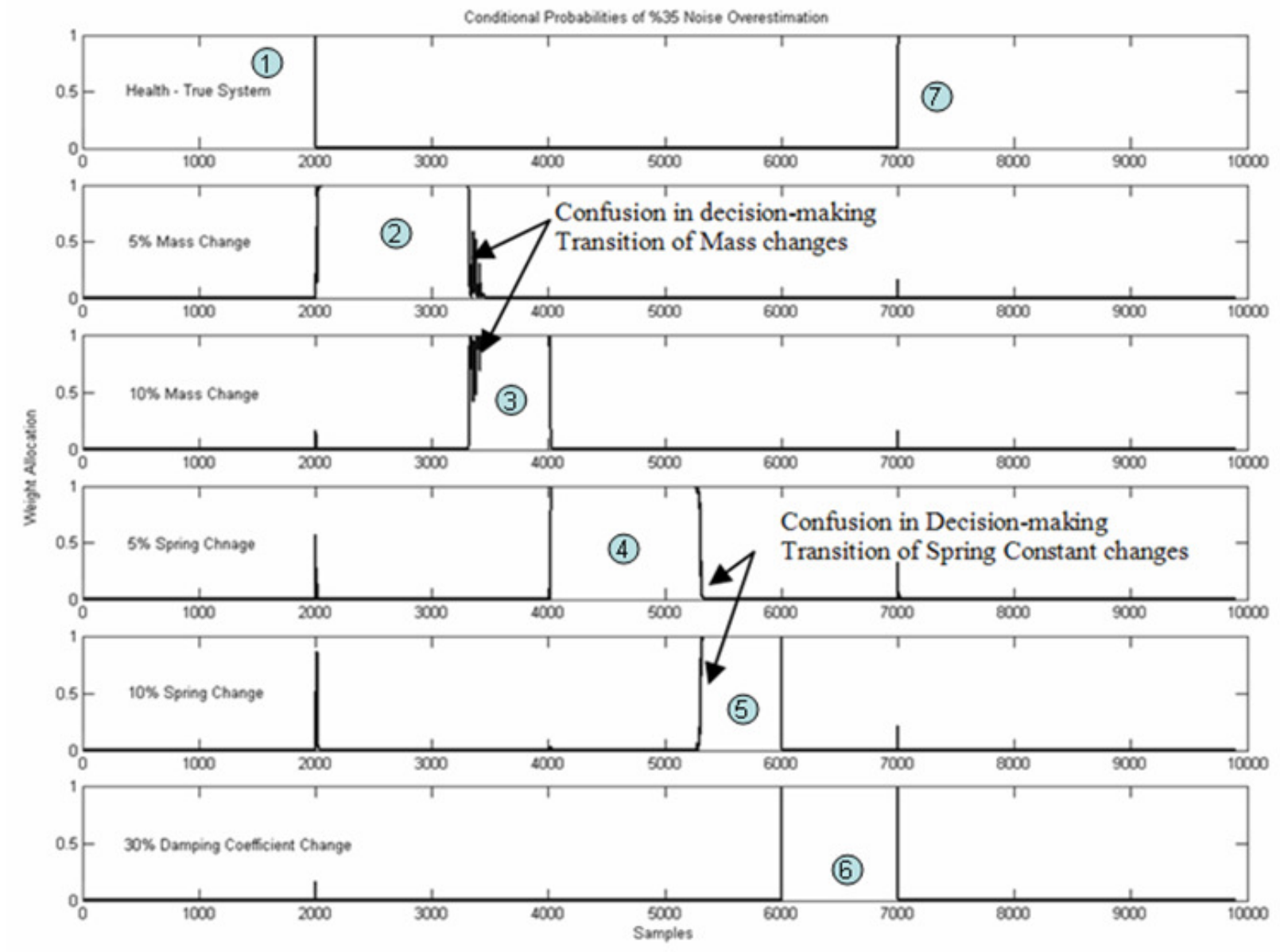

Fig. 5.2.4.1.5. Conditional probability function outputs over different cases of the fault scenario in a $35 \%$ noise overestimation. Higher output values show the validity of the case and as a result the diagnosis of the fault.

If the noise content in the system is underestimated, the results show inaccurate diagnosis and random transitions among fault scenario steps, e.g. in transitions from steps 2 to 3 , steps 3 to 4 and 4 to 5. Figure 5.2.4.1.6 shows the noise underestimation results and its consequences on the decision-making process. 


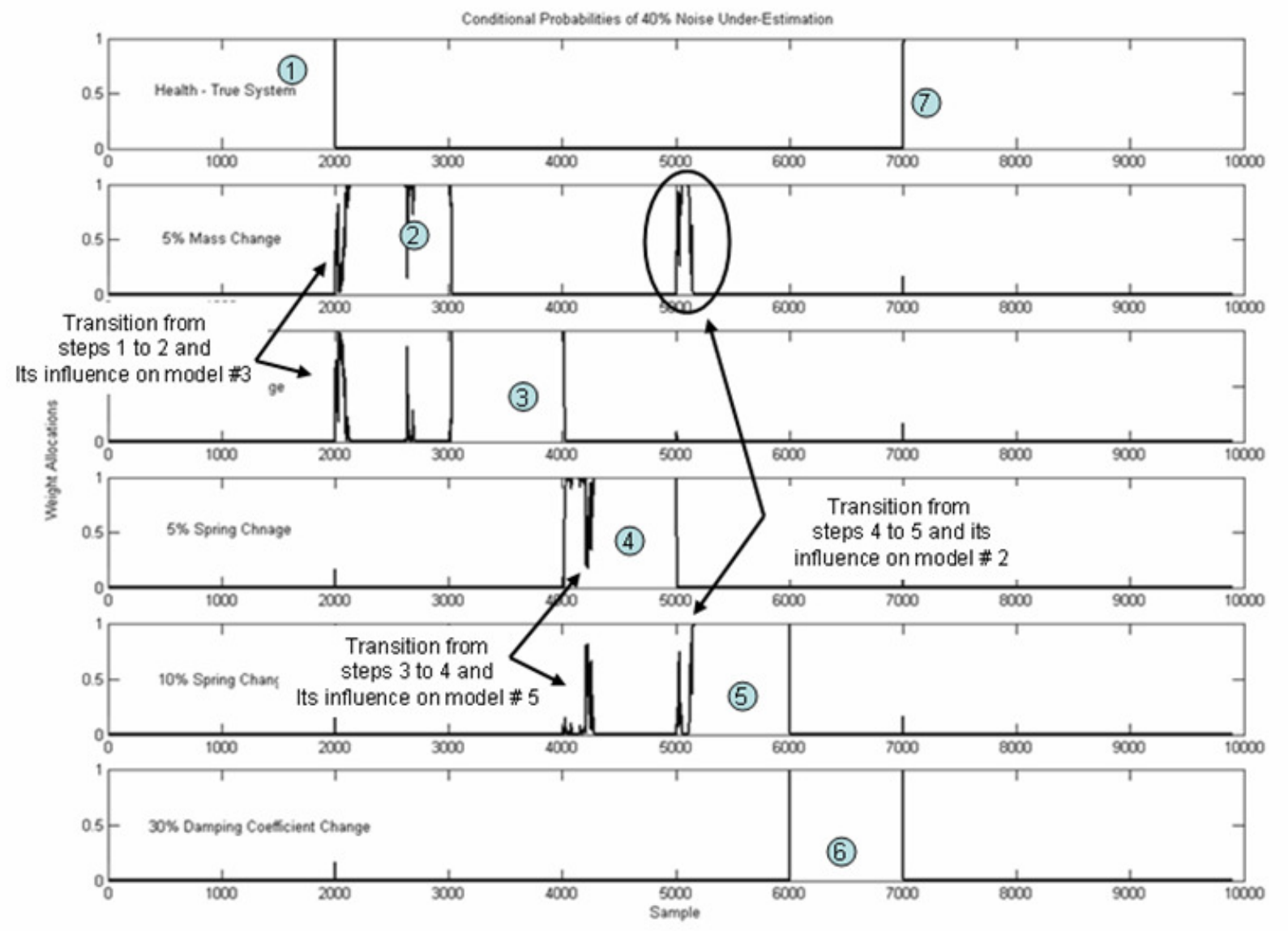

Fig. 5.2.4.1.6. Conditional probability function outputs over different cases of the fault scenario in case of $40 \%$ noise underestimation. Higher output values show the validity of the case and as a result the diagnosis of the fault in that

\subsubsection{Experimental Results and Discussion:}

In order to experimentally examine the fault diagnosis performance in physical implementations, two MEMS comb resonators were designed and fabricated. The parameters of the systems, shown in Table 5.2.4.2.1, contained almost the same amount of mass, but due to imperfect manufacturing steps different damping coefficients and spring constants were obtained. In order to measure the displacement of the device, optical through wafer displacement monitoring technique was utilized.

Devices with different parameters result in various displacements even if excited by the same input. For experiments, the fabricated LCRs were excited by the same input and their output was joined together to form the parameter variation or fault with a transition point at the 1886th sample. The displacement encoded optical data for these devices is shown in Figure 5.2.4.2.1. Recovered displacement signal is shown in Figure 5.2.4.2.2. Interested readers are referred to (Izadian, Hornak \& Famouri. 2006), (Wang, Dawson, Hornak, Famouri \& R. Ghaffarian. 2004), (Dawson, Wang, Chen, Famouri \& Hornak. 2000), (Dawson, Chen, Brown, Famouri \& Hornak. 
2000), (Park, Horowitz \& Tan. 2001), ( Wang. 2005), (Dawson, Wang, Chen, Famouri \& Hornak. 2000), (Wang, Dawson, Chen, Famouri \& Hornak. 2000), for optical displacement monitoring technique.

These devices were excited by a $10(\mathrm{v}), 2 \mathrm{kHz}$ sine wave voltage at sampling time of $h=1.93 e-8 \mathrm{sec}$.

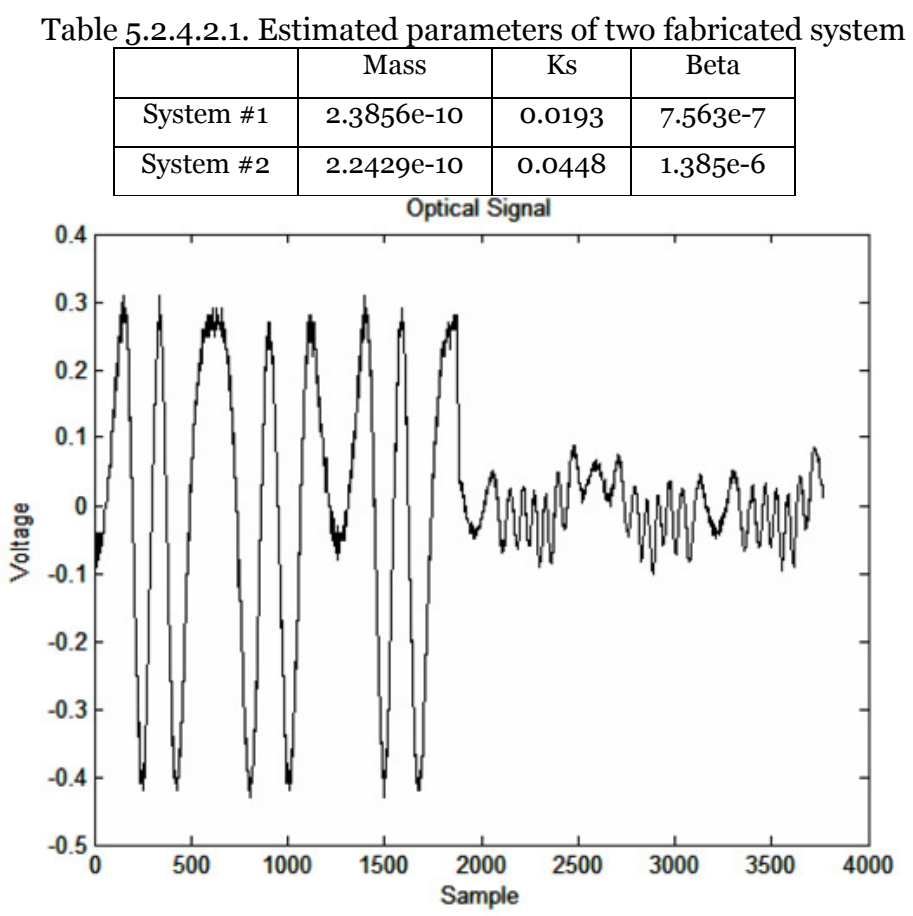

Fig. 5.2.4.2.1. Optical signal, grating encoded displacement signals.

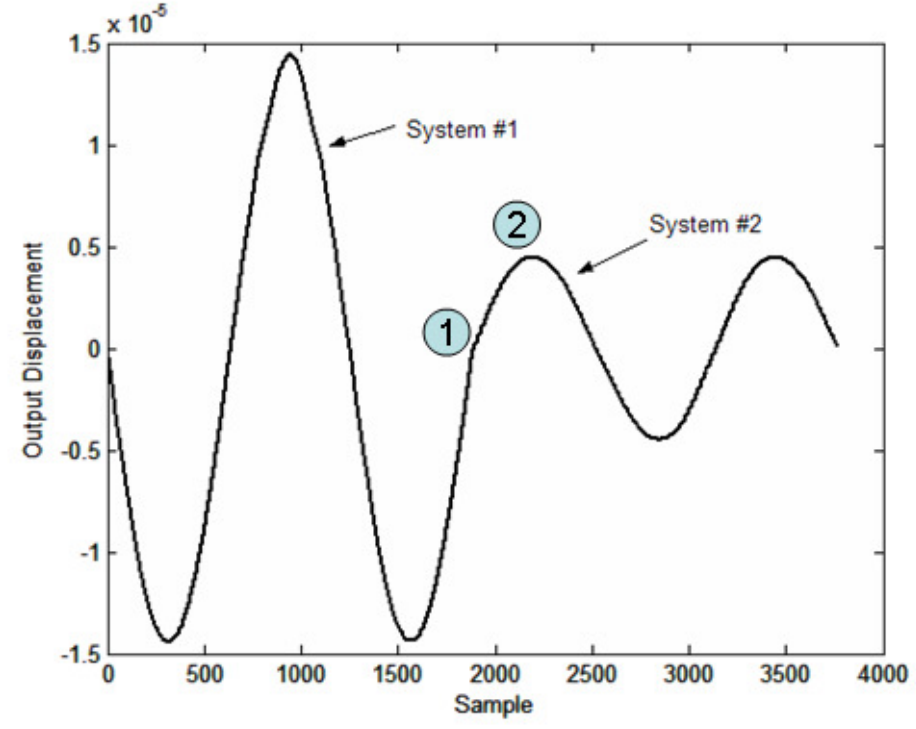

Fig. 5.2.4.2.2. Recovered displacement of LCRs. Point 1 shows the starting of slope change and point 2 shows the switching point from system 1 to 2 . 
Kalman filters were designed for these devices with their gains shown in table 5.2.4.2.2. Initial probability density values of these systems were set equally, and their Kalman gains were computed. Figure 5.2.4.2.3shows the weight allocation and transitions in the fault diagnostic unit. As Figure 5.2.4.2.3 illustrates, higher probabilities are associated to system 1, making it valid for samples up to 1886 also marked as point 1 in Figure 5.2.4.2.3. There is small variation observed in the probability values because of the noise in the system. A quick transition in the probability values is observed when the fault occurs and the weight is shifted to validate the second system (point 2 in Fig 5.2.4.2.3).

Table 5.2.4.2.2. Kalman filtering gains for systems 1 and 2.

\begin{tabular}{|c|c|}
\hline Ke1 & Ke2 \\
\hline 0.0013 & 0.0013 \\
& \\
-1.5064 & -3.5741 \\
\hline
\end{tabular}

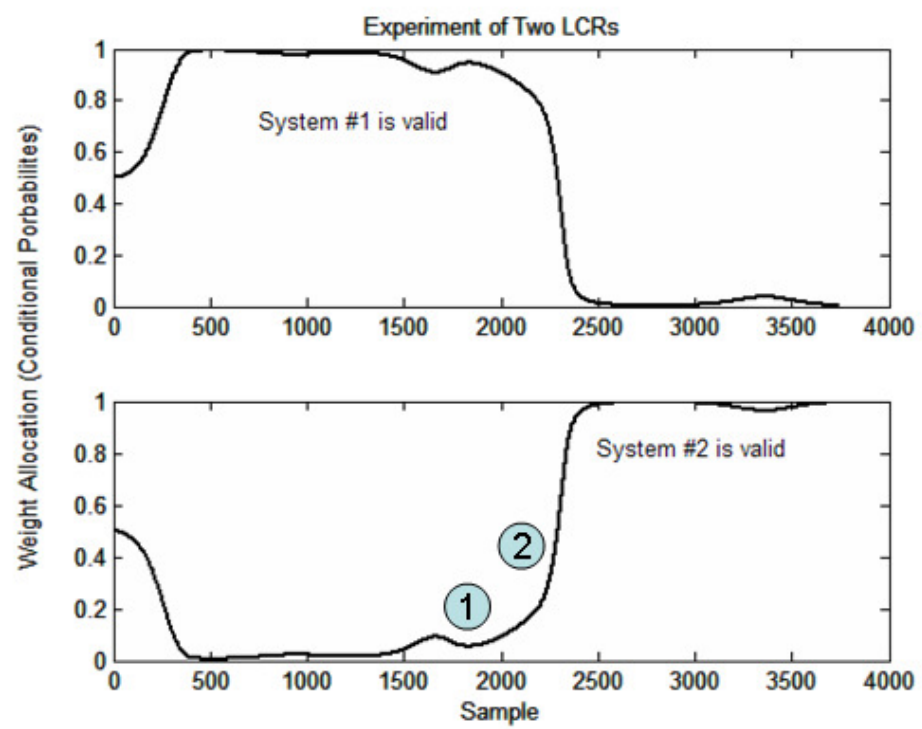

Fig. 5.2.4.2.3. Weigh allocation and conditional probability densities from test of two fabricated LCRs. Switching from system 1 to system 2 is shown. 


\subsection{Self-Tuning Banks and Their Application in MEMS Fault Diagnosis}

\subsubsection{Introduction}

Multiple Model Adaptive Estimators generally reveal the behavior of linear time invariant systems by weighting the output of several models simultaneously according to input-output measurements and evaluating their residual signals. Kalman filters are conventionally used in modeling of time invariant systems under different conditions. The history of output variations (in the actual system) also has a considerable effect on the performance of model-matching core. In uncertain or time-varying parameter systems, techniques other than Kalman filters are required for model-matching and weight allocations. Effect of recent data (variations) has to be controlled to accurately identify the parameters of systems and result in high performance modelmatching unit.

Some of model-matching units introduce local states for local models and accept possible transients (Kuipers \& Astrom 1994). Graphical structures are also applied to describe the transitions among models which require a priori knowledge of the system (Whittaker 1990). Operating systems, however, are modeled by different techniques such as piecewise modeling, spline, and statistical approach, fuzzy and neural networks (Bellman 1961), (Opoitsev,-,), (Stromberg, Gustafsson \& Ljung 1991), (Wahba 1990), (Powell,-—).

In slowly time varying parameter systems, in addition to the model-matching core, there is a need for a unit to identify the model parameters and its variations. The parameter identification unit may be replaced with Kalman filter banks in conventional MMAE configurations. Like Kalman filters, the identification unit has to consider the noise in system and compute the output deviations to generate residual signals. Since the history of variations (output of actual system) affects the performance of model-matching, Forgetting Factor Recursive Least Square (FFRLS) is applied to weight the recent data appropriately and to identify the parameters of the system accurately (Zhuang 1999). Thus, MMAE applied in time varying parameter systems will contain several system identification units and one model matching core.

In this section, the main purpose is to design a system for fault diagnosis of MEMS devices. Since the parameters of MEMS slowly vary over time, forgetting factor technique is used. The system identification unit (in form of self-tuning blocks) and model-matching core are combined for a second order mass-spring-damper system (model of MEMS). FFRLS is usually used for single input single output systems; however, for multi input and multi output systems a proper number of identifiers can estimate the whole system parameters. The algorithm is implemented for fault diagnosis of two fabricated Lateral Comb Resonators in experiments, and for intentionally simulated faults during the operation. The results of the system identification and fault diagnosis are compared with those obtained from Kalman filters without application of system identification unit. 


\subsubsection{System Modeling}

Different contingencies of actual system are modeled in form of separate sub-models. The actual system which can also be influenced by noise and parameter variations is expressed in state space form as

$$
\begin{aligned}
& x\left(t_{i}\right)=\Phi x\left(t_{i-1}\right)+\Gamma u\left(t_{i-1}\right)+G w\left(t_{i}\right), \\
& z\left(t_{i}\right)=H x\left(t_{i}\right)+v\left(t_{i}\right)
\end{aligned},
$$

where $x\left(t_{i}\right)$ is the state space variable at time $t_{i}, \Phi$ is the system matrix with slowly-varying parameters, $\Gamma$ is the input matrix, $u$ is the input vector, $G$ is the noise input matrix and $z$ is the output measurement vector of the model. $H$ is the output matrix, $w\left(t_{i}\right)$ is a zero mean discretetime white noise in the input and $v\left(t_{i}\right)$ is a zero mean discrete-time white measurement noise in the output of the system. The variances of system $(Q)$ and measurement noise $(R)$ are independent values, which might change in each model, and are defined as

$$
\begin{gathered}
E\left\{w\left(t_{i}\right) w^{T}\left(t_{j}\right)\right\}=\left\{\begin{array}{l}
Q, t_{i}=t_{j} \\
0, t_{i} \neq t_{j}
\end{array},,\right. \\
E\left\{v\left(t_{i}\right) v^{T}\left(t_{j}\right)\right\}=\left\{\begin{array}{l}
R, t_{i}=t_{j} \\
0, t_{i} \neq t_{j}
\end{array},\right.
\end{gathered}
$$

Parameters of model $(5 \cdot 3 \cdot 2.1)$ can be adjusted according to different conditions and parameter variation. For instance, an estimation of the $\mathrm{k}^{\text {th }}$ sub-system is expressed as

$$
\begin{aligned}
& \hat{x}_{k}\left(t_{i}\right)=\hat{\Phi}_{k} \hat{x}_{k}\left(t_{i-1}\right)+\hat{\Gamma}_{k} u\left(t_{i-1}\right), \\
& \hat{z}_{k}\left(t_{i}\right)=\hat{H}_{k} \hat{x}_{k}\left(t_{i}\right),
\end{aligned}
$$

where $\left({ }^{\wedge}\right)$ sign indicates the matrices and state variables of estimated values. Sub-models, shown in Figure 5.3.1.1, are connected in parallel with self-tuning banks (contain the system identification algorithm); therefore, each sub-model is represented separately in form of (5.3.2.4). In other words, sub-models are specific representations of the system behavior under different conditions. Parameter identification blocks in form of self-tuning banks can also estimate the output of these sub-systems. Estimated output signals when compared to the output of the actual system generate residual signals and are used to compute the weights applied to the estimated 
state space variables.

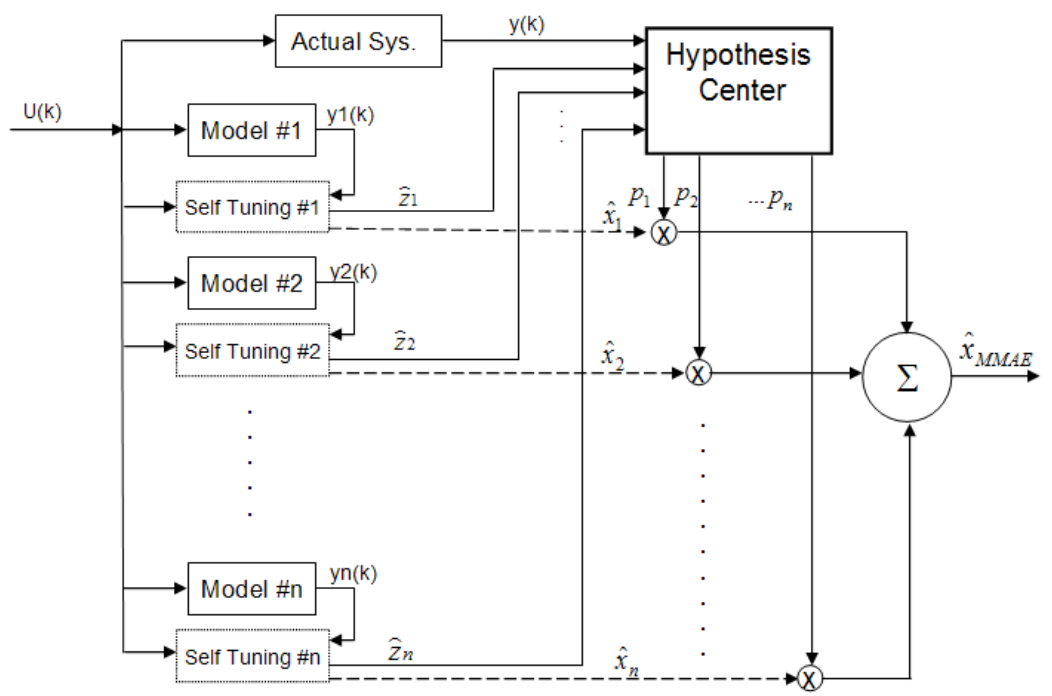

Fig. 5·3.1.1. Multiple-Model Adaptive Estimator using self-tuning banks.

\section{5•3.3 Theory Development}

\section{5•3.3.1 Self-Tuning Estimator}

The main purpose of a self-tuning unit is to estimate the parameters and output of a system. In slowly-varying parameter systems, Recursive Least Square (RLS) technique is applied. A forgetting factor is utilized where the history of variation affects the performance of estimation.

Consider an Auto Regressive Moving Average (ARMA) system representing the $k^{\text {th }}$ sub-system which is expressed as

$$
z_{k}\left(t_{i+1}\right)=b_{0} u\left(t_{i}\right)+\ldots+b_{m} u\left(t_{i-m}\right)-a_{1} z_{k}\left(t_{i}\right)-\ldots-a_{n} z_{k}\left(t_{i-n+1}\right)
$$

where $a_{i}$ 's are the coefficients of the denominator polynomial (input polynomial), and $b_{i}$ 's are the coefficients of the numerator (output polynomial) of the system and $u, z_{k}$ are the input and output signals of the $\mathrm{k}^{\text {th }}$ sub-system. The unknown parameter values of the system are listed in a vector as

$$
\theta^{T}=\left[b_{0} b_{1} \ldots b_{m}, a_{1} a_{2} \ldots a_{n}\right]
$$

The size of unknown vector is $l=m+n+1$ where $m+1, n$ represent the order of the numerator and denominator polynomials respectively. Model's input-output sets can form a regressor matrix as 


$$
h^{T}\left(t_{i}\right)=\left[u\left(t_{i}\right) u\left(t_{i-1}\right) \ldots u\left(t_{i-m}\right),-z_{k}\left(t_{i}\right),-z_{k}\left(t_{i-1}\right), \ldots,-z_{k}\left(t_{i-n+1}\right)\right] .
$$

The ARMA system can then be presented in a compact form as

$$
z_{k}\left(t_{i+1}\right)=h^{T}\left(t_{i}\right) \theta\left(t_{i}\right)
$$

The estimated output is obtained by substituting an estimate of parameter values $\hat{\theta}\left(t_{i}\right)$ in $(5 \cdot 3 \cdot 3 \cdot 4)$ as

$$
\hat{z}_{k}\left(t_{i+1}\right)=h^{T}\left(t_{i}\right) \hat{\theta}\left(t_{i}\right)
$$

Estimated parameters of the system are carried out recursively utilizing the RLS identification algorithm as (Wellstead \& Zarrop 1991)

$$
\hat{\theta}\left(t_{i+1}\right)=\hat{\theta}\left(t_{i}\right)+\eta\left(t_{i}\right) h\left(t_{i}\right)\left\{1+h^{T}\left(t_{i}\right) \eta\left(t_{i}\right) h\left(t_{i}\right)\right\}^{-1}\left\{z\left(t_{i+1}\right)-h^{T}\left(t_{i}\right) \hat{\theta}\left(t_{i}\right)\right\},
$$

where $\eta(t)$ is the covariance matrix which is defined and updated as

$$
\eta\left(t_{i+1}\right)=\eta\left(t_{i}\right)-\left\{1+h^{T}\left(t_{i}\right) \eta\left(t_{i}\right) h\left(t_{i}\right)\right\}^{-1}\left\{\eta\left(t_{i}\right) h\left(t_{i}\right) h^{T}\left(t_{i}\right) \eta\left(t_{i}\right)\right\}
$$

Applying the Forgetting Factor Recursive Least Square (FFRLS), time varying parameters are estimated by introducing forgetting factor $\lambda$ as follows: (Wellstead \& Zarrop 1991)

$$
\hat{\theta}\left(t_{i+1}\right)=\hat{\theta}\left(t_{i}\right)+\eta\left(t_{i}\right) h\left(t_{i}\right)\left\{\lambda+h^{T}\left(t_{i}\right) \eta\left(t_{i}\right) h\left(t_{i}\right)\right\}^{-1}\left\{z\left(t_{i+1}\right)-h^{T}\left(t_{i}\right) \hat{\theta}\left(t_{i}\right)\right\}
$$

The covariance matrix, $\eta(t)$ is also updated considering the forgetting factor as

$$
\eta\left(t_{i+1}\right)=\frac{1}{\lambda}\left[\eta\left(t_{i}\right)-\left\{\lambda+h^{T}\left(t_{i}\right) \eta\left(t_{i}\right) h\left(t_{i}\right)\right\}^{-1}\left\{\eta\left(t_{i}\right) h\left(t_{i}\right) h^{T}\left(t_{i}\right) \eta\left(t_{i}\right)\right\}\right],
$$

where $0<\lambda<1$.

Residual signals which are defined as differences between the actual system's output and those of sub-models, are obtained by 


$$
r_{k}\left(t_{i}\right)=y\left(t_{i}\right)-\hat{H}_{k} \hat{x}_{k}\left(t_{i}\right)
$$

\section{5•3.4 Fault Diagnosis Application in MEMS: Simulation and Experiment}

\subsubsection{Simulation:}

Consider the fault scenario designed in section 5.2.4.1. The FFRLS estimation algorithm was initialized by $P=1 e 8^{*}$ eye(4) with forgetting factor of $\lambda=0.86$. Figure $5 \cdot 3.4 .1$ demonstrates the performance of diagnosis in each step of fault scenario by allocating a probability value to each of the models. Higher probability values (closer to 1) indicate a match of the system to that particular subsystem. For instance, in step 1 (from the scenario), the system operated under normal conditions, and simulations confirm this fact by allocating a high probability to the subsystem number 1.

The speed of transitions from one-step to another and probability variations during validity of a model indicate the performance of diagnosis. As Figure 5.3.4.1 shows, there is no significant delay observed in transitions, and the probability is smooth in validity of a model.

Initializing the system identification at different forgetting factors obtains different diagnosis profiles. At higher forgetting factors, distortions were observed in transitions among models. As it is illustrated in Figure 5.3.4.2, at $\lambda=0.9$ the diagnosis profile shows distortion in transition from step 2 to step 3 of fault scenario ( +5 and $+10 \%$ mass change cases). The self-tuning banks could estimate the parameters considering the existence of noise in the system, and the fault diagnosis algorithm could identify the rest of variations properly during a short time. There was no significant delay observed in transitions among other steps of fault scenario. 


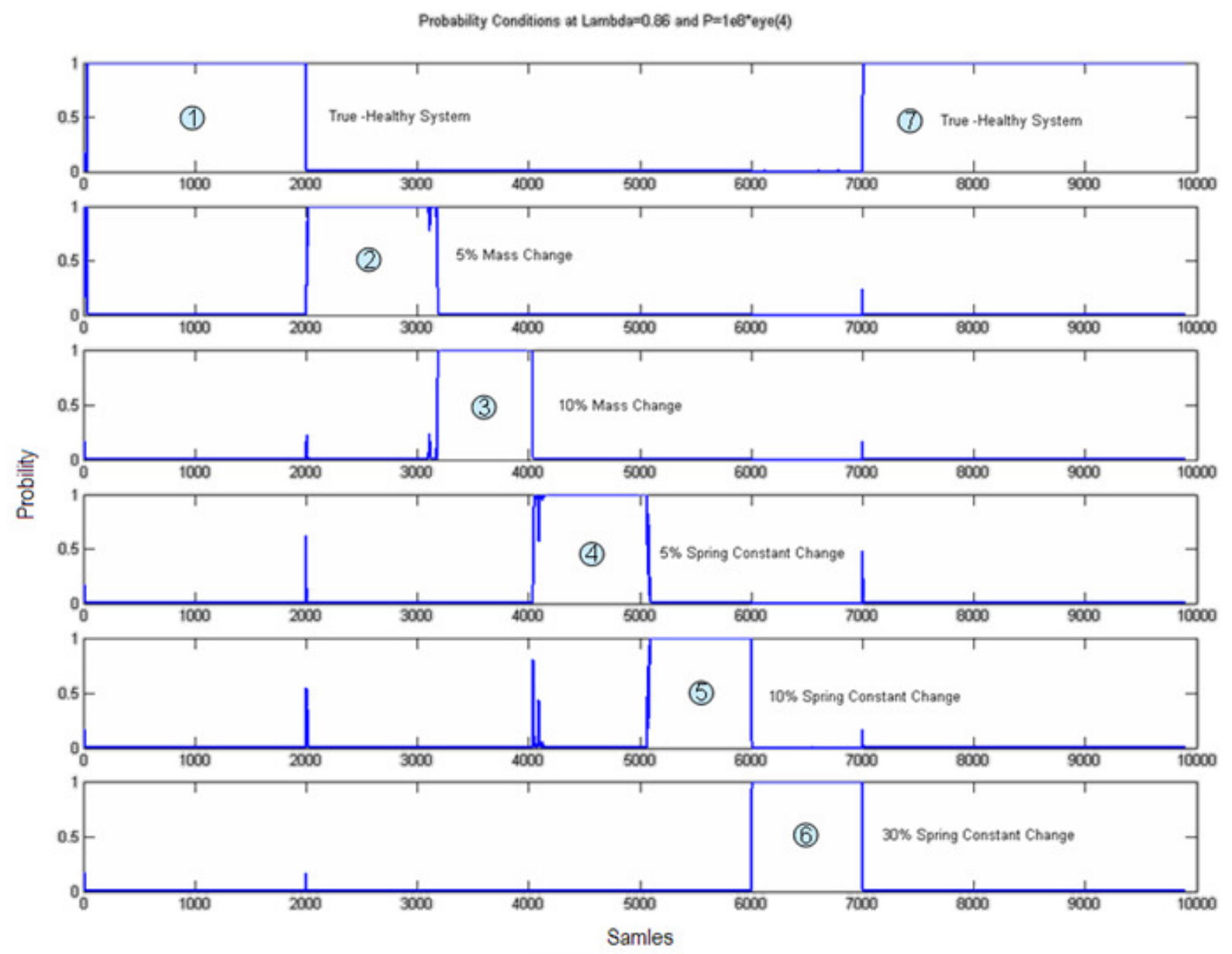

Fig. 5.3.4.1. Conditional Probability values according to the fault scenario with FFRLS at $\lambda=0.86, P=1 e 8 * I$. 


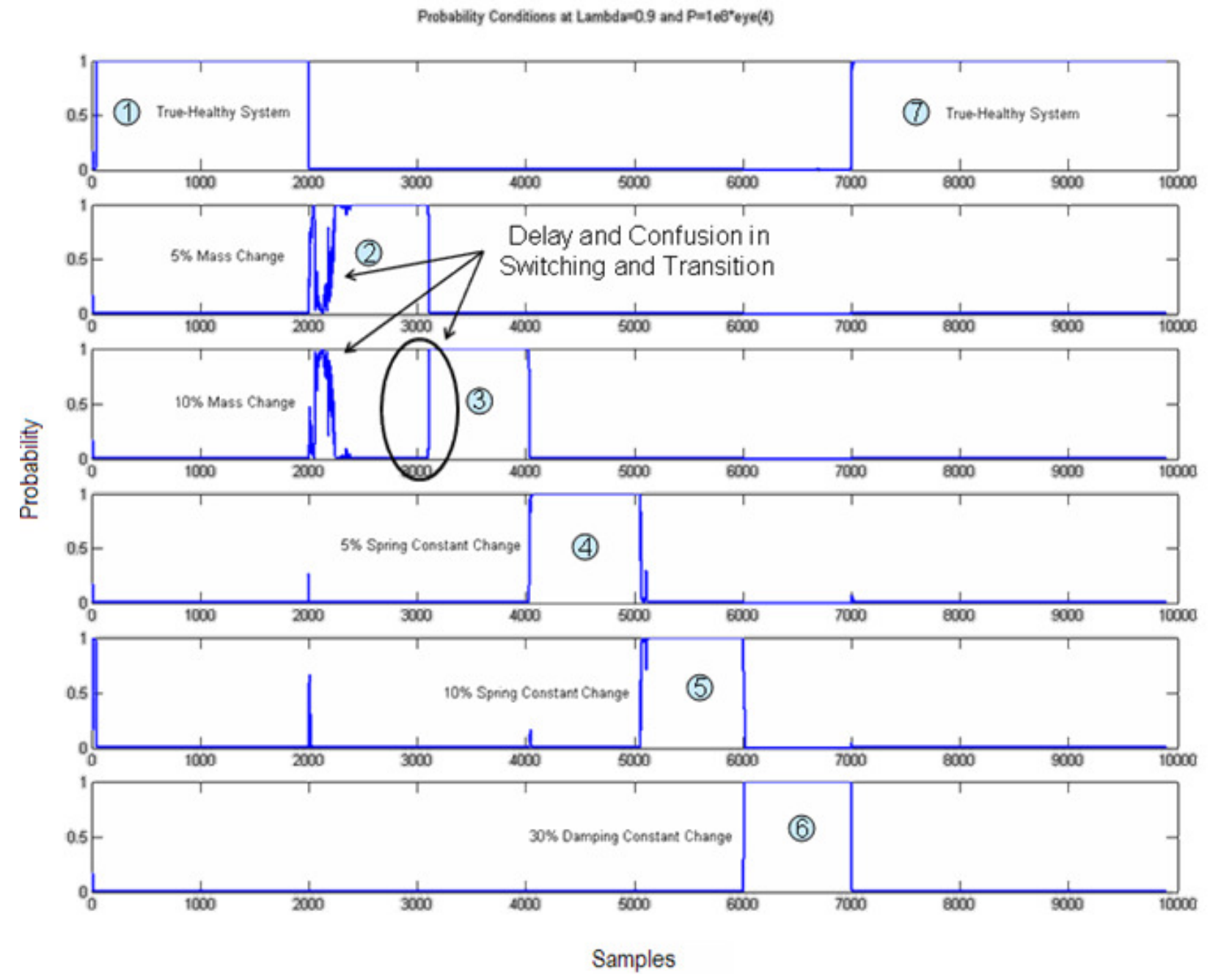

Fig. 5.3.4.2. Conditional Probability values according to the fault scenario with FFRLS at $\lambda=0.9, P=1 e 8 * I$.

Increasing the forgetting factor to higher values, resulted in a short history of data for the conditional probability density functions and initiated delays in weight computations.

As shown in Figure 5.3.4.3, the fault diagnosis was completely dysfunctional at $\lambda=0.98$, wherein wrong diagnosis was observed in steps 1 and 4, and steps 6 to 7 . 


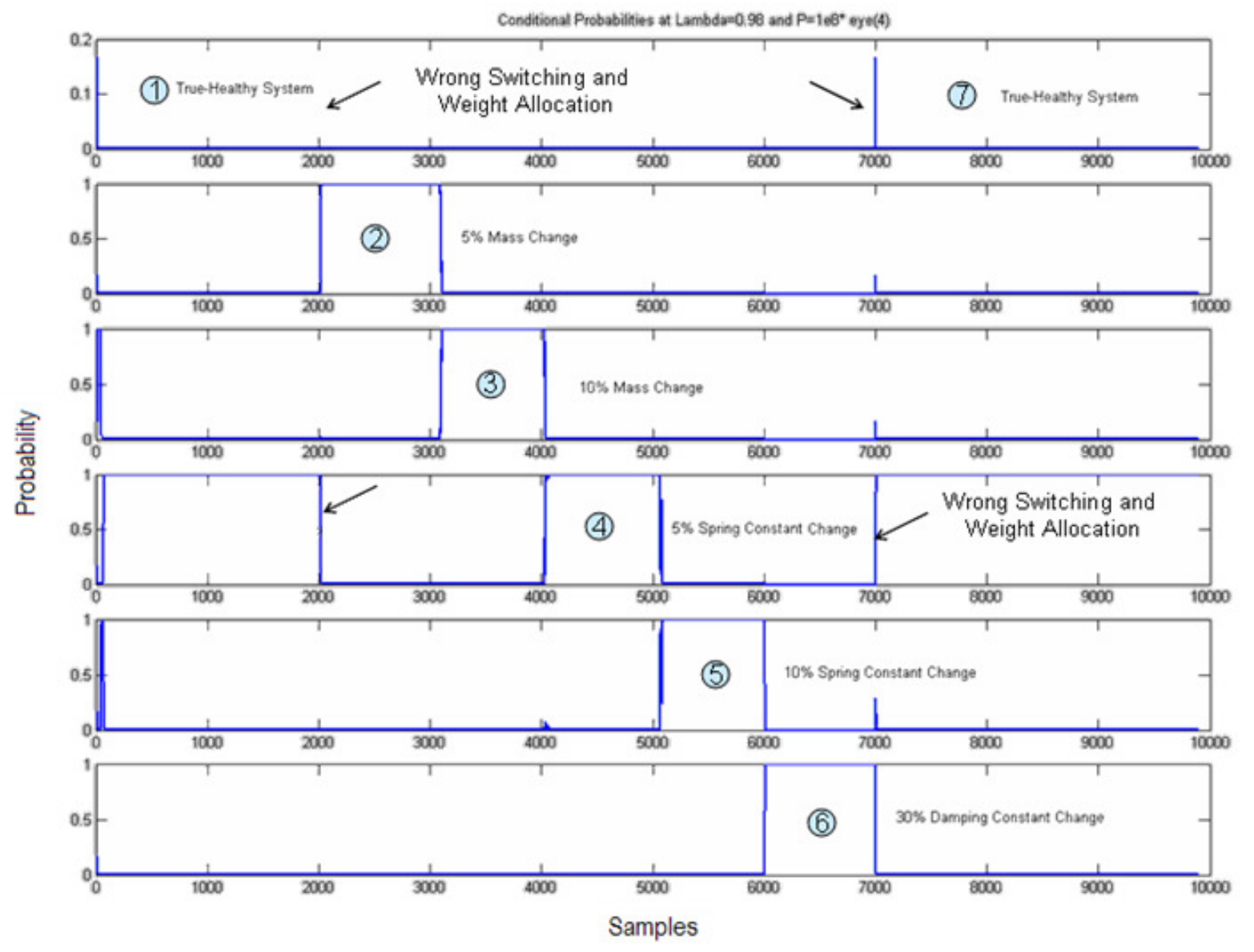

Fig. 5.3.4.3. Conditional Probability values according to the fault scenario with FFRLS at $\lambda=0.98, P=1 e 8 * I$.

According to the level of noise in the system and the rate of parameter variations, satisfactory transitions and precise parameter estimation were observed in the range of $0.86<\lambda<0.9$. Values out of this boundary change the history of data and cause distortions in transitions. Self-tuning banks could successfully estimate the sub-system parameters and output signals at a suitable forgetting factor value and result in a robust decision making unit under different conditions.

\section{5•3.4.2 Experimental Results:}

In order to examine the capabilities of the application of self-tuning estimator banks in combination with MMAE technique to diagnose the type of fault in MEMS devices, two Lateral Comb Resonators were designed and fabricated. In the design of these devices, spring constants were intentionally considered different but after fabrication, due to the imperfect manufacturing steps, there was also a variation observed in the damping coefficient of the device. The parameters of the fabricated devices are shown in table 5.2.4.2.1, which reveal almost the same 
mass content, a $130 \%$ variation in spring constant and $83 \%$ variation in damping coefficient. LCR devices were excited by the same sine wave, separately, and the resultants output waveforms were attached together resembling the fault occurrence at the $1886^{\text {th }}$ sample point. The recorded optical signals (containing the displacement information) are shown in Figure 5.2.4.2.1, while the devices were excited by a $10 e^{j 2 \pi 2000 t}(\mathrm{v})$ signal. Decoding the grating encoded optical data showed the displacement variation (Fig. 5.3.4.2.1). For more information on optical data decoding please see (Izadian, Wang, Dawson, Hornak \& Famouri 2007), (Wang, Dawson, Hornak, Famouri \& Ghaffarian 2004), (Dawson, Wang, Chen, Famouri \& Hornak 2000), (Dawson, Chen, Brown, Famouri \& Hornak 2000), (Dawson, Brown, Famouri, \& Hornak 2000), (Dawson, Wang, Chen, Famouri \& Hornak 2000), (Wang, Dawson, Chen, Famouri \& Hornak 2000).

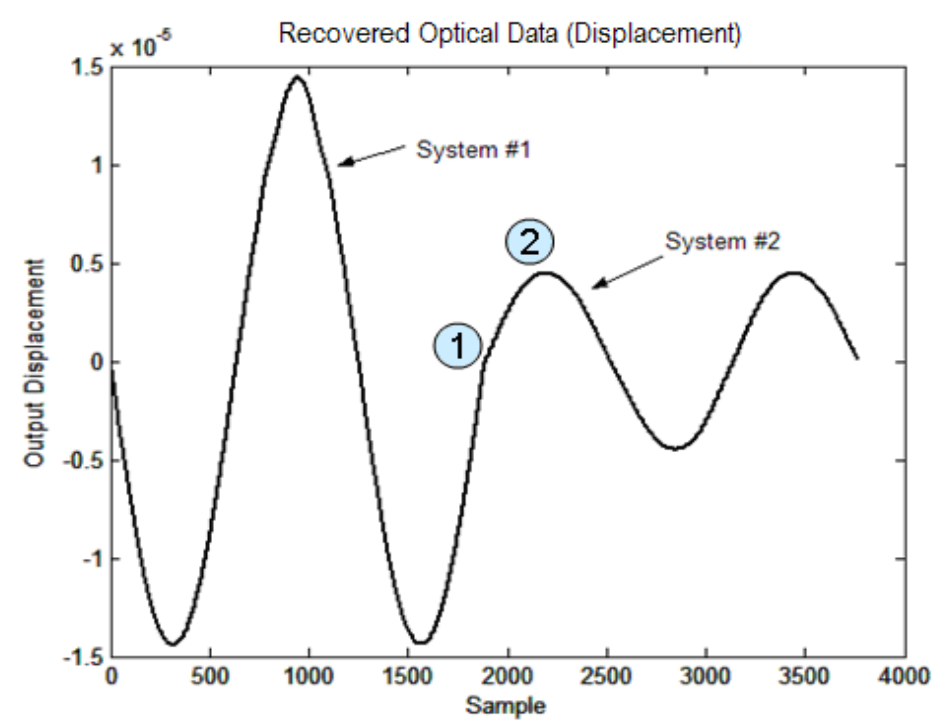

Fig. 5.3.4.2.1. Recovered displacement of LCRs. Point 1 shows the starting of slope change and point 2 shows the switching point from system 1 to 2 . 

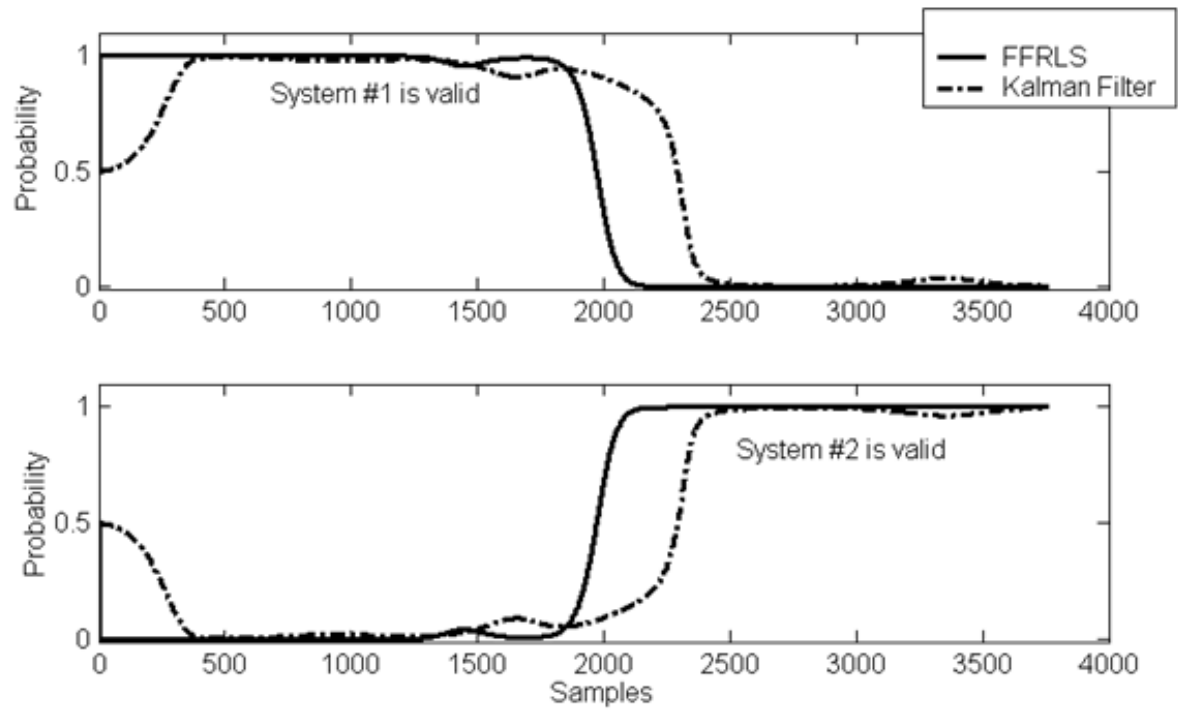

Fig. 5.3.4.2.2. Weight allocation and conditional probability densities from test of two fabricated LCRs. Switching form system 1 to system 2 is shown at $\lambda=0.8$.

Self-tuning banks generated two sets of state space representations for each system at $\lambda=0.8$ shown in table 5.3.4.2.1. They contained the same input matrices with similar diagonal elements in their system matrices. The effect of parameter variations are more observed in the off-diagonal values of the system matrix.

Applying the MMAE technique and self-tuning estimators together result in the weight allocations and probability computations as shown in Figure 5•3.4.2.2. As the figure shows, highest weight was allocated to system 1 in early steps of the first part, meaning that the actual system behaved similar to sub-system 1 . At the fault point occurrence (sample $1886^{\text {th }}$ ) the weight started shifting toward system 2 and the transition was completed in a short time. There was no significant variation observed in the calculated weights before the fault occurrence moment in the first part. After the fault, system 2 had higher weight and probability values, demonstrating the operation under fault in sub-system 2. The MMAE and self-tuning together could identify the multiple parameter variations resulted from a fault in the system. This demonstrated the successful application of the proposed technique on the MEMS fault diagnosis.

Table 5·3 4.2.1 Self-tuning system identification results for LCR system 1 and 2 using forgetting factor $\lambda=0.8$

\begin{tabular}{|l|l|}
\hline System 1 & $\hat{x}(k+1)=\left[\begin{array}{cc}1.9934 & -0.9935 \\
1.0000 & 0\end{array}\right] \hat{x}(k)+\left[\begin{array}{c}0.3052 \mathrm{e}-4 \\
0\end{array}\right] u(k)$ \\
\hline System 2 & $\hat{x}(k+1)=\left[\begin{array}{cc}1.9882 & -0.4944 \\
2.0000 & 0\end{array}\right] \hat{x}(k)+\left[\begin{array}{c}0.3052 \mathrm{e}-4 \\
0\end{array}\right] u(k)$ \\
\hline
\end{tabular}




\subsection{Adaptive Forgetting Factor}

In previous section, the application of a fixed forgetting factor was introduced. The fact is that the forgetting factor value was determined based on a time-consuming trail and error procedure which is not always emerging to a specific number for all the systems. By changing the level of noise in the system, a new value of forgetting factor might be required. In this section, the fixed value can be replaced by an adaptive mechanism of optimal adjustment of coefficients according to the history of variations. The optimal value is considered as $\lambda_{i}$ and derived according to the following procedure.

\subsubsection{Online implementation of Adaptive Approach for Optimal Forgetting Factor:}

Let consider the level of noise in the system as a white noise with zero mean and variance as

$$
E\left\{w\left(t_{i}\right) w^{T}\left(t_{i}\right)\right\}=\sigma_{\omega}^{2} R_{i},
$$

where

$$
R_{i}=1+H_{i} P_{i-1}^{\prime} H_{i}^{T}
$$

is the normalized variance with respect to $\sigma_{\omega}^{2}$. In practice and in actual implementations, an unbiased estimation of $R_{i}$ is obtained from the measurement data as

$$
\hat{R}_{i}=\frac{1}{\sigma_{\omega}^{2}} \frac{1}{i-1} \sum_{k=1}^{i} w_{k} w_{k}^{*}, \quad i=1,2, \ldots
$$

This estimation can be calculated recursively by

$$
\hat{R}_{i}=\frac{1}{\sigma_{\omega}^{2}} \frac{\lambda_{i-1} \hat{R}_{i-1}+w_{k} w_{k}^{*}}{\gamma_{i}},
$$

where

$$
\gamma_{i}=1+\lambda_{i-1} \gamma_{i-1}
$$


The initial conditions might be at $\lambda_{0}=1, \hat{R}_{0}=0$ and $\gamma_{0}=0$. The value of $\lambda_{i}$ due to the noise in the system might be larger than 1 . There is almost no model ensuring that the forgetting factor value under all the conditions remains limited within $\lambda_{i} \in(0,1]$ (Zhuang. 1999). Therefore, in practical implementation, there is a necessity of considering a reasonable limit on $\lambda_{i}$. Initially, the value of $\lambda_{i}$ is computed as

$$
\lambda_{i}=\frac{\lambda_{i-1}\left(1+H_{i} P_{i-1}^{\prime} H_{i}^{T}\right)}{\hat{R}_{i}},
$$

where $P_{i}^{\prime}=\frac{P_{i}}{\lambda_{i}}$. As it was mentioned earlier, for limiting the forgetting factor to $\lambda_{i} \in(0,1]$, the following procedure should be considered,

$$
\lambda_{i}=\lambda_{i-1}+\mu \cdot \operatorname{sgn}\left(\lambda_{i}-\lambda_{i-1}\right)
$$

where $s g n$ is the signum function and $\mu$ is the step size determined according to the parameter variation rate and noise. For online implementation of adaptive forgetting factor, there is no need of estimating $\sigma_{\omega}^{2}$. In computer simulations according to the SNR range of targeted application the value can be set.

\subsubsection{Experiment:}

The same devices as those of used in the previous sections were used and for the forgetting factor an adaptive approach was applied. The initial forgetting factor and variance were considered as $\lambda_{0}=1, \hat{R}_{0}=0$ and the step size was considered as $\mu=0.0001$.

Figure 5.4.2.1 shows the weight allocation for the system. A fast transition from system \#1 to system \#2 shows the fault diagnostic ability of the adaptive forgetting factor algorithm. As it is seen, the exact value of forgetting factor is not required, and its initial value started at one. 

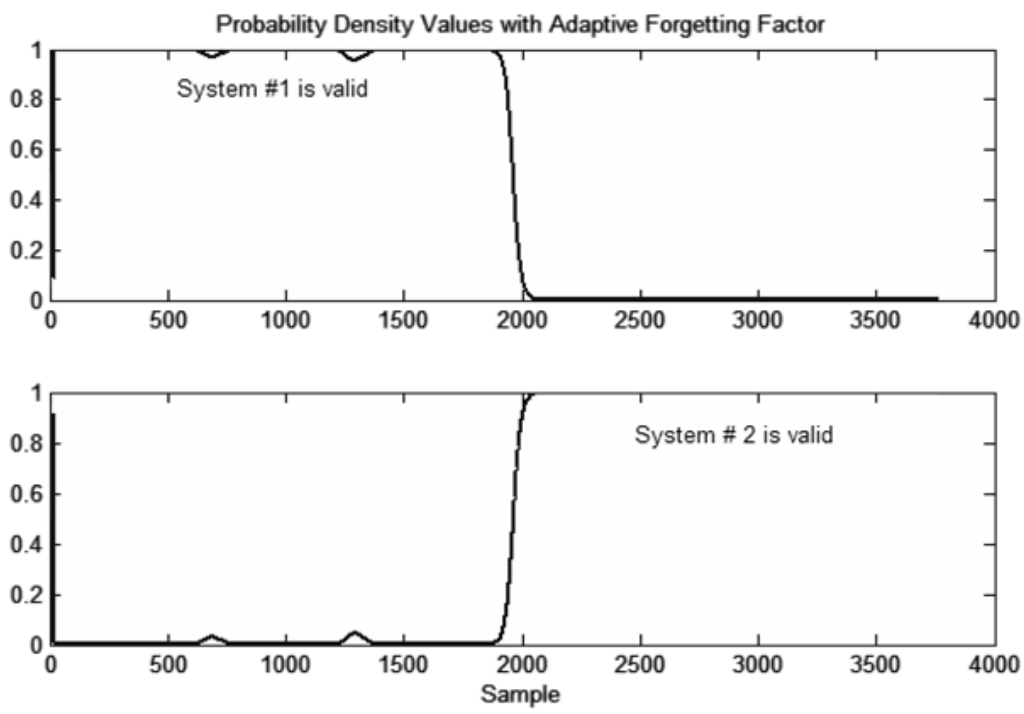

Figure 5.4.2.1. Probability Density Values of Self Tuning with Adaptive Forgetting Factor
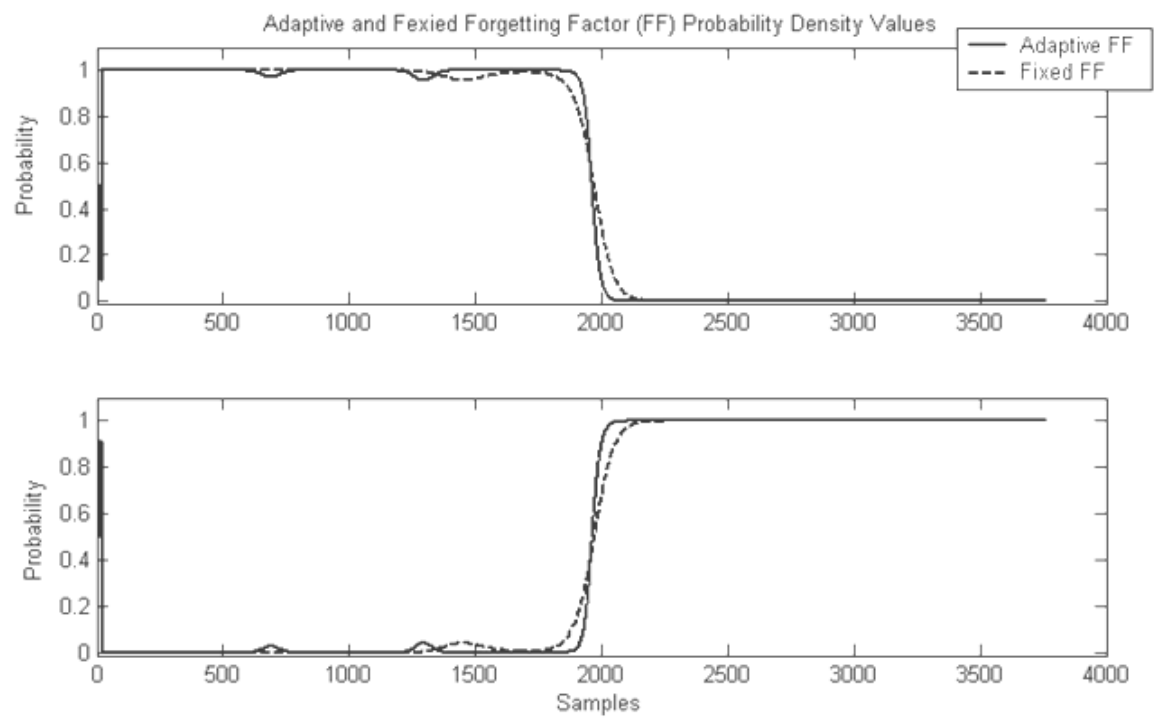

Figure 5.4.2.2. Comparison of the Probability density values of the fixed and Adaptive Forgetting Factor.

Comparing the fixed and adaptive forgetting factors show quicker decision making in adaptive forgetting factor case although the fault is sensed earlier in the fixed case, the transition was faster in the adaptive forgetting factor case (Figure 5.4.2.2). 

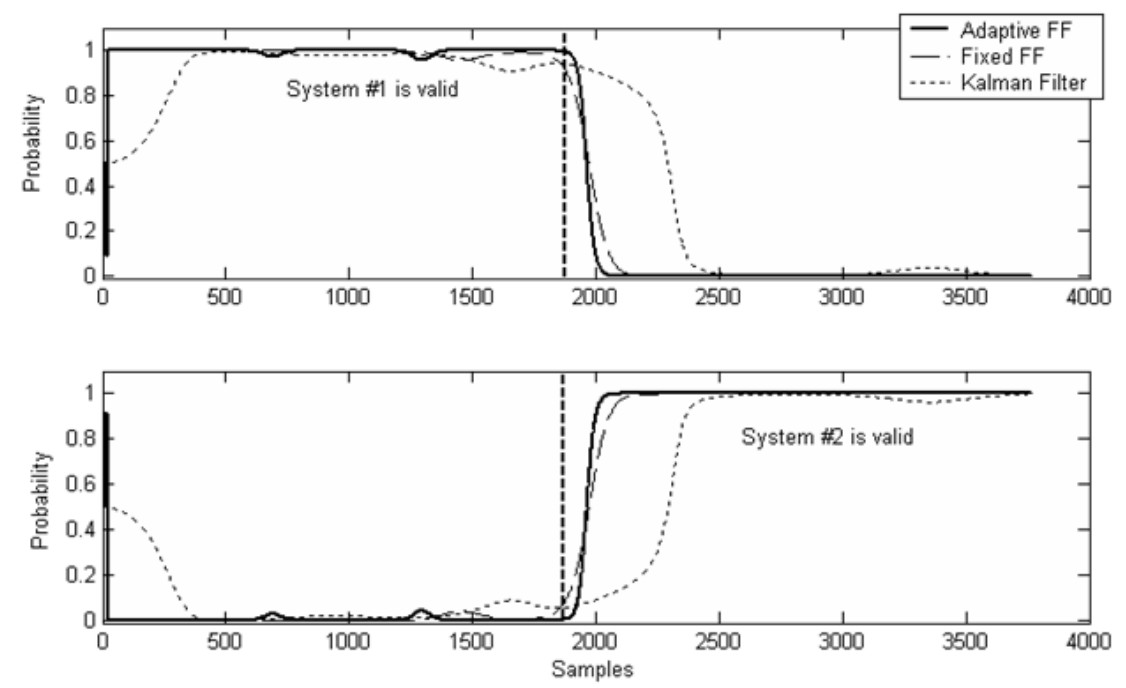

Figure 5.4.2.3 experimental results and comparison of the Kalman filter and Self-Tuning with Fixed forgetting Factor and Adaptive Forgetting Factor

As the Figure (5.4.2.3) shows, the Kalman filter generates a high delay in diagnosis of the fault. Fixed forgetting factor shows faster system and quicker transitions in decision-making and fault diagnosis. Adaptive forgetting factor shows the best results among three techniques by demonstrating of a sharp fault diagnosis system.

\subsection{Hardware Limitations}

As mentioned earlier, the Kalman filter implementation in MMAE enabled the fault diagnosis technique in MEMS applications. The main limitation in this regard is the hardware power. A good measure of this power can ensure enough number of models and length of each of fault contingencies. Number of FLOPS is measured in each iteration to give a good sense of hardware requirement in transitions and system expectations. Moreover, adding a model to the system will make the system's behavior closer and increases the FLOPS requirements.

It is seen that each iteration requires almost 4335 extra FLOPS, which is almost the same as adding a model to the set. Following table shows the FLOPS incremental rate in iterations and models. 
Table 5.5.1. Flops Analysis in Model and Iteration Increments

\begin{tabular}{|c|c|c|c|c|c|}
\hline \multirow{2}{*}{ Iteration } & \multirow{2}{*}{ Model Increase } & \multicolumn{3}{|c|}{ FLOPS } & \multirow{2}{*}{ Comments } \\
\hline & & TOTAL & \multicolumn{2}{|c|}{ Increment } & \\
\hline 1 & 1 & 2471485 & \multirow{2}{*}{4313} & \multirow{3}{*}{4339} & \multirow{4}{*}{ FLOPS/Iterations } \\
\hline 2 & 1 & 2475798 & & & \\
\hline 3 & 1 & 2480137 & \multirow{2}{*}{4339} & & \\
\hline 4 & 1 & 2484472 & & & \\
\hline 2000 & 1 & 11141412 & \multirow{2}{*}{4335} & & \multirow{2}{*}{ FLOPS/MODEL/Iteration } \\
\hline 2001 & 2 & 11145755 & & & \\
\hline 3000 & 2 & 15478414 & \multirow{2}{*}{4343} & & \multirow{2}{*}{ FLOPS/MODEL/Iteration } \\
\hline 3001 & 3 & 15482749 & & & \\
\hline 4000 & 3 & 14985418 & \multirow{2}{*}{4333} & & \multirow{2}{*}{ FLOPS/MODEL/Iteration } \\
\hline 4001 & 4 & 19819751 & & & \\
\hline
\end{tabular}

As conclusion, in more powerful hardware sets higher number of FLOPS can be handled which increases the possibility of higher iterations and/or higher number of models.

\subsection{Closing Remarks}

We have seen that the fault in microsystems might be originated from different sources, which result in different behavior from microsystem. Changes of mass, spring constant and damping coefficient are commonly recognized as main results of the fault. Applying Multiple-Model Adaptive Estimation and Kalman filters provided an effective way of identifying the type of fault occurrence in microsystems. Simulation of a fault scenario showed the feasibility of application of fault diagnosis in MEMS Lateral Comb Resonators. Kalman filters were designed for every faultrepresentative system and were used to generate residual signals to be applied in weight allocation of conditional probability density functions. Application of MMAE on fault diagnosis of LCR was experimentally tested by fabrication of two devices with some similar and some different parameters resembling the fault occurrence in microsystem. This technique can be used in parallel with CARAMEL system-evaluator to generate suitable models for each fault representative model in microsystem and provide an online supervisory fault diagnosis unit.

This chapter also illustrated the application of self-tuning banks to Multiple Model Adaptive Estimator technique. In the case of time-varying or unknown parameter systems, Kalman filters were successfully replaced by the self-tuning banks. Forgetting Factor Recursive Least Square provided enough history of data on parameter variation and led the system to a quick transition among valid contingencies as a performance-control parameter. The proposed method was enabled for MEMS fault diagnosis applications and was experimentally validated on two fabricated LCRs with different parameter values.

Fixed forgetting Factor values showed better performance than Kalman filters, but required a 
trail and error effort to find out the suitable forgetting value for each system. This value might be different under different conditions and each time it required a new adjustment. Adaptive Forgetting Factor adjustment technique showed less effort to find out a suitable varying value for the system. The highest performance and quickest transition and as a result a new and more effective fault diagnosis technique was obtained when the MMAE technique was equipped with self-tuning estimators and made use of Adaptive Forgetting Factor adjustment. 


\section{Chapter 6}

\section{Future Work}

The controllers and techniques developed in this research can be used for other applications to enhance the controllability and performance of the control routines. In addition, the optical technique (Doppler laser) used for lateral displacement can be developed to measure more precise displacements. In this chapter, some of the potential applications of the control technique in addition to optical measurement technique are investigated.

\subsection{Control of Gyroscopes:}

\subsubsection{Resonating Gyroscope}

Most gyroscopes are essential parts of many systems and they are built in large scales, which make them large and expensive. Recent improvements in micro-machining and silicon industry have made possible the design and manufacturing of the precise devices such as gyroscopes in micro scale. These gyroscopes are manufactured several times smaller than the conventional gyroscopes, fabricated in large quantities to reduce the price and increase their general usage. These devices are used in applications such as videogames (Wii), ride stabilization, cars safety, navigation systems, satellites and many more.

\subsubsection{Problems with MEMS Gyroscope}

Generally, manufacturing steps leave the parameters of the system uncertain and provide asymmetry in the structure of the device. This results in misalignment of the actuation mechanism and systematic perturbations in the electrostatic and mechanical forces. Geometrical imperfections and electrical couplings cause degradation of the performance. The performance of a gyroscope is evaluated by some factors such as:

- Resolution

The resolution is measured by the level of the Brownian and sensing circuit noises and is evaluated by the standard deviation of angular rate input. 
- Drift

Drift is the slow variation of the governing functions of a gyro in a long or short term procedure.

- Scale Factor

Scale factor is the change in the output angular rate estimate per unit change of input angular rate.

- Zero-Rate-Output (ZRO)

ZRO is the false output signal which is generated in the absence of rotation.

\subsubsection{Imperfections in MEMS Gyro Manufacturing}

Various manufacturing steps result in deviations from the desired values in parameters of MEMS. The main effect is the rotation of the MEMS device pattern respect to the required reference as shown in Figure 6.1.3.1.

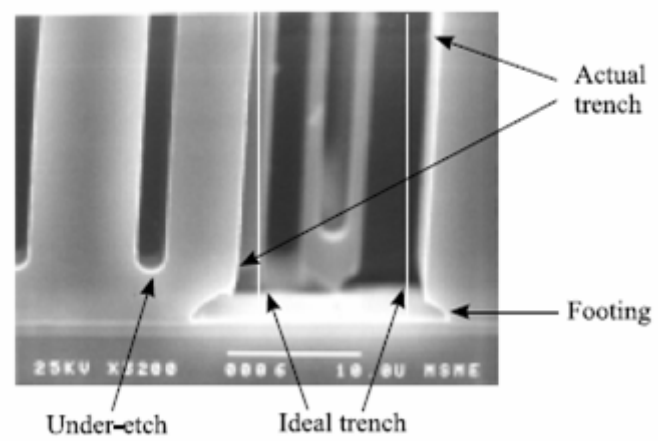

Figure 6.1.3.1: Pattern rotation and the effects on elasticity and spring constant coupling (Park, Horowitz. 2003)

Considering the drive and sense spring constant and damping coefficients, as $k_{1}, k_{2}, d_{1}, d_{2}$ respectively, and pattern rotation angle $\alpha$, the spring constant misalignment as shown in Figure 6.1.3.2, the coupling spring constant are calculated as following (Park, Horowitz. 2003)

$$
\begin{aligned}
& k_{x x}=k_{1} \cos ^{2} \alpha+k_{2} \sin ^{2} \alpha \\
& k_{y x}=\left(k_{1}-k_{2}\right) \sin \alpha \cos \alpha \\
& k_{y y}=k_{1} \sin ^{2} \alpha+k_{2} \cos ^{2} \alpha
\end{aligned}
$$


The same phenomenon occurs for the damping factors. Since they are caused by asymmetry in the aerodynamic drag, structural damping and Coriolis force offset which affect the symmetry of damping terms. Generally, damping axes rotation and those related to elasticity are different since they are originated from different sources. Therefore, the coupling in damping coefficients with respect to their rotation angle are written as (Park, Horowitz. 2003)

$$
\begin{aligned}
& d_{x x}=d_{1} \cos ^{2} \gamma+d_{2} \sin ^{2} \gamma \\
& d_{y x}=\left(d_{1}-d_{2}\right) \sin \gamma \cos \gamma \\
& d_{y y}=d_{1} \sin ^{2} \gamma+d_{2} \cos ^{2} \gamma
\end{aligned}
$$

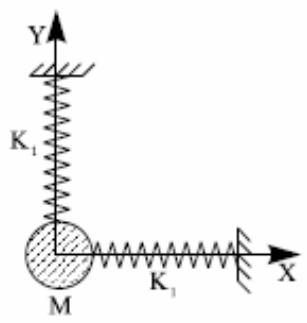

Ideal gyroscope

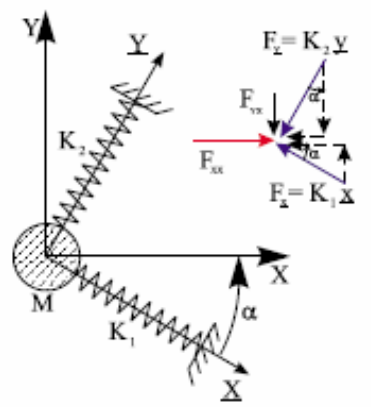

Anisoelasticity in gyroscope

Figure 6.1.3.2, the pattern rotation projections on the $\mathrm{x}$ and $\mathrm{y}$ - axes (Park, Horowitz. 2004)

\subsubsection{Sense Axis Control}

Since fingers on the sense axis combs are designed for measurement of the rotation, synchronizing of outputs of gyros requires manufacturing of some extra fingers to apply coupling force and compensate imperfect manufacturing steps. The difference between the sense and drive axis control configurations are shown in Figure 6.1.4.1. 


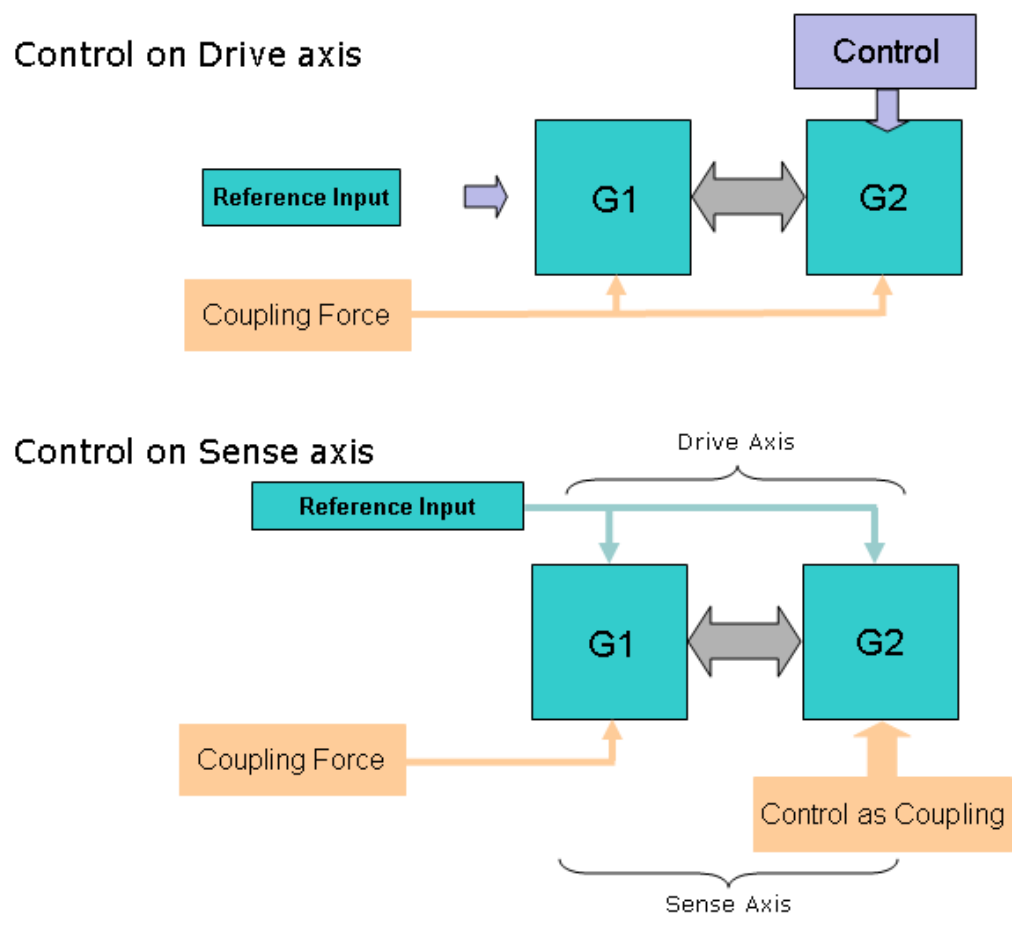

Figure 6.1.4.1, comparison of drive and sense axis controller configurations.

As the picture shows, in drive-axis synchronization, the control command is directly applied to the drive axis fingers, but in sense axis, the control command is applied as coupling force to balance the output of gyros. This requires extra finger manufacturing on the sense combs. Figure 6.1.4.1 shows the same drive signal for two gyros and different coupling force for sense axis to have output of the devices synchronized. The control command in this case is very small comparing to the coupling force and that of the drive-axis. The output of gyros without control on sense axis is shown in Figure 6.1.4.2. After applying controller the performance of the control is shown in Figure 6.1.4.3.

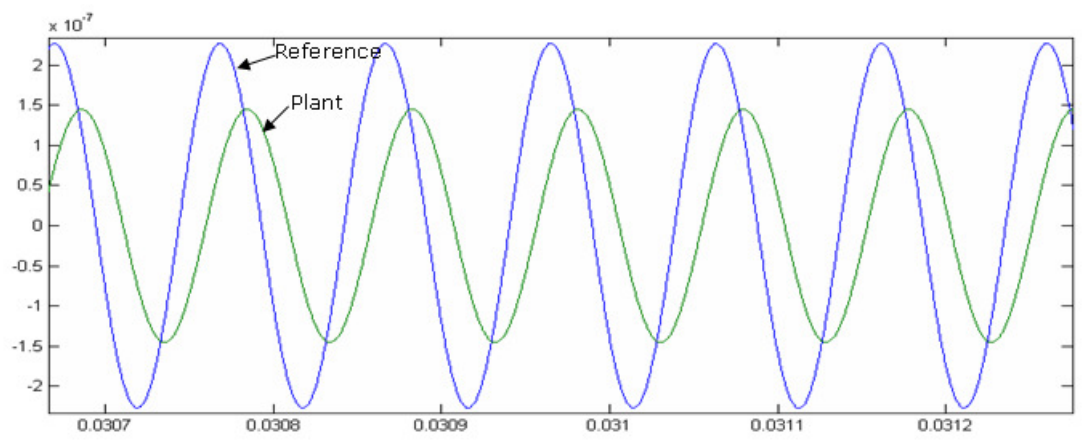

Figure 6.1.4.2, The sense-axis output of two non-identical gyros 


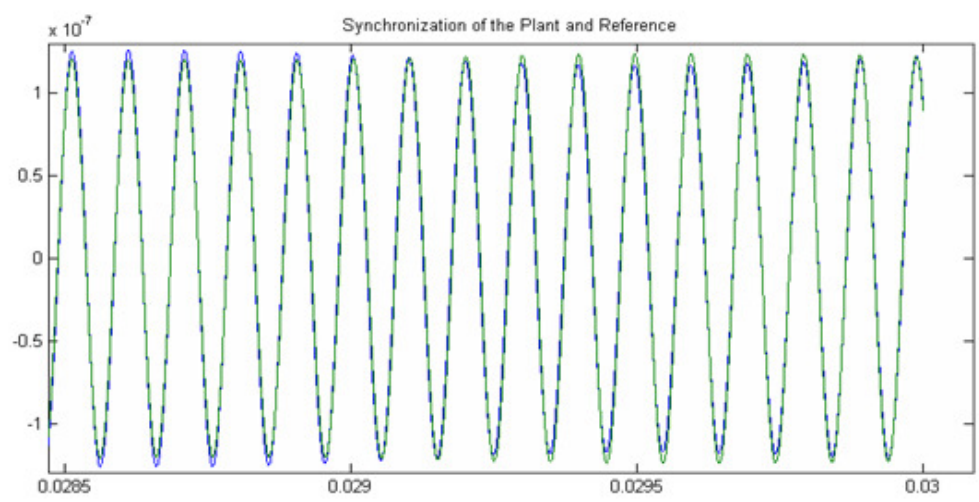

Figure 6.1.4.3, Applying control command on sense axis and synchronization of the measured signals

The control effort in this case, as it was mentioned earlier, is very small. The control effort of the controller is shown in Figure 6.1.4.4 and its coupling force in Figure 6.1.4.5.

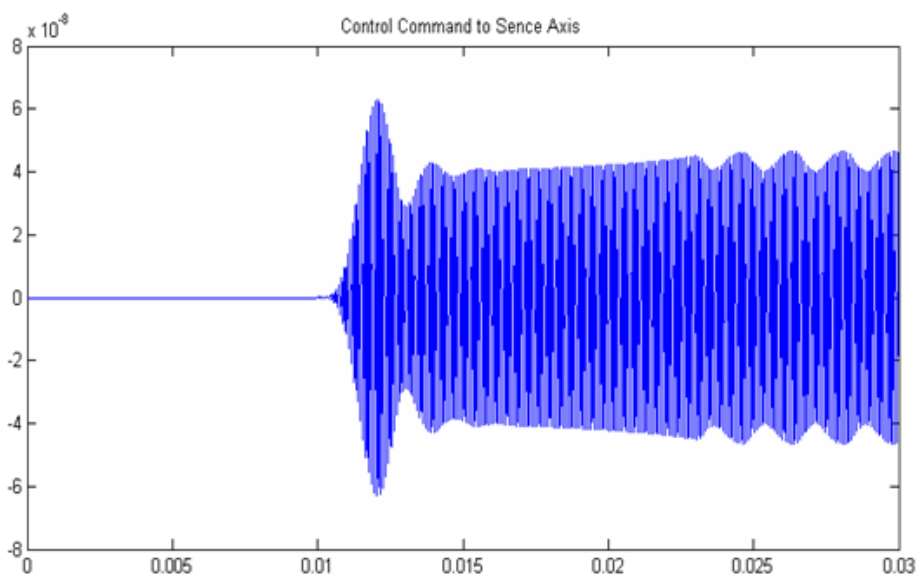

Figure 6.1.4.4, Control command applied on sense-axis 


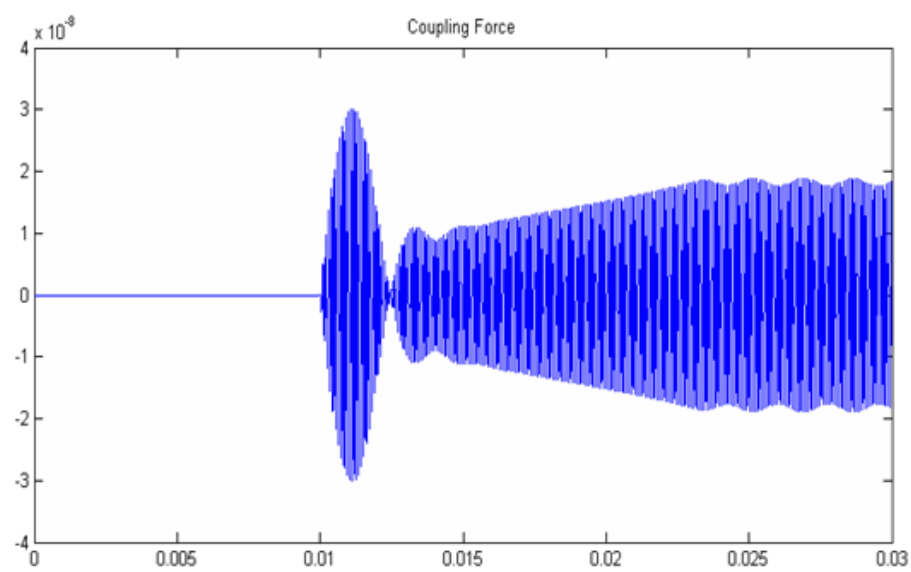

Figure 6.1.4.5, Coupling force between gyros.

As it is seen, the coupling force and the control command to the sense axis are almost in the same range meaning that the applied force to synchronize two devices does not require too many fingers to be manufactured.

\subsubsection{Drift Reduction}

Drift reduction schematic demonstration is shown by monitoring the output of the devices while no rotation is applied to the plane of the gyros. In this case, the control command is applied on the sense axis of the device to synchronize the measurements of the devices. Figure 6.1.5.1 shows the controller configuration in this case.

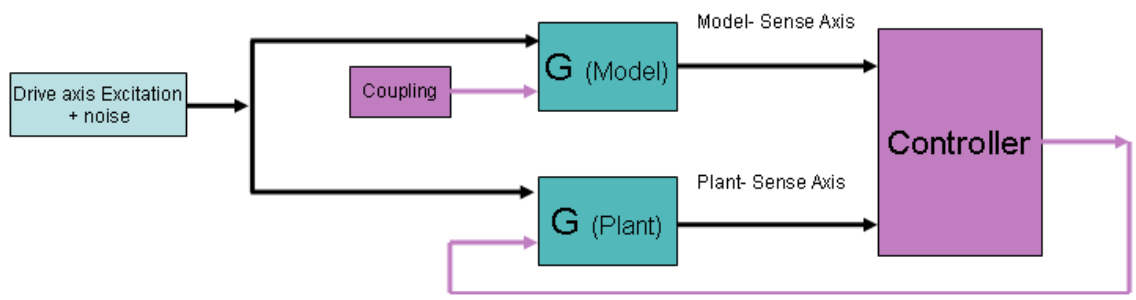

Figure 6.1.5.1, Controller configuration for drift reduction

In the presence of noise, the pattern rotation of the model and the plant gyros contain different values of imperfection. Model is a gyro with anisoelasticity and anisoinertia angles equal to 2 degrees whereas the plant with 4 and 5 degrees respectively. The desired output measurement and the actual measurements are shown in Figure 6.1.5.2. 


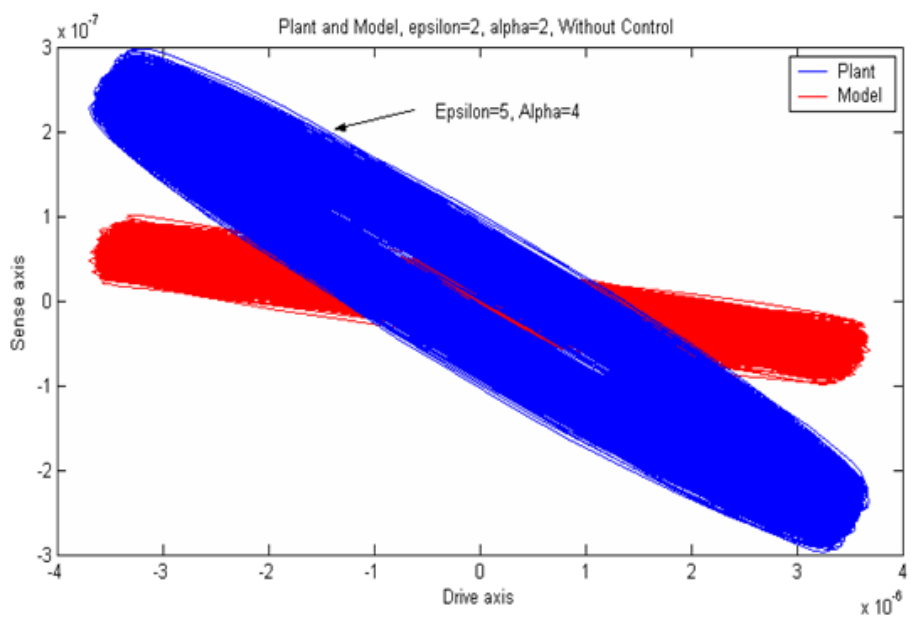

Figure 6.1.5.2, The output locus of two gyros without control command

Applying control command on the sense axis, the synchronized output measurements of two devices are shown in Figure 6.1.5.3.

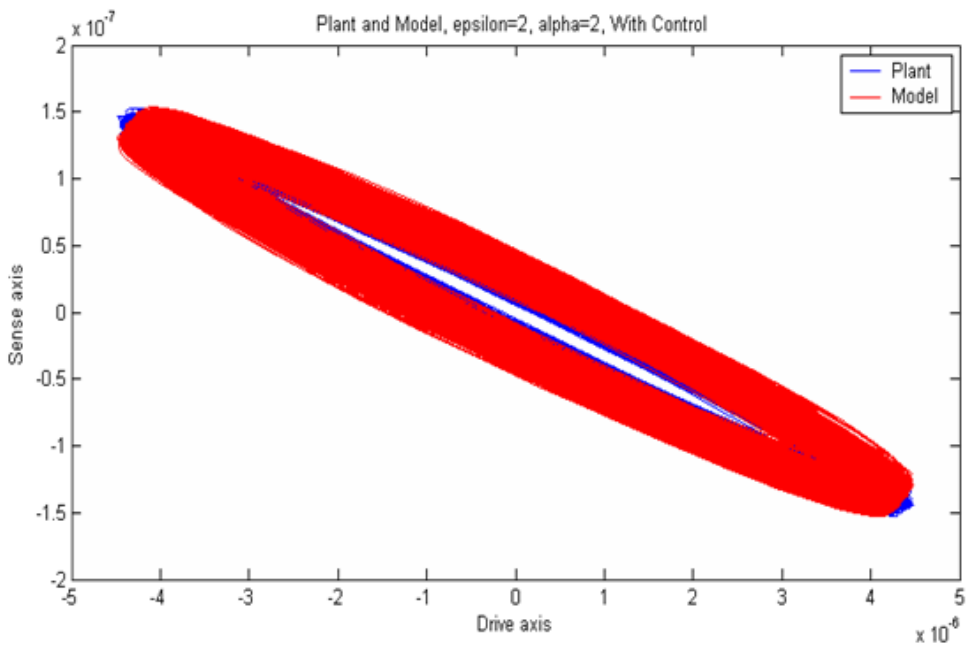

Figure 6.1.5.3, Synchronizations of gyros and reduction of drift problem

Synchronization on the phase of the device is shown in Figure 6.1.5.4. 


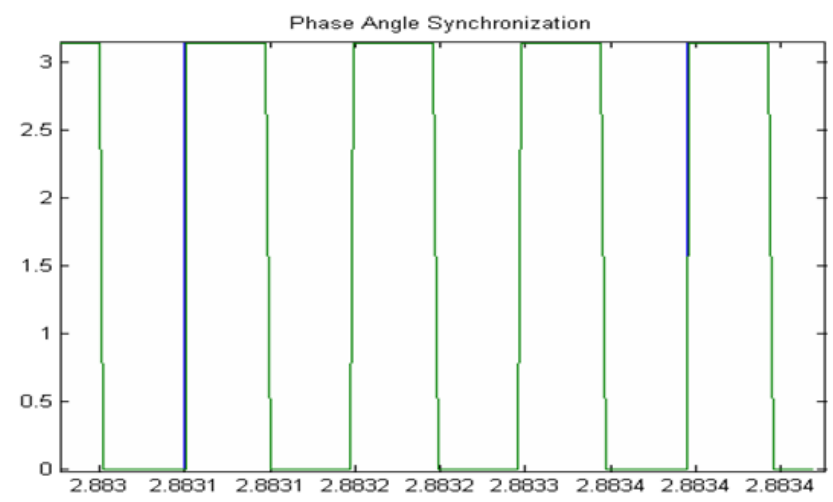

Figure 6.1.5.4. Phase synchronization toward drift reduction.

\subsection{Advances in Control Design of a MIMO System}

The controller designed in this research was used for Single Input Single Output systems. A Multiple Input Multiple Output expansion is more practical since most of the actual system have more than one input and result in more that single output.

\subsection{Multi Beam Optical Monitoring Technique}

In previous chapters, the possibility of using Doppler laser in the openings was demonstrated. The obtained resolution of monitoring was 2 micron. Applying multiple beams or locating the reference beam on the moving stage results in better resolution for the system. The new configuration requires a new data recovery and displacement measurement system. Figure 6.3.1.1 shows a possible location for a two beam system.

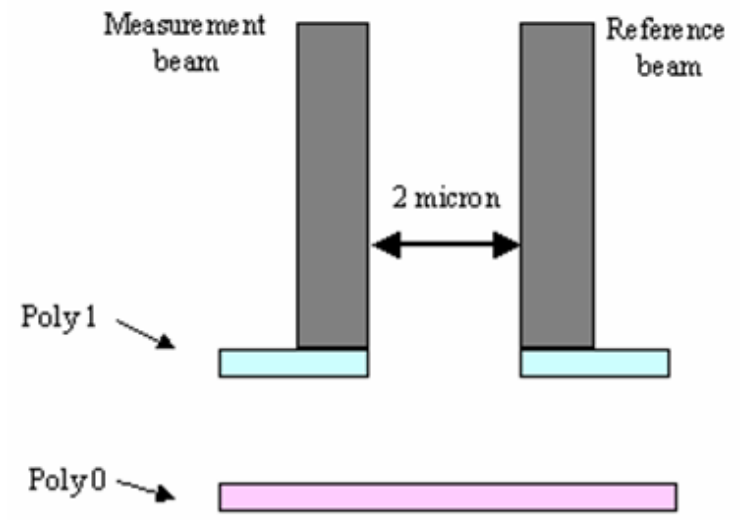

a) Both beams at the same level.

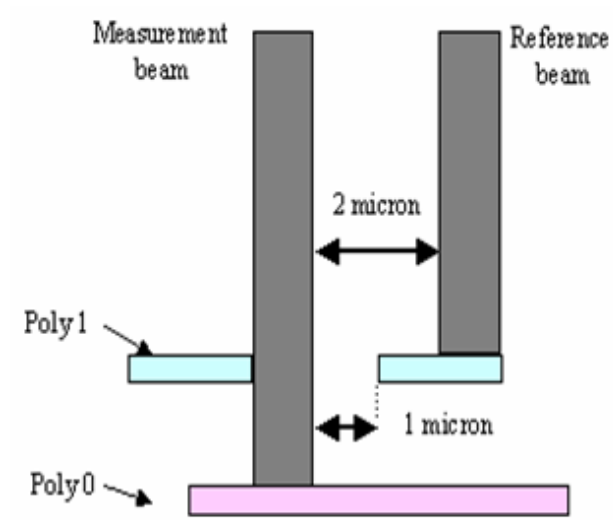

b) 1-micron displacement 


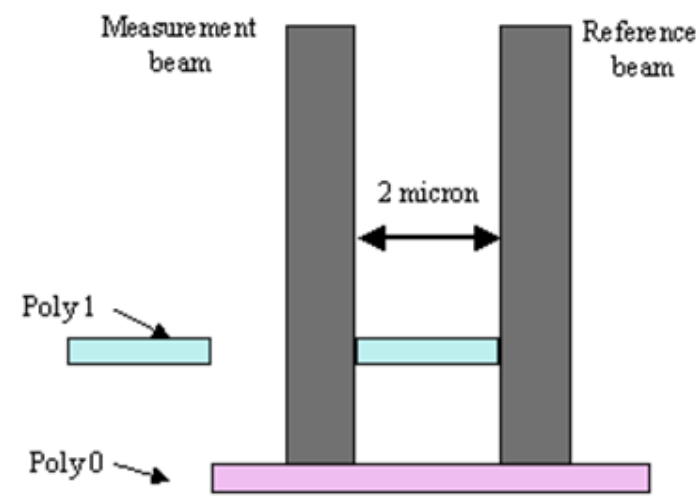

c) Both beams at the same level.

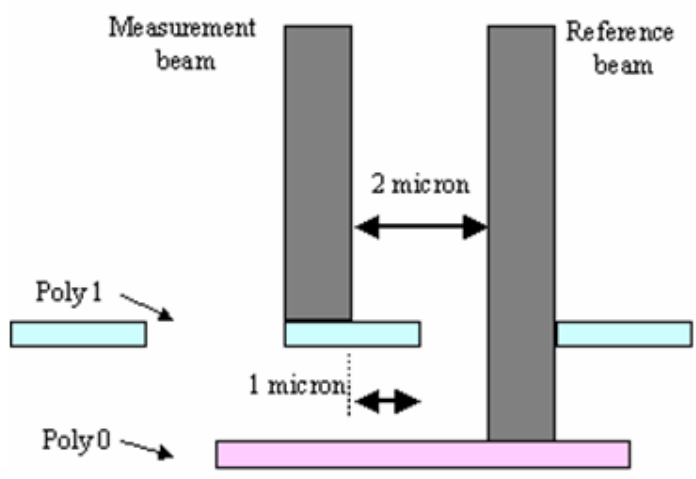

d) 2-micron displacement.

Figure 6.3.1.1, Multi beam displacement measurement system. 


\section{Appendix A: (Control Systems)}

\section{A.1 Some terms and notations}

Some terms and notations are illustrated in details for interested readers to help better understanding of the control approach in this research, however comprehensive control systems can be found in the classical textbooks.

\section{A.2 Positive Definite Matrix}

A scalar $u$, or a function of time $u(t)$, is said to be positive if $u \geq O$ or $u(t) \geq O$ for all $t$. It is strictly positive if $u>O$ or, for some $\alpha>O, u(t) \geq \alpha$ for all $t$.a square matrix $A \in R^{n \times n}$ is positive semi-definite if $x^{T} A x \geq 0$ for all $x$. It is positive definite if , for some $\alpha>0, x^{T} A x \geq \alpha x^{T} x=\alpha|x|^{2}$ for all $x$. Eigenvalues of a positive semi-definite matrix lie in the closed Right Half Plane (RHP), and those of the positive definite lie in the open RHP. If $A \geq 0$ and $A=A^{T}$, then $A$ is symmetric positive semi-definite. The eigenvalues of a symmetric matrix are all real.

\section{A.3 Stability of dynamic system}

Systems are defined as the following differential equation:

$$
\dot{x}=f(t, x) \quad x\left(t_{0}\right)=x_{0}
$$

where $x \in \mathfrak{R}^{n}, t \geq 0$.

The system (A.2.1) is said to be autonomous, or time invariant, if function $f$ does not depend on $t$ and non-autonomous or time varying otherwise. In a closed region like $B$ in $\Re^{n}$ properties are called as the following if:

- Locally, if function is true for all initial conditions in the region $B$.

- Globally, if function is true for $x_{0} \in \mathfrak{R}^{n}$.

- Uniformly, if true for all $t_{0} \geq 0$.

Then the system (A.3.1) is called stable at any equilibrium point if the trajectory $x(t)$ remains close to $\mathrm{o}$ if the initial condition $x_{0}$ is close to the origin.

\section{Lyapunov Theory of Stability}

The equilibrium point is locally stable if, for all $t_{0} \geq 0$ and $\varepsilon>0$, there exists $\delta\left(t_{0}, \varepsilon\right)$ such that

$$
\left|x_{0}\right|<\delta\left(t_{0}, \varepsilon\right) \Rightarrow|x(t)|<\varepsilon \quad \text { For all } t \geq t_{0}
$$


The equilibrium point is globally stable if it is asymptotically stable and $\underset{t \rightarrow \infty}{\operatorname{Lim}}|x(t)|=0$ for all $x_{0} \in \Re^{n}$. where equilibrium point is defined as any point which satisfies $f(t, x)=0$ for all $t \geq 0$.

\section{A.4 Uniform stability}

If in the stability in the sense of Liapunov $\delta$ can be chosen to be independent of $t_{0}$ then the equilibrium point is uniformly stable.

\section{A.5 Asymptotically Stability}

An equilibrium point is asymptotically stable if:

- That is a stable equilibrium point

- That is attractive meaning:

For all $t_{0} \geq 0$ there exist $\delta\left(t_{0}\right)$ such that

$$
\left|x_{0}\right|<\delta \Rightarrow \lim _{t \rightarrow \infty}|x(t)|=0
$$

\section{A.6 Uniform Asymptotic stability}

An equilibrium point is uniformly asymptotically stable if:

- That is uniformly stable point

- Trajectory $x(t)$ converges to o uniformly in $t_{0}$.

\section{A.7 Global Asymptotic Stability}

An equilibrium point is called globally asymptotically stable if that is asymptotically stable and $\lim _{t \rightarrow \infty}|x(t)|=0$ for all $x_{0} \in \mathfrak{R}^{n}$.

\section{A.8 Exponentially Stability, Rate of Convergence}

An equilibrium point is exponentially stable if, there exist $m, \alpha>0$ such that the solution $x(t)$ holds the following equation

$$
|x(t)| \leq m e^{-\alpha\left(t-t_{0}\right)}\left|x_{0}\right|
$$

For all $x_{0} \in B, t \geq t_{0} \geq 0$, the constant $\alpha$ is rate of convergence.

\section{A.9 Persistency Excitation (PE)}

A vector of signals $(w)$ is called persistently exciting, if there exist $\alpha_{1}, \alpha_{2}, \delta>0$ such that: 


$$
\alpha_{2} I \geq \int_{t_{\circ}}^{t_{\circ}+\delta} w(\tau) w^{T}(\tau) d \tau \geq \alpha_{1} I
$$

The $\mathrm{PE}$ conditions require that $w$ has enough rotation in space such that the integral of $w(\tau) w^{T}(\tau)$ becomes uniformly positive definite over any value of $\delta$.

\section{A.10 Function definitions}

A polynomial in Laplace representation is called monic if the highest power in $s$ has coefficient 1 , and is Hurwitz if all of its roots lie in the RHP. A rational transfer function is stable if its denominator polynomial is Hurwitz and it is called minimum phase if its numerator polynomial is Hurwitz. The relative degree of a transfer function is the deference degree between its numerator and denominator; if the relative degree is at least o then the transfer function is called proper and it is strictly proper if its relative degree is at least one.

\section{A.11 Positive Real (PR) and Strictly Positive Real (SPR)}

A transfer function $\hat{M}(s)$ is Positive Real if $\hat{M}(\sigma) \in \mathfrak{R}$ for all $\sigma \in \mathfrak{R}$ and $\operatorname{Re}(\hat{M}(\sigma+j \omega)) \geq 0$ for all $\sigma>0, \omega \geq 0$. The transfer function is Strictly Positive Real if, for some $\varepsilon>0, \hat{M}(s-\varepsilon)$ is PR. In other word, necessary conditions for a transfer function to be SPR is:

- $\hat{M}(s)$ is relative degree 0 or 1

- $\hat{M}(s)$ is minimum phase

- $\hat{M}(s)$ is strictly proper

- The Nyquist plot of $\hat{M}(s)$ lies completely in RHP

The main difference between PR and SPR systems is that a PR system may tolerate poles on the imaginary axis while SPR functions cannot. Some characteristics of the SPR systems are:

- If $\hat{M}(s)$ is SPR, so is $\frac{1}{\hat{M}(s)}$

- If $\hat{M}_{1}(s)$ and $\hat{M}_{2}(s)$ are SPR, so is

$$
\hat{M}(s)=\alpha_{1} \hat{M}_{1}(s)+\alpha_{2} \hat{M}_{2}(s), \text { If } \alpha_{1}, \alpha_{2} \geq 0
$$

- $\quad$ if $\hat{M}_{1}(s)$ and $\hat{M}_{2}(s)$ are SPR, so is

$$
\hat{M}(s)=\frac{\hat{M}_{1}(s)}{1+\hat{M}_{1}(s) \hat{M}_{2}(s)}
$$




\section{Appendix B:}

\section{B.1 MIT Rule}

The first adaptation rule was designed by Dr. Whitaker et al. at Massachusetts Institute of Technology (Parks. 1966) (Osburn, Whitaker, Kezer). Considering the first order system Figure B.1.1., the controller gain adaptation technique is based on minimizing of $\int e^{2} d t$, as

$$
k_{c}=-B e\left(\frac{\partial e}{\partial k_{c}}\right),
$$

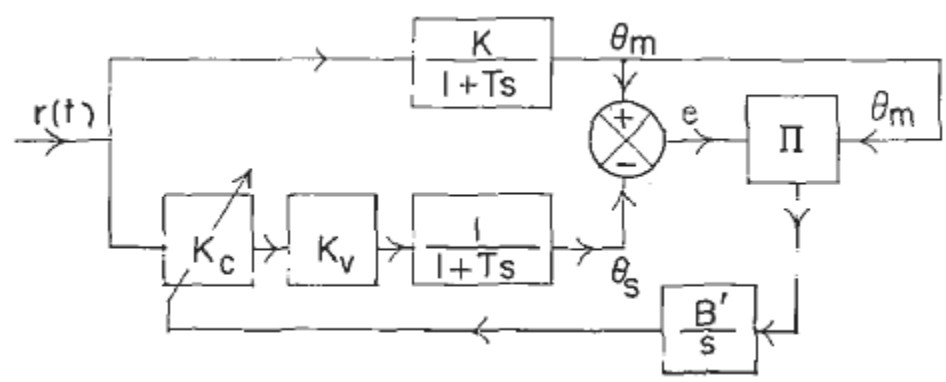

Figure B.1.1. MIT first order system, (Park. 1966).

where $B$, is a positive constant, and

$$
\left(\frac{\partial e}{\partial k_{c}}\right)=\frac{-k_{v} r}{1+T s}
$$

which is found from derivative of the following transfer function with respect to $k_{c}$

$$
e=\frac{\left(k-k_{c} k_{v}\right) r}{1+T s} .
$$

The signal $\frac{\partial e}{\partial k_{c}}$ is generated form additional circuits and $\dot{k}_{c}=B e \theta_{m}$,

where $\theta_{m}$ is given by $\theta_{m}=k R\left(1-\exp \left(-\frac{t}{\tau}\right)\right)$ and error signal holds

$$
T \ddot{e}+\dot{e}+k k_{v} R^{2} B^{\prime} e\left(1-\exp \left(-\frac{t}{\tau}\right)\right)=0 .
$$


As $t \rightarrow \infty$ the equation is equal to $T \ddot{e}+\dot{e}+k k_{v} R^{2} B^{\prime} e=0$ which is asymptotically stable, therefore as time increases the error reaches zero $e \rightarrow 0$ and $k_{c} \rightarrow \frac{k}{k_{v}}$. The system of Figure B.1.1. can be written in space state format as following equations as

$$
\left[\begin{array}{c}
\dot{x} \\
\dot{e}
\end{array}\right]=\left[\begin{array}{cc}
0 & -k_{v} B^{\prime} \theta_{m}(t) \\
\frac{r(t)}{T} & -\frac{1}{T}
\end{array}\right]\left[\begin{array}{l}
x \\
e
\end{array}\right] .
$$

For a simple system, MIT rule leads to a very complicated set of equation, which is hard to solve, and in certain conditions for second order systems, instability is possible. Figure B.1.2 shows the MIT adaptation rule for second order systems.

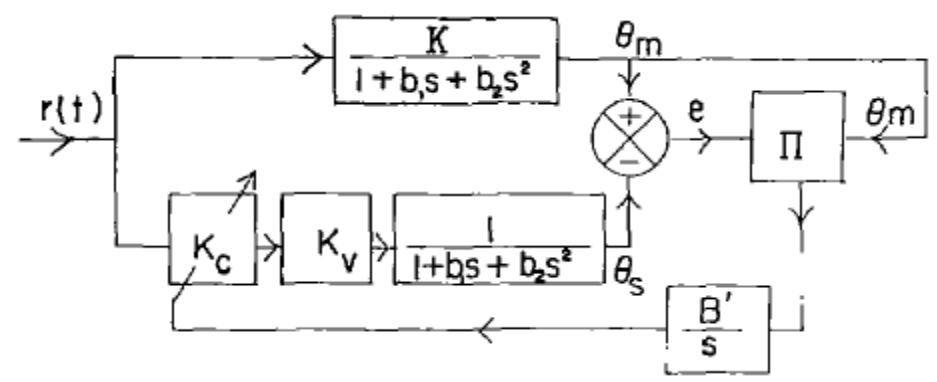

Figure B.1.2. Second order system MIT rule, (Park. 1966).

\section{B.2 The Liapunov Scheme (Parks. 1966)}

Again consider the system of Figure B.1.1, and consider its adaptive step response meaning that the initial value of the gain $\left(k_{v} k_{c}\right)$ is different from $k$. Using a tentative Liapunov function as (Butchart, Shackcloth. 1965)

$$
V=e^{2}+\lambda x^{2}
$$

where $\lambda>0$ is a positive constant, and $x=k-k_{v} k_{c}, \dot{x}=-k_{v} \dot{k}_{c}$. Taking the time derivative of (B.2.1) results in

$$
\frac{d V}{d t}=2 e \dot{e}+2 \lambda x \dot{x}=2 e\left(-\frac{e}{T}+\frac{x r(t)}{T}\right)+2 \lambda x \dot{x}
$$


Making $\dot{x}=\frac{-e r(t)}{\lambda T}$, result in a negative $\frac{d V}{d t}=-\frac{2 e^{2}}{T}<0$ and makes the system asymptotically stable, and $B^{\prime}=\frac{1}{\lambda T k_{v}}$. The asymptotically stability of this method is guaranteed if $r(t) \neq 0$.Therefore the error dynamics of the system becomes

$$
T \ddot{e}+\dot{e}+k_{v} R^{2} B^{\prime} e=0,
$$

with initial conditions $e=0, \dot{e}=\frac{1}{T}\left(k-k_{v} k_{c}\right) R$ at $t>0$ with $r(t)=R$ being a constant input. The adaptation technique results in the following rule

$$
\dot{k}_{c}=e r B^{\prime} .
$$

Figure B.2.1 shows the adaptation technique and the redesign of the MIT rule for first order systems.

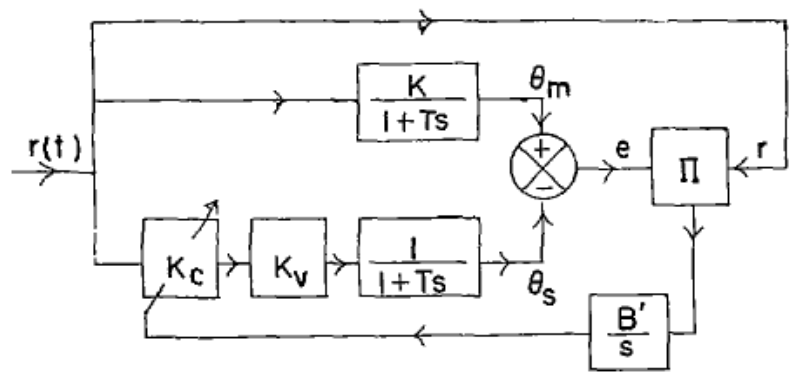

Figure B.2.1, Liapunov design for first order systems, (Park. 1966).

For second order systems the Liapunov function will be formed as a quadratic $Q$ in $e$ and $\dot{e}$ for the second order equation, as shown in Figure B.2.2, $b_{2} \ddot{e}+b_{1} \dot{e}+e=0$. One quadratic form of $Q$ is based on (Parks. 1964) is

$$
\begin{gathered}
Q=\frac{b_{1}}{b_{2}^{2}} e^{2}+\frac{b_{1}}{b_{2}} e^{2} \\
\dot{Q}=-2\left(\frac{b_{1}}{b_{2}}\right)^{2} \dot{e}^{2}
\end{gathered}
$$

Thus a suitable Liapunov function can be 


$$
V=\frac{b_{1}}{b_{2}^{2}} e^{2}+\frac{b_{1}}{b_{2}} \dot{e}^{2}+\lambda x^{2}
$$

where $x=k-k_{v} k_{c}$, yeilds

$$
\frac{d V}{d t}=-2\left(\frac{b_{1}}{b_{2}}\right) \dot{e}^{2}+2 \frac{b_{1}}{b_{2}} \dot{e} x r(t)+2 \lambda x \dot{x} .
$$

Choosing $\dot{x}=-k_{v} \dot{k}_{c}=\frac{-b_{1} e r}{\lambda b_{2}}$, the adaptation rule is

$$
\dot{k}_{c}=B^{\prime} \dot{e} r
$$

where $B^{\prime}=\frac{b_{1}}{\lambda b_{2} k_{v}}$

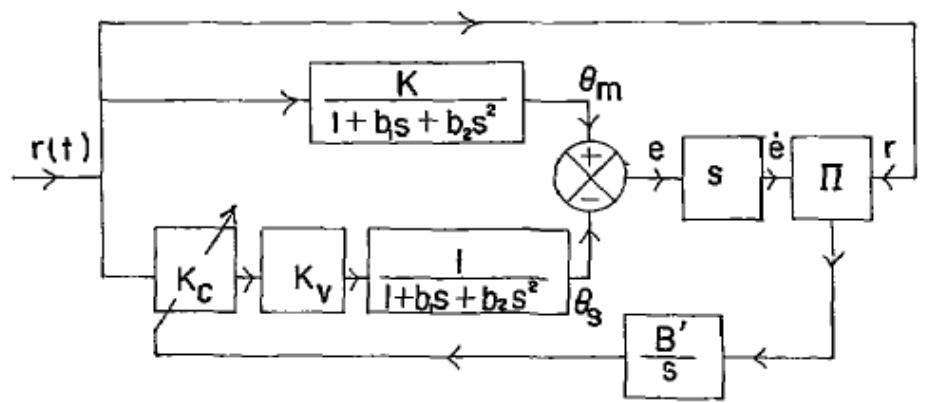

Figure B.2.2. Liapunov design for second order systems, (Park. 1966).

Figure B.2.2 shows the Liapunov design for second order systems. As it is seen the adaptation loop makes use of the derivative of the error signal, which is the weak point of this algorithm and has been criticized in (Butchart, Shackcloth. 1965).

\section{B.3 Dressler Design Rule (Dressler. 1967)}

In this adaptation technique the parameter adjustment is based on

$$
\dot{k}_{c}=B^{\prime} e_{1} r
$$

where $e_{1}$ is the tracking error. Figure B.3.1 shows the Dressler method for adaptive control gain adjustment. The resulting controller has a very simple structure. The disadvantages of this 
method are the high damping rate of the response and the lack of stability proof which is the same as MIT rule (Hang, Parks. 1973).

\section{B.4 Price Design Rule (Price. 1970)}

The accelerated gradient method with constant $\gamma_{c}$, has a parameter adjustment rule such as

$$
\dot{k}_{c}=B^{\prime} e_{1} r+\gamma_{c} \frac{d}{d t}\left(B^{\prime} e_{1} r\right)
$$

The controller has the same structure as Dressler with an additional part of the feed-forward term. This term improves the stability of the controller as well as the damping response of the system. In higher order systems, the stability of the system is impaired. Figure B.3.1 shows the price method (Hang, Parks. 1973).

\section{B.5 Monopoli Design Rule (Monopoli, Gilbert, Thayer. 1968)}

Based on the Liapunov modification a differentiating block $z(s)$ is used to modify the plants transfer function in order to $z(s) \frac{N(s)}{D(s)}$ is positive real, after eliminating the error derivatives using Kalman-Meyer Lemma the adaptation technique is written as

$$
\dot{k}_{c}=B^{\prime} e_{\mid} y+\gamma \frac{d}{d t}\left(B^{\prime} e_{\mid} y\right),
$$

where $y$ is the modified input signal to the plant and can be obtained by multiplying the original signal by $\frac{1}{z(s)}$. For an $n^{\text {th }}$ order system with $m$ zeros the polynomial $z(s)$ is the order of $(n-m-1)$. Since there are $(n-m-1)$ number of derivatives, the system is stable (Monopoli, Gilbert, Thayer. 1968). This system is suitable for time-varying gain (Hang, Parks. 1973). Figure B.3.1 shows this method. 

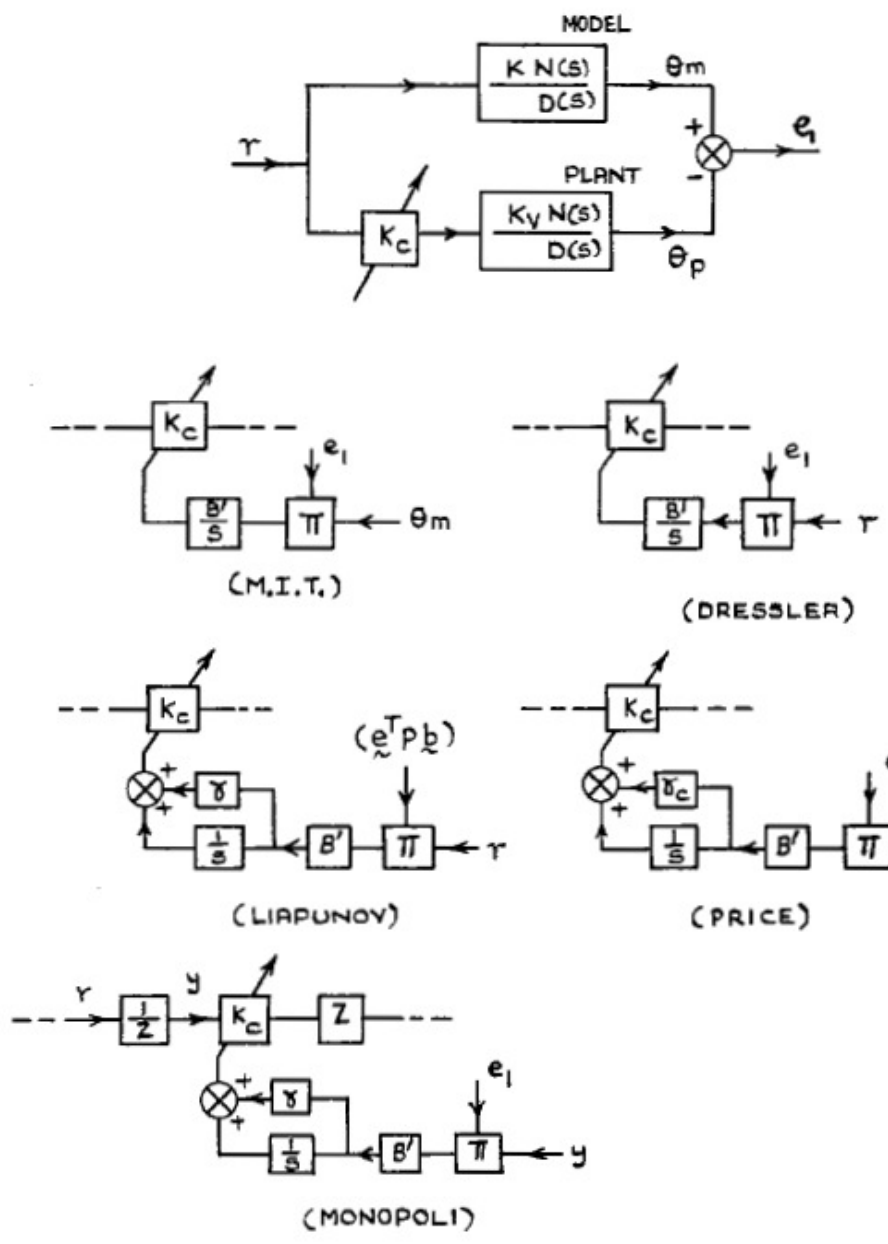

Figure B.3.1. Designs of the adaptive loops (Hang, Parks. 1973) 


\section{References}

Astrom, K. J. and Wittenmark, B. (1995). A Survey of Adaptive Control Applications, in Proc. Conf. on Decision and Control, December pp. 649-654.

Bertran, E. and G.M, Lopez. (1995), Adaptive VS-MRAC for disturbance cancellation. Electronics Letters, 19th January, vol. 31, no. 2, pp 142- 144 .

Bellman, R. E. (1961). Adaptive Control Processes. Princeton University press. Princeton NJ.

Bhansali, S. Zhang, A. L. Zmood, R. B. Jones, P. E. and Sood, D. K. (2000). Prototype Feedback-controlled bi-directional actuation system for MEMS applications. Journal of Microelectromechanical systems, v 9, no 2, June, 2000, pp. 245251.

Blanke, M. Kinnaert, M. Lunze, J. Staroswiecki, M. Schröder, J. (2006). Diagnosis and Fault-Tolerant Control. Springer; Second edition. Chapter 1.

Bryzek, Abbot, J. E. (2003). Control issues for MEMS. 42nd Proceedings of the IEEE Conference on Decision and Control, Maui, Hawaii, USA, December 2003.

Brown, S. B. and Jansen, E. (—). Reliability and Long Term Stability of MEMS. WB1 invited paper, — —pp.9-10.

Butchart, R. L. \& Shackcloth, B. (1996). Synthesis of model reference adaptive control systems by Liapunov second method. Proc.1965 IFAC Symp. On Adaptive Control (Teddington, England) Instrument Society America, 1996.

Campbell, S. L. and Nikoukhah, R. (2004). Auxiliary Signal Design for Failure Detection. Princeton University Press.

Chan, Y. T. (1973). Perfect model following with a real model. Proc. Joint Automat. Contr. Conf. 1973, pp. 287- 293.

Chueung, P. Horowitz, R. and Howe, R. T. (1995). Identification, Position sensing and control of an Electrostatically Driven Polysilicon Micro actuator. Proceeding of the 34th Conference on Decision and Control, New Orleans, LADecember 1995.

Chien, C. - J. \&. Fu, L. C (-). Adaptive control systems. Chapter 3, pp 41-57.

Dawson, J. M. Chen, J. Brown, K. S. Famouri, P. Hornak, L. A. (2000). Through wafer optical probe characterization for micro electromechanically systems positional state monitoring and feedback control. Optical engineering, Vol. 39, No. 12, Pp 3239-3246.

Dawson, J. M. (2002). Integrated through Wafer Optical Monitoring of MEMS for closed loop Control. PhD dissertation West Virginia University WVU Morgantown, fall 2002.

Dawson, J. Wang, L. Chen, J. Famouri, P. and Hornak, L. A. (2000). MEMS Feed back Control Using through -Wafer Optical Device Monitoring. Proceeding of SPIE, The international Society for Optical Engineering Volume 1478. 18-20 September 2000, Santa Clara, USA.

Dellman, J. Akiyama, T. D. Briand, Gautsch, S. Guenat, O. Guldimann, B. Luginbuhl, P. Marxer, C. Staufer, U. Van DerSchoot, B. and de Rooij, N. F.(200o). Microsystems for diverse applications using recently developed micro fabrication techniques. Proc. SPIE MOEMS and Miniaturized systems, Vol. 4178, pp. 16-27, Sept. 2000.

Dressler, R. M.(1967).An approach to model reference adaptive control systems. IEEE Trans. Automat. Contr., vol. AC-12, pp 75-80, Feb. 1967.

Erzerberger, H. (1968). Analysis and design of model following systems by state space techniques. In Proc. Joint Automat. Contr. Conf. 1968, pp. 572-581.

Fedder, G. K. and Howe, R. T (1997). Multimode Digital Control of Suspended Polysilicon Microstructure. IEEE, Journal of Microelectromechanical systems, vol. 5, no. 4, December. pp. 283-297.

Fedder, G. K. (1996). Issues in MEMS Micro modeling. IEEE, 2003, o-7803-8135-1, pp. 64-69.

Friedland, B. (1996). Advanced Nonlinear Control, Prentice-Hall.

Fiedziuszko, S. J. (-). Application of MEMS in Communications Satellites. IEEE. Page 201-211.

Furuta, K. Kosuge \& K. Kobayashi, K. (1989). VSS-type Self-Tuning Control of Direct-Drive Motor,” IEEE 1989 page 281286.

Furuta, K. (1991). VSS type Self-Tuning controller. IECON'91. pp 2085-2089.

Gambier, A. (2004). Multivariable Adaptive State-space Control: A Survey. in Proc. 5th Asian Control Conference, 2004, pp. 185-191. 
Garcia, et al. (1995). Surface micro machined micro engine. Sensors and Actuators, Vol. 48 (3) pp. 203- 214, 1995.

Gad-el-Hak, M. (2001). The MEMS Handbook, CRC Press.

Gyimesi, M. Avdeev, I. \& Ostergaard, D. (2004). Finite- Element simulation of Micro-Electromechanical systems (MEMS) by Strongly Coupled Electromechanical Transducers. IEEE Transaction on Magnetics, vol. 40, no. 2, March 2004.

Hanlon, P. Maybeck, P. S. (2000). Multiple-Model Adaptive Estimation Using a Residual Correlation Kalman Filter Bank. IEEE, Transaction on Aerospace and Electronic Systems, Vol. 36, No. 2, April, pp 393-406.

Hang, C. C \& Parks, P. C. (1973). Comparative Studies of Model Reference Adaptive Control Systems. IEEE Transaction on Automatic Control, Vol. AC-18, pp. 419-428, Oct. 1973.

Horowitz, R. Cheung, P. Howe, R. T (-). Position sensing and control of electrostatically driven polysilicon Micro actuator. $-\mathrm{P}-1, \mathrm{P}-2$.

Hsu, L. (1988). Variable Structure Model reference Adaptive Control (VS-MRAC) Using only input and Output Measurements: Part II. Decision and Control Conference, Austin Texas, December 1988, pp 2396- 2401.

Hsu, Y. Y. \& Cheng, C. H. (1998). Variable Structure and Adaptive Control of a Synchronous Generator. IEEE Transaction on Aerospace and Electronic

Hung, J. Y. Gao, W. Hung, J. C.(1993). Variable Structure Control: A Survey. IEEE Trans. On Industrial Electronics, Vol. 40, no. 1, February 1993. pp 2-22.

Hus, L. Lizarralde, F. (1996). Experimental Results on Variable Structure Adaptive Robot control without joint Velocity Measurement. Proceeding of the American Control Conference, Seattle, Washington. June 1996. Pp 2317- 2321.

Hung, J. Y. Gao, W. Hung, J. C. (1993).Variable Structure Control: A Survey. IEEE Transaction on Industrial Electronic, Vol. 40, No. 1, February 1993.

Ikuta, K. Nokota, M. \& Aritomi, S. (1994). Biomedical Micro Robots Driven by Miniature Cybernetic Actuator. 0-78031833-1/94, IEEE 1994, pp. 263-268

Itkis, Y. (1976). Control Systems of Variable Structure. New York: Wiley 1976.

Izadian, A. Hornak, L. and Famouri, P. (2006). Adaptive Control of MEMS Devices. The International Conference on Intelligent Systems and Control, ISC 2006, August 14-16, 2006 Honolulu, Hawaii, USA.

Izadian, A. Wang, L. Dawson, J. M. Hornak, L. A. \& Famouri. P. Closed-Loop Optical Feedback Trajectory Control of Lateral Comb Resonators. Submitted to Control System Technology (2007)

Izadian, A. Famouri, P. (2008). Intelligent Fault Diagnosis of MEMS Lateral Comb Resonators Using Multiple Model Adaptive Estimators. Submitted to IEEE Transaction on Control System Technology.

Izadian, A. Hornak, L. A. Famouri, P. (2007). Trajectory Control of MEMS Lateral Comb Resonators under Faulty Conditions. American Control Conference, 11-13 July, 2007. New York, USA.

Izadian, A. Famouri, P. (2008). Reliability Enhancement of Lateral Comb Resonators under Fault Conditions. IEEE Transaction of Control System Technology.

Izadian, A, Hornak, L. Famouri, P. (2008). Structure rotation and Pull-in Voltage Control of Lateral Micro Comb Resonators. In Press IEEE Transaction on Control System Technology (2008).

Izadian, A. Khayyer, P. Famouri, P. (2008). Fault Diagnosis of Time-Varying Parameter Systems with Application in MEMS LCRS's. Transaction on Industrial Electronic, Invited Paper, 2008.

Ioannou, P. and Fidan B. (2006). Adaptive Control Tutorial. (Philadelphia, Siam).

Judy, J. W. (2001). Microelectromechanical systems (MEMS): fabrication, design and applications. Smart material and Structures, Institute of physics publishing, 2001, [online]: http://www.stacks.iop.org/SMS/10/1115.

Jiang, X. Bhave, S. A. Seeger, J. L. Have, R. T. \& Boser, B. E. (2002). $\Sigma \Delta$ Capacitive interface for vertically driven X \& Y Axis rate Gyroscope. ESSCTRC 2002. pp. 639-642.

Keung. K. \& Young, D. (1978). Design of Variable Structure Model Following Control Systems. IEEE Transaction on Automatic Control, Vol. AC- 23, No. 6, December 1978.

Khamesse, M. B. Kato, N. and Nakamaura, T. (2003). Performance improvement of a magnetically levitated micro robot using an adaptive control. Proceeding of the International Conference on MEMS, NANO and smart Systems (ICMENS'O3) 2003 IEEE. 
Kolpekwar, A. and Blanton, R. D. (1997). Development of MEMS testing Methodology. IEEE International Test Conference, pp. 923-931.1997

Kolpekwar, A. Kellen, C. and Blanton, R. D. (1999). MEMS Fault Model Generation using CARAMEL. Journal of Microelectromechanical Systems, Vol. 8, no. 3, September 1999. pp. 308-318.

Kolpekwar, A. and Blanton, R. D. (1997). Development of MEMS testing Methodology. IEEE International Test Conference, pp. 923-931.1997.

Konduparthi, D. L. (2005). Optical Detection of Multiple Faults on MEMS based Linear Comb Resonator, Master's Thesis, West Virginia University 2005, and [On-line Abstract]. Available: https://eidr.wvu.edu/etd/documentdata.eTD?documentid=4424

Kuipers, B. Astrom, K. (1994). The composition and validation of heterogeneous control laws. Automatica 30, pp 233-249

Levine, W. S. (1996). The Control Hand Book, CRC Press, IEEE Press. pp 941Monopoli, R. V. (1974). model reference applied control with an augmented error signal. IEEE Trans. on Automat. Contr. Vol. AC-19-pp 474-484 Oct. 1974.

Leland, R. P. (2006). Adaptive Control of a MEMS Gyroscope Using Lyapunov Methods. IEEE Transactions on Control Systems Technology, Vol. 14, No. 2, p. 278 - 283, March, 2006.

Leus, V. \& Elata, D. (2004). Fringing Field Effect in Electrostatic Actuators. Technical Report ETR-2004-2, MAY 2004, Technicon -Israel Institute of Technology, Faculty of Mechanical engineering.

Li, J. P. and Thompson, G. (2005). A Method to Take Account of Inhomogeneity in Mechanical Component Reliability Calculations. IEEE Transactions on Reliability , vol. 54, no. 1, March 2005, pp. 159-168

Li Y. and Horowitz, R. (2001). Active suspension vibration control with dual stage actuators in hard disk drives. Proceedings of American Control Conference, Arlington, VA June 25-27, pp. 2786-2791.

Liu, Y. E. Skormin, V. A. (1988) Variable Structure Adaptive Control. IECON, 1988, pp 281-286.

Masuda, S. \& Inoue, A. (1995). Variable Structure Model Reference Adaptive Control Based on Two -Degree- of- Freedom Compensators Scheme. American Control Conference, Seattle, Washington, June 1995, pp 3012- 3016.

McCormick, W. (2004). Diffractive Optical Lens Design for Integrated Monitoring of Microelectromechanical Lateral Comb Resonator, Master's Thesis, West Virginia University.

Monopoli, R. (1974). Model reference adaptive control with an augmented error signal. IEEE Trans. On Automatic Control, vol. 19, no. 5, pp. 474-484, October 1974.

Monopoli, R. V. Gilbert, J. W. \& Thayer, W. D. (1986). Model reference adaptive control based on Liapunov-like techniques. Proc. 2nd IFAC Symp. System Sensitivity and Adaptivity (Dubrovnik, Yugoslavia), Aug. 1968, pp. F.24F.36.

Murray Smith, R. and Johansen, T. A. (1997). Multiple Model Approaches to Modeling and Control, Taylor \& Francis.

Opoitsev, V. I. (一). Identification of static plants by means of pieces wise linear functions. Automatic and Remote Control $31, \mathrm{pp} 809-815$.

Osiander, R. Firebaugh, S. L. \& Champion, J. L. Farrar, D. \& Darrin, M. A. G.(2004). Microelectromechanical Devices for Satellite Thermal Control. IEEE Sensors Journal, vol. 4, no. 4, August 2004. pp. 525-531.

Osburn, P. V. Whitaker, H. P. \& Kezer, A. (-). New Development in design of adaptive control systems. Inst. Of Aeronautical Sciences, paper 61-39.

Park, S. Horowitz R. and Tan, C. W. (2001). Adaptive controller design of MEMS gyroscope. IEEE Intelligent Transportation System Conference Proceeding- Oakland (CA), USA- August 25- 29, 2001.

Park, S. and Horowitz, R. (2003). Adaptive Control for the Conventional Mode of Operation of MEMS Gyroscopes. J. Microelectromechanical Systems, Vol. 12, pp. 101-108, Feb. 2003.

Parks, P. C. (1964). A new look at the Ruth-Hurwitz problem using Liapunov second method. Bull. Polish acad. Sci., Tech. Sci. Ser., vol. 12, pp. 19-21, June 1964.

Parks, P. C. (1966). Liapunov redesign of Model Reference Adaptive Control Systems. IEEE Transaction on Automatic Control., vol. AC-11, pp. 362-367, July 1966. 
Pannu, S. Chang, C. Muller, R. S. and Pisano, A. P. (2000). Closed-loop feedback control systems for improved tracking in magnetically actuated micro mirrors. IEEE/LEOS International Conference on Optical MEMS, Aug. 21-24, Kauai, HI, USA, pp 107-108.

Reddy, P. S. \& Jessing, J. R. (2004). Pattern Alignment Effects in Through-Wafer Bulk Micromachining of (100) Silicon. IEEE Conference 2004, 0-7803-8369-9/04.

Price, C. F (1970). An accelerated gradient method for adaptive control. Proc. 9th IEEE Symp. Adaptive Processes Decision and Control, Dec. 1970, pp. IV. 4. 1-4.9.

Powell, M. J. D. (-). Radial Basis Functions for multivariable Interpolation: A review in algorithms for approximation. Clarendon Press. Oxford. Pp. 143-167.

Rogers, J. W. Phinney, L. M. (2001). Process Yield for Laser Repair of Aged, Stiction-Failed, MEMS Devices,” Journal of Microelectromechanical Systems, vol. 10, no. 2, pp. 280-285, June 2001.

Rosing, R. Richardson, A. Dorey A. \& Peyton, A. (1999). Fault Simulation for MEMS. Intelligent and Self-Validating Sensors (Ref. No. 1999/160), Colloquium on IEE. 21 June 1999. pp. 7/1 - 7/6.

Samper, V. and Trigg, A. (2003). MEMS Failure Analysis and Reliability. Proceeding of 10th IPFA, 2003, Singapore, pp. 17-24.

Schanwald, L. P. Schwank, J. R. Sniegowski, J. J. Walsh, D. S. Smith, N. F. Peterson, K. A. Shaneyfelt, M. R. Winokur, P. S. Smith, J. H. and Doyle, B. L. (1998). Radiation Effects on Surface Micro machined Comb Drives and Microengine. IEEE Transaction on Nuclear Science, Vol. 45, no. 6, December 1998. pp. 2789-2798.

Schanwald, L. P.. Schwank, J. R Sniegowski, J. J. Walsh, D. S. Smith, N. F. Peterson, K. A. Shaneyfelt, M. R. Winokur, P. S. Smith, J. H. and Doyle, B. L. (1998). Radiation Effects on Surface Micro machined Comb Drives and Microengine. IEEE Transaction on Nuclear Science, Vol. 45, no. 6, December 1998. pp. 2789-2798.

Senturia, S. D (2001). Micro System Design, KAP press, Ch. 11.

Shams, Q. A. Moniuszko, M. \& Ingham, J. C. (2001). Applying MEMS Technology to Field, Flight \& Space Deployable System. 0-7803-7022-8/10. IEEE 2001, pp. 246-255.

Shkel, A. M. Horowitz, R. H. Seshia, A. A. Park, S. Howe, R. T. (1999). Dynamics and Control of Micromachined Gyroscopes. American Control Conference, San Diego, CA. Hune. pp. 2119-2124.

Slotine, J. J. E. \& Li, W. (1991). Applied Nonlinear Control. Prentice Hall, Inc.

Sniegowski, J. J. and Garcia, E. J. (-). Micro-fabricated actuators and their application to optics. Proc SPIE, Vol. 2384, Pp. 46- 64.

Srikar, V. T. and Senturia S. D. (2002). The Reliability of Microelectromechanical Systems (MEMS) in Shock Environment. Journal of Microelectromechanical Systems, vol. 11, no. 3, June 2002, pp. 206-214.

Stromberg, J. E. Gustafsson, F. Ljung, L. (1991). Trees as black box model structures for dynamical systems. European Control Conference, Grenoble, pp 1175-1180.

Tso, S. K. Xu, Y. Shum, H. Y. (1991). Variable Structure Model Reference Adaptive Control of Robot Manipulators. International Conference on Robotics and Automation, Sacramento CA. April 1991, pp 2148- 2153.

Tang, W. T. Lim, M. G. and Howe R. T. (1992). Electrostatic Comb Drive Levitation and Control Method. IEEE, Journal of Microelectromechanical systems, vol. 1, no. 4. December. pp. 170-178.

Tsuchiya, T. Tabata, O. Sakata, J. and Taga, Y. (1997). Specimen Size Effect on Tensile Strength of Surface Micromachined Polycrystalline Silicon Thin Films. IEEE, 1997, 0-7803-3744-1/97. pp. 529-534.

Utkin, V. I. (1992). Sliding Modes in Control and optimization. Berlin: Springer Verlag.

Utkin V. I. \& Yang, K. D. (1978). Methods for Constructing Discontinuity Planes in Multidimensional Variable Structure Systems," Automation and Remote Control, 39(1978), pp. 1466-1470

Wahba, G. (1990). Spline models for observation data. Regional Conference Series in Applied Mathematics, SIAM. Philadelphia, PA.

Wamg, F. Hong, B. Brandt, S. A. Darrell. \& Long, D. E. (2005). Using MEMS-based Storage to Boost Disk Performance. Proceedings of the 22nd IEEE/ 13th NASA Doddard Conference on Mass Storage Systems and Technologies (MSST'05) 
Wang, L. Dawson, J. M. Chen, J. Famouri, P. and Hornak, L. A. (200o). Stroke-Length control of a MEMS device. ISIE, Cholula, Puebla, Mexico.

Wang, L. (2005). Modeling and Real-Time Feedback Control of MEMS Device, PhD. Dissertation, West Virginia University, 2005. [On-line Abstract]. Available: https://eidr.wvu.edu/etd/documentdata.eTD?documentid=3711

Wang, L. Dawson, J. M. Hornak, L. A. Famouri, P. Ghaffarian, R. (2004). Real Time Transitional control of a MEMS comb resonator. IEEE transaction on Aerospace and electronics. Vol. 40, No. 2 April.

Wellstead, P. E. and Zarrop, M. B. (1991). Self-tuning Systems control and signal processing. John Wiley \& Sons.

Whittaker, J. (1990). Graphical models in applied multivariable statistics. John Wiley \& Sons.

Wu, X. Zhe, J. Wang, J. Cheng, J. Modi, V. \& Farmer, K. R. (-). A Generalized Capacitance-Based model for Electrostatic Micro- Actuators. [online]: www.njit.edu/v2/MRC/mrcpdf/wuappliedphysicsletter_v6.pdf

Xu, J. X. Heng, T. M. Wang, L. (1996). Self-Tuning Type Variable Structure Control Method for a Class of Nonlinear Systems. IEEE 1996 Workshop on Variable Structure Systems.

Young, K. K. D. (1978). Design of Variable Structure Model Following Control Systems. IEEE Transaction on Automatic Control, VOL. AC-23, NO. 6, December 1978.

Zadeh, L. A. (-). Outline of a new approach to the analysis of complex systems and decision processes. IEEE Trans. Systems, Man, Cybernetics 3, 28-44.

Zhang, X. and Tang, W. C. (1994). Viscous air damping in laterally driven micro resonators. IEEE, Micro Electro Mechanical Systems Conference, 1994, pp. 199 - 205.

Zhuang, W. (1999). RLS Algorithm with Variable Forgetting Factor for Decision Feedback Equalizer over Time-Variant Fading Channels. http://www.cwc.uwaterloo.ca/tech_reports/zhuang3.pdf 